\title{
t \\ Robotics Deep Reinforcement Learning with Loose Prior Knowledge
}

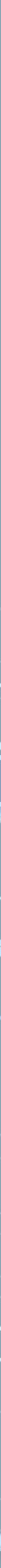


ROBOTICS DEEP REINFORCEMENT LEARNING WITH LOOSE PRIOR KNOWLEDGE

Nicolò Botteghi 



\title{
ROBOTICS DEEP REINFORCEMENT LEARNING WITH LOOSE PRIOR KNOWLEDGE
}

\author{
DISSERTATION
}

to obtain

the degree of doctor at University of Twente,

on the authority of the rector magnificus,

prof. dr. ir. A. Veldkamp,

on account of the decision of the Doctorate Board,

to be publicly defended

on Wednesday 6 October 2021 at 16.45 hours

by

\section{Nicolò Botteghi}

Born on the $10^{\text {th }}$ of November, 1992

in Bologna, Italy 
This dissertation has been approved by:

\author{
Supervisors \\ prof. dr. ir. S. Stramigioli \\ prof. dr. ir. C. Brune \\ Co-Supervisors \\ dr. M. Poel
}

Cover design: Daan Geijs and Nicolò Botteghi

Printed by: Ipskamp Printing

Lay-out: Daan Geijs and Nicolò Botteghi

ISBN: $\quad$ 978-90-365-5216-5

DOI: $\quad 10.3990 / 1.9789036552165$

(C)2021 Nicolò Botteghi, The Netherlands. All rights reserved. No parts of this thesis may be reproduced, stored in a retrieval system or transmitted in any form or by any means without permission of the author. Alle rechten voorbehouden. Niets uit deze uitgave mag worden vermenigvuldigd, in enige vorm of op enige wijze, zonder voorafgaande schriftelijke toestemming van de auteur. 


\section{Graduation Committee:}

Chair

Supervisors

Co-supervisor

Members prof. dr. J.N. Kok

prof. dr. ir. S. Stramigioli prof. dr. ir. C. Brune

dr. M. Poel

prof. dr. D.K.J. Heylen dr. ir. L.J. Spreeuwers prof. dr. H.H. Hoos

dr. ing. J. Kober prof. dr. ir. R.N.J. Veldhuis
University of Twente

University of Twente University of Twente

University of Twente

University of Twente University of Twente Leiden University TU Delft

University of Twente

The research presented in this thesis has been carried out at the Robotics and Mechatronics (RaM) group in the Department of Electrical Engineering, Mathematics and Computer Science (EEMCS) at the University of Twente. This work has been financially supported by the Smart Tooling project, an Interreg Flanders-Netherlands project sponsored by the European Union (https://smarttooling.eu/). 

Two roads diverged in a wood, and I-I took the one less traveled by, and that has made all the difference. Robert Frost - The Road Not Taken 



\section{Summary}

Robotics research has tremendously progressed in the last decade, and robots can now be programmed and automated for solving different tasks: from everyday life simplest jobs, e.g. vacuum cleaning, or grass-cutting, to complex industrial applications, e.g. car assembly, smart warehouse, or inspection of plants. However, many steps have yet to be taken to achieve high degrees of cognitive and motoric intelligence and autonomy. These automated solutions require a vast amount of prior knowledge of the tasks and are often brittle in all the scenarios in which the robots have an imperfect and limited perception, inaccurate models of the world and uncertainties in the motion.

Learning from interaction is the simplest yet strongest learning approach that every living being experiences throughout its life. Most of what humans and animals learn is built on the concept of iteratively acting and improving the behaviour based on the consequences of the actions taken. When designing artificial brains for autonomous robots, what inspires us is the idea of simply letting the robot's brain autonomously learn how to act, i.e. control the robot, in the best way for solving a given task. Unfortunately, artificial brains do not learn as fast as living beings' brains, and even solving simple problems requires thousands or millions of unsuccessful trials.

In this thesis, we focus on the study of the methods that tackle the perceivingreasoning-acting chain with the general learning approach of learning from interaction for solving different robotics tasks, such as navigation, path planning and exploration. This paradigm of learning is commonly known as Reinforcement Learning [1]. In particular, we study how representations of the information perceived by the robot and of the decisions of the artificial mind can be learned and how these representations can be used to simplify reasoning and acting in Reinforcement Learning. Moreover, we study how to reward the artificial brain to 
viii

achieve better behaviours and how this translates to different robotics tasks.

We show that loose forms of prior knowledge are the ones to employ in robotics because they do not limit nor constrain the search of the optimal behaviour but instead improve the agent's ability to adapt to different and unseen-a-priori situations, i.e. the generalisation, the ability to learn fast with limited experience, i.e. the sample efficiency, and the ability to behave well in the presence of uncertainties, i.e. the robustness of the learned behaviours. 


\section{Samenvatting}

Robotica-onderzoek is het afgelopen decennium enorm vooruitgegaan en robots kunnen nu worden geprogrammeerd en geautomatiseerd voor het oplossen van verschillende taken, variërend van de eenvoudigste taken van het dagelijks leven (stofzuigen of grasmaaien) tot complexe industriële toepassingen (automontage, slim magazijnbeheer of inspectie van planten). Er moeten echter nog veel stappen worden gezet om een hoge mate van cognitieve en motorische intelligentie en autonomie te bereiken. Deze geautomatiseerde oplossingen vereisen een enorme hoeveelheid voorkennis van de taken en zijn vaak broos in alle scenario's waarin de robots een onvolmaakte en beperkte perceptie hebben (1), onnauwkeurige modellen van de wereld hebben (2) en omgaan met onzekerheden in de beweging (3).

Leren door interactie is de eenvoudigste maar sterkste leerbenadering die elk levend wezen gedurende zijn hele leven ervaart. Het meeste van wat mensen en dieren leren, is gebaseerd op het concept van iteratief handelen en het verbeteren van het gedrag op basis van de gevolgen van de ondernomen acties. Bij het ontwerpen van kunstmatige hersenen voor autonome robots is het inspirerend om op dezelfde manier te werk te gaan en eenvoudigweg het brein van de robot autonoom te laten leren hoe die de robot het beste kan laten gedragen, d.w.z. de robot te besturen, om een bepaalde taak op te lossen. Helaas leren kunstmatige hersenen niet zo snel als de hersenen van levende wezens, en zelfs het oplossen van eenvoudige problemen vereist duizenden of miljoenen pogingen.

In dit proefschrift richten we ons op de studie van de methoden die de waarnemenredeneren-doen keten behandelen gebaseerd op de algemene leerbenadering van leren door interactie voor het oplossen van verschillende robottaken, zoals navigatie, pad planning en verkenning. Dit paradigma van leren staat algemeen bekend als Reinforcement Learning [1]. In het bijzonder bestuderen we hoe representaties van de waargenomen informatie van de wereld en de beslissingen van de agent 
kunnen worden geleerd en hoe deze representaties kunnen worden gebruikt om redeneren en handelen te vereenvoudigen bij Reinforcement Learning. Bovendien bestuderen we hoe we de handelende entiteit kunnen belonen om beter gedrag te bereiken en hoe dit zich vertaalt naar verschillende robottaken.

We laten vormen van voorkennis zien die niet alleen het zoeken naar het optimale gedrag niet begrenzen of beperken, maar daarnaast ook het vermogen van de agent verbeteren om zich aan te passen aan verschillende en ongeziene a-priori situaties (de generalisatie) (1), het vermogen om snel te leren met beperkte ervaring (steekproefefficiëntie) (2), en het vermogen om zich goed te gedragen in aanwezigheid van onzekerheden (de robuustheid van het geleerde gedrag) (3) zijn degenen die in robotica moeten worden gebruikt. 


\section{Sommario}

La ricerca in robotica è tremendamente progredita nell'ultimo decennio e i robot possono ora essere programmati per risolvere diversi compiti: dal più semplice lavoro della vita quotidiana, per esempio la pulizia di pavimenti o il taglio dell'erba, fino a complesse applicazioni industriali come assemblaggio di autovetture o la ispezione di impianti. Tuttavia, molti progressi rimangono necessari per ottenere alti livelli di intelligenza cognitiva, motoria e di autonomia. Queste soluzioni automatizzate richiedono un enorme quantitativo di conoscenze pregresse del compito da svolgere e sono spesso non ottimali in tutti gli scenari in cui il robot possiede percezione imperfetta e limitata, modelli del mondo inaccurati e incertezze nel movimento.

Apprendere tramite interazione è la forma più semplice e forte di apprendimento che ogni essere vivente utilizza durante la propria vita. Molto di ciò che esseri umani e animali apprendono è basato sul concetto di agire e migliorare il comportamento sulla base delle conseguenze della decisione presa. Quando costruiamo menti artificiali per robot autonomi, ciò che ci ispira è l'idea di lasciare al cervello artificiale la possibilità di imparare autonomamente come agire nel modo migliore per risolvere un determinato compito. Sfortunatamente, i cervelli artificiali non apprendono velocemente quanto i cervelli degli esseri viventi e richiedono migliaia o milioni di tentativi anche per risolvere semplici problemi.

In questa tesi, studiamo i metodi per approcciare il ciclo di percezione, ragionamento e azione con il generale approccio di apprendimento tramite interazione per risolvere diversi problemi di robotica come navigazione ed esplorazione autonoma. Questo paradigma di apprendimento è comunemente conosciuto come Apprendimento per Rinforzo [1]. In particolare, studiamo come le rappresentazioni delle informazioni, che vengono percepite dal robot, e delle azioni, decise dalla mente 
artificiale, possano essere imparate e come queste rappresentazioni possano semplificare il ragionamento e l'agire. Infine, studiamo come ricompensare la mente artificiale nel raggiungimento di risultati migliori e come questo si traduca nei problemi di robotica trattati.

Mostriamo come le forme non costrittive di conoscenze pregresse siano quelle da utilizzare in robotica, perchè non limitano l'apprendimento del comportamento ottimale, anzi migliorano l'abilità della mente artificiale di adattarsi a situazioni sconosciute, migliorano l'abilità di imparare velocemente con esperienza limitata e migliorano l'abilità di agire bene in presenza di incertezze. 


\section{Acknowledgments}

My $\mathrm{PhD}$ journey was an extremely tough triathlon race. The swim was chaotic and unpredictable, the waters were choppy and dark, and it was hard to find the correct direction to move forward. Then the bike began, and the speed started rising. Every day was busy and passed quickly, and, before realising it, I was getting closer and closer to the cut-off time. However, the run was yet to come! The clock started ticking faster, the energy started dropping swiftly, and the legs started hurting, but I could not stop. I was tired, but the race was not done!

With the finish line in sight, I want to thank all the people who supported me in this endeavour, from family to friends and from supervisors to colleagues. You made this thesis possible!

I want to thank Stefano, my promotor, for the great freedom given me to find my research direction, for allowing me to pursue it and for the support and feedback during the writing of this thesis. Thank you for all the time you dedicated to me despite your busy schedule. You will always be a major reference in my future career.

I want to thank Christoph, my co-promotor, for the amazing influence and support of my research in the last two years of my PhD. You helped me to define my road further. Thank you for all the time you dedicated to me. Your knowledge, skills and passion for research and teaching are a great source of inspiration. You will always be a major reference in my future career.

I want to thank Mannes, my supervisor, because my thesis would have been very different without you, and I would have not probably found my road. Your knowledge, support, and passion for research and teaching were crucial elements for this achievement. Thank you for all the time you made available for me, for our meetings and for the help with all the students committees I involved you in. You will always be a major reference in my future career. 
I want to thank Johan, my first daily supervisor, for all the support and help in the first two years of my PhD. You always kept me motivated when nothing was working, and you helped me to develop the critical attitude that is crucial for obtaining a PhD.

I want to thank Edwin, my second daily supervisor, for all the support in the final two years of my PhD. Thank you for all the practical help during the final phases of the Smart Tooling project and with the supervision of the students.

My PhD project was part of the Smart Tooling project aiming at cheaper and more efficient inspection and maintenance of industrial plants. I want to thank all the people involved for all their support, help and patience.

I would like to that Abeje and the Saxion researchers for the successful collaboration of the past two years, the kindness, the support, and the open-mindedness. I hope we will continue our collaboration together in the future.

I want to thank all the students I had the pleasure to supervise during their bachelor and master assignments. You have helped me to explore many different aspects of Reinforcement Learning and robotics and improve and refine my teaching and supervision skills. Thank you: Chantal, Idzard, Marta, Mohammed, Thijs, Nathalie, Xiangshuai, Khaled, Santiago, Jacco, Shijran, Atul, Dinah, Stefan, Rosanne, Ruben, Lior, Jacco, Yash, Luuk, Rob, Arnold, Saumya, Tim, Yannick, Jan, Jochem, Bastian, Paul, and Beck. I wish you all the best in your future careers.

I want to express my gratitude to all my colleagues in the Robotics and Mechatronics group for the pleasant atmosphere. A huge thank goes to Jolanda. You helped me in every aspect of my life in the Netherlands and always supported me in any situation. I want to thank Vincent for all the help during the tough, yet very instructive, years as a teaching assistant in Control Engineering, Gijs and Jan for all the tips and mentorship on the problem of "surviving inside university" and all the help with the students committees. I would also like to thank the technician Marcel, Sander, Gerben, and Henny for their support with all the technical and practical challenges I faced in my years at RaM.

I want to express my gratitude to the external committee members for reading my thesis and for the feedback that significantly improves this manuscript.

A huge thank goes to Beril and Andreas for being outstanding colleagues, collaborators, and, most importantly, outstanding friends. I know I can always count on you, and I am looking forward to working together again. 
In the past four years, I tried to enjoy every moment of freedom to recharge the batteries and refresh my mind. This would not have been possible without all my friends from the Aloha triathlon team. I will never forget all the races, funny events, training and evenings spent in the sport centrum. So, thank you: Koen, Daan, Bouke, Theo, Izak, Jasmin, Floor, and Eva and all the other members for the fantastic time we spent together.

I want to thank Martijn for being a great friend since my MSc thesis at RaM and for all the time spent having fun together.

Voglio ringraziare i miei più cari amici di Bologna, Alessio, Matteo, Andrea, Giacomo, Giulio, Filippo e tanti altri perchè, nonostante i $1300 \mathrm{~km}$ che ci hanno separato in questi anni, mi siete sempre rimasti vicini. Vi ringrazio per esserci stati e anche voi avete contribuito a questo grande traguardo.

Infine, un enorme ringraziamento va alla mia famiglia, in particulare a mia mamma Franca, a mio papà Fabio e a mia sorella Allegra, per l'illimmitato supporto quando tutto era difficile e niente funzionava, quando la pressione e la stanchezza erano alle stelle, ma anche ora all'inizio di un nuovo viaggio. Spero un giorno di potervi ripagare per tutto questo. Grazie! 



\section{Summary of Notation}

\section{Sets:}

$\mathcal{M}, \mathcal{X}, \mathcal{Y}$
$\mathbb{R}$
$\mathbb{N}$
$\Theta$
$(\mathcal{M}, \Sigma)$
$(\Theta, \Sigma, \operatorname{Pr})$
$\mathcal{S}$ or $\mathcal{S}^{e}$
$\mathcal{S}^{a}$
$\mathcal{A}$
$\mathcal{O}$
$\mathcal{A} a$
$\mathcal{B}$

Sets

Set of real numbers

Set of natural numbers

Set of possible outcomes or events

Measurable space. A measurable space is a pair $(M, \Sigma)$, where $\Sigma$ is a $\sigma$-algebra on $\mathcal{M}$

Probability space. A probability space is a triple $(\Theta, \Sigma, \operatorname{Pr})$ where $\operatorname{Pr}$ is a probability measure

Set of possible states of the environment or state space

Set of possible states of the agent

Set of possible actions or action space

Set of possible observations or observation space Set of possible abstract actions or abstract action space

Set of possible duration of abstract actions

\section{Functions:}

$$
\begin{aligned}
& f: \mathcal{X} \longrightarrow \mathcal{Y} \\
& f \circ g \\
& f(x ; \boldsymbol{\theta}) \\
& \|x\| \\
& \mu: \Sigma \longrightarrow[0, \infty]
\end{aligned}
$$$$
\text { Function } f \text { with domain } \mathcal{X} \text { and image in } \mathcal{Y}
$$

Composition of the function $f$ and $g$

A function of $x$ parametrised by the parameter vector $\boldsymbol{\theta}$

$L^{2}$ norm of $x$

Measure. A measure is a function on $(\mathcal{M}, \Sigma)$ 
$\operatorname{Pr}: \Sigma \longrightarrow[0,1]$

$\xi: \mathcal{M} \longrightarrow \mathbb{R}$

\section{Calculus:}

$\nabla_{x} y$

$\int f(x) d x$

$\int_{\mathcal{S}} f(x) d x$

\section{Probability Theory:}

$X, S, A, R$

$\operatorname{Pr}\{X=x\}$ or $p(x)$

$\mathbb{E}[X]$

$\sum_{x} p(x) x$

$\int_{\Theta} p(x) x d x$

$D_{\mathrm{KL}}(P, Q)$

Markov Decision Processes:

\section{$t$}

$s, s^{\prime}$

$a$

$r$

$\pi$

$\pi: \mathcal{S} \longrightarrow \mathcal{A}$

$\mathrm{T}: \mathcal{S} \times \mathcal{S} \times \mathcal{A} \longrightarrow[0,1]$

$\mathrm{R}: \mathcal{S} \times \mathcal{A} \longrightarrow \mathbb{R}$

$\pi(s)$

$\pi: \mathcal{S} \times \mathcal{A} \longrightarrow[0,1]$
Probability measure. A probability measure is a function on $(\Theta, \Sigma)$ such that $\operatorname{Pr}(\Theta)=1$ and $\operatorname{Pr}(\Sigma)=\sum_{i=1}^{N} \operatorname{Pr}\left(\Sigma_{i}\right)$ where $N$ is the number of elements of the $\sigma$-algebra $\Sigma$

The function $\xi$ is measurable if, for every real number $n$, the set $\{m \in \mathcal{M}: \xi(m)>n\}$ is measurable

Gradient of $y$ with respect to $x$

Integral over the entire domain of $x$

Integral with respect to $x$ over the set $\mathcal{S}$

Random variables. A random variable is measurable function on a probability space.

Probability that a random variable $X$ has value $x$

expected value of a random variable $X$

expected value of a discrete random variable $X$ expected value of a continuous random variable $X$

Kullback-Leibler divergence of $\mathrm{P}$ and $\mathrm{Q}$

Time step index. We always consider discrete time step $t \in \mathbb{N}$ if not stated otherwise

States

An action

A scalar reward

Policy or acting strategy or behaviour

Deterministic policy mapping states to actions

Transition function

Reward function

Deterministic policy $\pi$

Stochastic policy mapping states to actions to probability values 


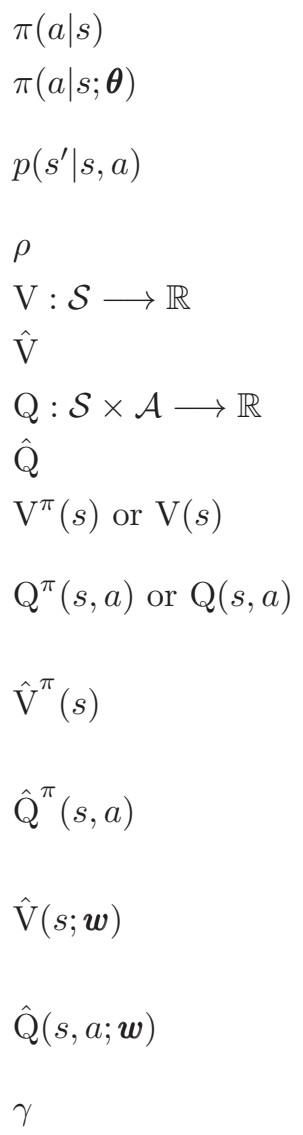

Special Operators:

$\propto$

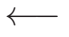

$\wedge$
Stochastic policy $\pi$

Stochastic policy parametrised by $\boldsymbol{\theta}$

Probability of transitioning to state $s^{\prime}$, from state $s$ taking action $a$

State distribution

State-value function or value function

Estimated value function

Action-value function

Estimated action-value function

Value of state $s$ under the policy $\pi$

Value of taking action $a$ in state $s$ under the policy $\pi$

Estimated value function of state $s$ under the policy $\pi$

Estimated value of taking action $a$ in state $s$ under the policy $\pi$

Estimated value function of the state $s$ parametrised by $\boldsymbol{w}$

Estimated action-value function of the stateaction pair $s, a$ parametrised by $\boldsymbol{w}$

Discount factor

Proportional-to operator

Assignment operator

Estimation operator 



\section{List of Acronyms}

$\begin{array}{ll}\text { RL } & \text { Reinforcement Learning } \\ \text { SRL } & \text { State Representation Learning } \\ \text { SLAM } & \text { Simultaneous Localization and Mapping } \\ \text { MDP } & \text { Markov Decision Process } \\ \text { POMDP } & \text { Partially Observable Markov Decision Process } \\ \text { RGB } & \text { Red Green Blue } \\ \text { LiDAR } & \text { Light Detection and Ranging } \\ \text { SMDP } & \text { Semi-Markov Decision Process } \\ \text { TD } & \text { Temporal Difference } \\ \text { DPG } & \text { Deterministic Policy Gradient } \\ \text { NN } & \text { Neural Network } \\ \text { ANN } & \text { Artificial Neural Network } \\ \text { MLP } & \text { Multi-Layer Perceptron } \\ \text { ReLU } & \text { Rectified Linear Unit } \\ \text { MSE } & \text { Mean Square Error } \\ \text { CNN } & \text { Convolutional Neural Network } \\ \text { RNN } & \text { Recurrent Neural Network } \\ \text { DQN } & \text { Deep Q-Network } \\ \text { DDPG } & \text { Deep Deterministic Policy Gradient } \\ \text { PPO } & \text { Proximal Policy Optimization } \\ \text { TRPO } & \text { Trust Region Policy Optimization } \\ \text { CPU } & \text { Central Processing Unit } \\ \text { GPU } & \text { Graphics Processing Unit } \\ \text { AE } & \text { Auto-Encoder } \\ \text { VAE } & \text { Variational Auto-Encoder }\end{array}$


FOV

$\mathrm{PCA}$

t-SNE

ROS

EKF

$\mathrm{RBPF}$

DWA

\section{Field Of View}

Principal Components Analysis

t-Distributed Stochastic Neighbor Embedding

Robot Operating System

Extended Kalman Filter

Rao-Blackwellized Particle Filter

Dynamic Window Approach 


\section{Contents}

Summary vii

Samenvatting $\quad$ ix

Sommario $\quad x i$

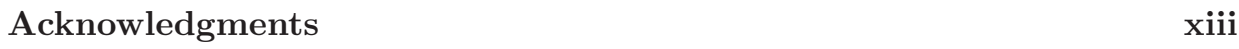

Summary of Notation xvii

List of Acronyms $\quad$ xxi

I Introduction and Theory 1

1 Introduction 3

1.1 Robotics and Artificial Intelligence . . . . . . . . . . . . 4

1.2 Motivations . . . . . . . . . . . . . . . . 5

1.2.1 The Reinforcement Learning Problem . . . . . . . . . . . . 5

1.2.2 Inspection and Maintenance . . . . . . . . . . . . 6

1.3 The Research . . . . . . . . . . . . . . . . . . 7

1.3.1 Contributions ................... 8

1.3.2 Publications. . . . . . . . . . . . . . . . 10

1.4 Thesis Outline . . . . . . . . . . . . . . . . . . 13

2 Markov Decision Processes and Reinforcement Learning 15

2.1 The Sequential Decision Making Problem . . . . . . . . . . . . . 16

2.1.1 Markov Decision Processes . . . . . . . . . . . 16 
2.1.2 Approaches for Solving Markov Decision Processes . . . . . 23

2.1.3 Partially Observable Markov Decision Processes . . . . . . 25

2.1.4 Semi-Markov Decision Processes . . . . . . . . . . 27

2.2 Reinforcement Learning . . . . . . . . . . . . . . . . . 30

2.2.1 Model-free and Model-based Reinforcement Learning . . . . 31

2.2.2 Value-based, Policy-based and Actor-Critic Methods . . . . 32

2.2.3 Approximating Value Functions and Policies . . . . . . . . 44

3 Deep Reinforcement Learning $\quad 47$

3.1 Artificial Neural Networks . . . . . . . . . . . . . . . . . . . . . . . 48

3.1.1 Feedforward Neural Networks . . . . . . . . . . . . . 48

3.1.2 Convolutional Neural Networks . . . . . . . . . . . . . . . 50

3.1.3 Recurrent Neural Networks . . . . . . . . . . . . . . . . 53

3.1.4 Deep Neural Networks . . . . . . . . . . . . . . . . 54

3.2 Reinforcement Learning with Deep NNs . . . . . . . . . . . . . . . 54

3.2.1 Deep Q-Network . . . . . . . . . . . . 56

3.2.2 Deep Deterministic Policy Gradient . . . . . . . . . . . 58

3.2.3 Twin Delayed Deep Deterministic Policy Gradient . . . . . 61

3.2.4 Proximal Policy Optimisation . . . . . . . . . . . . . 64

3.3 Representation Learning for Deep Reinforcement Learning . . . . . 66

3.3.1 Unsupervised Learning of State Representations with Neural Networks . . . . . . . . . . . . . . . 68

3.4 Deep Reinforcement Learning in Robotics . . . . . . . . . . . . . . 74

3.4.1 Challenges of Robotics Reinforcement Learning . . . . . . . 74

\section{State and Action Representation Learning 83}

4 State Representation Learning with Reward-shaped Priors $\quad 85$

4.1 Introduction . . . . . . . . . . . . . . . . . 86

4.2 Methodology ...................... 87

4.2.1 Proposed Approach . . . . . . . . . . . . . 87

4.2.2 Reward-shaped Priors . . . . . . . . . . . . 88

4.2.3 Neural Network Architectures and Training Regime . . . . 90

4.3 Experimental Design . . . . . . . . . . . . . . . . . . . . . . . . 91

4.3.1 Mobile Robot Navigation with Camera and LIDAR . . . . 91 
4.3.2 Reinforcement Learning Algorithm Settings . . . . . . . . . 91

4.3.3 Navigation Tasks in Different Environments . . . . . . . . . 92

4.3.4 Multi-targets State Representation . . . . . . . . . . . 92

4.3.5 Transfer Learning Experiments . . . . . . . . . . . . . 93

4.4 Results and Discussion . . . . . . . . . . . . . . . 93

4.4.1 Mobile Robot Navigation with Camera and LiDAR . . . . . 93

4.4.2 Navigation Tasks in Different Environments . . . . . . . . . 94

4.4.3 Multi-target Navigation . . . . . . . . . . . . . 98

4.4.4 Experiments in a Realistic Simulation Environment and on a Real Robot . . . . . . . . . . . . . . . 99

4.5 Conclusions ........................... 100

5 State Representation Learning with Robotics Priors in Continu$\begin{array}{lc}\text { ous Action Spaces } & 103\end{array}$

5.1 Introduction . . . . . . . . . . . . . . . . . . . 104

5.2 Related Work . . . . . . . . . . . . . . . . 106

5.3 Methodology ...................... 108

5.3.1 Proposed Approach . . . . . . . . . . . . . 108

5.3.2 Robotics Priors for Continuous Action Spaces . . . . . . . . 108

5.3.3 Neural Network Architectures . . . . . . . . . . . . . . . . 110

5.4 Experimental Design . . . . . . . . . . . . . . . . . 112

5.4.1 Mobile Robot Navigation with Multiple Sensor Modalities in Different Environments . . . . . . . . . . . . . . . 112

5.4 .2 Baselines ..................... 113

5.5 Results and Discussions . . . . . . . . . . . . . . . 114

5.5.1 Analysis of the Learned State Representations . . . . . . . 114

5.5 .2 Simulation Results . . . . . . . . . . . . . . . . . 115

5.5.3 Real-World Experiments . . . . . . . . . . . . . . . . 115

5.6 Conclusions . . . . . . . . . . . . . . . . . . . 118

6 State and Action Representation Learning with MDP Homomor$\begin{array}{lr}\text { phism Metrics } & 119\end{array}$

6.1 Introduction . . . . . . . . . . . . . . . . . . 120

6.2 Background . . . . . . . . . . . . . . . . . . . 121

6.2.1 MDP homomorphism . . . . . . . . . . . . 121 
6.3 Related Work . . . . . . . . . . . . . . . . . . . . . . . 123

6.3.1 Learning State Abstractions . . . . . . . . . . . . . . . 123

6.3 .2 Learning Action Abstractions . . . . . . . . . . . . . . . 124

6.3.3 Learning State and Action Abstractions . . . . . . . . . . . 124

6.4 Methodology . . . . . . . . . . . . . . . . . . . . . . . 124

6.4.1 State and Action Representation Learning . . . . . . . . 126

6.4 .2 Optimality of the Policies . . . . . . . . . . . . . 129

6.4.3 Neural Network Architectures . . . . . . . . . . . . . . . . 130

6.5 Experimental Design . . . . . . . . . . . . . . . . . . . 132

6.5.1 Grid-World . . . . . . . . . . . . . . . . . . . . . 132

6.5.2 Mobile Robot Navigation . . . . . . . . . . . . . . . . 134

6.5.3 Comparison of the Learned State Representations _. . . . 135

6.5.4 Comparison of the Learned Policies . . . . . . . . . . . . . 136

6.6 Results . . . . . . . . . . . . . . . . . . . . 137

6.6 .1 Grid-World . . . . . . . . . . . . . . . . . 137

6.6.2 Mobile Robot Navigation . . . . . . . . . . . . . . . 139

6.7 Discussion and Future Work . . . . . . . . . . . . . . . . . . 140

6.8 Conclusions . . . . . . . . . . . . . . . . . . . . 143

7 Towards Autonomous Pipeline Inspection with Hierarchical Reinforcement Learning 145

7.1 Introduction . . . . . . . . . . . . . . . . . . 146

7.2 Related Work . . . . . . . . . . . . . . . . . . . . . . . . 148

7.3 Autonomous Pipeline Inspection Robots . . . . . . . . . . . . . 148

7.3 .1 PIRATE Robot . . . . . . . . . . . . . . . . . . . 149

7.4 Methodology . . . . . . . . . . . . . . . . . 150

7.4.1 Reinforcement Learning for the PIRATE Robot . . . . . . . 150

7.4.2 Hierarchical RL and Policy Decomposition . . . . . . . . 151

7.5 Experimental Design . . . . . . . . . . . . . . . . . 155

7.5.1 Autonomous Navigation in Pipeline Networks . . . . . . . 155

7.5.2 Simulation Environment . . . . . . . . . . . . . . . 155

7.5.3 State Space and Neural Network Architectures . . . . . . . 155

7.5.4 Hyperparameters Tuning . . . . . . . . . . . . . . 156

7.5.5 Comparison of RL and HRL . . . . . . . . . . . 158

7.6 Results and Discussion . . . . . . . . . . . . . . . 158 
7.6 .1 Different Observation Groups . . . . . . . . . . . 158

7.6.2 Comparison of the RL and HRL Performances . . . . . . . 159

7.6.3 Comparison with Human Control . . . . . . . . . . . . . 161

7.6.4 Transferring the Policies to the Real Robot . . . . . . . . . 161

7.7 Conclusions . . . . . . . . . . . . . . . . 162

\section{Reward Shaping}

8 Entropy-Based Exploration for Mobile Robot Navigation: A LearningBased Approach $\quad 165$

8.1 Introduction . . . . . . . . . . . . . . . . . 166

8.2 Concise Introduction to Simultaneous Localisation and Mapping for Robotics . . . . . . . . . . . . . . 167

8.2.1 Rao-Blackwellized Particle Filter SLAM . . . . . . . . . . . 168

8.3 Methodology . . . . . . . . . . . . . . . . . . . 172

8.3.1 Proposed Approach _. . . . . . . . . . . . . 172

8.3 .2 Reward Function . . . . . . . . . . . . . . . 172

8.3.3 Reinforcement Learning Algorithm . . . . . . . . . . . . . . 174

8.4 Experimental Design . . . . . . . . . . . . . . . 176

8.4.1 Simulation Setup . . . . . . . . . . . . 176

8.4.2 Configuration of the RL and SLAM algorithms . . . . . . . 176

8.4.3 Training and Testing . . . . . . . . . . . 177

8.4.4 Real World Experiments . . . . . . . . . . . 178

8.5 Results and Discussion . . . . . . . . . . . . . . . . . . 180

8.5.1 Training and Testing . . . . . . . . . . 180

8.5.2 Real-world Experiments . . . . . . . . . . . . 183

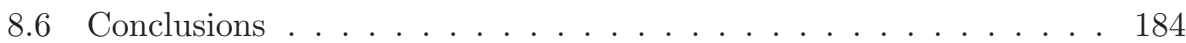

9 Reinforcement Learning for Mobile Robot Exploration $\quad 185$

9.1 Introduction . . . . . . . . . . . . . . . . 186

9.2 Related Work . . . . . . . . . . . . . . . . . . . . 187

9.3 Methodology . . . . . . . . . . . . . . . . . 189

9.3.1 Proposed approach . . . . . . . . . . . . . . 189

9.3.2 Exploration by Reward Shaping . . . . . . . . . . . . . 189

9.3.3 Neural networks architecture . . . . . . . . . . . . 193 
9.4 Experimental Design . . . . . . . . . . . . . . . . . . . . . 194

9.4 .1 Setup . . . . . . . . . . . . . . . . 194

9.4 .2 Baselines . . . . . . . . . . . . . . . . . . . . . 194

9.4 .3 Training and Testing . . . . . . . . . . . . . 195

9.5 Results and Discussions . . . . . . . . . . . . . . . . 196

9.5.1 Training Results . . . . . . . . . . . . . . . . . 196

9.5.2 Generalisation Results . . . . . . . . . . . . . . . . . 197

9.6 Conclusions . . . . . . . . . . . . . . . . . . . . . . . . 199

$\begin{array}{ll}\text { IV Conclusions and Discussions } & 201\end{array}$

10 Conclusions 203

10.1 The Answers . . . . . . . . . . . . . . . . . . . . . 204

10.1 .1 The Implications . . . . . . . . . . . . . . . 206

11 Discussions 209

11.1 Robotics Deep Reinforcement Learning . . . . . . . . . . . . . 210

11.2 The New Questions . . . . . . . . . . . . . . . . . . . . . 211

11.2.1 Generalisation to Unseen Situations and Meta-learning . . . 212

11.2.2 Efficient Exploration . . . . . . . . . . . . . . . . . . 213

11.2.3 Unifying Model-free and Model-based Reinforcement Learning213

11.2.4 Scaling to Real-world Problems . . . . . . . . . . . . . . . . 214

11.3 Final Thoughts . . . . . . . . . . . . . . . . . . . 215

$\begin{array}{ll}\text { Bibliography } & 217\end{array}$

Appendix A Proof of Proposition 2 233

$\begin{array}{ll}\text { Appendix B Supplementary Results } & 237\end{array}$ 


\section{Introduction and Theory}

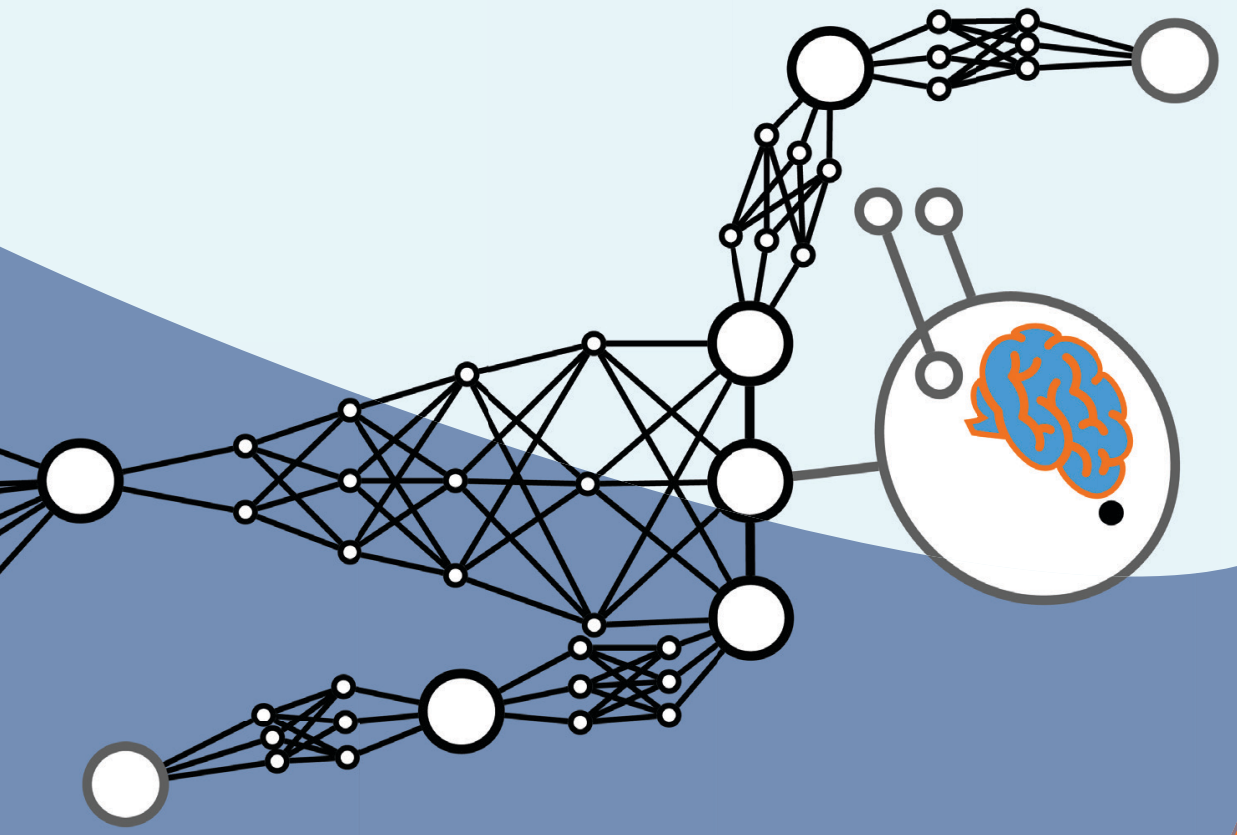





\section{Chapter 1}

\section{Introduction}

Uses promptos facit.

John Adams - Diary and Autobiography of John Adams

Improving skills and behaviours by practising is one of the most fundamental and powerful learning strategies that every animal and human experiences throughout its life. When designing artificial brains for autonomous robots, it is inspiring to proceed in the same way. Unfortunately, robots and artificial brains do not learn as fast as living beings and even solving simple problems requires thousands or millions of trials. In this thesis, we study how to reduce this gap to make robots learn new skills more efficiently and robustly. 


\subsection{Robotics and Artificial Intelligence}

Robotics research has tremendously progressed in the last decade, and robots can now be programmed and automated for solving different tasks, from everyday life simplest jobs, e.g. vacuum cleaning, or grass-cutting, to complex industrial applications, e.g. car assembly, smart warehouse, or inspection of plants. However, many steps have yet to be taken to achieve high degrees of cognitive and motoric intelligence and autonomy. These automated solutions require a vast amount of prior knowledge of the tasks and are often brittle in all the scenarios in which the robots have an imperfect and limited perception, inaccurate models of the world and uncertainties in the motion.

Artificial Intelligence, or AI, is the field of computer science striving to understand and design intelligence [2]. Many definitions exist, but for the purpose of this thesis, AI can be defined as "the study of the computations that make it possible to perceive, reason, and act" [3] in all the "activities that we associate with human thinking, activities such as decision-making, problem-solving, learning" [4]. When the problem requires not only perception and understanding of the world but also physical interaction with it, robots can be employed as the physical body of the AI agents. This often referred to in recent years as Embodied AI [5].

In this thesis, we focus on the study of the methods that tackle the perceivingreasoning-acting cycle with the general approach of learning by interacting with the environment for solving different robotics tasks, such as autonomous navigation, path planning and exploration. The computational paradigm of learning by interacting is commonly known as Reinforcement Learning [1]. In particular, we study how representations of the perceived information of the world can be learned and how these representations can be used to simplify reasoning and acting in Reinforcement Learning. Moreover, we study how to reward the acting entity to achieve better behaviours and how this translates to different robotics tasks. Eventually, we deal with all these challenges by using different forms of prior knowledge that do not limit the learning of the optimal behaviour ${ }^{1}$, but instead increase the learner's ability to adapt to different and unseen-a-priori situations, i.e. the generalisation, the ability to learn fast with limited experience, i.e. the sample efficiency, and the ability to behave well in the presence of uncertainties,

\footnotetext{
${ }^{1}$ Throughout this thesis, we assume that the learner does not know the optimal solution. However, we have knowledge about the environment or the task that we want to exploit by incorporating them into the learning process.
} 
i.e. the robustness of the behaviours learned.

\subsection{Motivations}

\subsubsection{The Reinforcement Learning Problem}

Learning from interaction is the simplest yet strongest learning approach that every living being practices, and this helps to improve throughout life. Most of what humans and animals learn is built on the concept of iteratively acting and improving the behaviour based on the consequences of the actions taken. For example, when a child tries to pick up a toy, does not know, neither needs to know, the exact physical laws behind, the exact weight of the toy nor its geometry, but can adapt the grip of the hand and adjust the strength of the arm until the toy is caught only based on the perception of how the world has changed after his previous actions. Thus, the learning of the best acting strategy for picking the toy up is steered by the success or failure of the previous attempts.

Reinforcement Learning is the computer science discipline aiming at solving, in the most general way, one of the most fundamental problems of science: optimal decision-making. Reinforcement Learning studies how to learn to act optimally by looking at the experiences collected while interacting with an unknown world, i.e. the environment. The learner, often called in this context the agent, does not know nor needs to know the perfect rules governing the world beforehand because it learns these rules and how to deal with them through the interaction with the environment. The agent chooses its actions using the perceived information from the environment, i.e. the state of the environment. Moreover, to improve its strategy, the agent receives a single measure, i.e. the reward, assessing the quality of the decision taken.

This natural and general way of learning through interaction can solve any decision-making problem. In the context of this thesis, we focus on the use of Reinforcement Learning in robotics for solving different navigation and exploration tasks.

\section{Representation Learning for Reinforcement Learning}

To take decisions, the agent can observe, in general, a limited portion of the environment. Additionally, this partial information received by the agent usually 
contains irrelevant information for making decisions. For example, when driving on the highway, human brains receive, through their vision systems, i.e. the eyes, high-dimensional and complex observations from the world (e.g. the position of other cars, their colour, or weather information). However, only a limited portion of this information is needed for safe driving. When learning behaviours from these high-dimensional sets of information, the agent must distinguish what is essential for making a decision and what is irrelevant. This step is a very complicated one and usually increases the learning time of the Reinforcement Learning agents. Instead of learning the optimal strategy directly from the high-dimensional observations of the world, it is possible to first extract the meaningful information from them, consequently reduce their dimensionality, and eventually learn the behaviour directly from this compressed set of information. The extraction of the relevant information, i.e. the features, can be obtained using hand-crafted feature-extraction algorithms (e.g. edge detection, or object detection), but, more interestingly, the agent can learn compact and low-dimensional representations of the observations. This is commonly called, in the context of Reinforcement Learning, State Representation Learning [6].

Moreover, when driving on the highway, the driver takes actions, such as taking an exit, that involves a series of lower-ranking actions such as steering, accelerating, and braking. These actions can be further decomposed into series of movements of arms and legs and again in a sequence of muscle contractions. When learning complicated tasks, it is possible to exploit the structure and hierarchy of the actions to simplify the overall learning process.

\subsubsection{Inspection and Maintenance}

The Smart Tooling project ${ }^{2}$ aims at automating industrial inspection and maintenance using autonomous robots in the context of petrochemical industries. Robots can be used to navigate, explore, clean, and monitor dangerous or impossible areas to reach for humans.

Pipelines networks are the fulcrum of the oil and gas industries and of all gas and water mains. These pipes must be periodically inspected to guarantee the safety and proper functioning of the plants. However, inspection is usually

\footnotetext{
${ }^{2}$ Smart Tooling is an Interreg Flanders-Netherlands project sponsored by the European Union focused on automation in the process industry: making maintenance safer, cheaper, cleaner, and more efficient by developing new robot prototypes and tools.
} 
a long, expensive and tedious procedure that requires the complete shut-down of the plants and, in the specific case of industrial pipelines, the removal of the insulation around the pipes. In the case of metal pipes, the inspection is currently performed from the outside using ultrasonic or magnetic probes that measure the wall thickness.

In the last two decades, inspection robotics has focused on designing new robots for in-pipe inspection. However, especially in the case of small diameter pipes, the mechatronics of these robots is complex, costly and with low operability [7]. While the design of these in-pipe robots has quickly progressed, many steps have yet to be taken to navigate and inspect complex pipes autonomously. In-pipe robots operate in a highly constrained environment, with limited sensing equipment, with moderate knowledge of the pipeline networks and often have to deal with unpredictable situations such as slippage due to the fluids that may be present in the pipes during the inspection. Most of these in-pipe robots have multiple degrees of freedom that make the design of an autonomous, robust and adaptable control architecture challenging.

Inspection missions can be various, such as navigating to specific target locations and inspect them, searching for defects in unknown pipe networks, and exploring and building the maps of the plants. Therefore, in this thesis, we focus on the study of the problem of autonomous navigation and exploration using Reinforcement Learning to lay the foundations for efficient pipe inspection with autonomous and intelligent robots.

\subsection{The Research}

In this thesis, we study the effect of different forms of prior knowledge in robotics Reinforcement Learning for solving different mobile robot navigation and exploration tasks, and we aim at answering the following research question:

What is "good" prior knowledge for robotics Reinforcement Learning for a) improving generalisation, b) sample efficiency, and c) robustness of the learned behaviours?

To answer this broad question, we specifically focus on all the aspects of the Reinforcement Learning process, namely state, action and reward. The study of 
these elements leads to the following sub-questions:

$\diamond$ How can one learn state representations by incorporating prior knowledge of the environment?

$\diamond$ How can one jointly learn state and action representations by integrating prior knowledge of state and action space?

$\diamond$ How can one learn optimal policies by exploiting prior knowledge of the problem structures?

$\diamond$ How can one learn optimal policies by shaping the reward function using prior knowledge of the maps of the environments?

\subsubsection{Contributions}

In this thesis, we focus on Reinforcement Learning in the context of robotics, starting from the study of different forms of state representation learning, action abstraction and action decomposition. Then, we study the effect of shaping the reward function in different navigation and exploration problems using the information contained in the maps of the training environments. Eventually, we reason on the problem of transferring the learned strategies using virtual simulators to real robots. These three main contributions are listed and briefly discussed below.

\section{Dimensionality Reduction for State and Action Space}

We propose novel approaches for incorporating task-specific knowledge in the state representation learning process that allow Reinforcement Learning agents to efficiently solve robot navigation tasks in the case of discrete action spaces in Chapter 4, and continuous action space in Chapter 5. Then, we study the problem of the state representation jointly together with the action representation in the case of discrete action spaces in Chapter 6. Eventually, we study the problem of hierarchical action representation in the case of a complex multi-joint robot for in-pipe inspection in Chapter 7. 


\section{Reward shaping for navigation and exploration of unknown environ- ments}

In the context of robot navigation, environment representations, i.e. maps, are key elements for most navigation techniques, e.g. potential-fields and $\mathrm{A}^{*}$ [8]. Usually, Simultaneous Localization and Mapping, or SLAM, algorithms [9] are used to build such maps. However, these may not always be available if the environments are not known a priori. Therefore, we focus on learning to navigate without the need of the map in Chapter 8, and learning to explore the environment to construct maps in Chapter 9. Both challenges can be efficiently solved by shaping the reward functions. We present two approaches for reward shaping using the map's information for improving the training efficiency, the generalisation to unseen a priori environments and the transfer of the policies learned in the simulation environment to real robots.

\section{Applications to real robots and transfer learning}

To optimally perform, a Reinforcement Learning agent requires a long training time. Moreover, through the training procedure, the agent tries behaviours that may be undesired (e.g. robot bumping into a wall). These two drawbacks make it often unfeasible to directly train a Reinforcement Learning agent on a real robot due to the cost of the hardware and its fragility. This problem is commonly addressed in literature as the curse of real-world samples [10]. Physics engines and simulators can be used to alleviate the cost of real-world experiments by learning strategies using simulated virtual environments and transfer them to real robots, i.e. transfer learning. When run on powerful computer clusters, simulators can also considerably speed up the collection of the experience sample with respect to real robots. Unfortunately, simulators rely on mathematical models ${ }^{3}$ (e.g. physical models, sensor models, actuator models) of the worlds that are never equal to reality. We call this simulation-to-reality gap. Thus, transfer learning is challenging and not straightforward in most applications. In this thesis, we reason on how behaviours should be learned to allow effective transfer learning.

\footnotetext{
${ }^{3}$ According to [11], "a mathematical model is representation in mathematical terms of the behaviour of real devices or objects."
} 


\subsubsection{Publications}

This thesis is built upon the following scientific publications ${ }^{4}$ :

[12] Nicolò Botteghi, Ruben Obbink, Daan Geijs, Mannes Poel, Beril Sirmacek, Christoph Brune, Abeje Mersha, and Stefano Stramigioli. Low-Dimensional State Representation Learning with Reward-shaped Priors. International Conference on Pattern Recognition, January 2021.

I am the sole first author of this paper. I conceived and evaluated the approach proposed in the paper and made the main contributions to the design of conceptual framework, its implementation, and paper writing. The next two authors (RO, DG) contributed to the algorithm implementation, data collection, and evaluation. The last five authors (MP, BS, CB, AM, SS) gave scientific advice and contributed to reviewing and improving the paper.

[13] Nicolò Botteghi, Khaled Alaa, Mannes Poel, Beril Sirmacek, Christoph Brune, Abeje Mersha, and Stefano Stramigioli. Low-Dimensional State Representation Learning with Robotics Priors in Continuous Action Spaces. International Conference on Intelligent Robots and Systems, September 2021.

I am the sole first author of this paper. I conceived and evaluated the approach proposed in the paper and made the main contributions to the design of conceptual framework, its implementation, and paper writing. The next author (KM) contributed to the algorithm implementation, data collection, and evaluation. The last five authors (MP, BS, CB, AM, SS) gave scientific advice and contributed to reviewing and improving the paper.

[14] Nicolò Botteghi, Mannes Poel, Beril Sirmacek, and Christoph Brune. LowDimensional State and Action Representation Learning with MDP Homomorphism Metrics. arXiv, July 2021.

I am the sole first author of this paper. I conceived and evaluated the approach proposed in the paper and made the main contributions to the design of conceptual framework, its implementation, and paper writing. The

\footnotetext{
${ }^{4}$ The complete list of publications of the author can be found at: https://scholar.google. com/citations?user $=$ Txd9FRYAAAAJ\&hl=en\&o $i=a o$
} 
last three authors (MP, BS, CB) gave scientific advice and contributed to reviewing and improving the paper.

[15] Nicolò Botteghi, Luuk Grefte, Mannes Poel, Beril Sirmacek, Christoph Brune, Edwin Dertien, and Stefano Stramigioli. Towards Autonomous Pipeline Inspection with Hierarchical Reinforcement Learning. Submitted to International Conference on Robot Intelligence, Technology and Applications, August 2021 .

I am the sole first author of this paper. I conceived and evaluated the approach proposed in the paper and made the main contributions to the design of conceptual framework, and paper writing. The next author (LG) contributed to the main algorithm implementation, data collection, and evaluation. The last five authors (MP, BS, CB, ED, SS) gave scientific advice and contributed to reviewing and improving the paper.

[16] Khaled Alaa*, Nicolò Botteghi*, Beril Sirmacek*, Mannes Poel, and Stefano Stramigioli. Towards Continuous Control for Mobile Robot Navigation: a RL and SLAM Based Approach. International Society for Photogrammery and Remote Sensing Congress, June 2019.

I share the first authorship with the indicated authors, all of which contributed equally to the design of the conceptual framework, its implementation, and paper writing. The last two authors (MP, SS) gave scientific advice and contributed to reviewing and improving the paper.

[17] Nicolò Botteghi, Beril Sirmacek, Khaled Alaa, Mannes Poel, and Stefano Stramigioli. On Reward Shaping for Mobile Robot Navigation: A Reinforcement Learning and SLAM Based Approach. arXiv, February 2020.

I am the sole first author of this paper. I conceived and evaluated the approach proposed in the paper and made the main contributions to the design of conceptual framework, and paper writing. The next two authors (BS, KM) contributed to the main algorithm implementation, data collection, and evaluation. The last two authors (MP, SS) gave scientific advice and contributed to reviewing and improving the paper. 
[18] Nicolò Botteghi, Khaled Alaa, Beril Sirmacek, and Mannes Poel. EntropyBased Exploration for Mobile Robot Navigation: A Learning-Based Approach. Planning and Robotics Workshop at International Conference on Automated Planning and Scheduling, October 2020.

I am the sole first author of this paper. I conceived and evaluated the approach proposed in the paper and made the main contributions to the design of conceptual framework, and paper writing. The next author (KM) contributed to the main algorithm implementation, data collection, and evaluation. The last two authors (BS, MP) gave scientific advice and contributed to reviewing and improving the paper.

[19] Nicolò Botteghi, Beril Sirmacek, Rob Schulte, Mannes Poel, and Christoph Brune. Reinforcement Learning helps SLAM: learning to build maps. International Society for Photogrammery and Remote Sensing Congress, August 2020.

I am the sole first author of this paper. I conceived and evaluated the approach proposed in the paper and made the main contributions to the design of conceptual framework, and paper writing. The next two authors (BS, RS) contributed to the main algorithm implementation, data collection, and evaluation. The last two authors (MP, CB) gave scientific advice and contributed to reviewing and improving the paper.

[20] Nicolò Botteghi, Rob Schulte, Beril Sirmacek, Mannes Poel, and Christoph Brune. Curiosity-driven Reinforcement Learning Agent for Mapping Unknown Indoor Environments. International Society for Photogrammery and Remote Sensing Congress, July 2021.

I am the sole first author of this paper. I conceived and evaluated the approach proposed in the paper and made the main contributions to the design of conceptual framework, and paper writing. The next two authors (RS, BS) contributed to the main algorithm implementation, data collection, and evaluation. The last two authors (MP, CB) gave scientific advice and contributed to reviewing and improving the paper.

* contributed equally. 


\subsection{Thesis Outline}

The thesis is structured in four main parts. Part I (Chapter 1-3) covers introduction and background. Part II (Chapter 4-6) presents the work related to state and action representation in the context of robotics Reinforcement Learning. Part III (Chapter 8, 9) contains the work related to reward shaping for navigation and exploration. Finally, Part IV (Chapter 10, 11) discusses the conclusions, the answers to the research questions, the findings of the research, and future research perspectives $^{5}$.

\footnotetext{
${ }^{5}$ Chapter 1,2 and 3 are original to this thesis, Chapter 4 contains an adaptation of the work published in [12] (see Section 1.3.2), Chapter 5 contains an adaptation of the work published in [13], Chapter 6 contains an adaptation of the work published in [14], Chapter 7 contains an adaptation of the work published in [15], Chapter 8 contains an adaptation of the work published in [16], [17] and [18], Chapter 9 contains an adaptation of the work published in [19] and [20], and Chapter 10 and 11 are original to this thesis.
} 


\section{Chapter 2}

\section{From Markov Decision Processes to Reinforcement learning}

The journey of a thousand miles begins with one step.

Lao Tzu

Learning by interacting with the world is a promising learning strategy for robots, but how can we do it in practice? How can we study the process of acting and observing the changes in the world? How can we improve the robot's behaviours based on that? The formalisation of the answers to these questions lies in the mathematical model of the Markov Decision Processes and in the Reinforcement Learning framework. In this chapter, we introduce the former and the latter. 


\subsection{The Sequential Decision Making Problem}

Most real-world problems cannot be solved with a single good decision, but require a, sometimes complicate, sequence of decisions, e.g. making coffee, a robot navigating to a given target location, obtaining a $\mathrm{PhD}$ title or finishing a triathlon race. In all these cases, each decision has an impact on the future, in the shortterm and the long-term and, only in the future, the quality of the decision can be assessed. For example, in a triathlon ${ }^{1}$ competition, to minimise the overall race time, it is essential to distribute the effort among the three disciplines properly. Swimming at maximum speed decreases the swimming time and costs energy that is not available anymore when cycling or running. Despite the time gain in the initial fraction, consuming too excessive energy during swimming is not a good decision in the long-term future for minimising the total race time. The relation between present and future and its uncertainties have to be taken into account for making the best decisions. We can study, analyse and solve any sequential decision problem with the very same mathematical framework: the Markov Decision Processes [21].

\subsubsection{Markov Decision Processes}

A Markov Decision Process, or MDP, [22] is a general framework for modelling any sequential decision making problem in which a goal has to be achieved with a sequence of decisions. The entity making decisions is called agent and the world in which the agent lives and interacts with is called environment. The agent, at each discrete time instant $t$, perceives information of the environment, i.e. its state and, based on this information, the agent interacts with the environment through its action. Each action changes the environment and, consequently, the next state. For each action taken, the agent receives a single scalar value that assesses the quality of the action taken, i.e. the reward, accordingly to a pre-defined goaldependent reward function. The agent-environment interaction scheme is shown in Figure 2.1.

The set of states of the environment $\mathcal{S}$ includes all the information the environment uses for determining what happens next given the action of the agent. For example, physical laws determining the robot motion when torque is applied

\footnotetext{
${ }^{1}$ Triathlon is an endurance sport where each athlete has to swim, cycle and run (in this exact order) over a course to complete the race.
} 


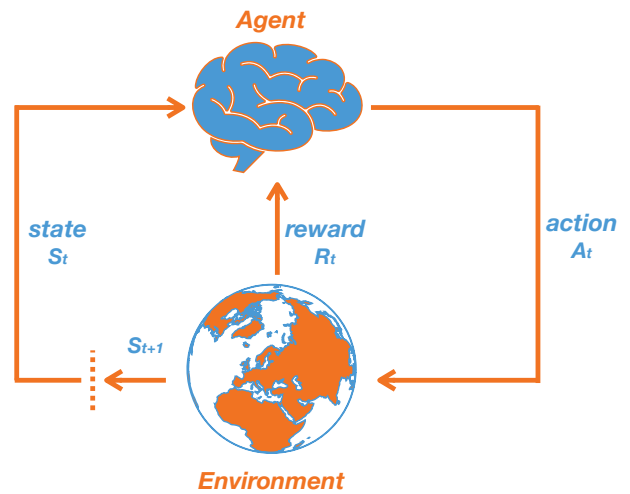

Figure 2.1: Agent-Environment interaction scheme for Markov decision processes.

to the actuator or the lines of code executed when, in a videogame, a button on the joystick is pressed. Additionally, we can identify the set of an agent's states $\mathcal{S}^{a}$ as the set of information the agent uses for choosing the next action to take. In MDPs, the states of the environment are directly observable by the agent and the agent's state space $\mathcal{S}^{a}$ is equivalent to the environment's state space $\mathcal{S}$. For example, the agent knows the true pose of the robot in a navigation task. Throughout Section 2.1.1 and 2.1.2, we assume the equivalence of environment state and agent state, and we refer to it as the state.

In the majority of real-world cases, the state of the environment is not directly observable. For example, the agent has no access to the true pose of the robot but can only obtain a noisy estimate given the sensors available. We speak in these cases of Partially Observable Markov Decision Processes, or POMDPs, and we treat them in Section 2.1.3. In the case of POMDPs, the agent's state space $\mathcal{S}^{a}$ and the environment's state space $\mathcal{S}$ do not coincide anymore.

In MDPs, actions can be low-level inputs to the robot's motors or high-level commands such as drinking coffee or tea for breakfast. We can define as actions any decision we want the agent to learn how to make and as states anything we think useful for learning such decisions. Consequently, the time steps of interaction between the agent and the environment do not necessarily need to be defined as fixed or periodic real-time intervals. Any time length of the intervals is allowed.

For each MDP, we can identify a transition function $\mathrm{T}: \mathcal{S} \times \mathcal{S} \times \mathcal{A} \longrightarrow[0,1]$; $\left(s^{\prime}, s, a\right) \longmapsto \mathrm{T}\left(s^{\prime}, s, a\right)=p\left(s^{\prime} \mid s, a\right)$ determining the evolution of the states. In the 
most general case, the environment dynamics of the MDP is stochastic, thus the transition function returns the probability $p\left(s^{\prime} \mid s, a\right)$ of reaching the next state $s^{\prime}$ when the action $a$ is taken in state $s$, as shown in Equation (2.1).

$$
\mathrm{T}\left(s^{\prime}, s, a\right)=p\left(s^{\prime} \mid s, a\right)=\operatorname{Pr}\left\{S_{t+1}=s^{\prime} \mid S_{t}=s, A_{t}=a\right\}
$$

where $\mathrm{T}$ is the transition function, $\operatorname{Pr}\left\{S_{t+1}=s^{\prime} \mid S_{t}=s, A_{t}=a\right\}$ is the probability that the next state random variable $S_{t+1}$ has value $s^{\prime}$ given that the current state variable $S_{t}$ has value $s$ and that the action taken $A_{t}$ has value $a$. Moreover, $\sum_{s^{\prime} \in \mathcal{S}} p\left(s^{\prime} \mid s, a\right)=1$ for all $s \in \mathcal{S}$ and $a \in \mathcal{A}$ to guarantee an acceptable probability value $\in[0,1]$. When the state is assumed fully observable, the present state contains sufficient information for determining what happens next in the environment, i.e. the future is independent of the past given the present [1]. This is the so-called Markov assumption.

We can also define the reward function $\mathrm{R}: \mathcal{S} \times \mathcal{A} \longrightarrow \mathbb{R} ;(s, a) \longmapsto \mathrm{R}(s, a)$ returning a scalar number for each transition to a state $s^{\prime}$ when the action $a$ is taken in state $s$. It is essential to highlight the underlying assumption on the reward function crucial for solving any MDP: each goal can be expressed as the maximisation of the expected cumulative reward [23]. If the agent can find the strategy that maximises the total future reward, then the agent has also optimally solved the sequential decision making process and achieved its goal.

We can now formally define a finite $\mathrm{MDP}^{2}$ as follows:

\section{Definition 2.1: Markov Decision Process}

A Markov Decision Process, or MDP, is a tuple $\langle\mathcal{S}, \mathcal{A}, \mathrm{T}, \mathrm{R}\rangle$ where $\mathcal{S}$ is the set of states, $\mathcal{A}$ is the set of actions, $\mathrm{T}: \mathcal{S} \times \mathcal{S} \times \mathcal{A} \longrightarrow[0,1] ;\left(s^{\prime}, s, a\right) \longmapsto$ $\mathrm{T}\left(s^{\prime}, s, a\right)=p\left(s^{\prime} \mid s, a\right)$ is the transition function and $\mathrm{R}: \mathcal{S} \times \mathcal{A} \longrightarrow$ $\mathbb{R} ;(s, a) \longmapsto R(s, a)$ is the reward function.

When phrasing a problem using the MDP framework, it is important to define the boundary between the agent and the environment clearly. This boundary defines what the agent can and cannot control. This bound does not usually coincide with the software-hardware bound, but, in practice, it is defined by the

\footnotetext{
${ }^{2}$ For the sake of simplicity, the theory presented holds for finite MDPs, i.e. MDPs with a finite number of states and actions and discrete-time interaction between agent and environment, but it can be extended to the continuous case as shown in [24] and [25].
} 
chosen characterisation of the states, actions and rewards for the specific task of interest. For example, if the goal of the agent is to steer a mobile robot to a given location, the hardware of a robot $^{3}$ is not considered as part of the agent, but only as parts of the environment.

Eventually, for each MDP, we can define a policy and a value function.

\section{Example 2.1: Grid-world}

One of the simplest examples of a finite MDP is the so-called grid-world. The grid-world, depicted in Figure 2.2, is a simple $3 \times 3$ chessboard, with eight free cells and one occupied cell, corresponding to an obstacle. The states of the environment correspond to the eight reachable cells of the chessboard. The agent, depicted with a robot, has to reach the target by taking actions. Only four actions are possible: move-up, move-down, move-left and move-right. In general, an MDP has a stochastic transition function $\mathrm{T}$, and the same action, taken in the same state, may not produce the same outcome, i.e. the next state may be different. For example, each action can succeed with a probability equal to 0.9 and fail with a probability of 0.1 . In the special case in which the actions of the agents always succeed, the MDP has a deterministic dynamics instead.

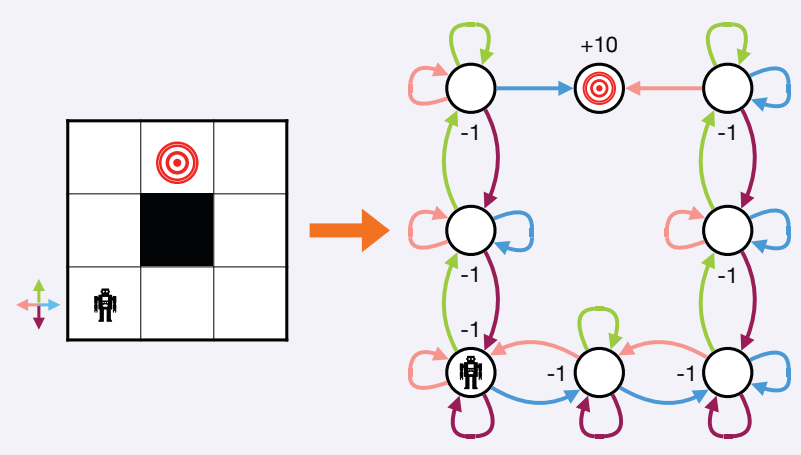

Figure 2.2: From a grid-world to a MDP.

A commonly used reward function for solving the grid-world is shown in

\footnotetext{
${ }^{3}$ The hardware of a robot is usually composed by its actuators, its sensors, its mechanical components and its electronics.
} 
Equation (2.2).

$$
\mathrm{R}(s, a)= \begin{cases}+10, & \text { if } s=s_{\text {target }} \\ -1, & \text { otherwise }\end{cases}
$$

where +10 reward points are given to the agent if the target state $s_{\text {target }}$ is reached and -1 for each action taken. The target state $s_{\text {target }}$ is also a terminal state, and, once the agent reaches it, the task is completed, and no further actions can be taken. While the first term of the reward function may be obvious, the -1 penalty is needed for reaching the target in the minimum amount of steps.

\section{Policy}

Intuitively, a policy is a strategy of acting in each of the states of the MDP. A policy $\pi_{d}: \mathcal{S} \longrightarrow \mathcal{A}$ is deterministic if, for a given state, the action chosen is always the same, i.e. $a=\pi(s)$. Conversely, a policy $\pi_{s}: \mathcal{S} \times \mathcal{A} \longrightarrow[0,1]$ is stochastic if the actions are chosen accordingly to a probability distribution, i.e. $a=\pi(a \mid s)$.

The optimal policy $\pi^{*}$, either stochastic or deterministic, is the policy that accumulates the highest total expected reward and therefore best achieves the goal of the agent. The total expected cumulative reward can be usually defined in two different ways: finite horizon and infinite horizon. In the first case, the total cumulative reward is specified in terms of the expected sum of rewards accumulated over a finite horizon of steps $N$ when following the policy $\pi$. This is shown in Equation (2.3).

$$
\mathbb{E}_{\pi}\left[\sum_{t=0}^{N} R_{t}\right]
$$

where $R_{t}$ is the instantaneous reward received at time-step $t$. Alternatively, we can use the infinite horizon formulation, where we compute the expectation of the sum of the discounted rewards obtained when following policy $\pi$ for an infinite amount of steps. This is shown in Equation (2.4).

$$
\mathbb{E}_{\pi}\left[\sum_{t=0}^{\infty} \gamma^{t} R_{t}\right]
$$

where $\gamma$ is the discount factor determining the importance of future rewards. If 
the discount factor $\gamma<1$, the sum converges to a finite value, i.e. a maximum value of the reward function exists. Alternatively, in infinite horizon problems, one has to shape the reward function to be bounded.

Finding the optimal policy is the purpose of any of the algorithm solving MDPs.

\section{Value Functions}

The value function $\mathrm{V}: \mathcal{S} \longrightarrow \mathbb{R} ;(s) \longmapsto \mathrm{V}(s)$, or value function ${ }^{4}$, evaluates the quality $^{5}$ of being in a state $s \in \mathcal{S}$. Similarly to the value function, the action-value function $^{6} \mathrm{Q}: \mathcal{S} \times \mathcal{A} \longrightarrow \mathbb{R} ;(s, a) \longmapsto \mathrm{Q}(s, a)$, evaluates the goodness $\mathrm{Q}(s, a)$ of taking a specific action $a \in \mathcal{A}$ in a state $s \in \mathcal{S}$. The estimation of the value function is the fundamental mechanism used by most of the methods for solving MDPs.

Formally, the value $\mathrm{V}^{\pi}(s)$ of a state $s$ can be expressed as the expected total return under the policy $\pi$, i.e. the sum over all the possible rewards that are collected from the current state $s$ if the agent follows the policy $\pi$ for an infinite amount of time steps. This is shown in Equation (2.5).

$$
\mathrm{V}^{\pi}(s)=\mathbb{E}_{\pi}\left[\sum_{k=0}^{\infty} \gamma^{k} R_{t+k} \mid S_{t}=s\right] \quad \text { for all } s \in \mathcal{S}
$$

where $R_{t}$ is the instantaneous reward received at time-step $t$ and $\gamma \in[0,1]$ is the discount factor weighting the trust in the future rewards. The value function in Equation (2.5) can be rewritten as:

$$
\begin{aligned}
\mathrm{V}^{\pi}(s) & =\mathbb{E}_{\pi}\left[R_{t}+\gamma R_{t+1}+\gamma^{2} R_{t+2}+\ldots \mid S_{t}=s\right] \\
& =\mathbb{E}_{\pi}\left[R_{t}+\gamma \mathrm{V}^{\pi}\left(s^{\prime}\right) \mid S_{t}=s\right] \\
& \downarrow \text { by definition of expected value } \\
& =\sum_{s^{\prime} \in \mathcal{S}} \mathrm{T}\left(s^{\prime}, s, a\right)\left(\mathrm{R}(s, a)+\gamma \mathrm{V}^{\pi}\left(s^{\prime}\right)\right) \\
& =\sum_{s^{\prime} \in \mathcal{S}} p\left(s^{\prime} \mid s, a\right)\left(\mathrm{R}(s, a)+\gamma \mathrm{V}^{\pi}\left(s^{\prime}\right)\right)
\end{aligned}
$$

where the value $\mathrm{V}^{\pi}(s)$ corresponds to the sum, over all possible next states $s^{\prime} \in$

\footnotetext{
${ }^{4}$ Sometimes referred to as state value function.

${ }^{5}$ The quality is defined by a single scalar value.

${ }^{6}$ Sometimes referred to as state-action value function.
} 
$\mathcal{S}$, of the instantaneous reward $\mathrm{R}(s, a)$ summed to the discounted value function $\gamma \mathrm{V}^{\pi}\left(s^{\prime}\right)$ of the next state $s^{\prime}$ and weighted by the transition probability $\mathrm{T}\left(s^{\prime}, s, a\right)$ of going in a certain $s^{\prime}$ when following the policy $\pi$ is state $s$.

The optimal policy $\pi^{*}$ is the one that achieves the highest expected reward in the future, and the value function is a way to estimate the expected future reward for a given policy. Thus, for all $s \in \mathcal{S}$, the value $\mathrm{V}^{\pi^{*}}(s)$ of state $s$ under the optimal policy $\pi^{*}$ is the highest value that can be obtained. Moreover, the optimal value function $\mathrm{V}^{*}$ satisfies the so-called Bellman Equation in Equation (2.7), i.e. the value of a state under the optimal policy is equal to the expected return obtained by taking the best action in that state. Solving the Bellman Equation for all the states and actions results in finding the optimal solution of the MDP. However, this is usually unfeasible in practice due to the high amount of computations and storage memory needed ${ }^{7}$. Additionally, solving the Bellman Equation requires perfect knowledge of the MDP transition and reward model, which is not often available in complex problems.

$$
\mathrm{V}^{*}(s)=\max _{a \in \mathcal{A}} \sum_{s^{\prime} \in \mathcal{S}} \mathrm{T}\left(s^{\prime}, s, a\right)\left(\mathrm{R}(s, a)+\gamma \mathrm{V}^{*}\left(s^{\prime}\right)\right)
$$

where the optimal value function, of a state $s \in \mathcal{S}, \mathrm{V}^{*}(s)=\mathrm{V}^{\pi^{*}}(s)$ under the optimal policy $\pi^{*}$ is equal to the expected return obtained when the best action in each state is chosen ${ }^{8}$.

From the optimal value function in Equation (2.7), it is possible to derive the optimal greedy deterministic policy as:

$$
\pi^{*}(s)=\arg \max _{a \in \mathcal{A}} \sum_{s^{\prime} \in \mathcal{S}} \mathrm{T}\left(s^{\prime}, s, a\right)\left(\mathrm{R}(s, a)+\gamma \mathrm{V}^{*}\left(s^{\prime}\right)\right)
$$

where optimal greedy deterministic policy $\pi^{*}(s)$ is the policy always selecting the action with the highest value of the $\operatorname{state}^{9}$.

Next to the value function, it is possible to define the action-value function $\mathrm{Q}: \mathcal{S} \times \mathcal{A} \longrightarrow \mathbb{R}$. Analogously to Equation (2.5), we can express the actionvalue function with respect to expected reward obtained by following a policy $\pi$

\footnotetext{
${ }^{7}$ We return on this topic in Section 2.2.3.

${ }^{8}$ The best action is simply the action with the highest value.

${ }^{9}$ This is done by the function $\arg \max (\cdot)$.
} 
in Equation (2.9).

$$
\mathrm{Q}^{\pi}(s, a)=\mathbb{E}_{\pi}\left[\sum_{k=0}^{\infty} \gamma^{k} R_{t+k} \mid S_{t}=s, A_{t}=a\right]
$$

Moreover, the optimal action-value function $\mathrm{Q}^{*}=\mathrm{Q}^{\pi^{*}}$ under the optimal policy $\pi^{*}$ can be derived as in Equation (2.10).

$$
\mathrm{Q}^{*}(s, a)=\sum_{s^{\prime} \in \mathcal{S}} \mathrm{T}\left(s^{\prime}, s, a\right)\left(\mathrm{R}(s, a)+\gamma \max _{a^{\prime}} \mathrm{Q}^{*}\left(s^{\prime}, a^{\prime}\right)\right)
$$

where $a^{\prime}$ indicates an action taken in the next state $s^{\prime}$ From the optimal actionvalue function $\mathrm{Q}^{*}$ in Equation (2.10), it is possible to derive the optimal greedy deterministic policy:

$$
\pi^{*}(s)=\arg \max _{a} \mathrm{Q}^{*}(s, a)
$$

Eventually, by looking at the optimal value function $\mathrm{V}^{*}$ (in Equation (2.7)) and the optimal action-value function $\mathrm{Q}^{*}$, in Equation (2.10), it is possible to deduce their relation:

$$
\mathrm{V}^{*}(s)=\max _{a \in \mathcal{A}} \mathrm{Q}^{*}(s, a)
$$

Despite the equivalence of the optimal value function $\mathrm{V}^{*}$ and optimal actionvalue function $\mathrm{Q}^{*}$, shown in Equation (2.12), in all the situations in which we have no knowledge of the MDP model, i.e. when $\mathrm{T}$ and $\mathrm{R}$ are unknown, the estimation of the action-value function $\mathrm{Q}$ is preferred to the estimation of the value function $\mathrm{V}$. This is because, unlike $\mathrm{V}^{*}(s)$ that requires the knowledge of the successor states and their values when computing $\mathrm{V}^{*}\left(s^{\prime}\right)$ (see Equation $(2.7)$ ), the estimation of $\mathrm{Q}^{*}(s, a)$ can be directly done without any knowledge of the environment dynamics, by simply selecting the action with the highest Q-value by means of $\max _{a^{\prime}} \mathrm{Q}^{*}\left(s^{\prime}, a^{\prime}\right)$ (see Equation (2.10)). We can derive the optimal policy $\pi^{*}$, by simply taking the action for which the action-value function $\mathrm{Q}$ is maximum, as shown in Equation (2.11).

\subsubsection{Approaches for Solving Markov Decision Processes}

The algorithms solving MDPs can be divided into two major categories based on the amount of knowledge the agent has of the underlying process. The first category includes all the algorithms that assume complete and perfect knowledge 
of the MDP model, i.e. the transition function $\mathrm{T}$ and the reward function $\mathrm{R}$. This collection of algorithms is often called dynamic programming. Because finding the optimal value function requires an exhaustive search by looking at all the possible future options weighted by their probability of occurrence, we look into finding a good approximation of the value function that is computationally trackable. Examples of dynamic programming algorithms are policy iteration [26], and value iteration $^{10}[21]$.

On the other side, the algorithms in the second category do not assume any knowledge on the transition function $\mathrm{T}$ and the reward function $\mathrm{R}$. Therefore, they require interaction with the environment through the agent's actions to collect state transition and reward samples and estimate the value functions and policies. Reinforcement Learning algorithms [1] belong to this category. Section 2.2 explains and discusses this collection of methods and why they are so useful in practice.

\section{Example 2.2: Optimal Value function and Policy for the Grid- World}

Consider the eight states MDP introduced in Example 2.2. The problem of finding the optimal policy for reaching the target boils down to the problem of finding the optimal value function, either $\mathrm{V}^{*}$ or $\mathrm{Q}^{*}$. The optimal greedy policy can be then derived accordingly to Equation (2.8), if $\mathrm{V}^{*}$ is estimated, or to Equation (2.11), if $\mathrm{Q}^{*}$ is estimated.

Suppose we have perfect knowledge of the transition function $\mathrm{T}$ and the reward function $\mathrm{R}$, it is possible to compute the optimal value function utilizing any dynamic programming algorithm (see Section 2.1.2). Alternatively, suppose neither $\mathrm{T}$ nor $\mathrm{R}$ is known, the estimation of the optimal value function can be done with any Reinforcement Learning algorithm that can handle discrete state and action spaces.

How does the optimal value function look like? This is shown in Figure 2.3, where each cell of the maze is colored accordingly to its value. The optimal policy is then derived by simply choosing the action with the highest value, as shown in Equation (2.11). It is evident that, in the states $(0,2)$ and $(0,1)$,

\footnotetext{
${ }^{10}$ Value iteration estimates the value function using the Bellman Equation (see Equation (2.7)) for all possible states of the MDP. Value iteration is guaranteed to find the optimal value function if sufficient iterations are performed. After estimating the value function, the optimal policy is derived using Equation (2.8).
} 
the best action is move-up, while in $(0,0)$ the best action is move-right.
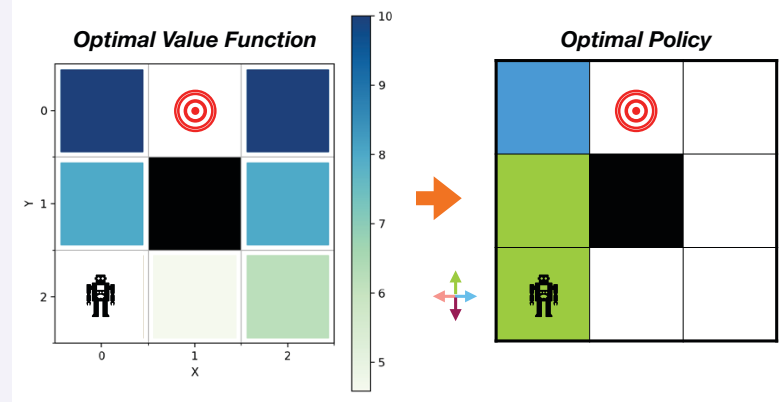

Figure 2.3: Optimal value function and optimal policy for the grid-world.

\subsubsection{Partially Observable Markov Decision Processes}

In Section 2.1.1, we introduced the MDP framework that leverages on the Markov assumption (Equation 2.1) and on the full observability of the environment state. However, in many interesting problems, the state of the environment is hidden and only a limited amount of information is perceivable by the agent. We call the information received by the agent in this context observations. The observation space $\mathcal{O}$ is the space of all the information the agent can perceive from the environment. The observations are received by the agent by means of an observation function $\Omega: \mathcal{O} \times \mathcal{S} \times \mathcal{A} \longrightarrow[0,1] ;(o, s, a) \longmapsto \Omega(o, s, a)=p(o \mid s, a)$. The observation function $\Omega$ returns the probability $p(o \mid s, a)$ of receiving an observation $o$ from a given state $s$ and action $a$. We talk in this case of Partially Observable Markov Decision Process, or POMDP. The agent-environment interaction scheme for POMPD is shown in Figure 2.4. We can formally define a POMDP as:

\section{Definition 2.2: Partially Observable Markov Decision Process}

A Partially Observable Markov Decision Process, or POMDP, is a tuple $\langle\mathcal{S}, \mathcal{A}, \mathcal{O}, \mathrm{T}, \Omega, \mathcal{R}\rangle$ where $\mathcal{S}$ is the set of states, $\mathcal{A}$ is the set of actions, $\mathcal{O}$ is the set of observations, $\mathrm{T}: \mathcal{S} \times \mathcal{S} \times \mathcal{A} \longrightarrow[0,1] ;\left(s^{\prime}, s, a\right) \longmapsto$ $\mathrm{T}\left(s^{\prime}, s, a\right)=p\left(s^{\prime} \mid s, a\right)$ is the transition function, $\Omega: \mathcal{O} \times \mathcal{S} \times \mathcal{A} \longrightarrow$ 
$[0,1] ;(o, s, a) \longmapsto \Omega(o, s, a)=p(o \mid s, a)$ is the observation function and $\mathrm{R}: \mathcal{S} \times \mathcal{A} \longrightarrow \mathbb{R} ;(s, a) \longmapsto \mathrm{R}(s, a)$ is the reward function.

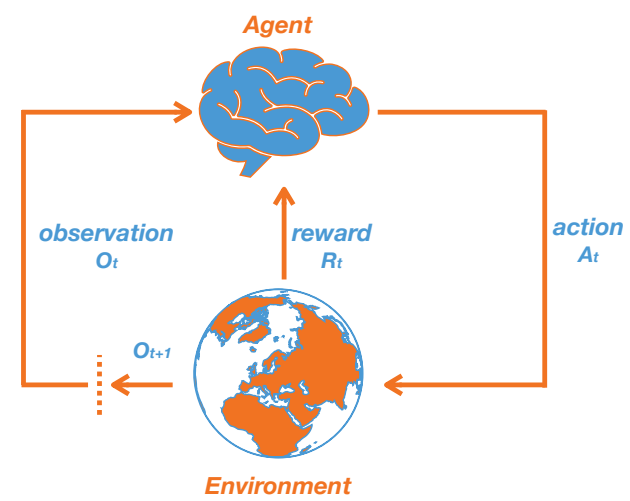

Figure 2.4: Agent-Environment interaction scheme for Partially Observable Markov decision processes.

Often a single observation, even though usually high-dimensional, does not contain enough clues to infer the state of the environment. For example, given a sensor reading, it may not be possible to know the robot's pose unequivocally or distinguish between two or more different situations. This ambiguity makes it difficult, or sometimes even impossible, to learn the optimal policy.

Because the state of the environment is not available anymore, the agent needs to build its state representation, i.e. its belief on how the state of the environment looks like, either explicitly or implicitly, to be able to solve the POMDP. In the most general formulation, the state of the agent at time $t$, i.e. $S_{t}^{a}$, can be defined as a function $f$ of the complete history of the observations coming from the environment and actions taken by the agent, as shown in Equation (2.13).

$$
S_{t}^{a}=f\left(O_{t}, O_{t-1}, \ldots, O_{t-n}, \ldots, O_{1}, O_{0}, A_{t-1}, A_{t-2}, \ldots, A_{t-1-n}, \ldots, A_{1}, A_{0}\right)
$$

Because of the partial and noisy information received, even in the case of complete history available, the agent's state space $\mathcal{S}^{a}$ may not coincide with the environment's state space $\mathcal{S}$. However, a good state representation contains all the 
relevant information for optimally solving the task. The function $f$ can be either hand-crafted, learned using function approximators or recursively estimated. SLAM algorithms commonly perform the recursive estimation of the belief [9]. For example, the robot's pose, i.e. its state ${ }^{11}$, is computed at each time step given the history of previous state estimates, sensor measurements, control actions and the map of the environment, if available.

\section{Example 2.3: Robot Navigation in Partially Observable Settings}

An agent controlling a robot for navigating and exploring the rooms of a building may receive multiple sensory readings (e.g. RGB camera images) from the environment that are similar to each other (e.g. pictures of white walls) but belong to different areas of the house or even different houses. In this case, the MDP framework does not hold anymore, and the POMDP framework has to be employed.

To solve this POMPD, intuitively, we need to find a good state representation for determining the robot's pose unequivocally in the building. The agent could rely only on observing the entire sequence of sensory readings and actions taken. By using the complete history, the agent may infer the state of the environment, i.e. the absolute location of the robot, and, therefore, it can choose the optimal actions. This can be done through learning methods or by maintaining a belief, i.e. a probability distribution $p\left(S_{t} \mid O_{t: 0}, A_{t-1: 0}\right)$, over the possible locations of the robot given the history of observations and actions.

For more information on POMPDs, we refer the reader to [9] and [22].

\subsubsection{Semi-Markov Decision Processes}

Complex MDPs cannot and should not be tackled by brute force approaches by recursively iterating over all possible states and actions. Real-world problems are intrinsically structured and these structures can be exploited to simplify the search for the optimal solution. In particular, we are interested in the extension of the Markov framework dealing with hierarchically structured problems: the

\footnotetext{
${ }^{11}$ For the sake of simplicity, we consider only the pose of the robot as relevant information for solving the task. However, there might be other relevant information in the state of the environment we would like to estimate, such as the battery level or the obstacle locations.
} 
Semi-Markov Decision Processe, or SMDP, [27].

\section{Example 2.4: Exploiting the Problem Structure}

We want to find the optimal policy for driving a car from point $\mathrm{A}$ to point $\mathrm{B}$ via the shortest path. Using the standard MDP approach, we can define low-level actions such as accelerating, braking, steering and shifting. We can solve this task in the original action space at the price of high computational complexity. We may need thousands or millions of actions to reach point B. However, we can also define high-level actions, such as drivestraight, turn-left or turn-right, where each of these action is composed by a combination of accelerating, braking, steering and shifting. These high-level actions can have variable duration in terms of time steps. Intuitively, the task in the new action space has a shorter horizon, and it can be optimally solved with a sequence of only five actions. This is shown in Figure 2.5.

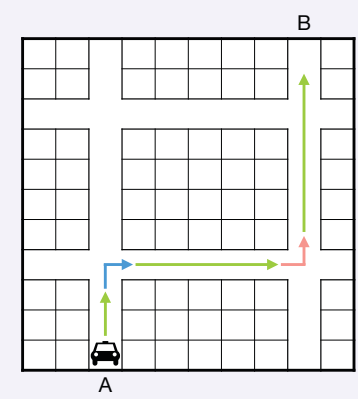

Figure 2.5: Policy with high-level actions.

Hierarchical approaches leverage on the concept of temporally extended or $a b$ stract actions that reduce the complexity and the horizon of the problems by replacing (long) sequences of low-level actions. The theory introduced for MDPs can be adapted to fit the SMDP framework by considering a random variable $B \geq 1$ equal to the number of time steps that an abstract action $a_{a}$ requires to finish when applied in state $s$ and terminating in $s^{\prime}$. Similarly to the MDP case, the transition function $\mathrm{T}: \mathcal{S} \times \mathcal{S} \times \mathcal{A}_{a} \times \mathcal{B} \longrightarrow[0,1] ;\left(s^{\prime}, s, a_{a}, b\right) \longmapsto \mathrm{T}\left(s^{\prime}, s, a_{a}, b\right)=p\left(s^{\prime} \mid s, a_{a}, b\right)$ returns the probability of transitioning to state $s^{\prime}$ when the abstract action $a_{a}$, lasting $b$ time steps, is taken in state $s$, where $\mathcal{B}$ is the set of all possible duration 
of the abstract actions. Analogously, the reward function $\mathrm{R}: \mathcal{S} \times \mathcal{A}_{a} \times \mathcal{B} \longrightarrow$ $\mathbb{R} ;\left(s, a_{a}, b\right) \longmapsto \mathrm{R}\left(s, a_{a}, b\right)$ can also be adapted from the MDP case by considering the time steps $b$ of duration of an abstract action. We can formally define a SMDP according to Definition 2.3.

\section{Definition 2.3: Semi-Markov Decision Process}

A Semi-Markov Decision Process, or SMDP, is a tuple $\left\langle\mathcal{S}, \mathcal{A}_{a}, \mathcal{B}, \mathrm{T}, \mathrm{R}\right\rangle$ where $\mathcal{S}$ is the set of states, $\mathcal{A}_{a}$ is the set of abstract actions, $\mathcal{B}$ is the set of all possible duration of the abstract actions, $\mathrm{T}: \mathcal{S} \times \mathcal{S} \times \mathcal{A}_{a} \times \mathcal{B} \longrightarrow$ $[0,1] ;\left(s^{\prime}, s, a_{a}, b\right) \longmapsto \mathrm{T}\left(s^{\prime}, s, a_{a}, b\right)=p\left(s^{\prime} \mid s, a_{a}, b\right)$ is the transition function and $\mathrm{R}: \mathcal{S} \times \mathcal{A}_{a} \times \mathcal{B} \longrightarrow \mathbb{R} ;\left(s, a_{a}, b\right) \longmapsto \mathrm{R}\left(s, a_{a}, b\right)$ is the reward function.

The value function $\mathrm{V}: \mathcal{S} \longrightarrow \mathbb{R}$ for a SMDP can also be derived from the value function in Equation (2.5). In Equation (2.14), we extend the value function formulation under the policy $\pi$ to take into account temporally extended actions lasting $b$ time steps.

$$
\begin{aligned}
\mathrm{V}^{\pi}(s) & =\mathbb{E}_{\pi}\left[\sum_{k=0}^{\infty} \gamma^{k} R_{t+k} \mid S_{t}=s\right] \\
& \left.=\mathbb{E}_{\pi}\left[\left(R_{t}+\gamma R_{t+1}+\ldots+\gamma^{b-1} R_{t+b-1}\right)+\left(\gamma^{b} R_{t+b}\right)\right) \mid S_{t}=s\right] \\
& =\sum_{s^{\prime} \in \mathcal{S}, b} \mathrm{~T}\left(s^{\prime}, s, \pi(s), b\right)\left(\mathrm{R}(s, \pi(s), b)+\gamma^{b} V^{\pi}\left(s^{\prime}\right)\right)
\end{aligned}
$$

Moreover, we can define the optimal value function $\mathrm{V}^{*}(s)=\mathrm{V}^{\pi^{*}}(s)$ as:

$$
\mathrm{V}^{*}(s)=\max _{a_{a}} \sum_{s^{\prime} \in \mathcal{S}, b} \mathrm{~T}\left(s^{\prime}, s, \pi(s), b\right)\left(\mathrm{R}(s, \pi(s), b)+\gamma^{b} V^{*}\left(s^{\prime}\right)\right)
$$

The action-value function $\mathrm{Q}$ for a given policy $\pi$ can be written as: (2.16).

$$
\mathrm{Q}^{\pi}\left(s, a_{a}\right)=\sum_{s^{\prime} \in \mathcal{S}, b} \mathrm{~T}\left(s^{\prime}, s, \pi(s), b\right)\left(\mathrm{R}(s, \pi(s), b)+\gamma^{b} \mathrm{Q}^{\pi}\left(s^{\prime}, \pi\left(s^{\prime}\right)\right)\right.
$$

Eventually, the optimal value function $\mathrm{Q}^{*}\left(s, a_{a}\right)=\mathrm{Q}^{\pi^{*}}\left(s, a_{a}\right)$ can be defined as:

$$
\mathrm{Q}^{*}\left(s, a_{a}\right)=\sum_{s^{\prime} \in \mathcal{S}, b} \mathrm{~T}\left(s^{\prime}, s, \pi(s), b\right)\left(\mathrm{R}(s, \pi(s), b)+\gamma^{b} \max _{a_{a}^{\prime}} \mathrm{Q}^{*}\left(s^{\prime}, \pi\left(s^{\prime}\right)\right)\right.
$$


For more information on SMDPs, we refer the reader to [22].

\subsection{Reinforcement Learning}

In many cases, the model of the MDP is not known beforehand, and the only way to learn the optimal policy is to interact with the environment and improve the behaviour based on the experience collected. The experience, in Reinforcement Learning, corresponds to the collection of samples of states, actions, next states and rewards, i.e. the tuple $\left(s, a, r, s^{\prime}\right)$. Because the agent has no knowledge of the underlying MDP model, i.e. $\mathrm{T}$ and $\mathrm{R}$, the method for collecting the experience samples is of crucial importance. A trade-off has to be found between exploring the environment and collecting new, and potentially better, samples, and exploiting the current optimal policy and collect higher rewards.

Algorithm 1 shows the general computational scheme that most Reinforcement Learning algorithms use for learning policies and value functions ${ }^{12}$. In particular, here we treat the case of episodic Reinforcement Learning, where $M$ is the number of training episodes ${ }^{13}$ of interaction with the environment and within each episode, the agent executes at most $N$ actions. Using the experience collected at each step the estimations of the model of the MDP, $\hat{T}$ and $\hat{R}$, the value functions $\hat{Q}$ or $\hat{V}$ and of the policy $\pi$ are performed ${ }^{14}$.

\footnotetext{
${ }^{12}$ This is also called training phase.

${ }^{13}$ We call episode a complete interaction-cycle with the environment that starts in an initial state $s_{\text {init }}$ and terminates when $N$ actions are taken or when a terminal state is reached.

${ }^{14}$ We use the symbol ${ }^{\wedge}$ when talking about the estimates to distinguish them from the true T, R, Q and V.
} 


\section{Pseudo-code 2.1: General Reinforcement Learning Loop}

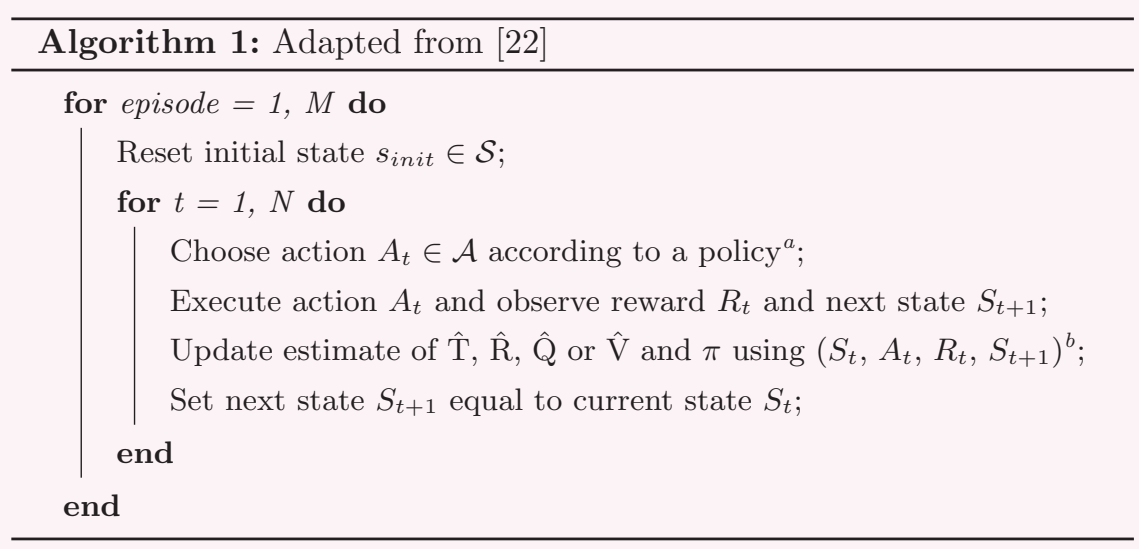

${ }^{a}$ The choice of the action is discussed later in Chapter 2 and in Chapter 3.

${ }^{b}$ The update rules for value functions and policies are discussed later in Chapter 2 and in Chapter 3.

\subsubsection{Model-free and Model-based Reinforcement Learning}

Many different Reinforcement Learning approaches exist, and all of them use the same experience tuple $\left(s, a, r, s^{\prime}\right)$ to learn the optimal policy. However, the experience can be used in different ways. Here, it is essential to highlight a major distinction among Reinforcement Learning algorithms concerning the use of the experience: model-free and model-based methods.

Model-free methods use the experience samples to directly estimate value functions, $\mathrm{V}$ or $\mathrm{Q}$, and policies $\pi$ without the need of any knowledge of the transition and reward functions, i.e. without knowledge of the model of the MDP. These methods have proven to be very successful in practice, but they are often datahungry and require many interaction samples to achieve good performances.

Model-based methods, on the other side, use the experience samples to estimate the transition functions $\hat{\mathrm{T}}$ and the reward functions $\hat{\mathrm{R}}$ and use these models to internally estimate value functions and policies without the need of further interaction with the environment ${ }^{15}$. These methods allow limited interaction with the environment and higher sample efficiency compared to model-free methods. However, learning accurate models of the MDP is, in most cases, really challenging,

\footnotetext{
${ }^{15}$ For instance, by solving the Bellman Equations (see Equation 2.7) and (2.10)
} 
and, when the model deviates too much from reality, the policy learned is far from the true optimal policy ${ }^{16}[10]$.

\subsubsection{Value-based, Policy-based and Actor-Critic Methods}

Another important distinction among Reinforcement Learning algorithms is made by how the policy is predicted. If the policy is derived from the estimation of the value function ${ }^{17}$, we talk about value-based methods, if the policy is directly sought, we talk about policy-based methods and if both the value function and the policy are explicitly estimated, we talk about actor-critic methods.

\section{Value-based Methods}

Value-based methods use the experience to estimate the value functions and consequently derive the policy, for example, as shown in Equation (2.11). Because the action-value function $\mathrm{Q}$ does not require knowledge of the MDP model and explicitly evaluates the contribution of the actions on the return ${ }^{18}$, the estimation of $\mathrm{Q}$ is often preferred to the estimation of $\mathrm{V}$.

All the methods presented in this section evaluate and estimate the optimal value functions. As discussed in the previous section, the optimal value function can only be evaluated if the agent follows the optimal policy. However, initially, the agent has no knowledge of the optimal policy and has to explore to collect new samples and evaluate their return by following a sub-optimal exploration policy. How can we estimate the optimal value function while following a sub-optimal policy? To answer this question, two approaches can be followed: on-policy and off-policy. On-policy approaches assume that the agent follows a nearly-optimal policy that is used for exploration and exploitation. Usually, these methods use a stochastic policy $\pi(a \mid s)$ that is gradually updated at each iteration in the direction of the optimal deterministic policy. Conversely, the off-policy approaches utilizes two different policies: a target policy $\pi$ that is improved at each iteration and a behaviour policy $\pi_{e}$ that is used to collect the samples ${ }^{19}$.

A very popular exploration policy is the so-called $\epsilon$-greedy policy. This is

\footnotetext{
${ }^{16}$ The policy is only as good as the estimated MDP model.

${ }^{17}$ This can be done, for example, using Equation (2.11).

${ }^{18}$ This is handy for control purposes since our ultimate goal is to find the best actions to solve a given problem.

${ }^{19}$ The target policy is used for exploitation, while the behaviour policy is used for exploration.
} 
shown by Algorithm 2. When following an $\epsilon$-greedy policy, the agent, at each time step, chooses an action either accordingly to a random policy ${ }^{20}$ with probability $\epsilon \in[0,1]$ or from the policy $\pi$ with probability $1-\epsilon$, where $\epsilon$ is a hyperparameter of the policy. For $\epsilon=0$, the agent follows the greedy deterministic policy by always selecting the action with the highest Q-value. In contrast, for $\epsilon=1$, the agent follows a random policy and each action is randomly sampled from the action space $\mathcal{A}$. Therefore, the value of $\epsilon$ is chosen to balance exploitation and exploration directly. The value of $\epsilon$ can be either chosen as a constant value, e.g. 0.3 , or can be decayed throughout the training episodes accordingly to any decay law, for example, a linear decay.

\section{Pseudo-code 2.2: $\epsilon$-greedy Policy}

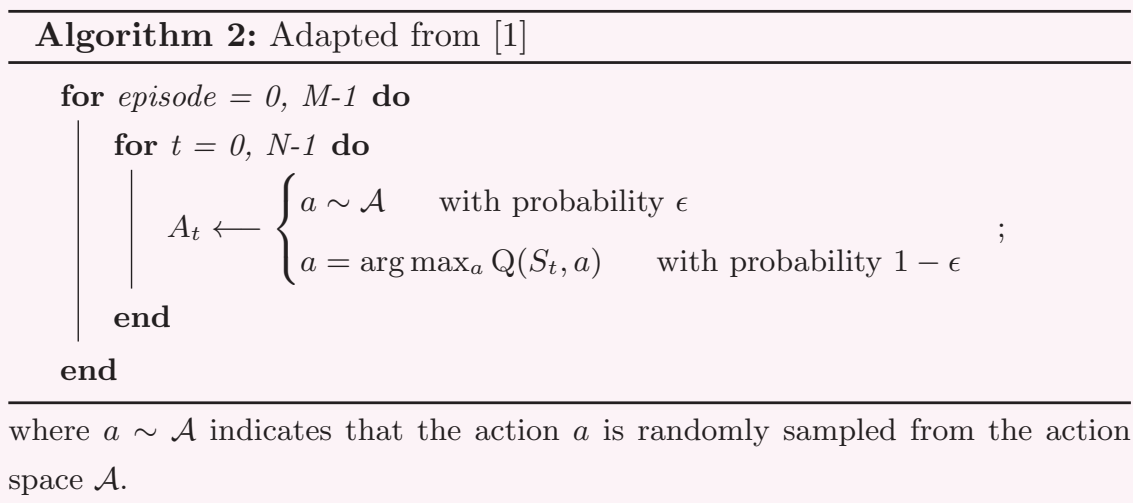

The value function is defined equal to the expected return under a given policy, see Equation (2.5). Thus, estimating the value functions boils down to estimating the return under a given policy. Again, we present two categories of methods that are very often used in practice: Monte Carlo and Temporal-Difference Learning.

Monte Carlo methods solve the problem of estimating the value function using the average sample returns of the training episodes. In particular, in the simplest case, the value function at $i^{t h}$-iteration $\hat{\mathrm{V}}_{i}\left(S_{t}\right)$ and the action-value function

\footnotetext{
${ }^{20}$ Choosing an action from a random policy is equivalent to sample an action from the action space randomly $\mathcal{A}$.
} 
$\hat{\mathrm{Q}}_{i}\left(S_{t}, A_{t}\right)$ an be estimated as in Equation (2.18) and (2.19) respectively ${ }^{21}$.

$$
\begin{gathered}
\hat{\mathrm{V}}_{i}\left(S_{t}\right) \longleftarrow \hat{\mathrm{V}}_{i-1}\left(S_{t}\right)+\alpha\left[\mathrm{G}_{t}-\hat{\mathrm{V}}_{i-1}\left(S_{t}\right)\right]=(1-\alpha) \hat{\mathrm{V}}_{i-1}\left(S_{t}\right)+\alpha \mathrm{G}_{t} \\
\hat{\mathrm{Q}}_{i}\left(S_{t}, A_{t}\right) \longleftarrow \hat{\mathrm{Q}}_{i-1}\left(S_{t}, A_{t}\right)+\alpha\left[\mathrm{G}_{t}-\hat{\mathrm{Q}}_{i-1}\left(S_{t}, A_{t}\right)\right]=(1-\alpha) \hat{\mathrm{Q}}_{i-1}\left(S_{t}, A_{t}\right)+\alpha \mathrm{G}_{t}
\end{gathered}
$$

where $\mathrm{G}_{t}$ is the return ${ }^{22}$ obtained from time step $t$ to the end of the episode and $\alpha$ is the learning rate, i.e. a constant parameter determining the magnitude of the update. Monte Carlo methods need to wait until the end of the episodes to make the value function updates because only then the return $\mathrm{G}$ is available to the agent. Moreover, these methods usually suffer due to the high variance of the return G. For more information on Monte Carlo methods, we refer the reader to $[1]$.

On the other side, we find Temporal-Difference Learning, or TD-learning, methods that do not require to wait the end of the episode to estimate the value function, but only until the next time step. Again, in the most simple case, the value function at the $i^{\text {th }}$-update $\hat{\mathrm{V}}_{i}\left(S_{t}\right)$ can be updated as in Equation (2.20). Similarly, the action-value function $\mathrm{Q}_{i}\left(S_{t}, A_{t}\right)$ can be updated as in Equation $(2.21)^{23}$.

$$
\begin{gathered}
\hat{\mathrm{V}}_{i}\left(S_{t}\right) \longleftarrow \hat{\mathrm{V}}_{i-1}\left(S_{t}\right)+\alpha\left[R_{t}+\gamma \hat{\mathrm{V}}_{i-1}\left(S_{t+1}\right)-\hat{\mathrm{V}}_{i-1}\left(S_{t}\right)\right] \\
\hat{\mathrm{Q}}_{i}\left(S_{t}, A_{t}\right) \longleftarrow \hat{\mathrm{Q}}_{i-1}\left(S_{t}, A_{t}\right)+\alpha\left[R_{t}+\gamma \hat{\mathrm{Q}}_{i-1}\left(S_{t+1}, A_{t+1}\right)-\hat{\mathrm{Q}}_{i-1}\left(S_{t}, A_{t}\right)\right]
\end{gathered}
$$

where the quantities $R_{t}+\gamma \hat{\mathrm{V}}\left(S_{t+1}\right)-\hat{\mathrm{V}}\left(S_{t}\right)$ and $R_{t}+\gamma \hat{\mathrm{Q}}\left(S_{t+1}, A_{t+1}\right)-\hat{\mathrm{Q}}\left(S_{t}, A_{t}\right)$ are usually referred to as TD-error and indicated with $\delta_{t}$. The TD-error determines the error on the current estimation of $\mathrm{Q}$ and the consequent direction and magnitude of the update of the estimate.

After estimating the value functions, we ultimately want to derive the optimal policy. For this reason, we focus from now on on the estimation of the actionvalue function $\mathrm{Q}$. We show here two popular TD-learning methods for control: SARSA [28] and Q-learning [29].

\footnotetext{
${ }^{21}$ Remembering the definition of value function and action-value function Equation (2.5) and (2.9) (see Section 2.1.1).

${ }^{22}$ The return is defined as $\mathrm{G}_{t}=\sum_{k=0}^{N-1} R_{t+k}$ or in discounted settings as $\mathrm{G}_{t}=\sum_{k=0}^{N-1} \gamma^{k} R_{t+k}$.

${ }^{23}$ Remembering the Bellman Equations in Equation (2.7) and (2.10).
} 
SARSA gets its name from the tuple $\left(S_{t}, A_{t}, R_{t}, S_{t+1}, A_{t+1}\right)$ used to update the $\hat{\mathrm{Q}}\left(S_{t}, A_{t}\right)$, as shown in Equation (2.22).

$$
\hat{\mathrm{Q}}_{i}\left(S_{t}, A_{t}\right) \longleftarrow \hat{\mathrm{Q}}_{i-1}\left(S_{t}, A_{t}\right)+\alpha\left[R_{t}+\gamma \hat{\mathrm{Q}}_{i-1}\left(S_{t+1}, A_{t+1}\right)-\hat{\mathrm{Q}}_{i-1}\left(S_{t}, A_{t}\right)\right]
$$

SARSA estimates, $\hat{\mathrm{Q}}^{\pi}(s, a)$ in on-policy fashion by assuming the agent is following the policy $\pi$, thus when estimating the value of the next state, $A_{t+1}$ is chosen accordingly to the current policy $\pi$. The complete iterative procedure of computation of $\hat{Q}$ is shown in Algorithm 3 assuming the agent is following an $\epsilon$-greedy exploration policy. Assuming an $\epsilon$-greedy policy, SARSA estimate of $\hat{\mathrm{Q}}$ converges with probability 1 to the true optimal action-value function $\mathrm{Q}^{*}$ if all the state-action pairs are infinitely visited ${ }^{24}$ [1]. Moreover, if the $\epsilon$ of the $\epsilon$ greedy policy decays, then the policy converges to the greedy deterministic policy $\pi(s)=\arg \max _{a} \hat{\mathrm{Q}}(s, a)$.

\footnotetext{
${ }^{24}$ In practice, it is sufficient to iterate only for a finite amount of steps.
} 


\section{Pseudo-code 2.3: SARSA on-policy TD-learning}

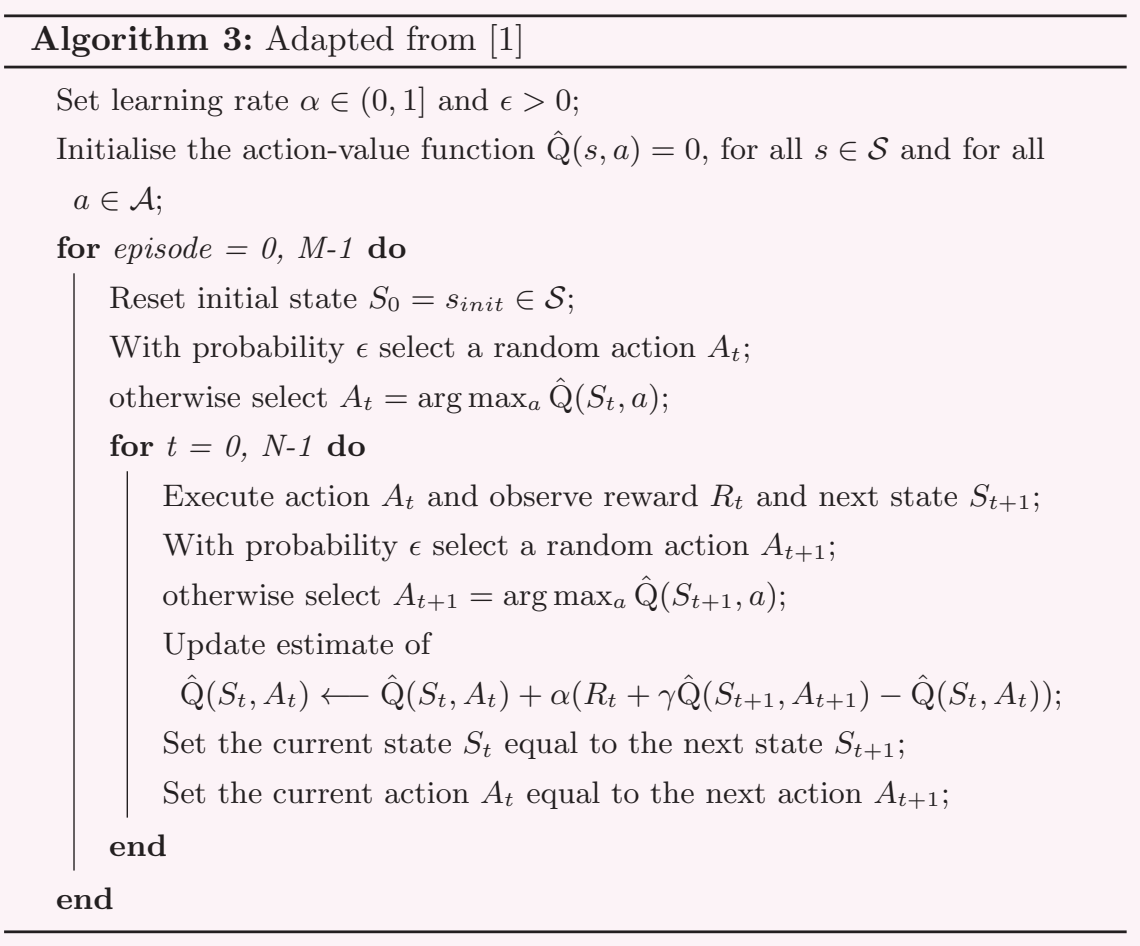

Q-learning is an off-policy TD-learning algorithm that estimates the actionvalue function $\mathrm{Q}$ independently of the policy followed by the agent. The update rule of the $\hat{Q}$ is now slightly different from the SARSA case, as shown in Equation (2.23), because it involves the greedy maximization over the actions of the value of the next state, i.e. the policy that is assumed to be followed for the update is the best policy. Q-learning converges with probability 1 to the optimal action-value function if all the state-action pairs are infinitely visited.

$$
\hat{\mathrm{Q}}_{i}\left(S_{t}, A_{t}\right) \longleftarrow \hat{\mathrm{Q}}_{i-1}\left(S_{t}, A_{t}\right)+\alpha\left[R_{t}+\gamma \max _{a} \hat{\mathrm{Q}}_{i-1}\left(S_{t+1}, a\right)-\hat{\mathrm{Q}}_{i-1}\left(S_{t}, A_{t}\right)\right]
$$

In Algorithm 4, the iterative algorithm used for estimating the action-value function is presented, assuming the agent is following an $\epsilon$-greedy exploration policy. 


\section{Pseudo-code 2.4: Q-learning off-policy TD-learning}

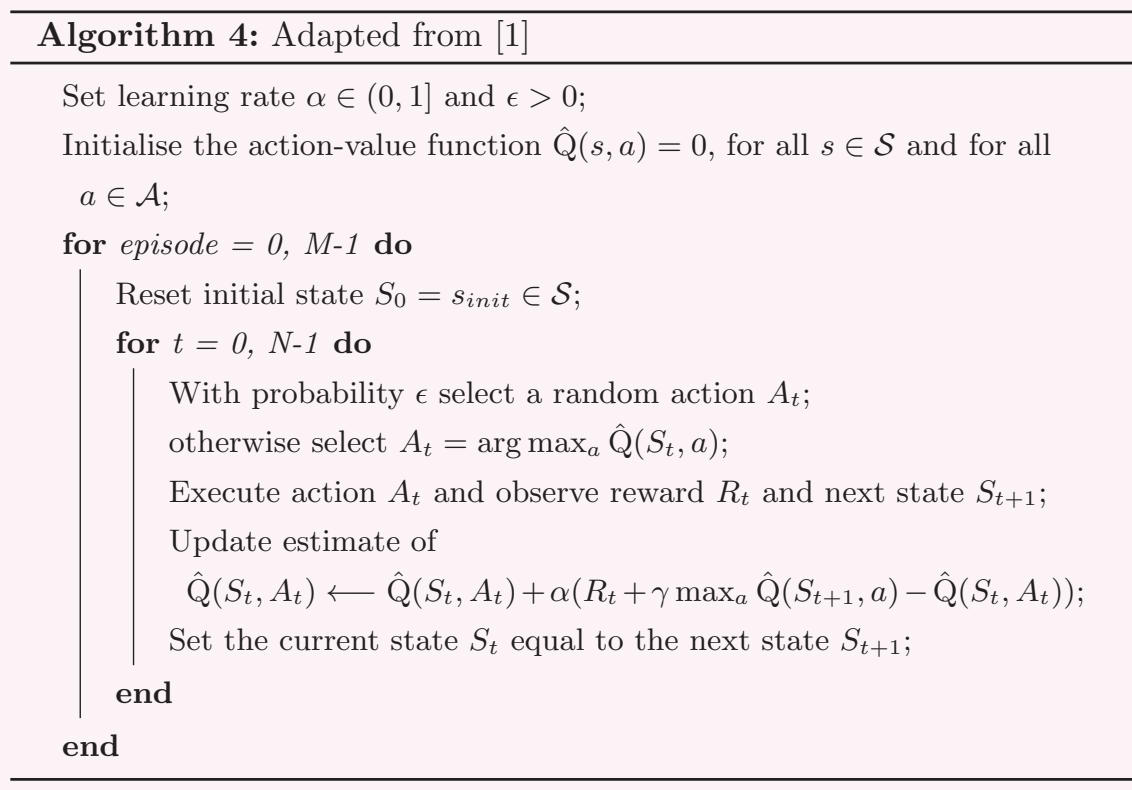

\section{Policy-based Methods}

In the value function-based methods, the policy is derived from the value function. Conversely, policy-based methods ${ }^{25}$ try to directly learn the optimal policy without the need of the value function. Despite the crucial role of the value functions in Reinforcement Learning, sometimes, these are very hard to learn [1].

We focus in this section on the case in which the policy is parametrised, as shown in Equation (2.24).

$$
\pi(a \mid s ; \boldsymbol{\theta})=\operatorname{Pr}\left\{A_{t}=a \mid S_{t}=s ; \boldsymbol{\theta}_{t}=\boldsymbol{\theta}\right\}
$$

where $\boldsymbol{\theta} \in \mathbb{R}^{d}$ is the parameter vector of dimension $d$. A common parametrisation in the case in which the action space is discrete and not too large is the soft-max distribution:

$$
\pi(a \mid s, \boldsymbol{\theta})=\frac{e^{h(s, a, \boldsymbol{\theta})}}{\sum_{i} e^{h\left(s, a_{i}, \boldsymbol{\theta}\right)}}
$$

\footnotetext{
${ }^{25}$ The theory presented in this section assumes a discrete state and action spaces. However, the theory can be easily extended to continuous state and action spaces.
} 
where $e$ is the base of the natural logarithm, $h(s, a, \boldsymbol{\theta}) \in \mathbb{R}$ is the function determining the preference of taking action $a$ is state $s$ given the current parametrisation of the policy $\boldsymbol{\theta}$. Alternatively, neural networks can be used to parametrise policies, and we treat this topic in Chapter 3. In this framework, the goal is to find the set of parameters $\boldsymbol{\theta}$ that maximises the performance of the policy. We can do this by ascending the gradient $\nabla$ of the performance measure $J(\boldsymbol{\theta})$ with respect to its parameter vector $\boldsymbol{\theta}$, as shown in Equation (2.26) for each new sample collected.

$$
\boldsymbol{\theta}_{t+1}=\boldsymbol{\theta}_{t}+\alpha \nabla_{\boldsymbol{\theta}_{t}} J\left(\boldsymbol{\theta}_{t}\right)
$$

where $\boldsymbol{\theta}_{t+1}$ is the parameter vector after update $t, \alpha$ is the learning rate determining the magnitude of the update on the current parameter vector $t$ and $\nabla_{\boldsymbol{\theta}_{t}} J\left(\boldsymbol{\theta}_{t}\right)$ is the gradient of the performance measure $J\left(\boldsymbol{\theta}_{t}\right)$ with respect to $\boldsymbol{\theta}_{t}$. Because the goal of the Reinforcement Learning agents is to maximise the return at the end of the run, we can define the performance measure $J(\boldsymbol{\theta})$ as:

$$
J(\boldsymbol{\theta})=\mathrm{V}^{\pi_{\boldsymbol{\theta}}}\left(s_{0}\right)
$$

where $\mathrm{V}^{\pi_{\boldsymbol{\theta}}}$ is the true value function under the policy $\pi_{\boldsymbol{\theta}}$. Updating the policy parameters $\boldsymbol{\theta}$ with respect to the performance measure $J$ changes the policy, consequently affects the action taken in each state, and eventually the state distribution $\rho: \mathcal{S} \rightarrow[0,1] ;(s) \longmapsto \rho(s)=p(s)$. However, while it is possible to compute changes in the action distribution and in the rewards received given a change of the policy parameters, the effect on the state distribution is usually unknown ${ }^{26}$. Usually, the state distribution $\rho(s)$, with $\rho(s) \geq 0$ and $\sum_{s} \rho(s)=1$, is chosen equivalent to the time spent in $s$. In the special case of on-policy algorithms, this is often referred to as on-policy state distribution. The on-policy state distribution can be defined as:

$$
\rho(s)=\frac{\eta(s)}{\sum_{s^{\prime} \in \mathcal{S}} \eta\left(s^{\prime}\right)} \quad \text { for all } s \in \mathcal{S}
$$

where $\eta(s)$ is the average number of times the state $s$ is visited in one episode under the policy $\pi$.

How can we estimate the gradient of the performance measure under unknown changes of the state distribution? This problem is solved by the policy gradient

\footnotetext{
${ }^{26}$ The environment's dynamics is assumed unknown in Reinforcement Learning.
} 
theorem [30]. The policy gradient theorem, shown in Theorem 2.1, analytically expresses the gradient of the performance measure with respect to the policy parameters $\theta$ without the need of the derivative of the unknown state distribution $\rho(s)$, see Equation (2.29).

$$
\nabla_{\boldsymbol{\theta}} J(\boldsymbol{\theta}) \propto \sum_{s \in \mathcal{S}} \rho(s) \sum_{a \in \mathcal{A}} \mathrm{Q}^{\pi_{\boldsymbol{\theta}}}(s, a) \nabla_{\boldsymbol{\theta}} \pi(a \mid s ; \boldsymbol{\theta})
$$

\section{Theorem 2.1: Policy Gradient}

$$
\begin{aligned}
\nabla_{\boldsymbol{\theta}} \mathrm{V}^{\pi_{\boldsymbol{\theta}}}(s) & =\nabla_{\boldsymbol{\theta}}\left[\sum_{a \in \mathcal{A}} \pi(a \mid s ; \boldsymbol{\theta}) \mathrm{Q}^{\pi_{\boldsymbol{\theta}}}(s, a)\right] \\
& =\sum_{a \in \mathcal{A}}\left[\nabla_{\boldsymbol{\theta}} \pi(a \mid s ; \boldsymbol{\theta}) \mathrm{Q}^{\pi_{\boldsymbol{\theta}}}(s, a)+\pi(a \mid s ; \boldsymbol{\theta}) \nabla_{\boldsymbol{\theta}} \mathrm{Q}^{\pi_{\boldsymbol{\theta}}}(s, a)\right] \\
& =\sum_{a \in \mathcal{A}}\left[\nabla_{\boldsymbol{\theta}} \pi(a \mid s ; \boldsymbol{\theta}) \mathrm{Q}^{\pi_{\boldsymbol{\theta}}}(s, a)+\pi(a \mid s ; \boldsymbol{\theta}) \nabla_{\boldsymbol{\theta}} \sum_{s^{\prime} \in \mathcal{S}} p\left(s^{\prime} \mid s, a\right) \mathrm{V}^{\pi_{\boldsymbol{\theta}}}\left(s^{\prime}\right)\right] \\
& =\sum_{a \in \mathcal{A}}\left[\nabla_{\boldsymbol{\theta}} \pi(a \mid s ; \boldsymbol{\theta}) \mathrm{Q}^{\pi_{\boldsymbol{\theta}}}(s, a)+\pi(a \mid s ; \boldsymbol{\theta}) \sum_{s^{\prime} \in \mathcal{S}} p\left(s^{\prime} \mid s, a\right) \nabla_{\boldsymbol{\theta}} \mathrm{V}^{\pi_{\boldsymbol{\theta}}}\left(s^{\prime}\right)\right] \\
& =\sum_{a \in \mathcal{A}}\left[\nabla_{\boldsymbol{\theta}} \pi(a \mid s ; \boldsymbol{\theta}) \mathrm{Q}^{\pi_{\boldsymbol{\theta}}}(s, a)+\pi(a \mid s ; \boldsymbol{\theta}) \sum_{s^{\prime} \in \mathcal{S}} p\left(s^{\prime} \mid s, a\right) \sum_{a^{\prime} \in \mathcal{A}}[\right. \\
& {\left.\left[\nabla_{\boldsymbol{\theta}} \pi\left(a^{\prime} \mid s^{\prime} ; \boldsymbol{\theta}\right) \mathrm{Q}^{\pi_{\boldsymbol{\theta}}}\left(s^{\prime}, a^{\prime}\right)+\pi\left(a^{\prime} \mid s^{\prime} ; \boldsymbol{\theta}\right) \sum_{s^{\prime \prime} \in \mathcal{S}} p\left(s^{\prime \prime} \mid s^{\prime}, a^{\prime}\right) \nabla_{\boldsymbol{\theta}} \mathrm{V}^{\pi_{\boldsymbol{\theta}}}\left(s^{\prime \prime}\right)\right]\right] } \\
& \downarrow \text { after repeated unrolling } \\
& =\sum_{s^{\prime} \in \mathcal{S}} \sum_{k=0}^{\infty} \operatorname{Pr}\left(s \longrightarrow s^{\prime}, k, \pi_{\boldsymbol{\theta}}\right) \sum_{a \in \mathcal{A}}\left[\nabla_{\boldsymbol{\theta}} \pi\left(a \mid s^{\prime}, \boldsymbol{\theta}\right) \mathrm{Q}^{\pi_{\boldsymbol{\theta}}}\left(s^{\prime}, a\right)\right]
\end{aligned}
$$

where $\operatorname{Pr}\left(s \longrightarrow s^{\prime}, k, \pi\right)$ is the probability of transitioning from $s$ to $s^{\prime}$ in $k$ steps under the policy $\pi_{\boldsymbol{\theta}}$. If we now consider, the performance measure defined in Equation (2.27), we can derive the policy gradient theorem in 
Equation (2.29).

$$
\begin{aligned}
\nabla_{\boldsymbol{\theta}} J(\boldsymbol{\theta}) & =\nabla_{\boldsymbol{\theta}} \mathrm{V}^{\pi_{\boldsymbol{\theta}}}\left(s_{0}\right) \\
& =\sum_{s \in \mathcal{S}}\left(\sum_{k=0}^{\infty} \operatorname{Pr}\left(s_{0} \longrightarrow s, k, \pi_{\boldsymbol{\theta}}\right)\right) \sum_{a \in \mathcal{A}}\left[\nabla_{\boldsymbol{\theta}} \pi(a \mid s ; \boldsymbol{\theta}) \mathrm{Q}^{\pi_{\boldsymbol{\theta}}}(s, a)\right] \\
& =\sum_{s \in \mathcal{S}} \eta(s) \sum_{a \in \mathcal{A}}\left[\nabla_{\boldsymbol{\theta}} \pi(a \mid s ; \boldsymbol{\theta}) \mathrm{Q}^{\pi_{\boldsymbol{\theta}}}(s, a)\right] \\
& =\sum_{s^{\prime} \in \mathcal{S}} \eta\left(s^{\prime}\right) \sum_{s \in \mathcal{S}} \frac{\eta(s)}{\sum_{s^{\prime} \in \mathcal{S}} \eta\left(s^{\prime}\right)} \sum_{a \in \mathcal{A}}\left[\nabla_{\boldsymbol{\theta}} \pi(a \mid s ; \boldsymbol{\theta}) \mathrm{Q}^{\pi_{\boldsymbol{\theta}}}(s, a)\right] \\
& =\sum_{s^{\prime} \in \mathcal{S}} \eta\left(s^{\prime}\right) \sum_{s \in \mathcal{S}} \rho(s) \sum_{a \in \mathcal{A}}\left[\nabla_{\boldsymbol{\theta}} \pi(a \mid s ; \boldsymbol{\theta}) \mathrm{Q}^{\pi_{\boldsymbol{\theta}}}(s, a)\right] \\
& \propto \sum_{s \in \mathcal{S}} \rho(s) \sum_{a \in \mathcal{A}}\left[\nabla_{\boldsymbol{\theta}} \pi(a \mid s ; \boldsymbol{\theta}) \mathrm{Q}^{\pi_{\boldsymbol{\theta}}}(s, a)\right]
\end{aligned}
$$

where $\eta(s)$ is the average number of time the state $s$ is visited in one episode under the policy $\pi_{\boldsymbol{\theta}}$ and $\rho(s)$ is the on-policy state distribution defined according to Equation (2.28). Therefore, the gradient $\nabla_{\boldsymbol{\theta}} J(\boldsymbol{\theta})$ of the performance measure $J$ can be computed without the need of the gradient of the unknown state distribution $\rho(s)$.

The policy gradient theorem poses the condition that policy $\pi(a \mid s ; \boldsymbol{\theta})$, despite its parametrisation, must be differentiable with respect to its parameter vector $\boldsymbol{\theta}$, i.e. $\nabla_{\boldsymbol{\theta}} \pi(a \mid s ; \boldsymbol{\theta})$ exists for all $s \in \mathcal{S}, a \in \mathcal{A}$ and $\boldsymbol{\theta} \in \mathbb{R}^{d}$. Moreover, to ensure enough exploration and collection of samples, the policy $\pi(a \mid s ; \boldsymbol{\theta}) \in(0,1)$ is usually stochastic.

REINFORCE [31] is one of the most widely-used Reinforcement Learning algorithms leveraging on policy gradient theorem. Equation (2.29) can be rewritten in terms of expectation to estimate the gradient of the policy given a set of samples. 
This is shown in Equation (2.32).

$$
\begin{aligned}
\nabla_{\boldsymbol{\theta}} J(\boldsymbol{\theta}) & \propto \sum_{s \in \mathcal{S}} \rho(s) \sum_{a \in \mathcal{A}} \mathrm{Q}^{\pi_{\boldsymbol{\theta}}} \nabla_{\boldsymbol{\theta}} \pi(a \mid s ; \boldsymbol{\theta}) \\
& =\mathbb{E}_{\pi_{\boldsymbol{\theta}}}\left[\sum_{a \in \mathcal{A}} \mathrm{Q}^{\pi_{\boldsymbol{\theta}}}\left(S_{t}, a\right) \nabla_{\boldsymbol{\theta}} \pi\left(a \mid S_{t} ; \boldsymbol{\theta}\right)\right] \\
& =\mathbb{E}_{\pi_{\boldsymbol{\theta}}}\left[\sum_{a \in \mathcal{A}} \pi\left(a \mid S_{t} ; \boldsymbol{\theta}\right) \mathrm{Q}^{\pi_{\boldsymbol{\theta}}}\left(S_{t}, a\right) \frac{\nabla_{\boldsymbol{\theta}} \pi\left(a \mid S_{t} ; \boldsymbol{\theta}\right)}{\pi\left(a \mid S_{t} ; \boldsymbol{\theta}\right)}\right] \\
& =\mathbb{E}_{\pi_{\boldsymbol{\theta}}}\left[\mathrm{Q}^{\pi_{\boldsymbol{\theta}}}\left(S_{t}, A_{t}\right) \frac{\nabla_{\boldsymbol{\theta}} \pi\left(A_{t} \mid S_{t} ; \boldsymbol{\theta}\right)}{\pi\left(A_{t} \mid S_{t} ; \boldsymbol{\theta}\right)}\right] \quad\left(a \text { is replaced by } A_{t} \sim \pi_{\boldsymbol{\theta}}\right) \\
& =\mathbb{E}_{\pi_{\boldsymbol{\theta}}}\left[\mathrm{G}_{t} \frac{\nabla_{\boldsymbol{\theta}} \pi\left(A_{t} \mid S_{t} ; \boldsymbol{\theta}\right)}{\pi\left(A_{t} \mid S_{t} ; \boldsymbol{\theta}\right)} \quad\left(\mathbb{E}_{\pi_{\boldsymbol{\theta}}}\left[G_{t} \mid S_{t}, A_{t}\right]=\mathrm{Q}^{\pi_{\boldsymbol{\theta}}}\left(S_{t}, A_{t}\right)\right)\right.
\end{aligned}
$$

We can then compute the parameters update using stochastic gradient ascent, as shown in Equation (2.33).

$$
\begin{aligned}
\boldsymbol{\theta}_{t+1} & =\boldsymbol{\theta}_{t}+\alpha \mathrm{G}_{t} \frac{\nabla_{\boldsymbol{\theta}} \pi\left(A_{t} \mid S_{t} ; \boldsymbol{\theta}\right)}{\pi\left(A_{t} \mid S_{t} ; \boldsymbol{\theta}\right)} \\
& =\boldsymbol{\theta}_{t}+\alpha \mathrm{G}_{t} \nabla_{\boldsymbol{\theta}} \ln \pi\left(A_{t} \mid S_{t} ; \boldsymbol{\theta}\right)
\end{aligned}
$$

where $\alpha$ is the learning rate chosen to scale the magnitude of the update of the parameters. The whole REINFORCE algorithm is shown in Algorithms 5. 


\section{Pseudo-code 2.5: REINFORCE}

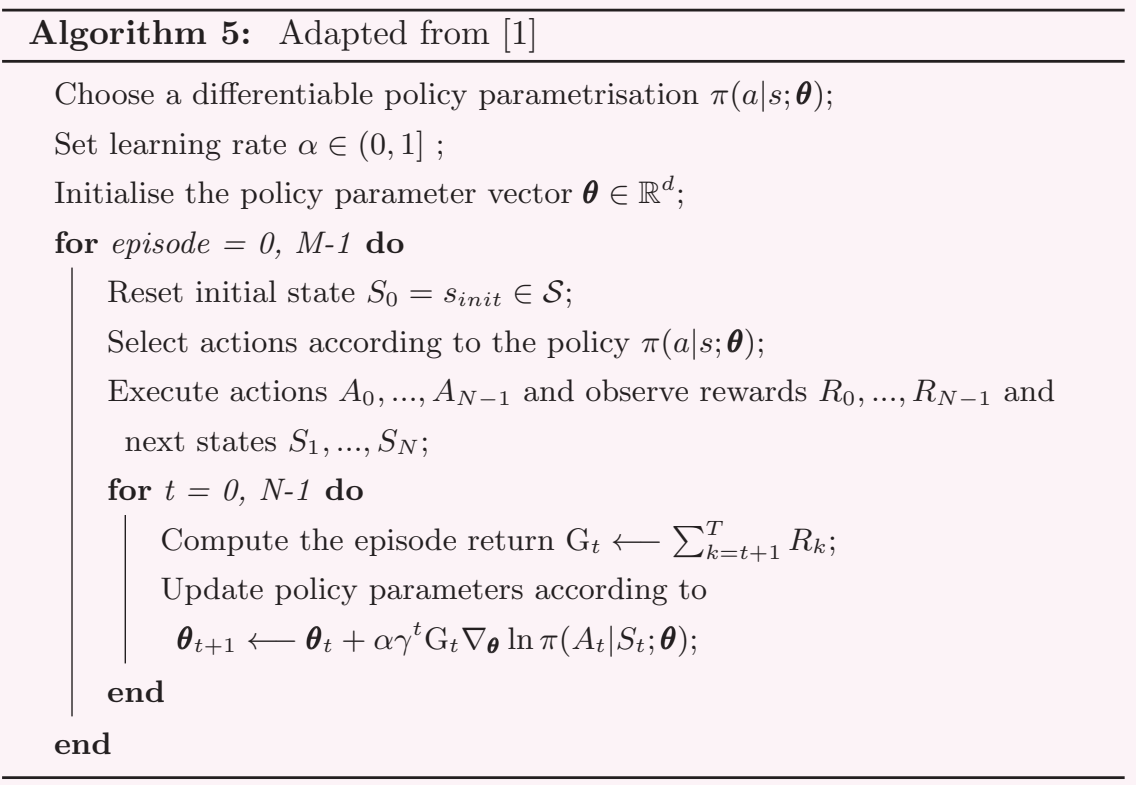

\section{Actor-Critic Methods}

The third class of algorithms combines the benefits of the value function and policy-based methods. Actor-critic algorithms learn either a policy, i.e. the actor, acting entity, and a value function, i.e. the critic, assessing the actor's performance and adjusting the policy in relation to its performance. This approach allows the reduction of the variance introduced by the return $\mathrm{G}$ by adding an explicit estimation of the value function $\hat{V}$ or the action-value function $\hat{Q}$ via temporal-difference learning. The policy parameters $\boldsymbol{\theta}$ are adjusted with gradient ascent accordingly to Equation (2.34) and (2.35) in the case the value function $\hat{V}$ is estimated or the action-value function $\hat{Q}$ is estimated respectively. The value functions are estimated using any of the value-based approaches discussed in previous section.

$$
\begin{aligned}
\boldsymbol{\theta}_{t+1} & =\boldsymbol{\theta}_{t}+\alpha\left(R_{t}+\gamma \hat{\mathrm{V}}\left(S_{t+1}\right)-\hat{\mathrm{V}}\left(S_{t}\right)\right) \frac{\nabla_{\boldsymbol{\theta}} \pi\left(A_{t} \mid S_{t} ; \boldsymbol{\theta}\right)}{\pi\left(A_{t} \mid S_{t} ; \boldsymbol{\theta}\right)} \\
& =\boldsymbol{\theta}_{t}+\alpha \delta_{t} \frac{\nabla_{\boldsymbol{\theta}} \pi\left(A_{t} \mid S_{t} ; \boldsymbol{\theta}\right)}{\pi\left(A_{t} \mid S_{t} ; \boldsymbol{\theta}\right)}
\end{aligned}
$$




$$
\begin{aligned}
\boldsymbol{\theta}_{t+1} & =\boldsymbol{\theta}_{t}+\alpha\left(R_{t}+\gamma \max _{a^{\prime}} \hat{\mathrm{Q}}\left(S_{t+1}, a^{\prime}\right)-\hat{\mathrm{Q}}\left(S_{t}, A_{t}\right)\right) \frac{\nabla_{\boldsymbol{\theta}} \pi\left(A_{t} \mid S_{t} ; \boldsymbol{\theta}\right)}{\pi\left(A_{t} \mid S_{t} ; \boldsymbol{\theta}\right)} \\
& =\boldsymbol{\theta}_{t}+\alpha \delta_{t} \frac{\nabla_{\boldsymbol{\theta}} \pi\left(A_{t} \mid S_{t} ; \boldsymbol{\theta}\right)}{\pi\left(A_{t} \mid S_{t} ; \boldsymbol{\theta}\right)}
\end{aligned}
$$

Deterministic Policy Gradient [32], or DPG, is an off-policy actor-critic algorithm that employs the policy gradient theorem (Theorem 2.1) and adapts it to the case of a parametrised deterministic policy $a=\pi(s ; \boldsymbol{\theta})$. The DPG algorithm can deal with continuous state and action spaces. However, in these situations, finding the action with the highest $\mathrm{Q}$-value is problematic as it requires an iterative optimisation procedure ${ }^{27}$. The DPG algorithm uses a simple alternative to that and adjust the policy parameters $\boldsymbol{\theta}$ in the direction of the gradient of the actionvalue function $\nabla_{\boldsymbol{\theta}} \hat{\mathrm{Q}}^{\pi}\left(s, \pi\left(s^{\prime} ; \boldsymbol{\theta}\right)\right)$. Because state and action spaces are continuous, the action-value function is also parametrised $\hat{\mathrm{Q}}(s, a)=\hat{\mathrm{Q}}(s, a ; \boldsymbol{w})$ where $\boldsymbol{w} \in \mathbb{R}^{g}$ is the parameter vector ${ }^{28}$.

\section{Theorem 2.2: Deterministic Policy Gradient}

Assuming an MDP in which $p\left(s^{\prime} \mid s, a\right), \nabla_{a} p\left(s^{\prime} \mid s, a\right), \pi\left(s^{\prime} ; \boldsymbol{\theta}\right), \nabla_{\boldsymbol{\theta}} \pi\left(s^{\prime} ; \boldsymbol{\theta}\right)$, $r\left(s^{\prime}, s, a\right), \nabla_{a} r\left(s^{\prime}, s, a\right)$ and $\nabla_{a} r\left(s^{\prime}, s, a\right)$ are continuous in the parameters and variables $s, a$ and $s^{\prime}$. This implies that $\nabla_{\boldsymbol{\theta}} \pi\left(s^{\prime} ; \boldsymbol{\theta}\right)$ and $\nabla_{a} \hat{\mathrm{Q}}^{\pi}(s, a ; \boldsymbol{w})$ exist and the deterministic policy gradient exists. Then:

$$
\begin{aligned}
\nabla_{\boldsymbol{\theta}} J\left(\pi_{\boldsymbol{\theta}}\right) & =\left.\int_{\mathcal{S}} \rho(s) \nabla_{\boldsymbol{\theta}} \pi\left(s^{\prime} ; \boldsymbol{\theta}\right) \nabla_{a} \hat{\mathrm{Q}}^{\pi}(s, a ; \boldsymbol{w})\right|_{a=\pi\left(s^{\prime} ; \boldsymbol{\theta}\right)} d s \\
& =\mathbb{E}_{s \sim \rho(s)}\left[\left.\nabla_{\boldsymbol{\theta}} \pi\left(s^{\prime} ; \boldsymbol{\theta}\right) \nabla_{a} \hat{\mathrm{Q}}^{\pi}(s, a ; \boldsymbol{w})\right|_{a=\pi\left(s^{\prime} ; \boldsymbol{\theta}\right)}\right]
\end{aligned}
$$

where the learning of the parametrised action-value function $\hat{\mathrm{Q}}(s, a ; \boldsymbol{w})$ is discussed in Section 2.2.3.

For the complete proof, we refer the reader to [32].

The deterministic policy gradient theorem is the limit-case of the policy gradient theorem in which the actions are chosen accordingly to a deterministic policy. Moreover, policies and value functions can be approximated with linear function approximators allowing the DPG algorithm to solve efficiently continuous state and action space problems, such as inverted pendulum stabilisation, mountain

\footnotetext{
${ }^{27}$ We need here to find the maximum of a continuous function.

${ }^{28}$ The parametrisation problem is treated in Section 2.2.3
} 
car, and continuous bandit [1].

\subsubsection{Approximating Value Functions and Policies}

So far, the theory has been presented with the underlying assumption of a finite and discrete state and action spaces. Most of the action-value functions Q estimated throughout Section 2.1 and 2.2 (with the exception of the policy-based and actorcritic methods), are none other than look-up tables of dimension $|\mathcal{S}| \times|\mathcal{A}|$, where $|\mathcal{S}|$ corresponds to the cardinality ${ }^{29}$ of the state space $\mathcal{S}$ and $|\mathcal{A}|$ to the cardinality of the action space $\mathcal{A}$. The so-called tabular approaches do not scale well if the problems grow in complexity and the state and action spaces are high-dimensional, as shown in Example 2.5. Additionally, these approaches cannot evaluate the value of states or state-action pairs that are not present in the table, i.e. tabular approaches do not generalise to unseen states or state-action pairs. Eventually, because of the lack of generalisation, these methods require a long training time as the value functions have to be individually evaluated for each state or state-action pair of the table.

\section{Example 2.5: Storing the Q-table in Memory}

Suppose we solve an MDP using tabular Q-learning, where the state space is 10-dimensional, continuous space, and the agent can choose from ten possible actions. The first step for solving this problem is to find suitable discretisation of the state space. We can partition each dimension of the state space in $x$ tiers and consequently generate $x^{10}$ possible states. In Table 2.1, we show the explosion of the size of the Q-table and the physical computer memory required in this case.

How much physical memory does a Q-table require if float numbers of type float32 are stored in it? Each real number of type float32 requires 4 bytes of memory.

\footnotetext{
${ }^{29}$ The cardinality of a set corresponds to the number of elements of the set.
} 


\begin{tabular}{||c|c|c|c|c||}
\hline Num. of tiers & Num. of states & Num. of actions & Q-table size & Memory required \\
\hline \hline 2 & $2^{10}$ & 10 & $2^{10} \times 10$ & $\approx 10 \mathrm{kB}$ \\
\hline 5 & $5^{10}$ & 10 & $5^{10} \times 10$ & $\approx 97 \mathrm{MB}$ \\
\hline 10 & $10^{10}$ & 10 & $10^{10} \times 10$ & $\approx 10 \mathrm{~GB}$ \\
\hline 100 & $100^{10}$ & 10 & $100^{10} \times 10$ & $\approx 10^{10} \mathrm{~TB}$ \\
\hline
\end{tabular}

Table 2.1: Memory required to store a Q-table for different discretisation tiers of the state space.

Only in MDPs with small state and action spaces, it is possible to apply tabular approaches.

In many interesting real-world problems, especially in robotics, the state and the action spaces are high-dimensional and continuous spaces that make impossible the use of tabular approaches described so far for estimating the value functions.

Similarly to the case of the policy parametrisation in Equation (2.24), we can parametrise the value function with a parameter vector $\boldsymbol{w} \in \mathbb{R}^{g}$ and we denote it with $\hat{\mathrm{V}}(s, \boldsymbol{w})$. Independently of the choice of the parametrisation, we can update the parameters $\boldsymbol{w}$ at each time step with (stochastic) gradient descent using the samples obtained at time step $t$ as:

$$
\begin{aligned}
\boldsymbol{w}_{t+1} & =\boldsymbol{w}_{t}-\frac{1}{2} \alpha \nabla_{\boldsymbol{w}}\left[\mathrm{V}^{\pi}\left(S_{t}\right)-\hat{\mathrm{V}}\left(S_{t} ; \boldsymbol{w}_{t}\right)\right]^{2} \\
& =\boldsymbol{w}_{t}+\alpha\left[\mathrm{V}^{\pi}\left(S_{t}\right)-\hat{\mathrm{V}}\left(S_{t} ; \boldsymbol{w}_{t}\right)\right] \nabla_{\boldsymbol{w}} \hat{\mathrm{V}}\left(S_{t} ; \boldsymbol{w}_{t}\right)
\end{aligned}
$$

where the parameter vector $\boldsymbol{w}$ is updated by minimising the prediction error between the true value of the state $\mathrm{V}^{\pi}\left(S_{t}\right)$ and the predicted using the parametrised value function $\hat{\mathrm{V}}\left(S_{t}, \boldsymbol{w}_{t}\right)$. By minimising the prediction error, the estimate of the value function tends to the true value function. Similarly to the policy parametrisation, the function parametrising the value function must be differentiable with respect to its parameters $\boldsymbol{w}$.

Equation (2.37) assumes that the true value function $\mathrm{V}^{\pi}\left(S_{t}\right)$ is known before hand and can be used to generate a fixed target value. However, in practice we never know the value function a priori, thus we need to find a valid replacement to $\mathrm{V}^{\pi}\left(S_{t}\right)$. Similarly to what we have seen in Equation (2.18 and 2.20), driven by the Bellman Equation, we can approximate the true value function $\mathrm{V}^{\pi}\left(S_{t}\right)$ with 
the episode return $\mathrm{G}_{t}$ (see Equation (2.38)) or with the TD-error (see Equation $(2.39))$.

$$
\begin{gathered}
\boldsymbol{w}_{t+1}=\boldsymbol{w}_{t}-\frac{1}{2} \alpha \nabla_{\boldsymbol{w}}\left[\mathrm{G}_{t}-\hat{\mathrm{V}}\left(S_{t} ; \boldsymbol{w}_{t}\right)\right]^{2} \\
=\boldsymbol{w}_{t}+\alpha\left[\mathrm{G}_{t}-\hat{\mathrm{V}}\left(S_{t} ; \boldsymbol{w}_{t}\right)\right] \nabla_{\boldsymbol{w}} \hat{\mathrm{V}}\left(S_{t} ; \boldsymbol{w}_{t}\right) \\
\boldsymbol{w}_{t+1}=\boldsymbol{w}_{t}-\frac{1}{2} \alpha \nabla_{\boldsymbol{w}}\left[R_{t}+\gamma \hat{\mathrm{V}}\left(S_{t+1} ; \boldsymbol{w}_{t}\right)-\hat{\mathrm{V}}\left(S_{t} ; \boldsymbol{w}_{t}\right)\right]^{2} \\
=\boldsymbol{w}_{t}+\alpha\left[R_{t}+\gamma \hat{\mathrm{V}}\left(S_{t+1} ; \boldsymbol{w}_{t}\right)-\hat{\mathrm{V}}\left(S_{t} ; \boldsymbol{w}_{t}\right)\right] \nabla_{\boldsymbol{w}} \hat{\mathrm{V}}\left(S_{t} ; \boldsymbol{w}_{t}\right)
\end{gathered}
$$

The same approach can be used to approximate the action-value function $\hat{\mathrm{Q}}(s, a ; \boldsymbol{w})$. For example, we show, in Equation $(2.40)$, the update rule of the parameter vector $\boldsymbol{w}$ when the TD-error is used in off-policy settings, since it is the core principle of the algorithms in Chapter 3.

$$
\begin{aligned}
\boldsymbol{w}_{t+1} & =\boldsymbol{w}_{t}-\frac{1}{2} \alpha \nabla_{\boldsymbol{w}}\left[R_{t}+\gamma \max _{a} \hat{\mathrm{Q}}\left(S_{t+1}, a ; \boldsymbol{w}_{t}\right)-\hat{\mathrm{Q}}\left(S_{t}, A_{t} ; \boldsymbol{w}_{t}\right)\right]^{2} \\
& =\boldsymbol{w}_{t}+\alpha\left[R_{t}+\gamma \max _{a} \hat{\mathrm{Q}}\left(S_{t+1}, a ; \boldsymbol{w}_{t}\right)-\hat{\mathrm{Q}}\left(S_{t}, A_{t} ; \boldsymbol{w}_{t}\right)\right] \nabla_{\boldsymbol{w}} \hat{\mathrm{V}}\left(S_{t}, A_{t} ; \boldsymbol{w}_{t}\right)
\end{aligned}
$$

Many function approximators exist and can be used to approximate value functions and policies. However, here we focus on artificial neural networks [33], the most widely-used non-linear function approximators in Deep Learning [34]. When the artificial neural network is composed of multiple layers, we usually referred to it as a deep neural network (see Section 3.1.4). When deep neural networks are used in Reinforcement Learning, we usually speak of Deep Reinforcement Learning. 


\section{Chapter 3}

\section{Deep Reinforcement Learning}

In this chapter, we focus on the use of artificial neural networks, or ANNs, as function approximators for value functions and policies. Firstly, a concise introduction to ANNs is presented. Secondly, the Deep Reinforcement Learning algorithms used in this thesis are introduced, followed by the discussion on the problem of state representation learning for Reinforcement Learning. Eventually, the challenges of Reinforcement Learning in robotics are explained and discussed. 


\subsection{Artificial Neural Networks}

\subsubsection{Feedforward Neural Networks}

Feedforward neural networks, or multi-layer perceptrons, [34] lay the foundations of Deep Learning. Feedforward neural networks are a family of parametric models used to approximate any unknown function $f: \mathcal{X} \longrightarrow \mathcal{Y} ; x \longmapsto f(x)$, mapping an input $x$ with dimensionality $n$ to an output $y=f(x)$ with dimensionality $m$, by means of a parametric function $\hat{f}$ such that $y=\hat{f}(x ; \boldsymbol{w})$. The neural network parameters $\boldsymbol{w} \in \mathbb{R}^{\omega}$ are optimised, via a training set of examples and gradient descent, to find the best approximation of $f$. An example of a two layers feedforward neural network is shown in Figure 3.1.

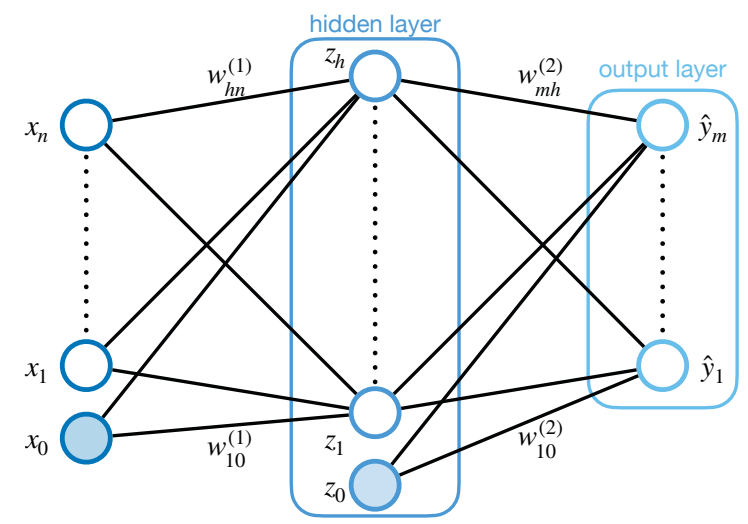

Figure 3.1: Two-layers feedforward neural networks with $n$ inputs, $h$ hidden neurons and $m$ outputs. The input and output are indicated with $x$, and $y$, the weights of the network are indicated with $w$ (e.g. $w_{h n}^{(1)}$ corresponds to the weight of the first layer connecting $n^{t h}$-input to the $h^{t h}$-hidden node) and the biases with the subscript 0 .

Assuming the feedforward network depicted in Figure 3.1 with $n$ inputs, $h$ hidden neurons, or nodes, $m$ outputs, a set weights and biases $\boldsymbol{w}$, i.e. the parameters, and an activation function $\xi(\cdot)$, we can define the contribution of the 
$i^{t h}$-component of the input $x_{i}$ to the $k^{t h}$-component of the output $y_{k}$ as:

$$
\begin{aligned}
& a_{j}=\sum_{i=1}^{n} w_{j i}^{(1)} x_{i}+w_{j 0}^{(1)} \\
& z_{j}=\xi\left(a_{j}\right) \\
& a_{k}=\sum_{j=1}^{h} w_{k j}^{(2)} z_{j}+w_{k 0}^{(2)} \\
& y_{k}=\xi\left(a_{k}\right)
\end{aligned}
$$

If the activation function $\xi(\cdot)$ is chosen linear, the output is a linear combination of the inputs. However, in most cases, the activation function is chosen non-linear. Feedforward neural networks with non-linear activations are universal function approximators [35]. Common activation functions are sigmoid, tanh, rectified linear unit, or ReLU, and softmax. Usually the activations of all the hidden layers are chosen to be the same and only the activations of the output layer differ in relation to the type of output required, e.g. a probability value $\in[0,1]$ or a bounded value $\in[-1,1]$.

These parametric models are called networks because they employ layer-wise compositions of different functions, e.g. $y=\hat{f}(x)=\hat{f}^{(2)}\left(\hat{f}^{(1)}(x)\right)$ with $\hat{f}^{(1)}(x)=$ $\sum_{j=1}^{h} \xi\left(\sum_{i=1}^{n} w_{j i}^{(1)} x_{i}+w_{j 0}^{(1)}\right)$ and $\hat{f}^{(2)}(z)=\sum_{k=1}^{m} \xi\left(\sum_{j=1}^{h} w_{k j}^{(2)} z_{j}+w_{k 0}^{(2)}\right)$. The amount of layers $\left(\hat{f}^{(1)}, \hat{f}^{(2)}\right.$, etc) defines the depth of the neural network, while number of neurons in each layer its width. If each neuron of a given layer is connected to all the neurons of the successor layer, we call this type of layers fully-connected or dense. The term neural, coming from neuroscience, is employed because these networks are inspired by the functioning of human brains, where each biological neuron receives signals from neighbouring neurons, computes its activation and produces an output signal than is then broadcasted to adjacent neurons. Eventually, the term feedforward is used because the input-output mapping contains no form of signal coming from the output to the input.

Independently of the structure of the network, we need a way to learn the parameters $\boldsymbol{w}$, i.e. weights and biases, of the network to best approximate the target and unknown function $f$, given a set of samples. We can do this by means of gradient descent and back-propagation by choosing a suitable loss, or cost function $\mathcal{L}$. From now on, we indicate the loss function as $\mathcal{L}(\boldsymbol{w})$ to explicitly highlight the 
dependency of the loss on the parameters' vector $\boldsymbol{w}$. In particular, in Supervised Learning settings ${ }^{1}$ given a training set of input data $x^{(1)}, \ldots, x^{(l)}$ and target output data $y^{(1)}, \ldots, y^{(l)}$ and the set of predicted outputs $\hat{y}^{(1)}, \ldots, \hat{y}^{(l)}$, where $\hat{y}^{(i)}=$ $\hat{f}\left(x^{(i)} ; \boldsymbol{w}\right)$, we can compute the prediction errors of the neural network $\hat{f}$ and adjust the network parameters to minimise such errors. For example, a commonly used loss function is the mean square error (MSE) loss, over the training set, as shown in Equation (3.2).

$$
\begin{array}{rl}
\min _{\boldsymbol{w}} & \mathcal{L}(\boldsymbol{w}) \\
\mathcal{L}(\boldsymbol{w})= & \frac{1}{l} \sum_{i=1}^{l}\left\|y^{(i)}-\hat{f}\left(x^{(i)} ; \boldsymbol{w}\right)\right\|^{2} \approx \mathbb{E}_{x, y \sim \hat{p}_{\text {data }}}\|y-\hat{f}(x ; \boldsymbol{w})\|^{2}
\end{array}
$$

where $l$ is the number of data in the training set, $y^{(i)}$ is the $i$-th target output data, $\hat{f}\left(x^{(i)} ; \boldsymbol{w}\right)$ is the predicted output given the input $x^{(i)}$, and we assume the input and output data, $x^{(1)}, \ldots, x^{(l)}$ and $y^{(1)}, \ldots, y^{(l)}$ respectively, to be sampled from the empirical distribution of the training set $\hat{p}_{\text {data }}{ }^{2}$. At each training operation, we compute the gradient $\nabla_{\boldsymbol{w}} \mathcal{L}(\boldsymbol{w})$ with respect to the network parameters and then back-propagate this gradient through each layer and neuron from output to input. Computing the expectation over the whole training dataset $\mathbb{E}_{x, y \sim \hat{p}_{\text {data }}}$ is usually expensive in terms of computations and hardware resources, thus usually a random subset of data, i.e. a minibatch ${ }^{3}$, is randomly sampled and used to train the neural networks. For more details on back-propagation and optimisation methods for training deep neural networks, we refer the reader to [34] and [36].

\subsubsection{Convolutional Neural Networks}

Convolutional neural networks [37], or CNN, are neural networks specialised in efficiently handling data with grid-like structure, e.g. RGB images (2D pixel grids) or LiDAR readings (1D data grids). Convolutional neural networks inherit their name from the mathematical operator that they employ in at least one of their layers, i.e. the convolution.

\footnotetext{
${ }^{1}$ In Supervised Learning the set of output data is labelled by an expert, e.g. in classification problems, in Unsupervised Learning no expert data is provided and we seek to cluster our input data. In Reinforcement Learning we do not have any target and we are interested in finding the best actions to achieve a given goal.

${ }^{2}$ For more information on the data distribution, we refer the reader to [34].

${ }^{3}$ The size of the minibatch $b$ is usually way smaller than the size of the training set $l$.
} 
For example, given a sequence of 1D noisy sensor reading measuring the position of a robot over time $x(t)$ with $t \in(-\infty, \infty)^{4}$, we would like to obtain a better estimate of the robot position $x(t)$ by averaging multiple measurements over time. However, the contribution of past measurements should be dependent on the time instant the measurements are obtained, and older measurements should be trusted less than the newer ones. Therefore, to find a smooth estimate of the robot pose, we need a weighting function $\omega(a)$ dependent on the age $a$ of the measurements we need to apply for each time instant $t$. The function $\omega$ is usually called kernel or filter. This whole operation is called convolution and is indicated by $*$. If $t$ is a continuous variable, the smoothed position of the robot $s(t)$ can be expressed as:

$$
s(t)=(x * w)(t)=\int_{-\infty}^{t} x(a) \omega(t-a) d a
$$

In general, we deal with computers and sensors providing data at discrete time steps, thus we can replace the integral from Equation (3.3) with the sum over all time steps:

$$
s(t)=(x * w)(t)=\sum_{a=-\infty}^{t} x(a) \omega(t-a)
$$

We can also use the convolution operator over multi-dimensional inputs, but in particular, we are interested in using convolutions for the special case of $2 \mathrm{D}$ inputs, e.g. images. Given a 2D image $I$ of size $m \times n$ and a $2 \mathrm{D}$ kernel $K$, the convolution operation of sliding the kernel over the image can be defined as:

$$
S(i, j)=(I * K)(i, j)=\sum_{i=1}^{m} \sum_{j=1}^{n} I(m, n) K(i-m, j-n)
$$

After defining the convolution operator, we want to understand how it is used in the convolutional neural networks and what are the advantages with respect to feedforward neural networks. First, differently from feedforward networks that use full connectivity of each input and output neuron, convolutional networks are an example of sparse connectivity when the kernel size is smaller than the input data $\operatorname{size}^{5}$. Convolution allows reducing the number of network parameters, the physical

\footnotetext{
${ }^{4}$ In principle, the time variable can be either continuous or discrete, but in practice, it is always discrete due to the use of digital computers and sensors.

${ }^{5}$ For example, images can contain hundreds or millions of pixels, while the kernels have way smaller dimensions. Kernels are used because we are interested in learning to extract meaningful
} 
memory required to store it and the number of computations for computing the outputs.

In a convolutional layer of a neural network, we can identify three main operations:

- apply convolution to generate activations (see Figure 3.2)

- pass the activations through non-linear activation functions (e.g. ReLU)

- apply pooling to change (reduce) the shape of the output of the layer ${ }^{6}$

The trainable parameters of convolutional neural networks are the elements of the kernels. These parameters can be learned with the same approach used by feedforward neural networks by defining a loss function and adjusting the network parameters according to its gradient with respect to the parameters, i.e. via backpropagation. By learning the weights of the kernels, it is possible to identify different features of the input data, for example, edges and shapes in the case of 2D images. Convolutional neural networks employ parameter sharing to reduce the number of parameters with respect to feedforward neural networks. Multiple kernels are slid with a certain stride length ${ }^{7}$ across the whole data grid to extract different features. Figure 3.2 shows an example of convolution applied to an image. As in the case of feedforward networks, multiple convolutional layers can be stacked together. The more the convolutional layer is close to the network's output, the higher is the level of abstraction the learned features (e.g. from edges and colours to more complex shapes).

The most widely-studied example of the use of CNNs is probably the problem of classifying images. Given a training set of labelled images ${ }^{8}$ (e.g. a data set containing images of dogs and cats and the correct labels), the neural network has to learn to properly determine if the input images belong to one of the data classes. Therefore, the network has to learn to extract the representative features of each class to distinguish among them.

features in the images, such as edges or geometrical shapes, which generally occupy multiple pixels.

${ }^{6}$ A common type of pooling operation, called max-pooling, outputs only the maximum value of a rectangular neighbourhood. Pooling allows to progressively reduce the dimensionality of the input data layer after layer.

${ }^{7}$ The stride is the parameter determining how much the kernel is shifted.

${ }^{8}$ The learning approach requiring manual labelling of the training data is called Supervised Learning. 


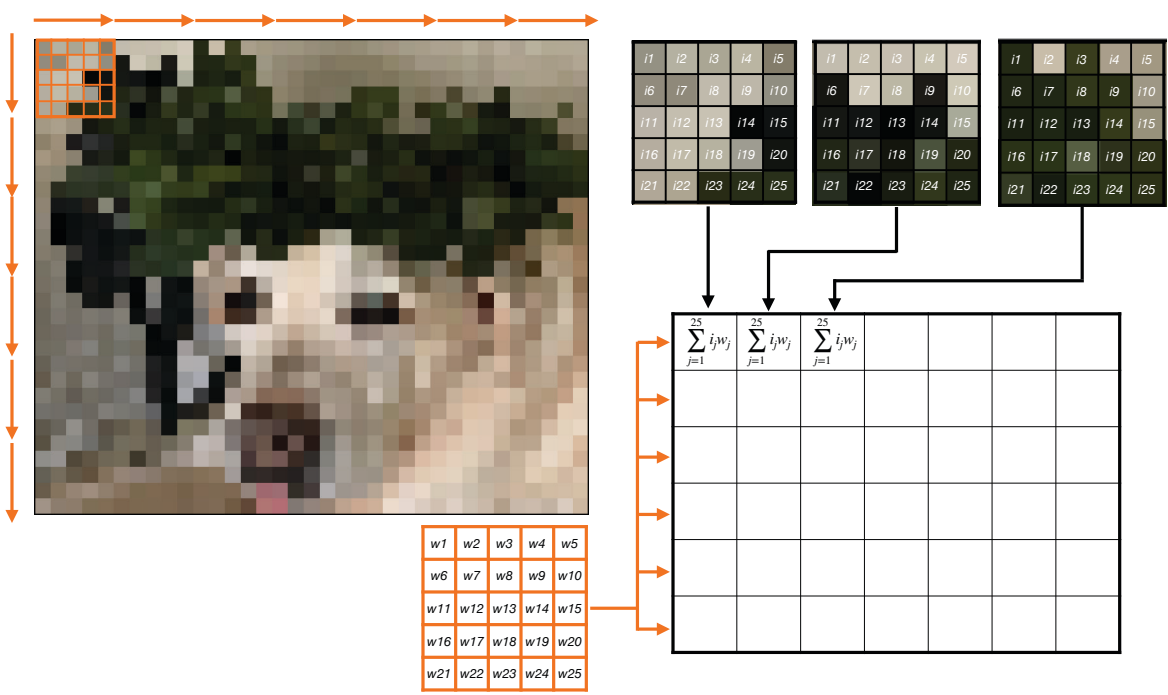

Figure 3.2: Example of 2D-convolution with kernel with size $5 \times 5$ and stride 5. Original image can be found at: https://drive.google.com/file/d/ 1GEjmhLB5T-kEcHtQMYsdIKrDKmD8fVaV/view?usp=sharing.

\subsubsection{Recurrent Neural Networks}

Recurrent neural networks [38], or RNNs, are the class of neural networks specialised in handling sequences of data with either fixed or variable length. RNNs can be used in different contexts, for example, to map sequences to sequences (e.g. natural language processing), sequences to a single output (e.g. partially observable environment in Reinforcement Learning).

Similar to the case of feedforward networks, we can analytically define the relation between the input vector $x$, the hidden state vector $h$ and the predicted output $\hat{y}$ as:

$$
\begin{aligned}
& a_{t}=W h_{t-1}+U x_{t}+b \\
& h_{t}=\xi\left(a_{t}\right) \\
& \hat{y}_{t}=V h_{t}+c
\end{aligned}
$$

where $W, U, V$ are the weight matrices and $b, c$ are the bias vectors.

RNNs, as feedforward NNs and CNNs, are trained by defining a loss function, either using target labelled data, as in the case of Supervised Learning or not, 

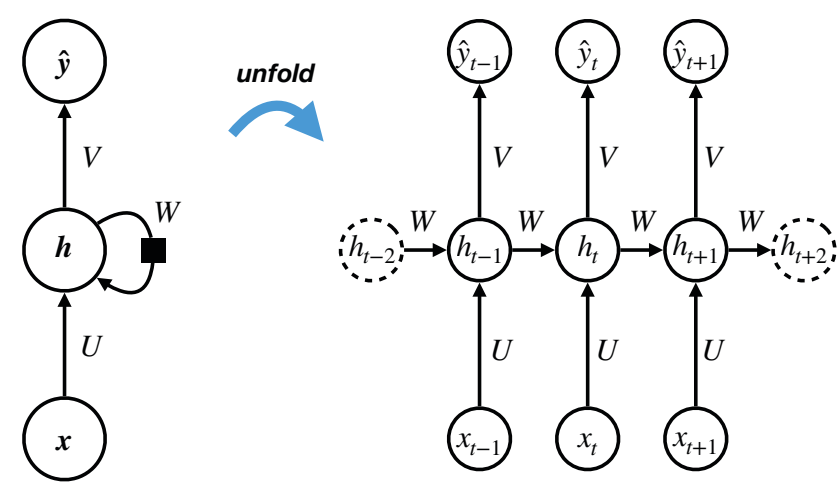

Figure 3.3: Computational graph of a recurrent neural network.

and by applying back-propagation. For more information on RNNs, we refer the readers to [34] and [39].

\subsubsection{Deep Neural Networks}

Feedforward, convolutional, and recurrent layers can be and are usually stacked together to improve the expressivity of the overall neural networks. When the neural network model is composed of multiple layers, we usually referred to it as a deep neural network.

Deep neural networks allow to reduce and often cancel out the need for tedious data pre-processing and features engineering. For example, in Supervised Learning, the deep neural network learns to extract features and learns to classify the data using the learned features.

\subsection{Reinforcement Learning with Deep Neural Net- works}

In Chapter 2 (Section 2.2.3) we have discussed the necessity and the advantages of using function approximators in Reinforcement Learning. When deep neural networks are used in Reinforcement Learning for approximating value functions and policies from raw data without hand-crafting the state vector, we usually 
speak of Deep Reinforcement Learning. In this section, we introduce and discuss the algorithms used in later chapters of this dissertation.

The Reinforcement Learning principles, described in Chapter 2, still hold for the case of Deep Reinforcement Learning. However, despite their great success, neural networks bring into Reinforcement Learning several drawbacks such as training instabilities ${ }^{9}$ and no, or limited, convergence guarantees.

In particular, when training neural networks, except in the specific case of recurrent networks, we assume that each data point (tuple) is sampled from an independent and identically distribution. This assumption is often referred to in the literature as the i.i.d. hypothesis. Unfortunately, this is never the case in Reinforcement Learning, where each sample comes from a temporally correlated sequence of states, actions and rewards. Because each state is temporally correlated to its predecessors and successors, the i.i.d. hypothesis does not hold anymore!

To alleviate the problem of the training instability brought by the temporal correlation of the samples, all the experience collected is stored in a memory buffer $\mathcal{D}$, i.e. a container of all, or most, of the experience tuples obtained by interacting with the environment. When training the neural networks, we can randomly sample mini-batches of data ${ }^{10}$ and we can reduce the training instability of the networks. Moreover, thanks to the memory buffer, the interaction samples can be used repeatedly, for example, to find the best fitting value function or improve the policy performances. While the update of the neural networks can be done using gradient ascent, for networks representing policies, and to the gradient descent, for networks representing value functions, as shown in Equation (2.26) and (2.37)-(2.39), this operation is not sample-efficient as, in the case of the methods presented in Chapter 2, we use only the samples at the current time step to update the networks and immediately discard them after. For these two reasons, Deep Reinforcement Learning algorithms use a memory buffer to store all the experience samples collected during the interaction with the environment.

\footnotetext{
${ }^{9}$ Common instabilities are divergence and oscillations of the approximated policies or value functions from and around the optimal values or convergence to local minima.

${ }^{10}$ By randomly sampling the experience tuples, we break their temporal correlation.
} 


\subsubsection{Deep Q-Network}

Deep Q-Network [40], or DQN, is one of the first successful examples of a Deep Reinforcement Learning algorithm. DQN is the stable extension of Q-learning to deep neural networks. The DQN agents can learn optimal action-value functions and derive optimal policies directly from high-dimensional raw input data, e.g. RGB images, without any need of a hand-crafted and low-dimensional state vector, even in continuous state spaces, e.g. robot pose in the $2 \mathrm{D}$ or $3 \mathrm{D}$ space. The DQN agent, proposed in [40], could play seven different Atari games with performances comparable to humans. A few years later, DQN was further improved [41] to play all the Atari games with performances superior to human players in most of the games. The three main elements of the outstanding success of DQN [41] are:

- approximation of the action-value function Q using a neural network

- experience replay buffer to break temporal correlation of the samples

- target network to generate a fixed Temporal Difference-target ${ }^{11}$ and increase the training stability

In DQN, the action-value function Q, differently from the tabular approach used by Q-learning, is approximated by a neural network, as shown in Figure 3.4. In the simplest case, the neural network directly replaces the look-up table used in Q-learning that inputs a state $s$ and an action $a$ and outputs the Q-value of the state-action pair $\hat{\mathrm{Q}}(s, a ; \boldsymbol{w})$, where $\boldsymbol{w}$ is the parameter vector of the neural network. However, usually a more computationally efficient approach is used and only the state is fed to the neural network and the action-value function of all the possible actions $\hat{\mathrm{Q}}\left(s, a_{1} ; \boldsymbol{w}\right), \ldots, \hat{\mathrm{Q}}\left(s, a_{n} ; \boldsymbol{w}\right)$ is estimated.

The DQN algorithm makes use of a memory buffer to store the experience samples ${ }^{12}$ and, consequently, train the network approximating the action-value function using randomly sampled mini-batches from the memory buffer. This mechanism is called experience replay. We can then update the parameters $\boldsymbol{w}$ of the neural network as a simple supervised learning problem by minimising the mean square error loss function between the true value function and the prediction

\footnotetext{
${ }^{11}$ From now on, we will refer to it as TD-target.

${ }^{12}$ The memory buffer contains a considerable yet limited number of samples, and it is usually implemented as a First-In-First-Out queue. Therefore, old samples are gradually replaced with the newest ones.
} 

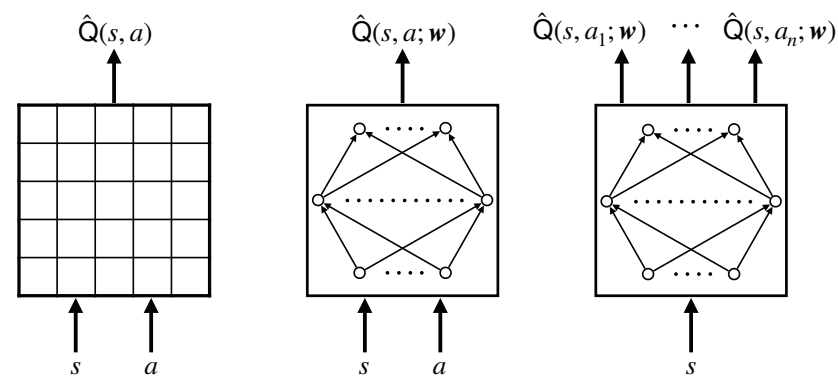

Figure 3.4: Different ways of representing and estimating the action-value function Q. On the left the tabular approach of Q-learning, where the Q is none other than a look-up table. In the middle and on the right, the $\mathrm{Q}$ function is approximated by a neural network.

of the network, as shown in Equation (3.7).

$$
\begin{array}{rl}
\min _{\boldsymbol{w}} & \mathcal{L}(\boldsymbol{w}) \\
\mathcal{L}(\boldsymbol{w})= & \mathbb{E}_{s, a \sim \mathcal{D}}\left[\|\mathrm{Q}(s, a)-\hat{\mathrm{Q}}(s, a ; \boldsymbol{w})\|^{2}\right]
\end{array}
$$

As shown in Section 2.2.3, we do not normally know the true Q-value of a stateaction pair, thus we employ an approximation by means of TD-error ${ }^{13}$. Thus the loss function in Equation (3.7) can be rewritten as:

$$
\mathcal{L}(\boldsymbol{w})=\mathbb{E}_{s, a, r, s^{\prime} \sim \mathcal{D}}\left[\left\|r+\gamma \max _{a^{\prime}} \hat{\mathrm{Q}}^{-}\left(s^{\prime}, a^{\prime} ; \boldsymbol{w}^{-}\right)-\hat{\mathrm{Q}}(s, a ; \boldsymbol{w})\right\|^{2}\right]
$$

where $\hat{\mathrm{Q}}^{-}$is the so-called target network used to compute the TD-target $r+$ $\gamma \max _{a^{\prime}} \hat{\mathrm{Q}}^{-}\left(s^{\prime}, a^{\prime} ; \boldsymbol{w}^{-}\right)$. The target network $\hat{\mathrm{Q}}^{-}\left(s^{\prime}, a^{\prime} ; \boldsymbol{w}^{-}\right)$is an exact copy of the Q-network, but with parameters frozen at an arbitrary previous training iteration, i.e. the gradient of the loss function does not flow through the target network. Using a fixed TD-target greatly improves the stability of the training because the network that is updated chase a fixed target rather that a continuously changing

\footnotetext{
${ }^{13}$ This is the exact same procedure used by Q-learning, see Equation (2.21) and shown in Equation 2.40
} 
one ${ }^{14}$.

The complete Deep Q-network algorithm [41] is presented in Algorithm 6.

\section{Pseudo-code 3.1: Deep Q-Network with Experience Replay}

Algorithm 6: Adapted from [41]

Initialise the replay buffer $\mathcal{D}$ with capacity $\mathrm{D}$;

Initialise the action-value function network $\hat{Q}$ with random weights $\boldsymbol{w}$;

Initialise the target action-value function network $\hat{\mathrm{Q}}^{-}$with random

weights $\boldsymbol{w}^{-}=\boldsymbol{w}$;

for episode $=0, M-1$ do

Set episode initial state $S_{0}=s_{\text {init }}$;

for $t=0, N-1$ do

With probability $\epsilon$ select a random action $A_{t}$;

otherwise select $A_{t}=\arg \max _{a} \hat{\mathrm{Q}}\left(S_{t}, a_{t} ; \boldsymbol{w}\right)$;

Execute action $A_{t}$ and observe reward $R_{t}$ and next state $S_{t+1}$;

Store transition $\left(S_{t}, A_{t}, R_{t}, S_{t+1}\right)$ in $\mathcal{D}$;

Sample random mini-batch of transitions $\left(S_{j}, A_{j}, R_{j}, S_{j+1}\right)$ from $\mathcal{D}$;

Compute target

$$
y_{j}= \begin{cases}R_{j}, & \text { for terminal } S_{j} \\ R_{j}+\gamma \max _{a^{\prime}} \hat{\mathrm{Q}}^{-}\left(S_{j+1}, a_{j+1} ; \boldsymbol{w}^{-}\right), & \text {otherwise }\end{cases}
$$

Perform a gradient descent step on $\mathcal{L}(\boldsymbol{w})=\left(y_{j}-\hat{\mathrm{Q}}\left(S_{j}, A_{j} ; \boldsymbol{w}\right)\right)^{2}$

with respect to the network parameters $\boldsymbol{w}$;

Update every $C$ steps the target network $\hat{\mathrm{Q}}^{-}=\hat{\mathrm{Q}}$;

end

end

\subsubsection{Deep Deterministic Policy Gradient}

So far, we have mainly shown algorithms where the agent can only select actions from a discrete action set. However, in many cases, especially in robotics control tasks (e.g. inverted pendulum stabilisation, control of a multi-joint robot arm), the problems have a continuous state space and a continuous and high-dimensional action space.

\footnotetext{
${ }^{14}$ This would happen if also the target would be affected by the gradient flow and not be fixed.
} 
Deep Deterministic Policy Gradient [42], or DDPG, can be seen as the extension of $\mathrm{DQN}^{15}$ to continuous action spaces when no explicit discretisation of the action space is employed ${ }^{16}$. The DDPG algorithm also extends the DPG algorithm (see Chapter 2, Section 2.2.2) to non-linear function approximators, i.e. neural networks. As DPG, DDPG is an actor-critic algorithm that explicitly optimises the policy $\pi$, or actor, with parameters' vector $\boldsymbol{\theta}^{\pi}$, and estimates the action-value function $\hat{Q}$, or critic, with parameters' vector $\boldsymbol{\theta}_{\hat{\mathrm{Q}}}$. Similarly to DQN, DDPG uses experience replay and target networks, with parameters' vectors $\boldsymbol{\theta}_{\pi^{-}}$ and $\boldsymbol{\theta}_{\hat{\mathrm{Q}}^{-}}$, to stabilise the training of the neural networks. However, the update of target networks is performed differently from DQN, where after a fixed amount of steps, the target network is suddenly updated. In DDPG, the target networks are slowly and smoothly updated using a weighted sum, governed by the parameter $\tau$, of the critic and actor parameters $\boldsymbol{\theta}_{\hat{\mathrm{Q}}}$ and $\boldsymbol{\theta}_{\pi}$, and the target-critic and target-actor parameters $\boldsymbol{\theta}_{\hat{\mathrm{Q}}^{-}}$and $\boldsymbol{\theta}_{\pi^{-}}$, as shown in Equation (3.9).

$$
\begin{aligned}
& \boldsymbol{\theta}_{\hat{\mathrm{Q}}^{-}} \longleftarrow \tau \boldsymbol{\theta}_{\hat{\mathrm{Q}}}+(1-\tau) \boldsymbol{\theta}_{\hat{\mathrm{Q}}^{-}} \\
& \boldsymbol{\theta}_{\pi^{-}} \longleftarrow \tau \boldsymbol{\theta}_{\pi}+(1-\tau) \boldsymbol{\theta}_{\pi^{-}}
\end{aligned}
$$

The critic network is estimated with the same procedure used by DQN by minimising the mean square error between the TD-target and the critic network prediction (see Equation (3.7) and (3.8)). However, the TD-target is not evaluated with the greedy maximisation that is not feasible for continuous action spaces, but by simply predicting the value of the next state and next action, chosen accordingly to the target policy $\pi^{-}\left(s ; \boldsymbol{\theta}_{\pi^{-}}\right)$. The actor network is instead updated using the deterministic policy gradient theorem as in the DPG case.

DDPG uses a stochastic policy for exploration obtained by adding noise $\mathcal{O}$ to the deterministic action selected by the policy $\pi(s ; \boldsymbol{\theta})$, as shown in Equation (3.10).

$$
a=\pi(s ; \boldsymbol{\theta})+\mathcal{O}
$$

The noise $\mathcal{O}$ can be any random process, but usually, an Ornstein-Uhlenbeck

\footnotetext{
${ }^{15} \mathrm{~A}$ DQN agent selects at each time step the action that maximises the Q-value, which is computationally expensive if the action space is continuous and requires an iterative optimisation procedure.

${ }^{16}$ It is always possible to more finely discretised the action space at the price of the exponential increment of the number of actions.
} 
process [43], or OU, is used to generate temporally-correlated exploration, which is suitable for many physical control problems [42].

The complete DDPG algorithm is shown in Algorithm 7.

\section{Pseudo-code 3.2: Deep Deterministic Policy Gradient}

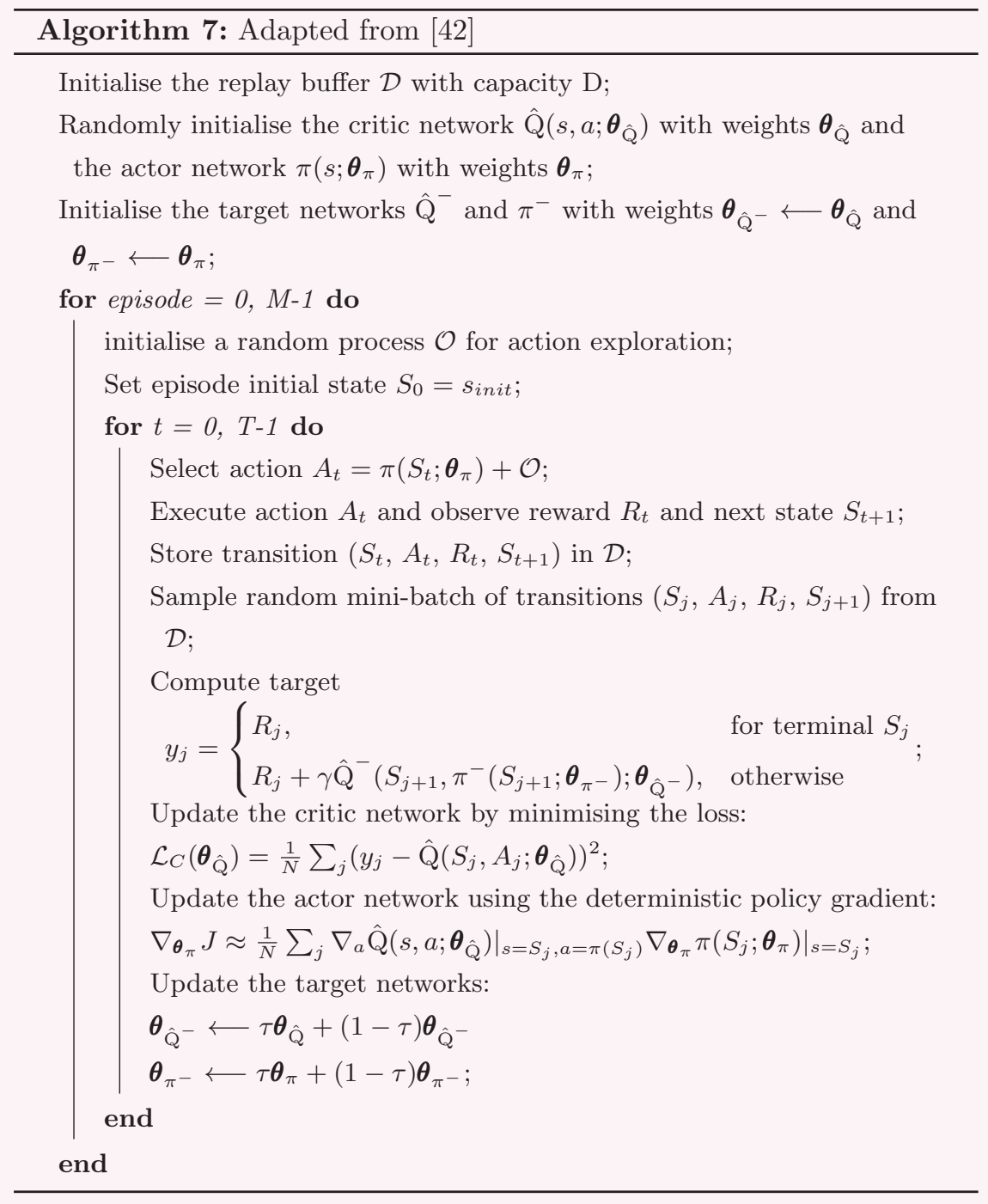




\subsubsection{Twin Delayed Deep Deterministic Policy Gradient}

Twin Delayed Deep Deterministic Policy Gradient [44], or TD3, is an actor-critic algorithm addressing the problem of overestimation of the action-value function of DDPG by employing a second critic network ${ }^{17}$.

TD3 utilises two critic networks to estimate $\hat{\mathrm{Q}}_{1}\left(s, a ; \boldsymbol{\theta}_{\hat{\mathrm{Q}}_{1}}\right)$ and $\hat{\mathrm{Q}}_{2}\left(s, a ; \boldsymbol{\theta}_{\hat{\mathrm{Q}}_{2}}\right)$, parametrised by $\boldsymbol{\theta}_{\hat{\mathrm{Q}}_{1}}$ and $\boldsymbol{\theta}_{\hat{\mathrm{Q}}_{2}}$ respectively, and an actor neural network $\pi\left(s ; \boldsymbol{\theta}_{\pi}\right)$ to approximate a continuous policy, parametrised by $\boldsymbol{\theta}_{\pi}$. The policy $\pi_{\boldsymbol{\theta}_{\pi}}: \mathcal{S} \longrightarrow \mathcal{A}$ is a deterministic policy mapping states to actions, but to guarantee sufficient exploration during the training phase, noise $\epsilon \sim \mathcal{N}(0, \sigma)$, from a Normal distribution $\mathcal{N}(0, \sigma)$ with zero mean and standard deviation $\sigma$, is added to the action $a \sim \pi\left(s ; \boldsymbol{\theta}_{\pi}\right)+\epsilon$. As in any other Deep Reinforcement Learning algorithm, the experience tuples $\left(s, a, s^{\prime}, r\right)$, collected through the interaction with the environment, are stored in the memory buffer and used to update the neural network.

Similarly to DDPG, TD3 uses target networks for critics $\hat{\mathrm{Q}}_{1}^{-}, \hat{\mathrm{Q}}_{2}^{-}$and actor $\pi^{-}$with parameters $\boldsymbol{\theta}_{\hat{\mathrm{Q}}_{1}^{-}}, \boldsymbol{\theta}_{\hat{\mathrm{Q}}_{2}^{-}}$, and $\boldsymbol{\theta}_{\pi^{-}}$respectively.

While in DDPG, the Temporal Difference-error, or TD-error, $y$ is computed using the single target critic network (see Equation (3.11)), TD3 makes use of both critic networks to reduce the overestimation generated by the use of a single value function, as can be seen in Equation (3.12).

$$
\begin{gathered}
y=r+\gamma \hat{\mathrm{Q}}^{-}\left(s^{\prime}, a ; \boldsymbol{\theta}_{\hat{\mathrm{Q}}^{-}}\right) \\
y=r+\gamma \min _{i=1,2} \hat{\mathrm{Q}}_{i}^{-}\left(s^{\prime}, \tilde{a} ; \boldsymbol{\theta}_{\hat{\mathrm{Q}}_{i}^{-}}\right)
\end{gathered}
$$

where $\tilde{a} \sim \pi^{-}\left(s ; \boldsymbol{\theta}_{\pi^{-}}\right)+\epsilon$, and $\epsilon \sim \operatorname{clip}(\mathcal{N}(0, \tilde{\sigma}),-c, c), c$ a constant hyperparameter of the algorithm. After computing the TD-error, the parameters of critic networks are updated accordingly to Equation (3.13).

$$
\boldsymbol{\theta}_{\hat{\mathrm{Q}}_{i}} \longleftarrow \min _{\boldsymbol{\theta}_{\hat{\mathrm{Q}}_{i}}} \frac{1}{N} \sum\left(y-\hat{\mathrm{Q}}_{i}(s, a)\right)^{2}
$$

As in DDPG, the actor is updated using the deterministic policy gradient theorem and the gradient of the critic $\nabla_{a} \hat{\mathrm{Q}}_{1}(s, a)$ as shown in Equation (3.14). However,

\footnotetext{
${ }^{17}$ The use of a second critic was inspired by Double Deep-Q Network [45], or DDQN
} 
in TD3, the actor is updated with a lower frequency than the critics.

$$
\nabla_{\boldsymbol{\theta}_{\pi}} J\left(\boldsymbol{\theta}_{\pi}\right)=\left.\frac{1}{N} \sum \nabla_{a} \hat{\mathrm{Q}}_{1}(s, a)\right|_{a=\pi(s)} \nabla_{\boldsymbol{\theta}_{\pi}} \pi(s)
$$

Eventually, the target networks are updated, as in:

$$
\begin{gathered}
\boldsymbol{\theta}_{\hat{\mathrm{Q}}_{i}^{-}} \longleftarrow \tau \boldsymbol{\theta}_{\hat{\mathrm{Q}}_{i}}+(1-\tau) \boldsymbol{\theta}_{\hat{\mathrm{Q}}_{i}^{-}} \\
\boldsymbol{\theta}_{\pi^{-}} \longleftarrow \tau \boldsymbol{\theta}_{\pi}+(1-\tau) \boldsymbol{\theta}_{\pi^{-}}
\end{gathered}
$$

where $\tau$ is the hyperparameter controlling the speed of the updates.

The complete TD3 algorithm is shown in Algorithm 8. 


\section{Pseudo-code 3.3: Twin Delayed Deep Deterministic Policy Gra-}

\section{dient}

Algorithm 8: Adapted from [44]

Initialise the replay buffer $\mathcal{D}$ with capacity $\mathrm{D}$;

Randomly initialise the critic network $\hat{\mathrm{Q}}_{1}\left(s, a ; \boldsymbol{\theta}_{\mathrm{Q}_{1}}\right)$ and $\hat{\mathrm{Q}}_{2}\left(s, a ; \boldsymbol{\theta}_{\mathrm{Q}_{2}}\right)$ with weights $\boldsymbol{\theta}_{\hat{\mathrm{Q}}_{1}}$ and $\boldsymbol{\theta}_{\mathrm{Q}_{2}}$, and the actor network $\pi\left(s ; \boldsymbol{\theta}_{\pi}\right)$ with weights $\boldsymbol{\theta}_{\pi}$

Initialise the target networks ${\hat{Q_{1}}}^{-}, \hat{\mathrm{Q}}_{2}^{-}$, and $\pi^{-}$with weights $\boldsymbol{\theta}_{\hat{\mathrm{Q}}_{1}^{-}} \longleftarrow \boldsymbol{\theta}_{\hat{\mathrm{Q}}_{1}}, \boldsymbol{\theta}_{\hat{\mathrm{Q}}_{2}^{-}} \longleftarrow \boldsymbol{\theta}_{\hat{\mathrm{Q}}_{2}}$, and $\boldsymbol{\theta}_{\pi^{-}} \longleftarrow \boldsymbol{\theta}_{\pi} ;$

for episode $=0, M-1$ do

initialise a random process $\mathcal{N}$ for action exploration;

Set episode initial state $S_{0}=s_{\text {init }}$;

for $t=0, T-1$ do

Select action $A_{t}=\pi\left(S_{t} ; \boldsymbol{\theta}_{\pi}\right)+\mathcal{N} ;$

Execute action $A_{t}$ and observe reward $R_{t}$ and next state $S_{t+1}$;

Store transition $\left(S_{t}, A_{t}, R_{t}, S_{t+1}\right)$ in $\mathcal{D}$;

Sample random mini-batch of transitions $\left(S_{j}, A_{j}, R_{j}, S_{j+1}\right)$ from $\mathcal{D}$;

$\tilde{A}_{t}=\pi^{-}(s)+\epsilon, \quad \epsilon \sim \operatorname{clip}(\mathcal{N}(0, \tilde{\sigma}),-c, c) ;$

Compute target

$$
y_{j}= \begin{cases}R_{j}, & \text { for terminal } S_{j} \\ R_{j}+\gamma \min _{i=1,2} \hat{\mathrm{Q}}_{i}^{-}\left(s^{\prime}, \tilde{a} ; \boldsymbol{\theta}_{\hat{\mathrm{Q}}_{i}^{-}}\right), & \text {otherwise }\end{cases}
$$

Update the critics networks parameters by:

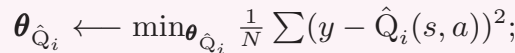

if $t \bmod d$ then

Update the actor network using the deterministic policy gradient:

$\left.\left.\nabla_{\boldsymbol{\theta}_{\pi}} J \approx \frac{1}{N} \sum_{j} \nabla_{a} \hat{\mathrm{Q}}\left(s, a ; \boldsymbol{\theta}_{\mathrm{Q}}\right)\right|_{s=S_{j}, a=\pi\left(S_{j}\right)} \nabla_{\boldsymbol{\theta}_{\pi}} \pi\left(S_{j} ; \boldsymbol{\theta}_{\pi}\right)\right|_{s=S_{j}} ;$

Update the target networks:

$\boldsymbol{\theta}_{\hat{\mathrm{Q}}^{-}} \longleftarrow \tau \boldsymbol{\theta}_{\hat{\mathrm{Q}}}+(1-\tau) \boldsymbol{\theta}_{\hat{\mathrm{Q}}^{-}}$

$\boldsymbol{\theta}_{\hat{\pi}^{-}} \longleftarrow \tau \boldsymbol{\theta}_{\pi}+(1-\tau) \boldsymbol{\theta}_{\hat{\pi}^{-}} ;$

end

end

end 


\subsubsection{Proximal Policy Optimisation}

Proximal Policy Optimisation [46], or PPO, is an on-policy actor-critic Deep Reinforcement Learning algorithm based on policy gradient and trust-region optimisation methods ${ }^{18}$, such as the Trust-Region Policy Optimisation [47], or TRPO. PPO and TRPO jointly ascend the gradient of the policy $\nabla_{\boldsymbol{\theta}} J(\boldsymbol{\theta})$ (see Chapter 2, Section 2.2.2), while bounding its update within the trust-region. They aim at improving the performance of the policy but also constraining each update of the policy to the old one to prevent policy-collapsing ${ }^{19}$. Both algorithms estimate policy and value function using neural networks.

TRPO aims at maximising the policy ${ }^{20}$ performance $J_{\text {old }}(\boldsymbol{\theta})$, while constraining the update of the policy by means of the Kullback-Leibler, or KL, divergence ${ }^{21}$ between the parametrised policy $\pi(a \mid s ; \boldsymbol{\theta})$ and the old policy $\pi_{\text {old }}\left(a \mid s ; \boldsymbol{\theta}_{\text {old }}\right)$, i.e. the policy parametrised by the parameter vector $\boldsymbol{\theta}_{\text {old }}$ before the update. This is shown in Equation (3.16).

$$
\begin{array}{cl}
\max _{\boldsymbol{\theta}} & J_{\text {old }}(\boldsymbol{\theta}) \\
\text { subject to } & \left.D_{\mathrm{KL}}\left[\pi_{\text {old }}\left(A_{t} \mid S_{t} ; \boldsymbol{\theta}_{\text {old }}\right), \pi\left(A_{t} \mid S_{t} ; \boldsymbol{\theta}\right)\right)\right] \leq \epsilon
\end{array}
$$

where $\epsilon$ is the parameters determining the maximum allowed change of the policy and $D_{\mathrm{KL}}$ is the KL-divergence operator, and $\left.D_{\mathrm{KL}}\left[\pi_{\text {old }}\left(A_{t} \mid S_{t} ; \boldsymbol{\theta}_{\text {old }}\right), \pi\left(A_{t} \mid S_{t} ; \boldsymbol{\theta}\right)\right)\right]=$ $\mathbb{E}\left[\log \pi_{\text {old }}\left(A_{t} \mid S_{t} ; \boldsymbol{\theta}_{\text {old }}\right)-\log \pi\left(A_{t} \mid S_{t} ; \boldsymbol{\theta}\right)\right]$. The constrained optimisation problem, shown in Equation (3.16), is usually reformulated in the form of a loss of function $\mathcal{L}_{T P R O}(\boldsymbol{\theta})$ and then used to train the policy neural network. The KL-divergence

\footnotetext{
${ }^{18}$ Trust-region optimisation methods constrain the optimiser update, e.g. gradient descent/ascent, to a fixed neighborhood, i.e. the trust-region, of the current solution for a monotonic improvement.

${ }^{19}$ When updating the policy parameters, large update steps may ruin the performance of the agent. Large updates increase the variance of the reward over training and the overall training time as the agent needs additional training steps to recover a good policy.

${ }^{20} \mathrm{TRPO}$ and PPO utilise stochastic policies in the form of probability distributions $\pi(a \mid s ; \boldsymbol{\theta})$.

${ }^{21}$ The Kullback-Leibler divergence is a non-negative measure of the difference between two probability distributions.
} 
becomes a penalty term in the policy gradient loss, as shown in Equation (3.17).

$$
\begin{aligned}
\max _{\boldsymbol{\theta}} & \mathcal{L}_{T P R O}(\boldsymbol{\theta}) \\
\mathcal{L}_{T P R O}(\boldsymbol{\theta})= & \mathbb{E}\left[J_{\text {old }}(\boldsymbol{\theta})-\beta D_{\mathrm{KL}}\left[\pi_{\text {old }}\left(A_{t} \mid S_{t} ; \boldsymbol{\theta}_{\text {old }}\right), \pi\left(A_{t} \mid S_{t} ; \boldsymbol{\theta}\right)\right)\right] \\
= & \mathbb{E}\left[\frac{\pi\left(A_{t} \mid S_{t} ; \boldsymbol{\theta}\right)}{\pi_{\text {old }}\left(A_{t} \mid S_{t} ; \boldsymbol{\theta}_{\text {old }}\right)} \hat{\mathrm{A}}\left(S_{t}, A_{t} ; \boldsymbol{w}\right)-\beta D_{\mathrm{KL}}\left[\pi_{\text {old }}\left(A_{t} \mid S_{t} ; \boldsymbol{\theta}_{\text {old }}\right), \pi\left(A_{t} \mid S_{t} ; \boldsymbol{\theta}\right)\right)\right]
\end{aligned}
$$

where $\hat{\mathrm{A}}(s, a ; \boldsymbol{w})=\hat{\mathrm{Q}}\left(s, a ; \boldsymbol{w}_{\mathrm{Q}}\right)-\hat{\mathrm{V}}\left(s ; \boldsymbol{w}_{\mathrm{V}}\right)$ is the estimation of the so-called advantage-value function emphasizing the advantage of taking action $a$ is the state $s$, and $\beta$ is a scaling factor.

PPO replaces the KL-divergence, expensive to compute, with a simple clipping of the magnitude of the policy update, as shown in Equation (3.18).

$$
\begin{gathered}
\max _{\boldsymbol{\theta}} \quad \mathcal{L}_{\text {clip }}(\boldsymbol{\theta}) \\
\mathcal{L}_{\text {clip }}(\boldsymbol{\theta})=\mathbb{E}\left[\min \left(\frac{\pi\left(A_{t} \mid S_{t} ; \boldsymbol{\theta}\right)}{\pi_{\text {old }}\left(A_{t} \mid S_{t} ; \boldsymbol{\theta}_{\text {old }}\right)} \hat{\mathrm{A}}\left(S_{t}, A_{t} ; \boldsymbol{w}\right), \pi_{\epsilon}\left(A_{t} \mid S_{t} ; \boldsymbol{\theta}\right) \hat{\mathrm{A}}\left(S_{t}, A_{t} ; \boldsymbol{w}\right)\right)\right] \\
\text { with } \quad \pi_{\epsilon}\left(A_{t} \mid S_{t} ; \boldsymbol{\theta}\right)=\operatorname{clip}\left(\frac{\pi\left(A_{t} \mid S_{t} ; \boldsymbol{\theta}\right)}{\pi_{\text {old }}\left(A_{t} \mid S_{t} ; \boldsymbol{\theta}_{\text {old }}\right)}, 1-\epsilon, 1+\epsilon\right)
\end{gathered}
$$

where $\epsilon$ is the clipping coefficient determining the maximum accepted magnitude of changes in the policy. PPO learns the advantage function by simply estimating the value function $\hat{\mathrm{V}}(s ; \boldsymbol{w})^{22}$ and the infer the truncated advantage value function as:

$$
\begin{aligned}
\hat{\mathrm{A}}\left(S_{t} ; \boldsymbol{w}\right) & =\delta_{t}+\gamma \delta_{t+1}+\cdots+\gamma^{T-t+1} \delta_{T-1} \\
\delta_{t} & =R_{t}+\gamma \hat{\mathrm{V}}\left(S_{t+1} ; \boldsymbol{w}\right)-\hat{\mathrm{V}}\left(S_{t} ; \boldsymbol{w}\right)
\end{aligned}
$$

where $\gamma$ is the discount factor and $T$ is the number of time-steps the policy has $\operatorname{run}^{23}$.

The PPO algorithm is shown in Algorithm 9.

\footnotetext{
${ }^{22}$ The value function $\hat{\mathrm{V}}(s ; \boldsymbol{w})$ can be estimated using the approaches described in previous sections.

${ }^{23}$ We typically choose $T$ much smaller than the whole episode length.
} 


\section{Pseudo-code 3.4: Proximal Policy Optimisation}

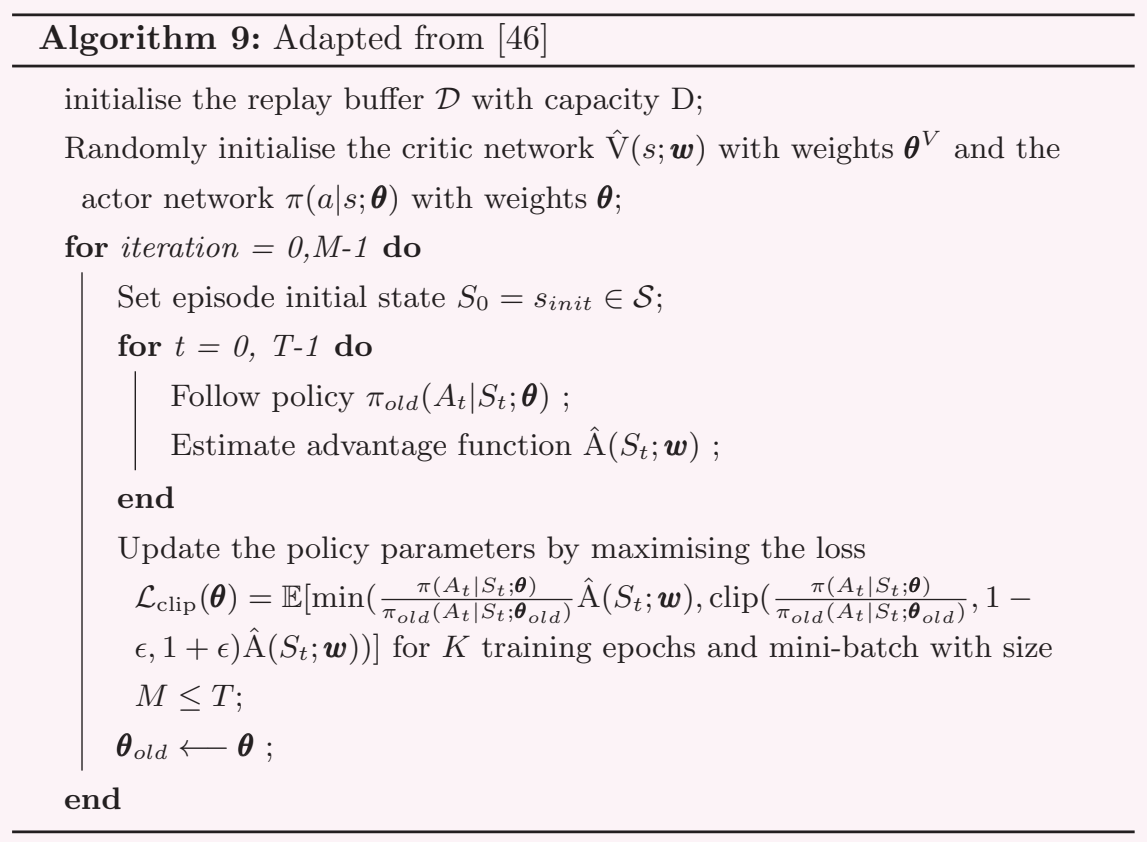

\subsection{Representation Learning for Deep Reinforce- ment Learning}

Deep Reinforcement Learning has been used for solving decision making problems when the state of the environment is not directly observable ${ }^{24}$, and highdimensional observations are received by the agent (e.g. RGB images or LiDAR readings) without the need for any form of hand-crafted feature engineering, i.e. no need of prior knowledge of the environment or the task, and data pre-processing to extract relevant features from the input data (see Figure 3.5). Deep Reinforcement Learning algorithms can learn to directly map high-dimensional observations (e.g. robot's raw sensory readings), obtained through the interaction with the environment, to the actions by solely looking at the rewards received. The direct end-to-end mapping from observation to action has successfully solved a huge

\footnotetext{
${ }^{24}$ We refer to the case of Partially Observable Markov Decision Processes presented in Section 2.1 .3
} 
variety of tasks, e.g. in video-games and robotics [41], [48], [49], [50] and [51], but it usually requires a high amount of data, long training time and high-end computational resources (e.g. multiple CPUs, and GPUs and abundant memory). Moreover, samples are not often cheap and easy to obtain, for example when training on real robotics hardware.

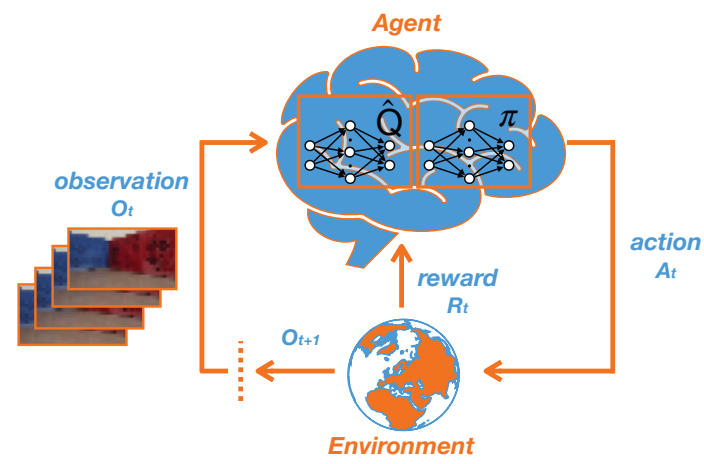

Figure 3.5: End-to-end Deep Reinforcement Learning scheme with deep neural networks.

In many Deep Reinforcement Learning problems, the observation space may have a dimensionality several orders of magnitude higher than the true underlying state of the environment. For example, suppose an agent is controlling a ground mobile robot to navigate to a given target location given RGB images of size $32 \times 32$, the observation space has a dimensionality equal to $32 \times 32 \times 3=3072^{25}$, while the set of minimum information for solving a navigation task corresponds to the pose of the robot, which has dimensionality equal to 3 , i.e. $(x, y, \vartheta)$ respectively position and orientation on the 2D-plane. With this in mind, can we do better than learning end-to-end? Independently of the dimension of the observation, can we enforce specific properties (e.g. dimensionality) of the agent's state? Can we incorporate in this process prior knowledge of the world and the task?

State Representation Learning [6], or SRL, is the name given to the process of learning to encode the task-meaningful information from the high-dimensional observations received by the agent into a lower-dimensional state representation. The Reinforcement Learning agent then uses the learned state representation to

\footnotetext{
${ }^{25}$ An RGB image is composed of three channels: Red, Green and Blue. That is why, to compute its overall size, we multiply by three.
} 
find the optimal policy and value function. This process is shown in Figure 3.6. In contrast with Figure 3.5, here we aim at explicitly separating the learning of the state features, using the neural network $\phi_{e}$, with the learning of the optimal policy and value function. High-dimensional data are usually very rich in information and

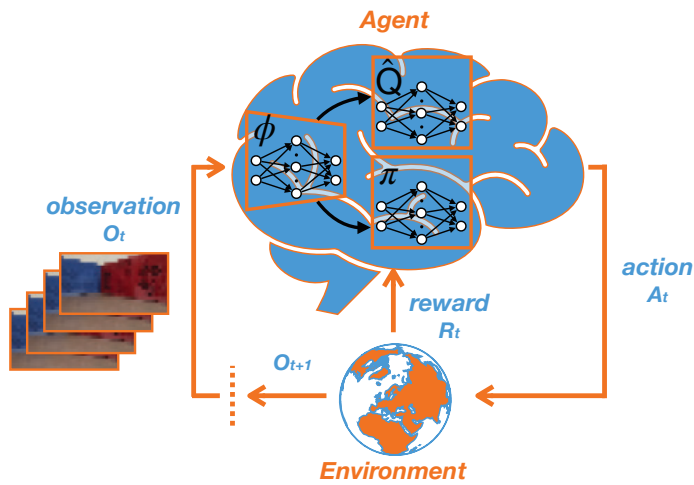

Figure 3.6: End-to-end Deep Reinforcement Learning scheme with deep neural networks.

features. Representing this data into lower-dimensional spaces helps to remove irrelevant features, exploiting similarities and symmetries, and identify hidden structures. The quality of the state representation is crucial for decision-making and affects the performances and generalization capabilities of the Reinforcement Learning algorithms.

In this section, we focus on the case in which the mapping from observations to states is learned using a neural network $\phi_{e}$, but without the use of labelled data ${ }^{26}$. We make only use of the experience tuple $\left(o_{t}, a_{t}, o_{t+1}, r_{t}\right)$ collected through the interaction with the environment.

\subsubsection{Unsupervised Learning of State Representations with Neural Networks}

Accordingly to [6], three main methodologies can be followed to learn low-dimensional state representations for Reinforcement Learning ${ }^{27}$ with neural networks, trained

\footnotetext{
${ }^{26}$ This is often the case in Reinforcement Learning in which labelled data is not present.

${ }^{27}$ For the sake of simplicity the theory presented discusses the case of deterministic environments, but this can be easily extended to the case of stochastic ones.
} 
in unsupervised or self-supervised ${ }^{28}$ fashion. In this section, for the sake of conciseness, we focus on the case of learning deterministic mappings from observations to the agent's states, but the methods proposed here can be adapted to deal with stochastic mappings as well.

Independently of the chosen method ${ }^{29}$, the state representation should efficiently compress the observation space, with minimum information loss, to a state space with Markovian properties [52], i.e. the single state prediction $\hat{s}$ needs to contain enough information for choosing the best action without ambiguity. Additionally, the state representation should also be able to generalize to unseen observations with similar features and to be robust against perturbations of the input data. Eventually, the state representation learning step often increases the interpretability of the learned features and (partially) opens the black-box of the Reinforcement Learning algorithms.

\section{Learning State Representation via Auto-Encoders}

The first class of methods relies on the observation reconstruction using, for example, Auto-Encoders [53], or AEs. An AE is a neural network composed of an encoder $\phi_{e}$, with parameters' vector $\boldsymbol{\theta}_{e}$, and a decoder $\phi_{d}$, with parameters' vector $\boldsymbol{\theta}_{d}$. The encoder maps the input data $x$ to a low-dimensional ${ }^{30}$ latent or abstract code $h=\phi_{e}\left(x ; \boldsymbol{\theta}_{e}\right)$, while the decoder tries to reconstruct the input signal $\hat{x}=\phi_{d}\left(h ; \boldsymbol{\theta}_{d}\right)$ given the code $h$. The AE is trained by minimising the so-called reconstruction $\operatorname{loss}^{31}$, e.g. the MSE loss between the original input and the reconstructed one, as shown in Equation (3.20).

$$
\begin{aligned}
\min _{\boldsymbol{\theta}_{e}, \boldsymbol{\theta}_{d}} & \mathcal{L}_{A E}\left(\boldsymbol{\theta}_{e}, \boldsymbol{\theta}_{d}\right) \\
\mathcal{L}_{A E}\left(\boldsymbol{\theta}_{e}, \boldsymbol{\theta}_{d}\right)= & \frac{1}{b} \sum_{i=1}^{b}\left[\left\|x^{(i)}-\hat{x}^{(i)}\right\|^{2}\right]=\mathbb{E}\left[\left\|x-\phi_{d}\left(\phi_{e}\left(x ; \boldsymbol{\theta}_{e}\right) ; \boldsymbol{\theta}_{d}\right)\right\|^{2}\right]
\end{aligned}
$$

\footnotetext{
${ }^{28}$ In state representation learning for Reinforcement Learning, the training data-set is composed of experience tuples $\left(o_{t}, a_{t}, r_{t}, o_{t+1}\right) \forall t$. For example, we can use the next observation $o_{t+1}$ to create a target prediction to compute the loss of the neural network when fed with the current observation $o_{t}$. This procedure is often used when learning the forward or transition model of the MDPs.

${ }^{29}$ The three approaches described above can be used, and are often used, in combination with each other.

${ }^{30}$ This bottleneck is used to prevent to learn the trivial identity mapping from input to output.

${ }^{31}$ As in previous sections, we assume the existence of a training set of size $l$. The network is trained by ramdomly sampling mini-batches of size $b \ll l$.
} 
where $x$ is the original input data, and $\hat{x}$ is its reconstruction using the AE.

In Deep Reinforcement Learning, we can use the encoder $\phi_{e}$ to map an observation $o$ to a latent state variable $\hat{s}=\phi_{e}\left(o ; \boldsymbol{\theta}_{e}\right)$ of lower dimensionality, and then reconstruct the observation $\hat{o}=\phi_{d}\left(\hat{s} ; \boldsymbol{\theta}_{d}\right)$ from the latent state variable $\hat{s}$ in order to compute the reconstruction loss in Equation (3.20). This is shown in Figure 3.7. The latent state variables are then used to learn policies and value functions. AE

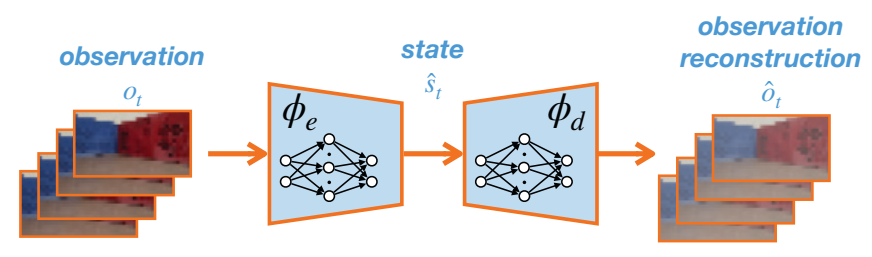

Figure 3.7: Auto-Encoder for Deep Reinforcement Learning.

learning methods for state representation in Reinforcement Learning are proposed in [50], [54], [55], and [56]. Many variations of AEs have been proposed in the literature, such as Variational Autoencoders [57], or VAEs, for learning stochastic mappings, and Denoising Autoencoders [58] for dealing with noise-corrupted input data. For more information, we refer the reader to [34].

\section{Learning State Representation via Learned MDP Models}

When the relevant features to encode into the learned state space are not the most salient features of our data ${ }^{32}$, AEs-only methods tend to fail in capturing good state representations. Alternatively, it is possible to learn the state representation, i.e. learn the optimal parameters for the encoder network $\phi_{e}$, by employing auxiliary loss functions based on the MDP models, e.g. transition, inverse and reward models, as shown in Figure 3.8. These models are trained by making full use of the experience tuple $\left(o_{t}, a_{t}, o_{t+1}, r_{t}\right)$ collected through the interaction with the environment.

The first auxiliary loss, shown in Equation (3.21), for training the encoder $\phi_{e}$ makes use of the latent forward or transition model of the MDP, modelled by a

\footnotetext{
${ }^{32}$ Images may contain many features, such as background textures, that are not relevant for learning the optimal policy, yet important for minimising the reconstruction loss.
} 


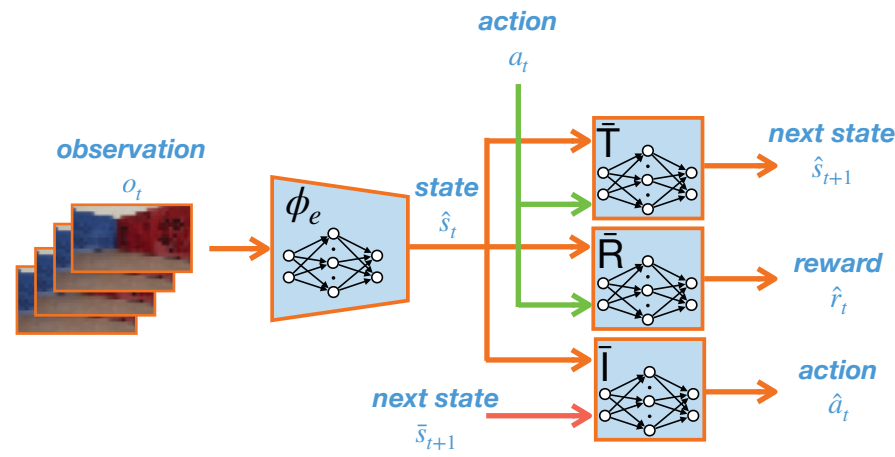

Figure 3.8: State representation learning for Deep Reinforcement Learning using MDP models' losses.

neural network with parameters' vector $\boldsymbol{\theta}_{\overline{\mathrm{T}}}$. A latent transition model predicts the next latent state $\hat{s}_{t+1}$ given the current latent state $\hat{s}_{t}=\phi_{e}\left(o_{t} ; \boldsymbol{\theta}_{e}\right)$ and action $a_{t}$ taken by the agent.

$$
\begin{aligned}
& \min _{\boldsymbol{\theta}_{e}, \boldsymbol{\theta}_{\overline{\mathrm{T}}}} \mathcal{L}_{\mathrm{T}}\left(\boldsymbol{\theta}_{e}, \boldsymbol{\theta}_{\overline{\mathrm{T}}}\right) \\
& \mathcal{L}_{\overline{\mathrm{T}}}\left(\boldsymbol{\theta}_{e}, \boldsymbol{\theta}_{\overline{\mathrm{T}}}\right)=\mathbb{E}\left[\left\|\bar{s}_{t+1}-\hat{s}_{t+1}\right\|^{2}\right]=\mathbb{E}\left[\left\|\phi_{e}\left(o_{t+1} ; \boldsymbol{\theta}_{e}\right)-\overline{\mathrm{T}}\left(\phi_{e}\left(o_{t} ; \boldsymbol{\theta}_{e}\right), a_{t} ; \boldsymbol{\theta}_{\overline{\mathrm{T}}}\right)\right\|^{2}\right]
\end{aligned}
$$

where the target next latent state $\bar{s}_{t+1}=\phi_{e}\left(o_{t+1} ; \boldsymbol{\theta}_{e}\right)$ is generated by encoding the true next observation $o_{t+1}$, while $\hat{s}_{t+1}=\overline{\mathrm{T}}\left(\phi_{e}\left(o_{t} ; \boldsymbol{\theta}_{e}\right), a_{t} ; \boldsymbol{\theta}_{\overline{\mathrm{T}}}\right)$ is the predicted next latent state given the current observation $o_{t}$ and the action taken $a_{t}$.

Similarly, we can learn a latent inverse model, modelled by a neural network with parameters' vector $\boldsymbol{\theta}_{\overline{\mathrm{I}}}$, to predict the action $\hat{a}_{t}$ connecting the current latent state $\hat{s}_{t}$ and the next latent state $\bar{s}_{t+1}$. An example of inverse model loss for training the encoder $\phi_{e}$ is shown in Equation (3.22).

$$
\begin{aligned}
& \min _{\boldsymbol{\theta}_{e}, \boldsymbol{\theta}_{\overline{\mathrm{I}}}} \mathcal{L}_{\overline{\mathrm{I}}}\left(\boldsymbol{\theta}_{e}, \boldsymbol{\theta}_{\overline{\mathrm{I}}}\right) \\
& \mathcal{L}_{\overline{\mathrm{I}}}\left(\boldsymbol{\theta}_{e}, \boldsymbol{\theta}_{\overline{\mathrm{I}}}\right)=\mathbb{E}\left[\left\|a_{t}-\hat{a}_{t}\right\|^{2}\right]=\mathbb{E}\left[\left\|a_{t}-\overline{\mathrm{I}}\left(\phi_{e}\left(o_{t} ; \boldsymbol{\theta}_{e}\right), \phi_{e}\left(o_{t+1} ; \boldsymbol{\theta}_{e}\right) ; \boldsymbol{\theta}_{\overline{\mathrm{I}}}\right)\right\|^{2}\right]
\end{aligned}
$$

Eventually, we can make use of a reward model to predict the next reward given the current latent state $\hat{s}_{t}$ and the action ${ }^{33}$ taken $a_{t}$. An example of reward

\footnotetext{
${ }^{33}$ The reward model may be only a function of the latent state $\hat{s}_{t}$.
} 
model loss is shown in Equation (3.23).

$$
\begin{aligned}
& \min _{\boldsymbol{\theta}_{e}, \boldsymbol{\theta}_{\overline{\mathrm{R}}}} \mathcal{L}_{\overline{\mathrm{R}}}\left(\boldsymbol{\theta}_{e}, \boldsymbol{\theta}_{\overline{\mathrm{R}}}\right) \\
& \mathcal{L}_{\overline{\mathrm{R}}}\left(\boldsymbol{\theta}_{e}, \boldsymbol{\theta}_{\overline{\mathrm{R}}}\right)=\mathbb{E}\left[\left\|r_{t}-\hat{r}_{t}\right\|^{2}\right]=\mathbb{E}\left[\left\|r_{t}-\overline{\mathrm{R}}\left(\phi_{e}\left(o_{t} ; \boldsymbol{\theta}_{e}\right), a_{t} ; \boldsymbol{\theta}_{\overline{\mathrm{R}}}\right)\right\|^{2}\right]
\end{aligned}
$$

Forward, inverse, and reward models are often used together for learning state representations coherent with the MDP underlying model as shown in Equation (3.24). Similar loss functions for learning state representations for Reinforcement Learning are used, for example, in [59], [60], [61], and [62].

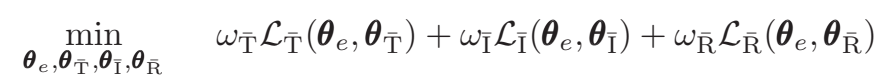

where $\omega_{\overline{\mathrm{T}}}, \omega_{\overline{\mathrm{I}}}$ and $\omega_{\overline{\mathrm{R}}}$ are three scalar factors for scaling the contribution of each individual loss.

\section{Learning State Representation via Robotics Priors}

When the underlying MDP model is fully or partially governed by the laws of physics, such as in robotics, we may want to exploit such knowledge in the state representation learning step. For example, despite being high-dimensional (e.g. robot's sensory readings), the robot's observations are generated from true environment states (e.g. robot's true pose) that evolve according to specific physical laws. We can encode such prior physical knowledge in the form of loss functions and use them for training the neural network encoder $\phi_{e}$ in charge of the observation-state mapping.

These loss functions, often called priors or robotics priors, allow the most straightforward network architecture, as only the encoder $\phi_{e}$ is needed, as shown in Figure 3.8. An example of prior loss is the so-called temporal coherence loss introduced in [63] and shown in Equation (3.25).

$$
\begin{gathered}
\min _{\boldsymbol{\theta}_{e}} \mathcal{L}_{\text {temp }}\left(\boldsymbol{\theta}_{e}\right) \\
\mathcal{L}_{\text {temp }}\left(\boldsymbol{\theta}_{e}\right)=\mathbb{E}\left[\left\|\Delta \hat{s}_{t}\right\|^{2}\right]=\mathbb{E}\left[\left\|\hat{s}_{t+1}-\hat{s}_{t}\right\|^{2}\right]=\mathbb{E}\left[\left\|\phi_{e}\left(o_{t+1} ; \boldsymbol{\theta}_{e}\right)-\phi_{e}\left(o_{t} ; \boldsymbol{\theta}_{e}\right)\right\|^{2}\right]
\end{gathered}
$$

The temporal coherence loss enforces smooth and small changes in the learned state space between pairs of consecutive latent states. The prior knowledge introduced 


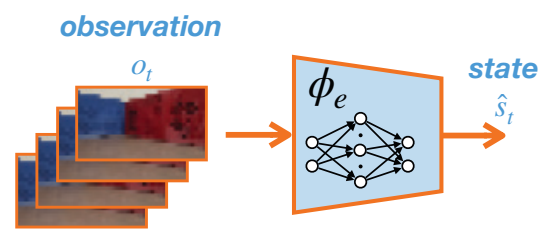

Figure 3.9: State representation learning for Deep Reinforcement Learning using robotics priors.

by this loss function is the assumption that the true state space is smooth and continuous. This property of the state space is true for any environment obeying, for example, to physical laws such as in robotics.

The temporal coherence prior alone is not sufficient for learning an informative state representation as the global minimum of such loss function is reached by mapping each latent state to the zero vector. Therefore, to prevent the learning of the trivial mapping, the causality loss has been introduced in [63], and it is shown Equation (3.26). This prior utilised pairs of states, collected at different time instants $t_{1}$ and $t_{2}$, with same actions $a_{t_{1}}, a_{t_{2}}$ taken from such states, but different rewards $r_{t_{1}}, r_{t_{2}}$ received, and it is minimised when the two states predictions are pushed apart in the latent space.

$$
\begin{aligned}
\min _{\boldsymbol{\theta}_{e}} & \mathcal{L}_{\text {caus }}\left(\boldsymbol{\theta}_{e}\right) \\
\mathcal{L}_{\text {caus }}\left(\boldsymbol{\theta}_{e}\right)= & \mathbb{E}\left[e^{-\left\|\hat{s}_{t_{1}}-\hat{s}_{t_{2}}\right\|^{2}}\right]=\mathbb{E}\left[e^{-\left\|\phi_{e}\left(o_{t_{1}} ; \boldsymbol{\theta}_{e}\right)-\phi_{e}\left(o_{t_{2}} ; \boldsymbol{\theta}_{e}\right)\right\|^{2}} \mid a_{t_{1}}=a_{t_{2}}, r_{t_{1}} \neq r_{t_{2}}\right]
\end{aligned}
$$

When the same actions $a_{t_{1}}, a_{t_{2}}$ are applied in different states, we want our state variations $\Delta \hat{s}_{t_{1}}=\hat{s}_{t_{1}+1}-\hat{s}_{t_{1}}$ and $\Delta \hat{s}_{t_{2}}=\hat{s}_{t_{2}+1}-\hat{s}_{t_{2}}$ to be similar in magnitude. This prior is encoded into the proportionality loss, introduced in [63], and shown in Equation (3.27).

$$
\begin{aligned}
\min _{\boldsymbol{\theta}_{e}} & \mathcal{L}_{\text {prop }}\left(\boldsymbol{\theta}_{e}\right) \\
\mathcal{L}_{\text {prop }}\left(\boldsymbol{\theta}_{e}\right)= & \mathbb{E}\left[\left(\left\|\Delta \hat{s}_{t_{1}}\right\|-\| \Delta \hat{s}_{t_{2}} \mid\right)^{2} \mid a_{t_{1}}=a_{t_{2}}\right]
\end{aligned}
$$

Eventually, to obtain an informative and coherent state representation, the encoder $\phi_{e}$ is then usually trained by minimising multiple prior losses, as shown 
in Equation (3.28).

$$
\min _{\boldsymbol{\theta}_{e}} \omega_{\text {temp }} \mathcal{L}_{\text {temp }}\left(\boldsymbol{\theta}_{e}\right)+\omega_{\text {caus }} \mathcal{L}_{\text {caus }}\left(\boldsymbol{\theta}_{e}\right)+\omega_{\text {prop }} \mathcal{L}_{\text {prop }}\left(\boldsymbol{\theta}_{e}\right)
$$

where $\omega_{\text {temp }}, \omega_{\text {caus }}$ and $\omega_{\text {prop }}$ are scaling factors for scaling the contribution of each prior loss.

Many robotics priors have been proposed in literature and we refer the reader to [63], [64], [65], [66] and [12] for more details.

\subsection{Deep Reinforcement Learning in Robotics}

In the previous sections, we have discussed the Reinforcement Learning framework as a general way for solving sequential decision-making processes. Here, we want to talk about Reinforcement Learning in the context of robotics and, in particular, its challenges, or curses, and how to deal with them.

\subsubsection{Challenges of Robotics Reinforcement Learning Curse of Dimensionality}

The first, and possibly most well-known, curse of Reinforcement Learning is the so-called curse of dimensionality [21], i.e. the exponential explosion of states and actions occurring when discretising high-dimensional continuous spaces. For example, assuming a $n$-dimensional state space discretised into ten tiers or levels ${ }^{34}$, the resulting number of possible states is $10^{n}$. This problem is even more acute in robotics, where the state and action spaces are intrinsically continuous. According to [10], three major categories of approaches are used in robotics to deal with continuous state and action spaces individually or jointly:

- discretisation

- function approximation

- macro-actions

The most obvious solution is discretising state and action spaces and use one of the tabular Reinforcement Learning approaches presented in Section 2.2.

\footnotetext{
${ }^{34}$ We can divide each dimension of the continuous state space into ten intervals.
} 


\section{Example 3.1: Discretisation}

Any continuous mobile robot navigation task can be represented as a gridworld problem, where the robot is constrained to move along the imposed grid and the agent's actions are none others than move-up, move-down, move-left and move-right. A low-level controller can be then used to enforce the motion inside the grid cells. This is shown in Figure 3.10.
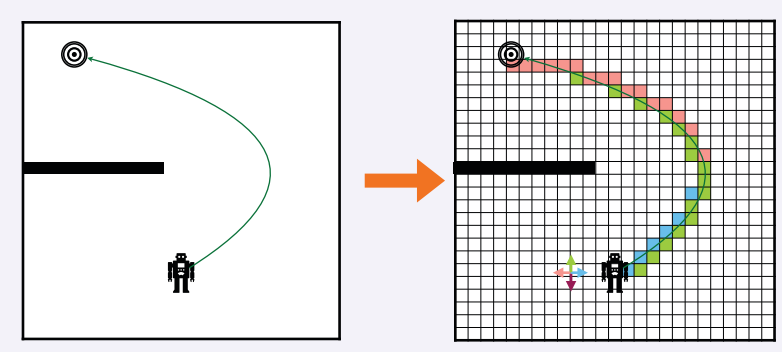

Figure 3.10: Discretisation process.

In this case, the discretisation of the original 2-dimensional state spaces into twenty-five tiers per dimension generates $25^{2}=625$ possible states!

The second option is to directly deal with the continuous nature of the state and action spaces using function approximators and, for example, one of the Deep Reinforcement Learning algorithms discussed throughout Chapter 3. Moreover, when dealing with high-dimensional inputs, such as vision sensory readings, e.g. RGB images, methods employing function approximators need to be used to solve the task. These methods can be further combined with the explicit learning of low-dimensional representations, as presented in Section 3.3.

Eventually, it is possible to decompose the task in simpler and with shorter horizon sub-tasks, that can be more easily solved, using macro-actions or options [67]. 


\section{Example 3.2: Hierarchical Decomposition}

An example of the use of macro-action is shown in Figure 3.11, where the task of reaching the target can be hierarchically decomposed into selecting the room to move in (high-level task) and then move to the chosen room (low-level task). The decomposition allows better generalization property since the low-level policy to move through a room, once learned for a specific room, can be directly reused for any room.

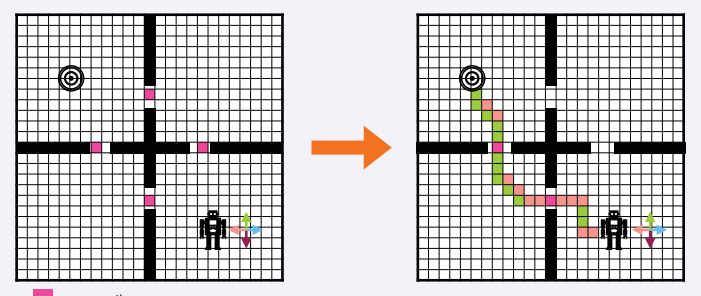

Figure 3.11: Task decomposition via macro-actions.

In the last decade, function approximators have often been employed in robotics Reinforcement Learning to deal with high-dimensional observations and continuous action spaces. However, the discretisation of the action space is often a viable option. Additionally, due to the increasingly complex robotics applications, the hierarchical decomposition of the tasks plays a significant role in efficiently solving these problems with Reinforcement Learning.

\section{Curse of Real-World Samples}

The more the problem complexity grows, the more samples are needed for the Reinforcement Learning algorithms to learn the optimal policy and value function. Robotics problems usually have high complexity, therefore require many training samples. However, robots are physical entities interacting with the real world and, only through interaction with the world, samples can be collected. Unfortunately, robots have costly and fragile hardware that is not normally meant for trial and error. 
For example, consider the case in which Reinforcement Learning is used to learn a navigation policy for a mobile robot. The agent's actions are velocity or torque commands for the actuators of the robot. During the initial phases of the learning, the agent, for learning to avoid collisions with obstacles, has to experience the negative reward obtain by colliding with obstacles. These repeated collisions may damage the robot before the agent can adequately avoid obstacles. This problem is usually referred to as the curse of real-world samples. When learning is directly done in the real world, the Reinforcement Learning algorithms must have high sample efficiency, i.e. the algorithms have to quickly infer the optimal behaviour from a limited set of samples.

Alternatively, it is possible to rely on virtual simulators and physical engines to gather samples without the need for real-world interaction, learn the optimal behaviours using the simulated experience data and then transfer the optimal policy to the real robots, i.e. transfer learning. This is shown in Figure 3.12.

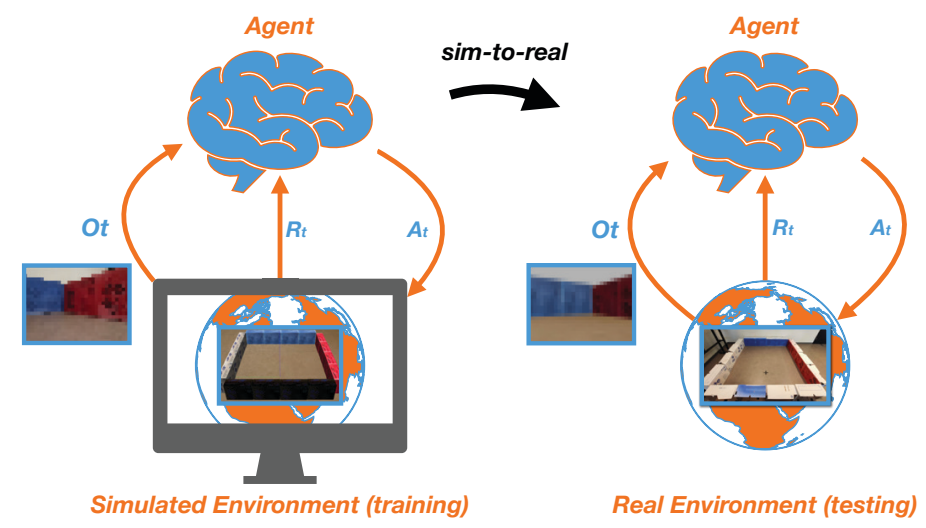

Figure 3.12: Use simulators to learn the optimal behaviour and then test the policy on the real robots without further retraining.

Simulators allow performing experiments that are multiple factors faster than the real-world counterpart. However, simulators use models that are only good approximations of the laws governing the world (e.g. physical laws) or require many data samples to be sufficiently accurate (e.g. data-driven modelling). Suppose the agent's policy is trained using data coming from an inaccurate forward model, the behaviour cannot be transferred to the real case without any re-training or adaptation [68]. Especially for intrinsically unstable dynamical tasks (e.g. inverted 
pendulum stabilisation), transfer learning is often a brittle approach [10] as minor discrepancies in the modelling can produce significant differences in the response of the system. Nonetheless, with increasingly complex problems, simulators play a crucial role in robotics Reinforcement Learning. In the case in which simulators are employed, the Reinforcement Learning algorithms have to be able to learn robust behaviours that can compensate for imperfect modelling (e.g. of robots and sensing) and uncertainties, and that can therefore bridge the simulator-to-realitygap.

\section{Curse of the Goal Specification}

The goal of a Reinforcement Learning agent implicitly defines the reward function. Suppose the reward function considers only the success or the failure, and the agent receives the reward only at the end of the training episode. We talk in these cases of sparse reward functions. Reinforcement Learning agents tend to require lengthy training times when they rarely experience a variation of the reward received.

Alternatively, more informative reward functions can be used to speed up the learning (e.g. reward functions considering side objectives for the agent). We speak, in these cases, about dense reward functions and Reward Shaping [69], [70].

\section{Example 3.3: Reward Shaping}

For example, if we consider the navigation task in the grid world shown in Figure 3.13 if the distance to the target is included in the reward function, the agent can learn the optimal policy faster than in the case of a spare reward penalising collisions with obstacles and rewarding reaching the target location. This happens because the agent can constantly notice variations of the reward function for each action taken. 


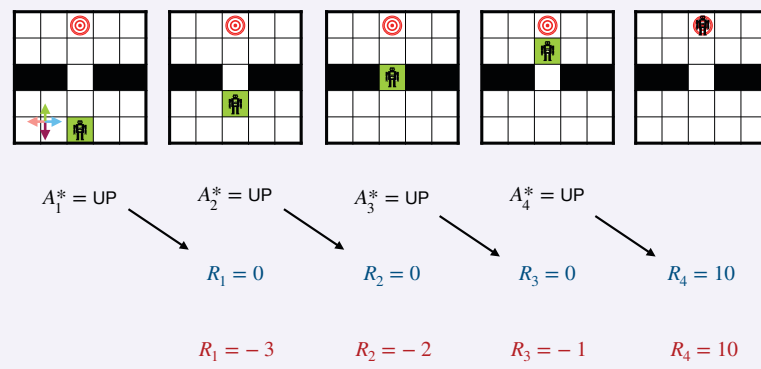

Figure 3.13: Reward received by following the optimal policy in the case of a sparse reward function (blue) and a dense reward function based on the negative Manhattan distance from the agent's current position and the target location (red).

However, for complicated problems, the reward function grows in complexity too, and trade-offs have to be found between the reward-complexity and the task-complexity. Complex and high-dimensional reward functions with multiple weighted objectives (e.g. avoiding collisions, minimising control inputs, reaching a target, or smoothing the trajectories of the robot) usually present many local-minima, i.e. flat regions of the reward function landscape corresponding to sub-optimal behaviours, that Reinforcement Learning algorithms often find and get-stuck into them.

In the case of expert demonstrations ${ }^{35}$ being available, it is possible to infer the reward function from the observed optimal behaviour. This approach is know as inverse Reinforcement Learning [71]. After learning the reward function, this can be used to train the Reinforcement Learning agent to reproduce the expert demonstration. Despite the success of inverse Reinforcement Learning in many different scenarios (e.g. manipulation, navigation and legged-robot locomotion), this approach is limited by the need for suitable expert demonstrations ${ }^{36}$. Moreover, many reward functions can be inferred from the same set of expert data, making the optimal policy prone to learn sub-optimal behaviours.

\footnotetext{
${ }^{35}$ Data collected by a human operator manually controlling the robot is an example of expert demonstration.

${ }^{36}$ For example, for inferring a suitable reward function for a legged-robot, it is usually not enough to provide a sequence of observations of a dog walking. Because of the difference in the geometry of the bodies, sub-optimal behaviour may be learned.
} 


\section{Curse of the Prior Knowledge}

Despite not being explicitly addressed as a curse, the use of prior knowledge in Reinforcement Learning is usually a cause of debate. This dilemma is even more emphasised in the context of robotics Reinforcement Learning, as robotics is traditionally a knowledge-based discipline.

Many forms of prior knowledge can be used in Reinforcement Learning, such as using expert demonstrations and data to initialise policies and value functions and directing the policy updates, smartly decomposing the task and shaping reward functions.

In the case in which expert data are available, it is possible to employ the so-called imitation learning [72] where the Reinforcement Learning policies are trained with supervised learning to behave according to the expert demonstrations. If the agent ends up in states where no expert data are available, it is either possible to provide additional expert data for the new situations or allow the agent to autonomously learn by trial and error, i.e. apprenticeship learning [73].

Alternatively, it is possible to inject expert knowledge by smartly initialising policies and value functions such that sub-optimal behaviours can be immediately achieved. Expert demonstrations and hand-crafted policies not only speed up the learning but also allow to avoid exploring the whole state space by biasing the policies around the expected ${ }^{37}$ good situations. However, because the policies are usually improved in the neighbourhood of the expert policy, these methods tend to find local optimal solutions and rarely global optimal ones. Moreover, for highly complicated tasks, it is difficult, expensive or even impossible to provide complete prior knowledge to the Reinforcement Learning algorithm beforehand.

For complicated problems, prior knowledge can be used to find the task's hierarchical decompositions into progressively simpler sub-tasks. This field of studies is commonly known as hierarchical Reinforcement Learning [74]. Hierarchical Reinforcement Learning methods are founded on the theory of the SMDP, discussed in Section 2.1.4. For example, consider the case of a mobile robot navigating in indoor environments equipped with a robot arm to open doors to move to different rooms. It is possible to simplify the problem by intuitively define two different sub-tasks and sub-policies, namely navigate and open door, and an overall policy

\footnotetext{
${ }^{37}$ Given the prior knowledge.
} 
is simply choosing which of the sub-policies to actuate in the different situations ${ }^{38}$.

Eventually, the most straightforward way to incorporate prior knowledge is to shape the reward functions that the Reinforcement Learning agents maximise. Reward functions can be shaped based on environmental features (e.g. distance to the target and obstacles in a navigation task), but also gradually made more complicated the more the agent has learned (e.g. in a navigation task, we can first only penalise collisions, then we can reward getting close to the target, and eventually we can penalise the high accelerations or velocities) or shaped to promote exploration of the unknown. The latter form of reward-shaping strategy is addressed in literature as curiosity-driven [75], [76].

While prior knowledge may be beneficial for solving specific problems, it may be detrimental for others. When aiming at learning behaviours on real robots, it is, in most cases, necessary to heavily rely on prior knowledge to make the learning safe and sample efficient, even at the price of learning sub-optimal behaviours.

When relying on virtual simulators for training the Reinforcement Learning agents, the boundary between using or not using prior knowledge and to what extent is more blurred. In this context, we may completely avoid the use of any prior knowledge and provide more data to the agents. However, this procedure extends the training time and demands high computational resources that may not always be available. On the other side, simulators allow extensive exploration of the state and action spaces, thus the possibility to find global optimal policies that the heavy use of prior knowledge may limit.

Therefore, we believe that loose forms of prior knowledge should be employed when using simulators. With loose prior knowledge, we refer to all the information that can aid the learning of good features and policies without constraining the solution space with labelled data or models. For example, we consider the process of enforcing properties of the learned state representation, such as dimensionality or smoothness, and reward shaping forms of loose prior knowledge. These approaches allow learning robust representations of the world that better incorporate the actual meaningful information for solving the Reinforcement Learning problems and facilitate the transfer of the learning from the simulators to real-world $\operatorname{robots}^{39}$.

\footnotetext{
${ }^{38}$ The sub-policies can be further decomposed in increasingly simpler and smaller problems.

${ }^{39}$ Dear reader, we hope that you have enjoyed the reading so far and that you have learned something new. In the following chapters of this thesis, we will show you the use of loose prior knowledge in the context of robotics Deep Reinforcement Learning through our scientific publications of the past few years.
} 



\section{State and Action Representation Learning}

\section{II}

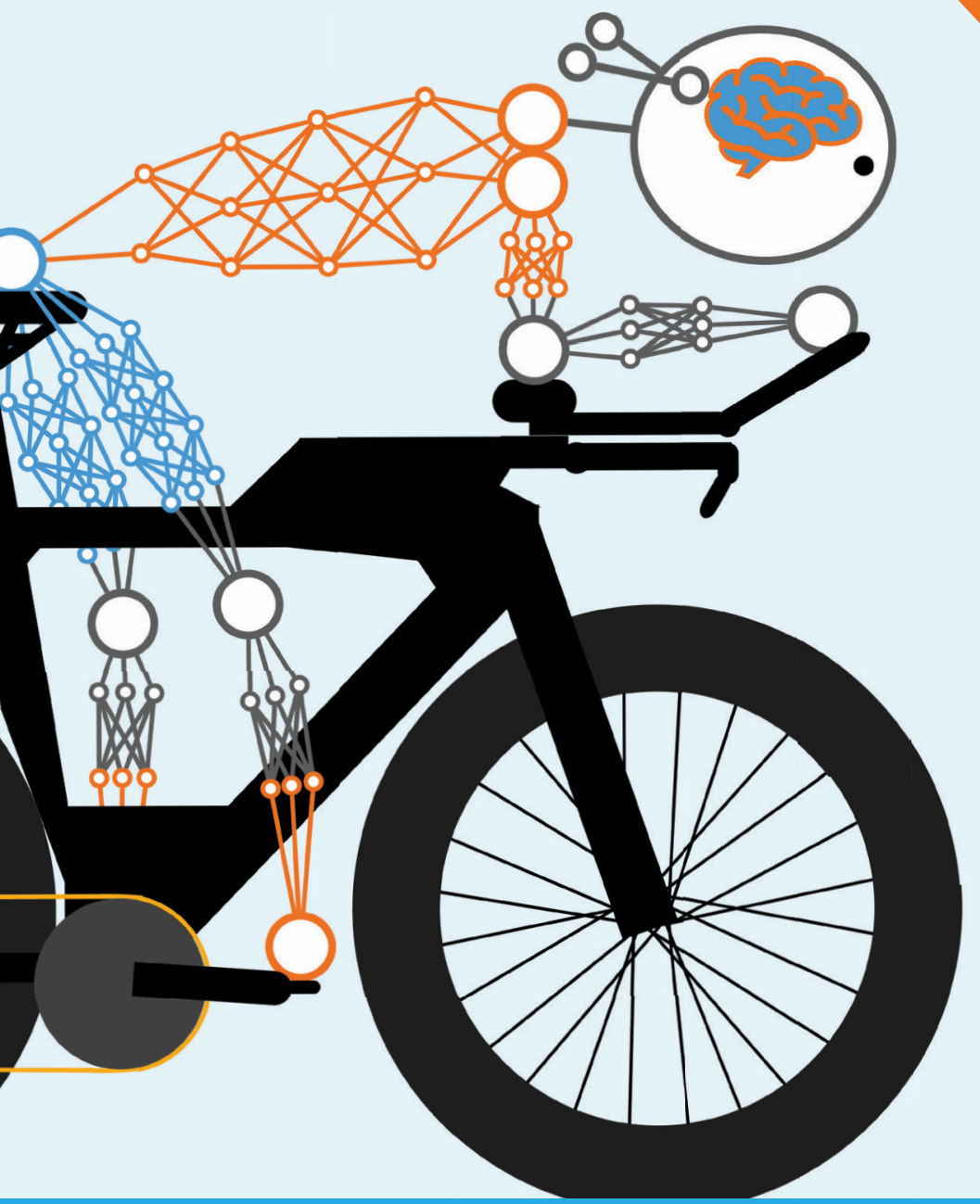





\section{Chapter 4}

\section{Low-Dimensional State Representation Learning with Reward-shaped Priors}

In this chapter, we study the problem of learning an informative state representation for learning robust and transferable navigation policies for steering a mobile robot to different target locations by only relying on its high-dimensional on board sensors. We propose a self-supervised learning method for learning a mapping from the observation space to a lower-dimensional state space by using loss functions shaped to incorporate prior knowledge of the environment and the task. The optimal policy is quickly and efficiently learned using the samples from this learned latent state space. We test the method on several mobile robot navigation tasks in a simulation environment and also on a real robot. A video of our experiments can be found at: https://youtu.be/dgWxmfSv95U.

This chapter includes an adaptation of:

Nicolò Botteghi, Ruben Obbink, Daan Geijs, Mannes Poel, Beril Sirmacek, Christoph Brune, Abeje Mersha, and Stefano Stramigioli. Low-Dimensional State Representation Learning with Reward-shaped Priors. International Conference on Pattern Recognition, 2021.

The Section Background and the Section Related Work were removed as treated in Chapter 2 and 3 , and the notation adapted to be consistent with the notation introduced in this thesis. 


\subsection{Introduction}

Artificial intelligence, or AI, is the key ingredient to bring robots into everyday life. Robots will be asked to accomplish many different and complex tasks, such as navigation and exploration of unknown environments, objects manipulation and human interaction. These challenges require the ability to extract meaningful information or features from the data perceived by the sensors. Because of the high complexity of the tasks, usually, multiple sensor modalities are employed. The so-called observation space, i.e. the space containing what the agent can observe of the environment through, for example, the robot's on board sensors, has a dimensionality much higher than the so-called state space, i.e. the space containing the meaningful information for solving the task.

Traditionally, this leads to complicated manual preprocessing of the data, feature engineering, and coding of the task solution. Even though very successful, feature engineering suffers from a lack of generalisability and reusability in different contexts. For each new task, it is usually necessary a new preprocessing stage and often the coding of a new solution.

State Representation Learning, or SRL, is the name given to the process of learning and encoding the task-meaningful information from the observation space to the so-called state space, i.e. the space containing only the task-relevant information.The mapping from observations to states can be learned using supervised learning methods using labelled data, i.e. the true value of the states. However, these are difficult and expensive to obtain.

In this chapter, we specifically study the problem of learning a state representation using unsupervised or self-supervised learning approaches. However, to aid the learning of a meaningful representation, we use the concept of priors introduced by [77] and further developed by [63]. Using the priors, we model the prior knowledge about the world that can be used to inject information into the state representation learning process. For example, it is possible to phrase these priors as loss functions for training neural networks. Here, we aim at incorporating the reward function properties into the state representation learning problem through the priors. We extend the concept of the priors to multiple sensor modalities ${ }^{1}$, to multi-target navigation tasks and transfer learning from a simulation environment to a real robot. The general framework proposed is shown in Figure 4.1.

\footnotetext{
${ }^{1}$ The use of multiple sensor modalities is a very common scenario in robotics.
} 


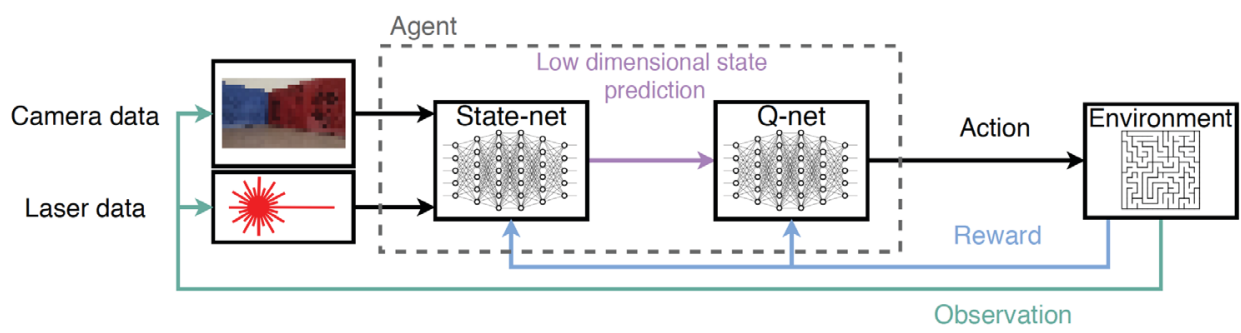

Figure 4.1: End-to-end framework combining State Representation Learning and Reinforcement Learning.

\subsection{Methodology}

\subsubsection{Proposed Approach}

When the state of the environment is not directly observable, but only highdimensional observations are available to the agent, learning informative and meaningful state representations is essential for effective and efficient decision making. However, the mapping from the observation space, usually high-dimensional, to the state space $^{2}$, usually lower-dimensional, is not straightforward. We aim at learning a valid state representation in an unsupervised fashion, using one of the approaches described in Chapter 3, Section 3.3. However, we employ generic domain knowledge to shape the state representation: the robotics priors [63].

With reference to Figure 4.1, the multi-modal observations are fed to the Statenet, in Figure 4.2, i.e. the neural network $\phi_{e}$, parametrised by the parameters' vector $\boldsymbol{\theta}_{e}$, in charge of encoding the important information from the data and compressing them into a lower-dimensional state vector. The State-net design was inspired by the architecture proposed in [78], where the features for each sensor modality are separately processed to make independent state prediction and eventually concatenated to obtain the final state prediction.

The state vector is then passed as input of the Q-net, in Figure 4.3, in order to estimate the state-action value function that is then used to choose the optimal action. DQN was chosen for its simplicity and popularity (see Section 3.2.1). However, the method is not dependent on this choice, and any other Reinforcement Learning algorithm can be used, both with discrete and continuous action spaces.

\footnotetext{
${ }^{2}$ Here, we recall that with state space we intend the space of important environmental information necessary for learning the optimal policy using Reinforcement Learning.
} 


\subsubsection{Reward-shaped Priors}

Our approach builds upon the robotics priors introduced in [63] and aims at addressing the following research questions:

1. How can the reward function, through the priors, be used for shaping the learning of the state representation?

2. How can the concept of priors be extended to multiple sensor modalities, different environments, and multi-targets navigation problems?

3. To what extent can the representation, learned using the priors, in the simulation environment be transferred effectively to the real robot without further re-training?

The priors used in this work to train the State-net are listed below, where $\hat{s}_{t}=\phi_{e}\left(o_{t} ; \boldsymbol{\theta}_{e}\right)$ corresponds to the state prediction given the observation $o_{t}, r_{t}$ to the reward received by taking action $a_{t}$ in the true environment state $s_{t}{ }^{3}$, $\Delta \hat{s}_{t}=\hat{s}_{t+1}-\hat{s}_{t}$ and $\Delta r_{t}=r_{t+1}-r_{t}$.

Simplicity prior: The task-relevant information lies in a space with dimensionality much smaller than the sensory observations.

Temporal Coherence prior: State changes are slow and dependent only on the most recent past. This property can be interpreted as an enforcement of the Markov's assumption.

$$
\mathcal{L}_{1}\left(\boldsymbol{\theta}_{e}\right)=\mathbb{E}\left[\left\|\Delta \hat{s}_{t}^{2}\right\|\right]
$$

Reward Proportionality prior (new): Similar reward changes should induce similar state changes, and these reward changes are the results of actions. However, actions can be continuous or with different levels of abstraction (e.g. in the case of Hierarchical RL), and the notion of similarity is difficult to define for those cases. This new prior aims at clustering together state predictions with similar reward variations independently of the action taken.

$$
\mathcal{L}_{2}\left(\boldsymbol{\theta}_{e}\right)=\mathbb{E}\left[\left(\left\|\Delta \hat{s}_{t_{2}}\right\|-\| \Delta \hat{s}_{t_{1}}||\right)^{2}|| \Delta r_{t_{2}} \sim \Delta r_{t_{1}} \mid\right]
$$

Causality prior (new): Dissimilar rewards are a symptom of state dissimilarity. With analogous reasoning as before, the constraint to similar actions in the

\footnotetext{
${ }^{3}$ Here, the state of the environment is not available, therefore $s_{t}$ is unknown.
} 
Causality prior of [63] is removed.

$$
\mathcal{L}_{3}\left(\boldsymbol{\theta}_{e}\right)=\mathbb{E}\left[e^{-\left\|\hat{s}_{t_{2}}-\hat{s}_{t_{1}}\right\|^{2}} \mid r_{t_{2}} \neq r_{t_{1}}\right]
$$

Reward Repeatability prior (new): Reinforces the similarity of states when presenting the same reward variation, not only in magnitude but also in direction.

$$
\mathcal{L}_{4}\left(\boldsymbol{\theta}_{e}\right)=\mathbb{E}\left[e^{-\left\|\hat{s}_{t_{2}}-\hat{s}_{t_{1}}\right\|^{2}}\left(\left\|\Delta \hat{s}_{t_{2}}-\Delta \hat{s}_{t_{1}}\right\|\right)^{2}|| \Delta r_{t_{2}} \sim \Delta r_{t_{1}} \mid\right]
$$

The overall loss function, in Equation (4.5), that is minimised and used for training the State-Net is equal to the weighted sum of the different priors with the addition of the L2-regularisation term.

$$
\min _{\boldsymbol{\theta}_{e}} \mathcal{L}\left(\boldsymbol{\theta}_{e}\right)=\omega_{1} \mathcal{L}_{1}\left(\boldsymbol{\theta}_{e}\right)+\omega_{2} \mathcal{L}_{2}\left(\boldsymbol{\theta}_{e}\right)+\omega_{3} \mathcal{L}_{3}\left(\boldsymbol{\theta}_{e}\right)+\omega_{4} \mathcal{L}_{4}\left(\boldsymbol{\theta}_{e}\right)+\omega_{5} \mathcal{L}_{r e g}\left(\boldsymbol{\theta}_{e}\right)
$$

where the weights of the single loss functions (in Equation (4.5)) were chosen equal to $\omega_{1}=3, \omega_{2}=15, \omega_{3}=15, \omega_{4}=15$ and $\omega_{5}=3$ to balance the contribution of the each individual term. This combination gave good empirical results, but no optimisation procedure was run to find the best set of weights, and only gridsearch was employed. This means that the approach is not heavily affected by the choice of the weights $\omega$.

In Reinforcement Learning, the reward function is defined and, often shaped, using on task-specific knowledge to allow the agent to learn optimal behaviours. However, in the context of state representation learning, we argue that a task cannot be efficiently learned if an informative representation has not been learned yet. We believe that the best representation is the one that incorporates meaningful information for solving the task. Therefore, it should not be learned independently from the chosen reward function. The new priors, in Equation (4.2), (4.3), and (4.4), were developed to achieve this goal: shaping the state representation using not only the environment observations but also the rewards. In particular, the reward variation from two states is used to impose the Markov's assumption during the observation-compression step. Ideally, we would like to obtain a regularised state space that is Markovian. Given a Markovian state representation, any standard Reinforcement Learning algorithm can optimally solve the problem without any memory structure by looking at a single state prediction. 


\subsubsection{Neural Network Architectures and Training Regime}

The State-Net, Figure 4.2, is an encoder network, i.e. neural network with output dimensionality smaller than the input dimensionality. The samples from the two

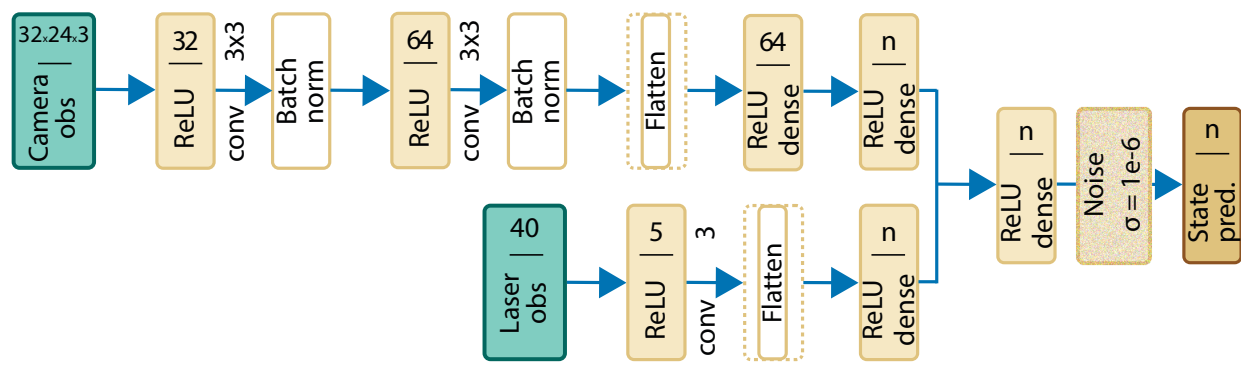

Figure 4.2: Architecture of the State-net.

sensor modalities are processed through two separate network branches, and they are both used to make independent state predictions of dimension $n$. The two predictions are concatenated and then fed through a final fully connected (dense) layer that combines them to produce the final state prediction, again of dimension $n$. The considerations on choice of the state dimensionality are shown in Section 4.4. The state predictions and the actions are then used to estimate the Q-values using the neural network shown in Figure 4.3.

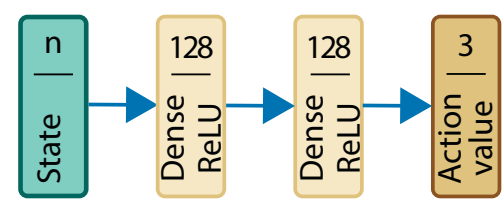

Figure 4.3: Architecture of the Q-net.

As shown in [78], the state representation network should not be updated with the same frequency of the Reinforcement Learning network due to the increase in the instability of the learning process. Therefore, we normally train the Qnet (Figure 4.3) at each training step, while we update only after a fixed number of training episodes the State-Net (Figure 4.2). The frequency of the update of the State-Net is chosen to be a trade-off between training too often and generate instability and training too rarely and slow down the learning of the optimal policy. 
Remember that, the optimal policy cannot be learned without an informative state representation.

In the episodes right after the updates of the State-Net, the rewards achieved by the Reinforcement Learning agent may drop due to the sudden changes of the state representation. To avoid learning local optimal policies, we hold constant the value of the $\epsilon$ of the $\epsilon$-greedy exploration policy of DQN throughout the whole training episodes.

\subsection{Experimental Design}

\subsubsection{Mobile Robot Navigation with Camera and LIDAR}

When autonomously navigating, mobile robots are usually equipped with multiple sensors in order to be able to gather the highest amount of information in the world and make possible decision-making. Commonly used sensors for perceiving the world are RGB cameras and laser range scanners (LiDARs). Therefore, we equipped our robot (TurtleBot 3 Waffle Pi) with a camera (FOV 60 ${ }^{\circ}$ ) and a $2 \mathrm{D}$ LiDAR (FOV $360^{\circ}$ ). The approach is first tested in different virtual environments (see Figure 4.4), simulated with Robot Operating System (ROS) and Gazebo, and later evaluated on the real robot (again TurtleBot 3 Waffle Pi).

\subsubsection{Reinforcement Learning Algorithm Settings}

The algorithm chosen is DQN with inputs the state predictions from the StateNet and with output the Q-values, one estimate per action. The agent can choose among 3 discrete actions: respectively, move forward, turn right, and turn left. To study the effect of different reward functions on the state representation learned with the new set of priors (Equation (4.1)-(4.4)), two different reward functions are employed for solving the different navigation tasks:

- Reward function based on the distance between the robot and the target:

$$
\mathrm{R}(s, a)= \begin{cases}r_{\text {reached }}, & d \leq d_{\text {min }} \\ r_{\text {crashed }}, & s=s_{t s} \\ 1-e^{\eta_{1} d}, & \text { otherwise. }\end{cases}
$$


- Reward function based on the orientation of the robot with respect to the target:

$$
\mathrm{R}(s, a)= \begin{cases}r_{\text {reached }}, & d \leq d_{\text {min }} \\ r_{\text {crashed }}, & s=s_{t s} \\ 1-e^{\eta_{2} \theta}, & \text { otherwise }\end{cases}
$$

where $d$ is the distance of the robot to the target, estimated using the odometry information, $\theta$ is the robot orientation with respect to the target, $d_{\min }$ is the minimum distance threshold below which the navigation target is considered reached and $\eta_{1}$ and $\eta_{2}$ are scaling factors for the exponential functions. $r_{\text {reached }}$ and $r_{\text {crashed }}$ are respectively a bonus for reaching the target and a penalty for hitting an obstacle, i.e. a terminal state $s_{t s}$. These two reward functions are common choices for solving robots navigation tasks.

\subsubsection{Navigation Tasks in Different Environments}

We first compare the new priors with the ones from [63] in order to highlight similarities and differences in the environment in Figure 4.4a. We then analyse the choice of the state dimensionality as being a crucial aspect of the RL performances. Furthermore, we study, through Principal Components Analysis, or PCA, [79] and t-Distributed Stochastic Neighbor Embedding, or t-SNE, [80] $]^{4}$, if the State-Net trained with the priors (Equation (4.1)-(4.4)) succeeds in encoding the meaningful information for solving the navigation task. In the case of the mobile robot navigation tasks proposed, this information corresponds to the world's physical properties such as, for example, the pose of the robot ( $x$-position, $y$-position, and $\theta$ orientation) and its distance to the target. Eventually, we test our approach in environments with different topologies and features (e.g. different colours of the wall or textures), shown in Figure 4.4b-4.4e, to validate the method. We also again study the information encoded by that State-Net and to what extent these are dependent on the environment topology.

\subsubsection{Multi-targets State Representation}

We perform experiments to evaluate the priors in a more complicated task: learning a state representation for multiple navigation targets. During training, at each

\footnotetext{
${ }^{4}$ These two techniques for dimensionality reduction allow us to visualise high-dimensional data clusters in a 2-dimensional space, understand and explain the learned state representation.
} 


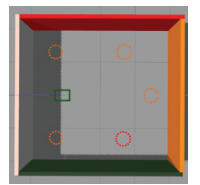

(a) Env-1

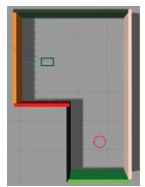

(b) Env-2

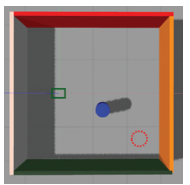

(c) Env-3

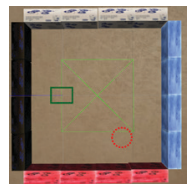

(d) $E n v-4$

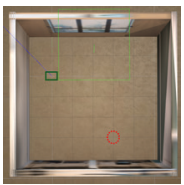

(e) Env-5

Figure 4.4: Simulation environments. The robot starting position is highlight by green rectangles, while possible targets locations by red circles.

episode, a target is sampled from a uniform distribution. We slightly adapt the observation vector to include the location $\left(x_{g}, y_{g}\right.$ coordinates) of the target. This information is directly passed to the last dense layer of the State-Net.

\subsubsection{Transfer Learning Experiments}

Transfer learning is an essential element for deploying RL algorithms on real robots, but it is usually limited by the simulation-reality gap, i.e. the difference between virtual simulations and the real world. However, we argue that, if informative high-level features are extracted from the observations, the Reinforcement Learning policies, trained on these features, gain robustness and can be transferred from simulation to real without any undesirable training on the real robots.

\subsection{Results and Discussion}

\subsubsection{Mobile Robot Navigation with Camera and LiDAR}

Here, we analyse the influence of the different sensor modalities on the learned representation. In particular, we compare the quality of learned representation through the crash ratio and the total cumulative reward when camera and LiDAR are used (Figure 4.5a and 4.5b), only the camera is used (Figure 4.5a and 4.5b) and only the LiDAR is used (Figure $4.5 \mathrm{a}$ and $4.5 \mathrm{~b}$ ). When both sensors are employed, the crash ratio is reduced (see Figure 4.5a) and the convergence speed is improved (see Figure 4.5b). This aspect shows how the representation learned with the priors can fuse the different sensor modalities to obtain the best set of features.

The complete video of the experiments in the simulation environment can be found at: https://youtu.be/AdjltfA0_GQ. 


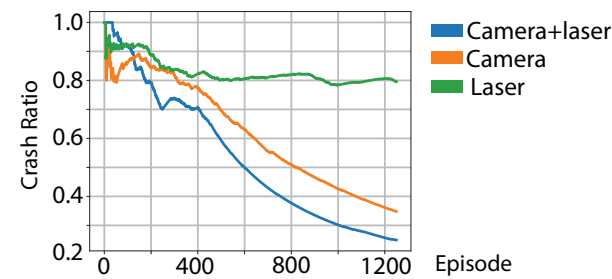

(a)

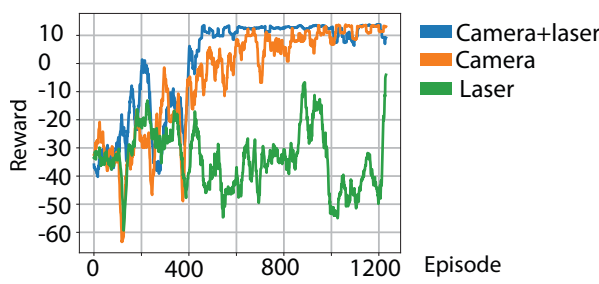

(b)

Figure 4.5: Crash ratio (4.5a) and cumulative reward (4.5b) obtained during training using different sensor modalities.

\subsubsection{Navigation Tasks in Different Environments}

\section{Comparison with Original Priors}

The comparison with the priors, introduced in [63], is performed by comparing the effect of the different learned state representations on the performance of the Reinforcement Learning agent in the environment depicted in Figure 4.4a. For the sake of a fair comparison, the training and testing environment is similar to the one used in [63]. Furthermore, the same neural networks and hyperparameters are used. In Figure 4.6, the crash ratio during training is shown when the proposed priors and the original priors are used. As shown in Figure 4.6, the new set of

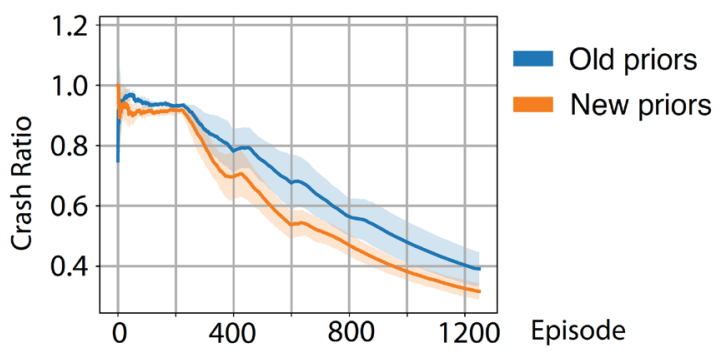

Figure 4.6: Crash ratio when the new priors and the original priors are used on the same navigation task.

priors can improve the agent's performances by reducing the average crashing ratio and its variance during training. This improvement occurs because the new priors enforce a state representation that better incorporates the properties of the reward function that has to be maximised by the Reinforcement Learning algorithm. This is further elaborated on in Section 4.4.2. 


\section{Analysis of the State Dimensionality}

The choice of the state dimensionality is crucial for the performance of the Reinforcement Learning algorithm. To validate this claim, we analyse the crash ratio, i.e. the number of times an episode ends due to a collision with an obstacle over the total number of episodes, in relation to the choice of the state dimension. This choice corresponds to the choice of the output dimension of the State-Net. The results are shown in Figure 4.7. It is possible to notice that if the state dimension

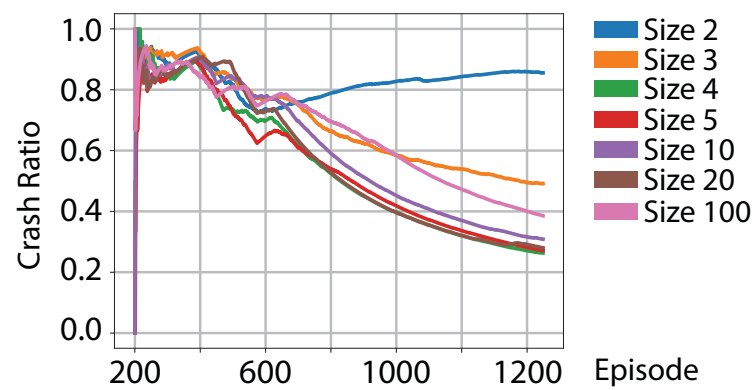

Figure 4.7: Crash ratio of the agent with respect to the state dimension.

is chosen too small with respect to the optimal one, the encoding step loses much important information due to the excessive compression. This is the case for the state dimension equal to two and three. In those cases, the agent struggles to reduce the collisions and, consequently, to improve the policy. On the other hand, if the dimension is chosen too big, for example, equal to hundred, the agent's performances are slowed down due to the lack of compression and, to some extent, to the curse of dimensionality. The agent, in this case, has to learn which information has to be ignored.

We compare our approach with DQN using the true pose of the robot, $x$ position, $y$-position and $\theta$ orientation, and end-to-end DQN using the raw observations directly (see Figure 4.8) in the environment in Figure 4.4a. As expected, the agent observing the true state of the environment quickly learns the optimal solution (blue line in Figure 4.8). However, the knowledge of the true environment state is a limiting factor in many real-world scenarios. When the state representation, learned with the State-Net and the proposed priors, is combined with the DQN (orange line in Figure 4.8) after a few updates of the State-Net, occurring at episode 200 and 400 respectively, the policy converges to the optimal solution 


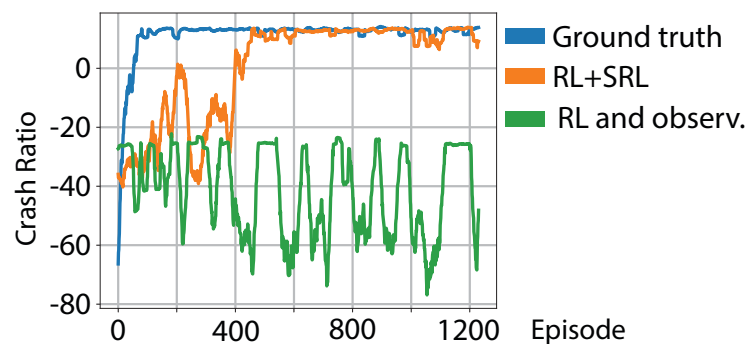

Figure 4.8: Comparison of RL based on true pose, SRL combined with RL (ours) and $\mathrm{RL}$ with input the raw observations.

with a convergence rate very similar to the one using the true state of the environment. The policy learned directly on the raw observations (green line in Figure 4.8) cannot converge in the time window of 1200 episodes. This result proves the effectiveness of the state representation learning using the priors. Compared to the end-to-end approach, splitting the feature learning with the policy learning greatly increases the sample efficiency of the Reinforcement Learning agent.

Through PCA, we study the actual dimensionality of the encoded state space by counting the number of uncorrelated components. The method is tested for all the environments in Figure 4.4. The results obtained are shown in Table 4.1. The

\begin{tabular}{||c|c|c||}
\hline Environment & State-Net output dim & Nr. uncorr. components \\
\hline \hline Env-1 & 10 & 4 \\
Env-2 & 10 & 4 \\
Env-3 & 10 & 5 \\
Env-4 & 10 & 4 \\
Env-5 & 10 & 4 \\
\hline
\end{tabular}

Table 4.1: Analysis of the state predictions dimensions in the different environments.

state representation learned with the priors is not dependent on the topology of the environment (e.g. its shape) or the choice of the features (e.g. walls' colors or textures) as the number of uncorrelated component is consistently 4 in Env-1, Env-2, Env-4 and Env-5 (see Table 4.1). This proves that the state representation learning method proposed generalises well in different environments. Interestingly, in Env-3, when an obstacle is present on the optimal trajectory towards the target, the state representation can encode that information. This aspect is reflected in the number of uncorrelated components, as a fifth one emerges. This again proves 
that the state representation learned with the new priors can encode the taskrelevant information.

In order to understand what kind of information the State-Net encodes in the learned state space, we compare samples from the different principal components with the important physical properties required in any navigation task: pose of the robot $(x, y, \theta)$ and its distance to the target. The results of the correlation analysis, for the environment in Figure 4.4a, are shown in Table 4.2. A correlation

\begin{tabular}{||l|c|c|c|c||}
\hline & x-position & y-position & orientation & distance to target \\
\hline \hline Principal component 1 & 0.86 & 0.24 & 0.18 & -0.14 \\
Principal component 2 & -0.28 & 0.68 & 0.7 & 0.8 \\
Principal component 3 & -0.32 & -0.17 & -0.37 & 0.22 \\
Principal component 4 & -0.1 & 0.19 & -0.09 & 0.13 \\
\hline
\end{tabular}

Table 4.2: Correlation analysis of the principal components and the physical properties.

exists between the true physical properties of the world and the encoded properties by the State-Net. It is worth mentioning that we are not enforcing any explicit disentanglement and independence of the state properties, as we are still in a self-supervised learning framework.

\section{Reward-shaped State Representation}

To test if the reward signal, combined with the new priors, can be used to effectively shape the state representation by encoding from the sensors information taskspecific knowledge, we analyse, using t-SNE, the state representations obtained when the different reward functions, in Equation (4.6) and (4.7), are employed. In particular, we analyse if the clustering of the state predictions is related to the chosen reward function and, consequently, to the important properties of the problem. In Figure 4.9, the clustering of the state predictions, when the reward function in Equation (4.6) is used, with respect to the true distance from the target (see Figure 4.9a) and orientation from the target (see Figure 4.9b) is shown. It is possible to notice that when the reward function in Equation (4.6) is used, the state representation can encode and cluster close together the predictions that have similar rewards, i.e. similar distance from the target. When the same predictions are overlapped with the true orientation (see Figure 4.9b), the clustering is less effective, and the prediction samples with similar orientation are spread over larger 

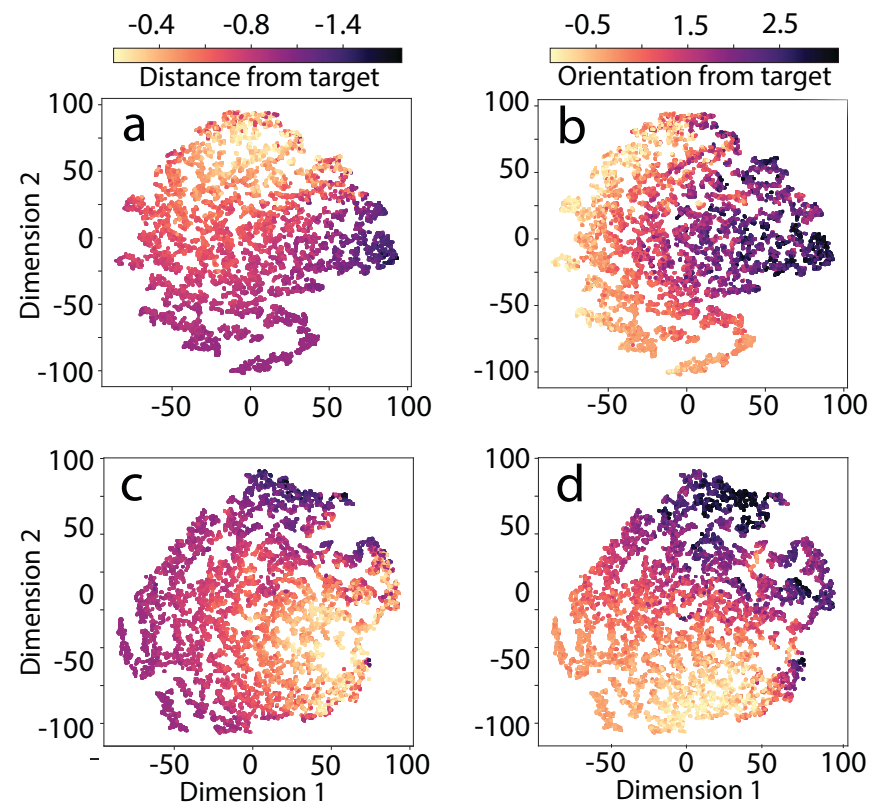

Figure 4.9: Distance-based reward (a and b) and orientation-based reward (c and d). Distance vs orientation clustering (t-SNE visualisation).

areas. This is expected since the state representation is not trained to cluster the predictions with respect to the orientation. Analogously, the clustering of the state predictions when the reward function in Equation (4.7) is used, with respect to the true distance from the target (see Figure 4.9c) and orientation (see Figure $4.9 \mathrm{~d}$ ) is shown. When the reward function in Equation (4.7) is used, the predictions are correctly clustered with respect to the true orientation (see Figure 4.9d), but also with respect to the true distance (Figure 4.9c). This is because the orientation with respect to the target is computed using the distance from the target along the $\mathrm{x}$ and $\mathrm{y}$-axis. Thus, it is not completely independent of the distance. These results prove that the state representation encodes task-relevant knowledge through the reward information.

\subsubsection{Multi-target Navigation}

Here, we present the results related to multi-target navigation. In particular, we analyse if the priors are suitable for learning a state representation capable of differentiating between multiple navigation targets (two in this case). The results 
are presented in Figure 4.10, where the state predictions are again analysed using PCA (Figure 4.10a) and t-SNE (Figure 4.10b) . The state representation learned can effectively incorporate the information of the different targets. It can cluster the learned state space not only in terms of the reward in Equation (4.6) (this can be noticed by looking at the smoothness of the colour gradient in the Figures) but also with respect to the two targets (a clear division of the state samples).

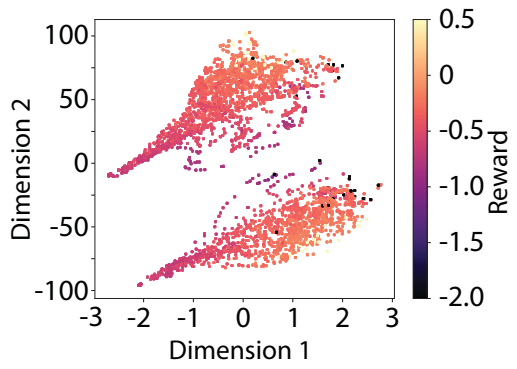

(a)

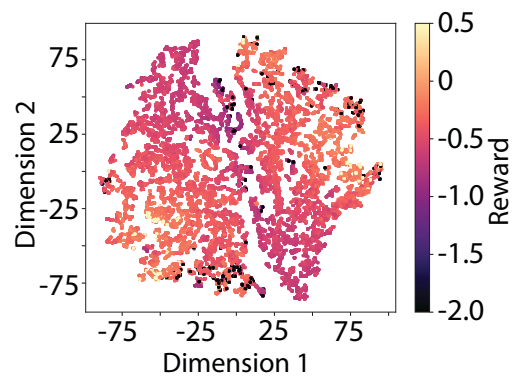

(b)

Figure 4.10: State representation learned for two different targets analysed with PCA (4.10a) and t-SNE (Figure 4.10b).

\subsubsection{Experiments in a Realistic Simulation Environment and on a Real Robot}

Eventually, the transfer learning experiments are presented. In particular, we show, for a single navigation target, the trajectories followed by the real robot after transferring the state representation and the policy learned in the simulation environment $4.4 \mathrm{~d}$. The trajectories followed on ten different experiments, are shown in Figure 4.11a. To assess the robustness of the state representation and the policy learned in simulation to variations in the sensor reading, during the experiments, we switched off the lights of the room, and after few seconds, we switched them back on. In Figure 4.11a, the trajectories obtained are shown. When the lights are off, the agent receives images from the camera which are very different from the one it has been trained on. Therefore, it cannot immediately find the key features to reach the target. However, the agent does not perform random actions that would bring the robot to crash against an obstacle (purple dots in Figure 4.11a). The agent starts a searching behaviour as it rotates around 
in search of the correct features. Once the light is turned on again (blue dots in Figure 4.11a), the agent quickly recognizes the features and drives safely to the target. This result can be interpreted as proof that the policy has learned robust obstacle avoidance and navigation skills.

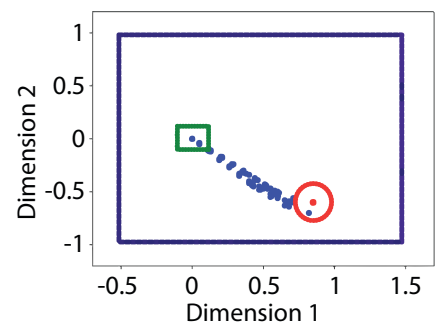

(a)

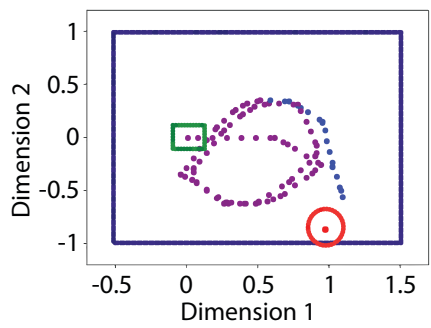

(b)

Figure 4.11: Trajectories of the real robot (x-y plane) when the light is on (left). The green rectangle corresponds to the starting location of the robot, while the red circle corresponds to the target location. The blue dots are the trajectories samples. Trajectories of the real robot when the light is off (right) indicated with purple dots and when the light is turned on again (blue dots).

By extracting the meaningful features from the sensor data using the State-Net, not only the agent learns the policy faster, but we can mitigate the simulation to reality gap, and we can directly transfer the knowledge learned in the simulation environment to the real robot without any further training on the real hardware.

The video of our real-world experiments can be found at: https://youtu.be/ mvQcxZ9tVZk.

\subsection{Conclusions}

This chapter proposes a new approach for the self-supervised learning of state representations for Reinforcement Learning. The state representation is learned using a new set of auxiliary loss functions, i.e. the reward-shaped priors. These priors are shaped using the reward function to incorporate the task-relevant knowledge in the state representation. From the tests on the different environments, the state representation, built using the reward-shaped priors, can encode the essential physical properties for solving different navigation tasks. Furthermore, the state representation learned is not dependent on the topology of the environment or the textures in it. The number of uncorrelated components in the state is consistently 
equal to four. However, when an extra constraint is added in the environment, e.g. an obstacle (see Figure 4.4c), the state representation grows an extra uncorrelated component to encode information about the obstacle. The same happens in the case of multi-target navigation tasks. Furthermore, the priors allow the fusion of different sensor modalities, camera and LiDAR in this case. Eventually, the state representation and policy learned in the simulation environment are successfully transferred to the real robot without further retraining with real-world data. 



\section{Chapter 5}

\section{Low-Dimensional State Representation Learning with Robotics Priors in Continuous Action Spaces}

Reinforcement Learning algorithms have proven to be capable of solving complicated robotics tasks in an end-to-end fashion without any need for hand-crafted features or policies. Especially in the context of robotics, in which the cost of realworld data is usually extremely high, Reinforcement Learning solutions achieving high sample efficiency are needed. In this paper, we propose a framework combining the learning of a low-dimensional state representation, from high-dimensional observations coming from the robot's raw sensory readings, with the learning of the optimal policy, given the learned state representation. We evaluate our framework in the context of mobile robot navigation in the case of continuous state and action spaces. Moreover, we study the problem of transferring what learned in the simulated virtual environment to the real robot without further retraining using real-world data in the presence of visual and depth distractors, such as lighting changes and moving obstacles. A video of our experiments can be found at: https : //youtu. be/rUdGPKr2Wuo.

This chapter includes an adaptation of:

Nicolò Botteghi, Khaled Mustafa, Mannes Poel, Beril Sirmacek, Christoph Brune, Abeje Mersha, and Stefano Stramigioli. Low-Dimensional State Representation Learning with Robotics Priors in Continuous Action Spaces. International Conference on Intelligent Robots and Systems, 2021.

The Section Background was removed as treated in Chapter 2 and 3, and the notation adapted to be consistent with the notation introduced in this thesis. 


\subsection{Introduction}

${ }^{1}$ Reinforcement Learning has shown to be able to learn complex behaviours directly from high-dimensional input data, e.g. raw pixel inputs, in different domains such as robotics [50], [51] and video-games [40], [48], [49]. Thus, Reinforcement Learning has the potential to achieve high degrees of motoric and cognitive intelligence.

Learning from high-dimensional inputs, or observations, comes at the price of low sample efficiency and high computational load. The problem of high sampleefficiency of the algorithms is of crucial importance in robotics due to the cost of real hardware and simulators [10], [81]. Unfortunately, simulators rely on mathematical models (e.g. physics) that approximate the real world. These approximations make transfer learning a big challenge in many cases. This problem is referred to, in literature, as the simulation-to-reality gap [10]. Moreover, when aiming to solve real-world challenges, we must rely on raw sensory data, such as RGB cameras or LIDARs sensors. Sensory data are often noisy, partial and high-dimensional, and therefore it is difficult to deal with them.

The state space can be manually hand-crafted to contain only the relevant features and consequently reduce its dimensionality to overcome the issue of learning policies directly from raw observations and improve sample efficiency. While this step is possible for simple tasks, designing the state space is a non-trivial challenge in real-world problems. Moreover, hand-crafted features are usually brittle to changes in environments such as lighting conditions or non-modelled dynamics. The robustness of feature extraction methods is especially critical in robotics Reinforcement Learning that heavily relies on virtual simulators to reduce the cost of real-world samples. Therefore, we need methods for extracting task-relevant features in robust and sample-efficient ways.

State representation learning [6] is the name given to all the methods learning to extract informative and compact state representations from high-dimensional observations to facilitate the learning of the policy of the Reinforcement Learning algorithms. Instead of directly solving the problem of learning the optimal policy, mapping observations to actions, it is beneficial, for sample-efficiency and robustness of the learned policy, to first learn a low-dimensional representation of the

\footnotetext{
${ }^{1}$ Chapter 4 and Chapter 5 study the problem of state representation learning using the robotics priors (see Section 3.3.1). However, while the method proposed in Chapter 4 utilises the reward samples, in this chapter, we use the actions of the agent sampled from a continuous action space to improve the quality of the learned representation.
} 
observations, i.e. the state representation, then learn the optimal policy directly from such representation.

Robots live in spaces governed by physical laws. These spaces have a lower dimensionality than the space of raw sensory observations. We can exploit such knowledge and represent the sensory information into learned state spaces of low dimensionality. Moreover, additional and general priors [77], i.e. prior knowledge, of the world can be used to aid the learning of meaningful state representations, such as smoothness of the state changes or the relation between action magnitude and state changes. These priors can be used to shape the loss functions used for learning such mappings. In the particular case of robotics, the authors of [63] propose the so-called robotics priors, i.e. loss functions that are shaped to incorporate physical knowledge of the world and used to constrain and steer the learning of the state representation loosely. The robotics priors are means to learn meaningful state representations in all the cases in which physical laws govern the environment, and they consequently allow robustness and sample efficient learning of the Reinforcement Learning policy.

This research proposes a framework for state representation learning and Reinforcement Learning to efficiently solve mobile robots navigation tasks directly from raw sensory information. This framework is shown in Figure 5.1. Our re-

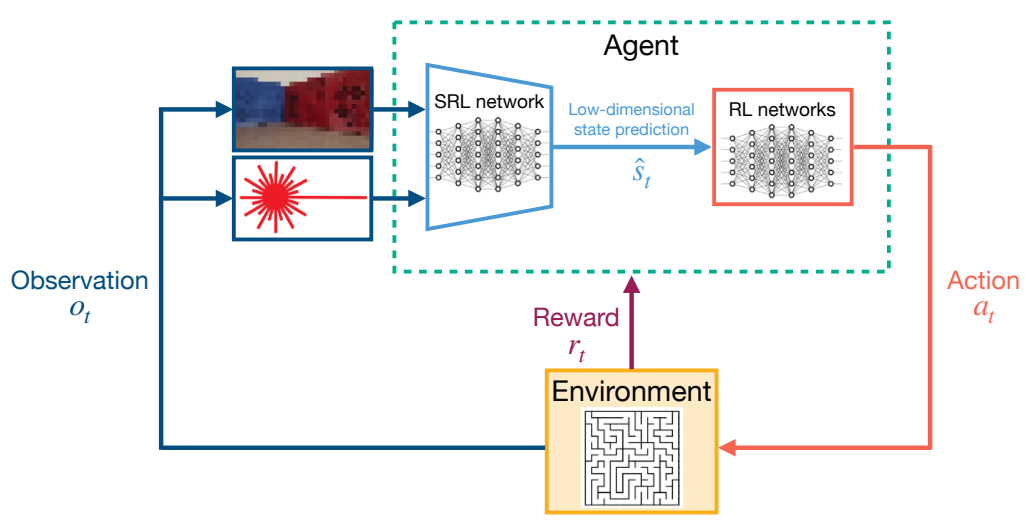

Figure 5.1: Proposed framework combining state representation learning and Reinforcement Learning. 
search contributes to improving the sample efficiency of the Reinforcement Learning algorithm, increasing robustness and interpretability of the learned state features, combining the use of deep learning with prior knowledge, and bridging the simulation-to-reality gap. In particular:

- We study the problem of unsupervised learning, i.e. without the need for expensive labelled data, of meaningful and interpretable state representations using the robotics priors in continuous state and action spaces. Continuous action spaces allow smoother and more advanced trajectories, and therefore should be preferred for robotics.

- We introduce a new set of robotics priors, i.e. loss functions for learning the state representation, that exploits the underlying structure of continuous action spaces for obtaining smoother and more informative state representations. The new loss functions do not only contain information of states but also actions.

- Eventually, we show that the proposed framework allows the transfer of the policies and representations learned in virtual simulation environments to the real robot without further retraining using real-world data.

\subsection{Related Work}

The concept of robotics priors is introduced by [63] for solving the problem of unsupervised learning of an informative state representation in the context of simple robotics navigation tasks. In those experiments, the agent's action space is chosen to be discrete, and the mobile robot can only move forward, backward, left or right at each time step. Furthermore, the robot is equipped with an RGB camera with a field of a view of $300^{\circ}$.

In [12], the priors are adapted to incorporate the reward properties better, improve the quality of the learned state representation and, consequently, the quality of the learned Reinforcement Learning policy.

In [64], the authors propose the so-called position-velocity encoder for learning a valid state representation. This work introduced new prior losses that exploit the relation between the position and the velocity of the inverted pendulum, the 
cart-pole and the ball-in-a-cup problems. Using the position-velocity encoder, all these different tasks can be efficiently learned from pixel inputs.

In [82], the original set of robotics priors [63] is evaluated in the presence of distractors, i.e. disturbing visual elements such as shadows or randomly moving backgrounds. When the domain randomisation is strong, the robotic priors struggle to construct a coherent state representation. Therefore, the authors propose the reference-point prior, using true state values, to regularize the learned state space and mitigate such a limitation.

Eventually, in [83], the authors propose an approach for learning state representation using the robotics priors in the case of more complex environments where a single observation is not enough to distinguish between two or more states. Therefore, they employ a recurrent LSTM-based encoder, mapping sequences of observations to single state predictions. Moreover, to obtain a coherent representation, they extend the reference-points prior, proposed in [82], to include multiple reference points, or landmarks, to connect the state predictions from different trajectories.

Differently from all these approaches, we study the problem of learning state representations in the context of continuous action spaces and by exploiting the underlying action structure when constructing the priors, i.e. the loss functions. Here, we do not consider heavy domain randomisation nor the problem of a recurrent state representation. However, we focus on a purely unsupervised approach that does not require the knowledge of true state values. Our approach can be considered orthogonal to [82] and [83]. The proposed priors can be directly combined with the reference-points prior and with a recurrent encoder network. Moreover, differently from [64], [82], [83], we test our approach on a real robot.

Eventually, all these approaches study the problem of state representation learning by introducing different robotics priors with the underlying assumption of discrete action spaces. In our work, we study the problem of state representation learning in continuous action spaces. Moreover, we aim at exploiting the structure of the action space to improve the quality of the learned state representation and, consequently, the efficiency of the policy learning. 


\subsection{Methodology}

\subsubsection{Proposed Approach}

We propose a framework for sample efficient end-to-end RL for solving different robotics navigation tasks directly from high-dimensional sensory readings. The framework is composed of two building blocks: first, a low-dimensional state representation is learned from the observations, and then the optimal policy is learned solely based on such state representation, as shown in Figure 5.1.

The state representation is learned in an unsupervised fashion by introducing a new set of robotics priors. The robotics priors are a way to inject prior knowledge into the state representation learning step by loosely constrain the learned state space. Our work extends the concept of robotic priors to continuous action spaces by exploiting the underlying structure of the actions into the encoding step of the state representation learning.

For learning the optimal policy, we choose DDPG (see Section 3.2.2) as the candidate RL algorithm for its ability to deal with continuous state and action spaces. However, it is worth mentioning that the proposed approach is independent of the specific RL algorithm and any algorithm that can deal with continuous action space can be in principle employed.

With this work, we aim at addressing the following research questions:

- How can we take advantage of the underlying action structure of continuous action spaces for learning the state representation using the robotic priors?

- To what extent can the priors, exploiting actions structure, be beneficial for policy learning?

- To what extent can the learned state representations, using the proposed priors, and the policies learned from such representations be transferred to real robots without further re-training using real-world data?

\subsubsection{Robotics Priors for Continuous Action Spaces}

Continuous actions spaces have an underlying structure that we can exploit when learning a low-dimensional state representation. In many situations, observation changes and, consequently, agent's state changes are directly related to the magnitude of the action taken. This simple concept can be exploited and used as a loose 
constraint when learning the state representation using the robotics priors. The new set of robotics priors is presented below, where $\hat{s}_{t}=\phi_{e}\left(o_{t} ; \boldsymbol{\theta}_{e}\right)$ corresponds to the state prediction of the encoder neural network $\phi_{e}$, parametrised by $\boldsymbol{\theta}_{e}$, given observation $o_{t}, a_{t}$ is the action and $\Delta \hat{s}_{t}=\hat{s}_{t+1}-\hat{s}_{t}$.

Simplicity prior: The task-relevant information lies in a space with dimensionality much smaller than the sensory observations.

Temporal coherence prior (new): The temporal coherence prior, introduced in [63], encodes the property that state changes are slow and states close in time should also be close in space. However, this prior treats all the state pairs equally and does not consider the magnitude of the action taken. In our approach, we use the magnitude of the action $a_{t}$, connecting state prediction $\hat{s}_{t}$ and next state prediction $\hat{s}_{t+1}$, as weighting factor for the state distance $\left\|\Delta \hat{s}_{t}\right\|$. In particular, when an action with a large magnitude connects two state predictions, the loss function does not enforce the states to be as close as when an action with a small magnitude is taken. In this way, we can better exploit the structure of the smooth and continuous action space. This intuition leads to the prior in Equation (5.1).

$$
\mathcal{L}_{\text {temp }}\left(\boldsymbol{\theta}_{e}\right)=\mathbb{E}\left[\left(\left\|\Delta \hat{s}_{t}\right\| e^{-\alpha\left\|a_{t}\right\|}\right)^{2}\right]
$$

where the hyper-parameter $\alpha$ is used to weight the effect of the action magnitude on the state difference.

Proportionality prior (new): The original proportionality prior [63] encodes the heuristic that the state variation of two different state pairs should be similar if the actions taken are similar. With similar reasoning to the case of the temporal coherence prior, in continuous action spaces, the similarity in states property translates into an additional weighting factor dependent on the difference in magnitude between the actions $a_{t_{1}}$ and $a_{t_{2}}$. The action difference scales the need of minimizing the state difference. The new proportionality prior is shown in Equation (5.2).

$$
\mathcal{L}_{\text {prop }}\left(\boldsymbol{\theta}_{e}\right)=\mathbb{E}\left[\left(\left\|\Delta \hat{s}_{t_{2}}\right\|-\left\|\Delta \hat{s}_{t_{1}}\right\|\right)^{2} e^{-\beta\left\|a_{t_{1}}-a_{t_{2}}\right\|^{2}}\right]
$$

where hyper-parameter $\beta$ is used to weight the effect of the action difference on the state difference.

Repeatability prior (new): The repeatability prior reinforces the similarity of states not only in magnitude but also in direction. In particular, if two different 
states are similar and similar actions are taken in each of them, the magnitude of difference in the transition $\Delta s_{t_{1}}$ and $\Delta s_{t_{2}}$ should be limited. The new repeatability is shown in Equation (5.3).

$$
\mathcal{L}_{\text {rep }}\left(\boldsymbol{\theta}_{e}\right)=\mathbb{E}\left[\left\|\Delta \hat{s}_{t_{2}}-\Delta \hat{s}_{t_{1}}\right\|^{2} e^{-\left\|\hat{s}_{t_{2}}-\hat{s}_{t_{1}}\right\|^{2}} e^{-\beta\left\|a_{t_{1}}-a_{t_{2}}\right\|^{2}}\right]
$$

Causality prior (new): The temporal coherence and proportionality are aggregating priors that tend to reduce the distance between the state predictions. Therefore, to prevent the trivial mapping in which all the states are mapped to the origin, we employ a contrastive loss pushing apart state predictions. We further enhance the so-called causality prior, with an additional penalty dependent on the action difference. This prior is shown in Equation (5.4).

$$
\mathcal{L}_{\text {caus }}\left(\boldsymbol{\theta}_{e}\right)=\mathbb{E}\left[e^{-\left\|\hat{s}_{t_{2}}-\hat{s}_{t_{1}}\right\|^{2}} e^{-\beta\left\|a_{t_{1}}-a_{t_{2}}\right\|^{2}}\right]
$$

Total loss: The overall loss function that is minimised for learning the state representation is shown in Equation (5.5).

$$
\min _{\boldsymbol{\theta}_{e}} \mathcal{L}\left(\boldsymbol{\theta}_{e}\right)=\omega_{1} \mathcal{L}_{\text {temp }}\left(\boldsymbol{\theta}_{e}\right)+\omega_{2} \mathcal{L}_{\text {prop }}\left(\boldsymbol{\theta}_{e}\right)+\omega_{3} \mathcal{L}_{\text {rep }}\left(\boldsymbol{\theta}_{e}\right)+\omega_{4} \mathcal{L}_{\text {caus }}+\omega_{5} \mathcal{L}_{\text {reg }}\left(\boldsymbol{\theta}_{e}\right)
$$

where $\omega_{1}=1, \omega_{2}=1, \omega_{3}=1, \omega_{4}=2, \omega_{5}=1$ and $L_{\text {reg }}$ corresponds to the $L_{2}$ regularization loss. The weights of the single loss functions are chosen by performing a grid search $\in\{1,2,3,4,5\}$. However, the method is not highly sensible to the choice of the hyperparameters and other sets of weights may produce similar results.

\subsubsection{Neural Network Architectures}

As shown in Figure 5.1, the framework includes two mappings: from observations to low-dimensional state predictions and from state predictions to actions. Both mappings are learned through neural networks. In particular, the SRL network, in Figure 5.2, is responsible for the learning of the low-dimensional state representation from the multi-modal sensory observations. The architecture is similar to the one used in [12], where the sensor modalities are independently processed by convolutional layers, flattened and merged through fully-connected layers to 


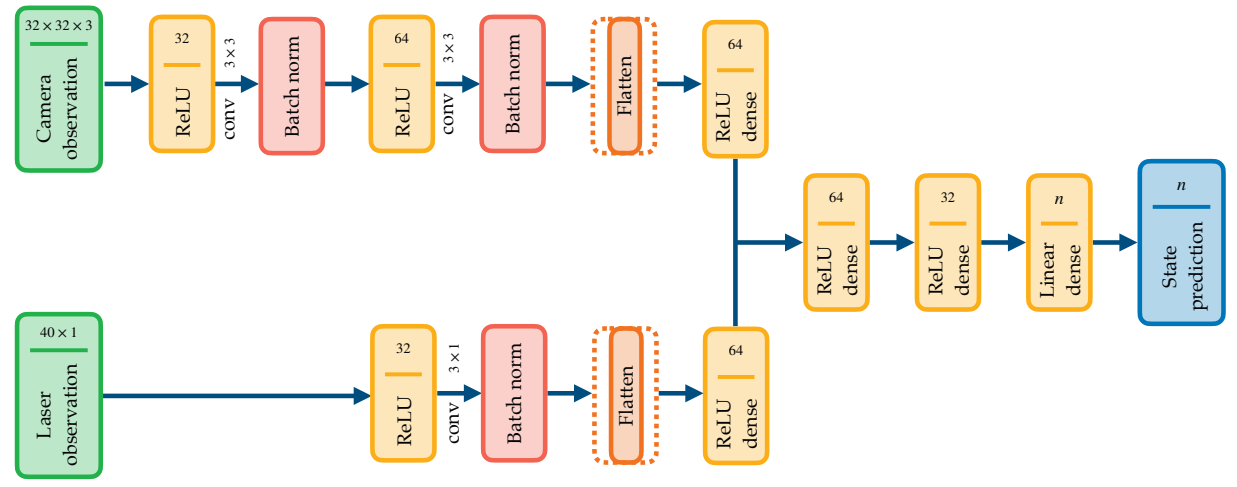

Figure 5.2: Architecture of the SRL network.

create the final low-dimensional state prediction of dimension five. The state dimension is chosen accordingly to the studies done in [12] and [83]. On the other side, the $R L$ networks, shown in Figure 5.3, composed of an actor mapping states to actions, and a critic estimating the action-value function, are responsible for the learning of the optimal policy. Both architectures present three fully-connected hidden layers of dimension 512. The output layer of the actor generates the linear and angular velocities set-point for the low-level controllers of the robot. We allow only forward motion by limiting the linear velocity with sigmoid activation. The output of the critic estimates the Q-value of the input state-action pair.

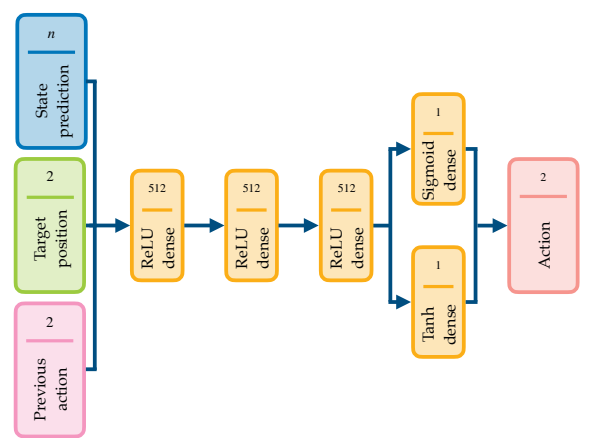

(a) Actor network.

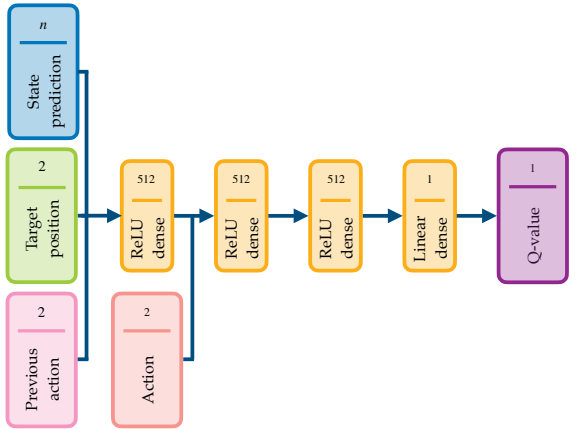

(b) Critic network.

Figure 5.3: Architecture of the RL networks. 


\subsection{Experimental Design}

\subsubsection{Mobile Robot Navigation with Multiple Sensor Modal- ities in Different Environments}

We evaluate the proposed approach by studying the problem of learning to control a differential-drive mobile robot, equipped with an RGB camera (images of size $32 \times 24 \times 3$ ) and a $2 \mathrm{D} \mathrm{LiDAR}\left(40\right.$ data points on the $360^{\circ}$ range), to reach various target locations without collision with obstacles in different environments. Examples of environments are shown in Figure 5.4.

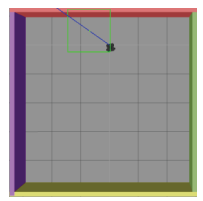

(a) $E n v-1$

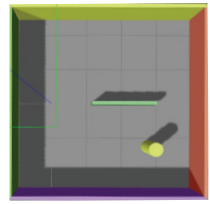

(b) Env-2

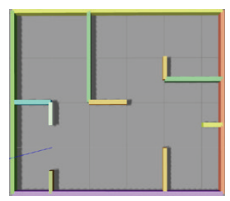

(c) Env-3

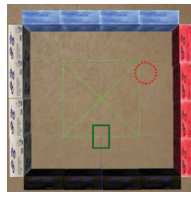

(d) $E n v-4$

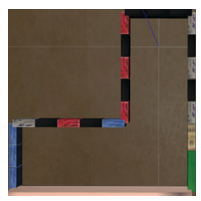

(e) $E n v-5$

Figure 5.4: Examples of simulation environments.

Since investigating the applicability of transfer learning is one of the goals of this work, we first experiment in virtual simulated environments using the ROSGazebo platform, where we aim at learning the state representation and the optimal policy. Then, we transfer the learned models to the real robot without further training using real data. For the transfer learning experiments, we use the environments depicted in Figure 5.4d and 5.4e. The robot used is the TurtleBot 3 Waffle Pi.

For all the experiments, we use a distance-based reward function, as shown in Equation (5.6).

$$
\mathrm{R}\left(s_{t}, a_{t}\right)= \begin{cases}r_{\text {reached }}, & d \leq d_{\text {min }}, \\ r_{\text {crashed }}, & s_{t}=s_{t s} \\ -\zeta\left(d_{t}-d_{t-1}\right), & \text { otherwise. }\end{cases}
$$

where $d_{t}=\left\|p_{t}^{x, y}-g\right\|_{2}$ is the distance from the current position $p_{t}$ of the robot at time $t$ with respect to the inertial frame and the target's location $g$ expressed in the robot's coordinate frame, $d_{t-1}$ the distance at time $t-1, r_{\text {reached }}$ is a bonus for reaching the target, $d_{\min }$ is the minimum distance threshold below which the navigation target is considered reached, $\zeta$ is a scaling factor, and $r_{\text {crashed }}$ is a 
penalty for reaching a terminal state $s_{t s}$, e.g. hitting an obstacle or reaching the maximum number of steps in a single training episode. A distance-based reward function is a common choice for solving navigation tasks [84], [16].

\subsubsection{Baselines}

We compare the proposed approach for learning the state representation with:

- The adaptation of original robotics priors introduced in [63] for continuous action spaces, where the action equality is replaced with a similarity between the action pairs, i.e. two actions are similar if the difference of their magnitudes is below a given threshold.

- The reward-shaped robotics priors proposed in [12].

- The robotics priors proposed in [83], where, for fairness of the comparison, we remove the landmark prior that requires true state values.

- An Auto-Encoder (AE), where the latent code of the AE is used as an input to the Reinforcement Learning networks.

For each environment, we collect a data set comprised of randomly generated trajectories. We first learn a state representation of dimension five for each approach by updating the $S R L$ network for 20 epochs. In the AE case, the state dimension is set to 20, and the training lasts 200 epochs. Because our goal is to reach any possible target in a given environment, at each training episode, we randomly change the goal location and the starting pose of the robot to prevent getting biased by the environment structure. Therefore, to inform the agent of the target information, we concatenate the learned state predictions to the $(x, y)$-coordinates of the target position. Eventually, for further training stability, the previous action taken by the agent is also added to the complete extended state vector to improve the smoothness of the resulting trajectories.

We analyse the quality of the learned state representations and the consequent performances in terms of success ratio ${ }^{2}$ over the training of the RL agent when fed with the learned representations, mean Ground Truth Correlatetion (GTC) as in [85], mean K-Nearest Neighbour (KNN) as in [83], and mean Linear Reconstruction Error (LRE). For fairness of comparison, the same $S R L$ and $R L$ networks

\footnotetext{
${ }^{2}$ The success is defined by the robot reaching the target without collisions.
} 
architectures are used for each method, with the exception of the AE, where a decoder network is added to reconstruct the input data from the latent code.

Additionally, we compare with the agent trained using the true state, i.e. the pose of the robot. We concatenate the target position and the previous action taken for fairness of comparison to the true pose.

\subsection{Results and Discussions}

\subsubsection{Analysis of the Learned State Representations}

In Figure 5.5, we show, through the plotting of the true states, i.e. the true robot's poses, and the first two principal components computed using PCA [79], of the different state representations obtained when training the $S R L$ network with the different baselines in environment Env-1. Due to the additional regularisation provided by the action terms in the priors (see Equation (5.1)-(5.4)), the learned state space appears to be the smoothest and most coherent with the true state space. Our approach achieves the highest mean GTC and LRE compared to other methods and the third best KNN score, as shown in Table 5.1.

\begin{tabular}{||c|c|c|c||}
\hline Approach & mean GTC & mean KNN $(\mathrm{k}=20)$ & mean LRE \\
\hline \hline our & 0.7318 & 1.8207 & 1.6289 \\
priors in [63] & 0.6971 & 1.8029 & 1.7032 \\
priors in [12] & 0.7002 & 1.8305 & 1.7022 \\
priors in [83] & 0.6508 & 1.8283 & 1.6405 \\
AE & 0.6266 & 1.7862 & 1.6415 \\
\hline
\end{tabular}

Table 5.1: Quantitative evaluation of the different approaches in environment Env-1.

These results mean that our approach better incorporates the properties of the true state space ${ }^{3}$. In particular, the priors proposed in [12], in Figure 5.5c, suffer from a slight lack of smoothness near obstacles due to sudden changes in the rewards. The original priors, proposed in [63], in Figure 5.5d, by forcefully aggregating states in which similar actions are taken, disrupt the intrinsic smoothness of state and action spaces. The prior in [83] in Figure 5.5e, can achieve a good

\footnotetext{
${ }^{3}$ It is worth noticing that in the context of unsupervised representation learning without labelled data, the first and second dimension of the learned state space may not correspond necessarily to the $\mathrm{x}$-position and y-position, but they may appear flipped (see Figure 5.5b).
} 
representation, even though less smooth when compared to our priors especially around obstacles. By evaluation pairs of state predictions, our proportionality prior (in Equation 5.2) and repeatability prior (in Equation 5.3) better regularize all the transitions leading to a collision with obstacle, as it can be notice by the clustering on the collision states in Figure 5.5b. Eventually, as expected, the AE, in Figure 5.5f, learns the least interpretable representation. The latent states are only aggregated based on similarities of the observations, and there are no guarantees that two consecutive yet different observations are mapped close to each other.

\subsubsection{Simulation Results}

To assess the quality of the learned state representations in quantitative manners, we compare the performance of the different $\mathrm{RL}$ agents when trained on such representations. Figure 5.6 shows the success ratio over training of the agents for the different simulation environments depicted in Figure 5.4a-5.4c. The agent trained with the proposed method for state representation learning outperforms the other baselines ${ }^{4}$ due to the smoother and more coherent state representation that betters incorporates the properties of the true state space.

Furthermore, in Figure 5.7, we show the trajectories achieved after training by our approach. The robot has to reach a set of targets in Env-2 and Env-3 based on the sequence labelled in the Figure. The agent can successfully reach a sequence of different targets without collision with the obstacles.

\subsubsection{Real-World Experiments}

Eventually, we train the state representation and policy networks in the simulated environments Env-4 and Env-5, depicted in Figure 5.4d and 5.4e respectively, that resemble the real-world environments. Then, we directly transfer them to the real robot without further retraining using real-world data. The learned lowdimensional state representation can robustly extract the most important features out of the sensory readings, and it can effectively reduce, if not cancel out the simulation-to-reality gap. Moreover, we test the robustness of the learned model

\footnotetext{
${ }^{4}$ The state representation incorporates information about the robot's pose, the obstacles and the topology of the environment too, while the ground truth includes only the robot's pose. Because of that, the policies, trained on optimal low-dimensional state representations, may achieve performance slightly superior to the ground truth.
} 


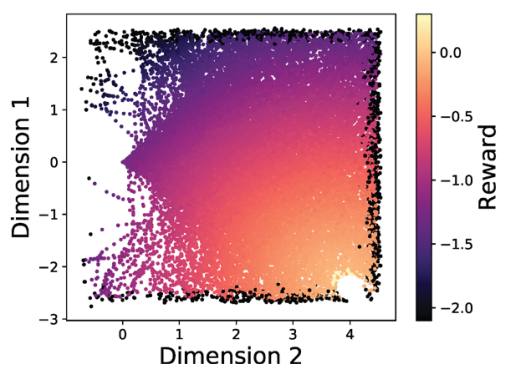

(a) True state values

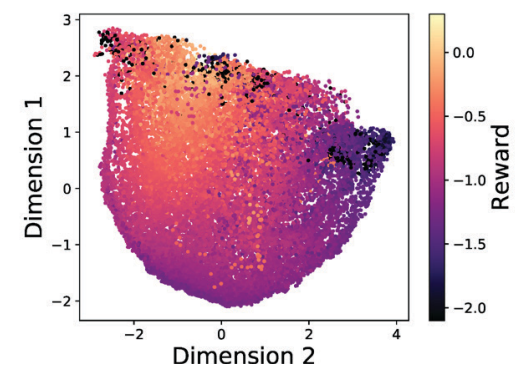

(c) Priors in [12]

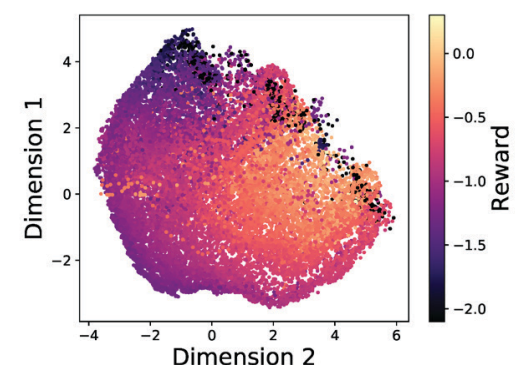

(e) Adaptation of the priors in [83]

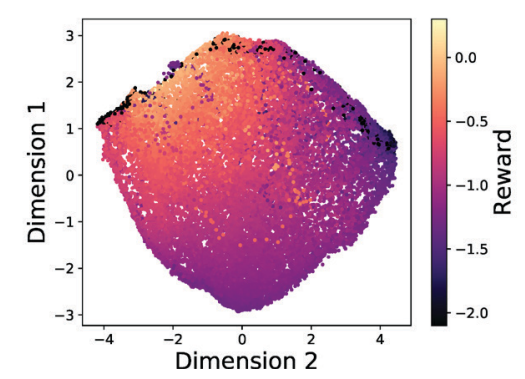

(b) Our Priors

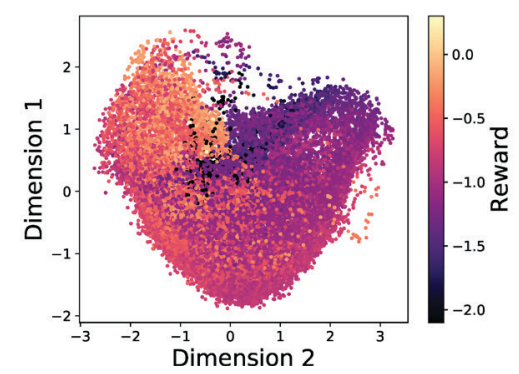

(d) Adaptation of the priors in [63]

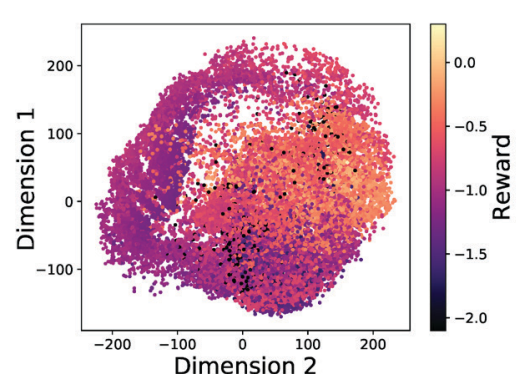

(f) Auto-Encoder

Figure 5.5: True state values (Figure 5.5a), and first two principal components (Figure 5.5b-5.5f), obtained with PCA, of learned state representations for environment Env-1 and target location in the bottom left corner (see Figure 5.4a).

against visual and depth distractors, such as lighting changes and a suddenly appearing moving object on the robot's path to the target. The complete video of our real-world experiments can be found at: https://youtu.be/xujdA4b8-tY. 


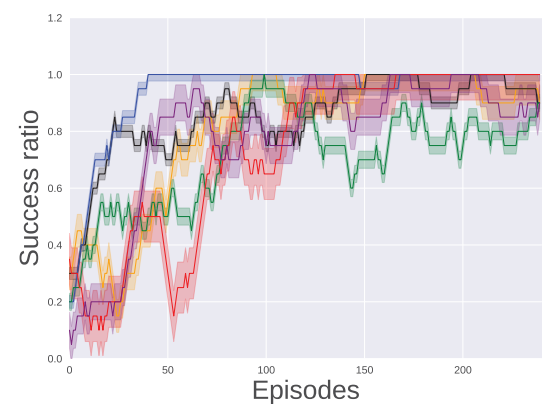

(a) Env-1

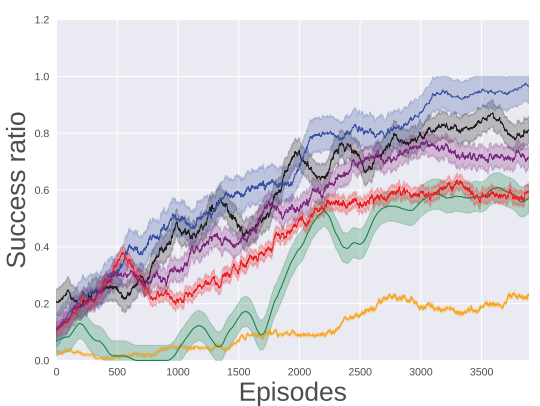

(c) Env-3

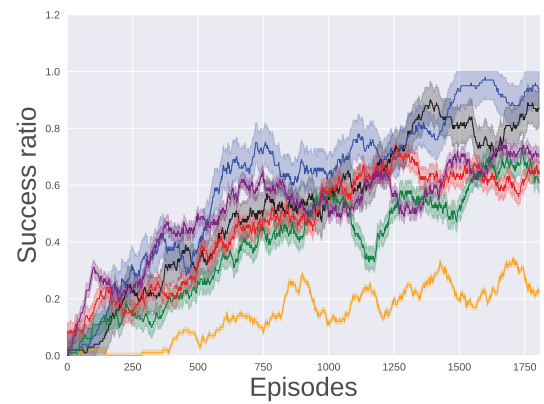

(b) Env-2

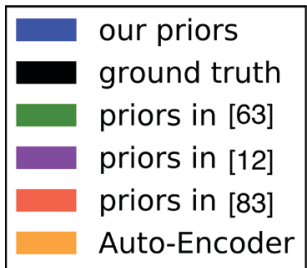

Figure 5.6: Evolution of the success ratio over training in the different environments. The solid line represents the mean and the shade area the variance of the success ratio.

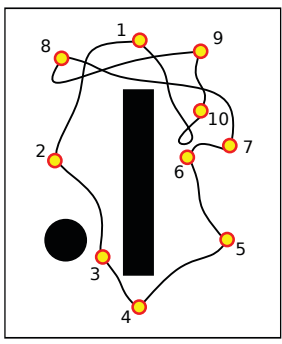

(a) Env-2

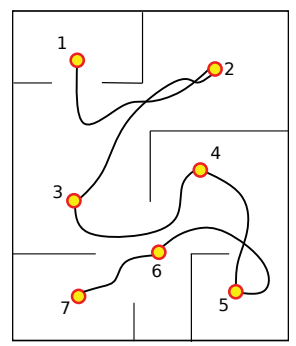

(b) Env-3

Figure 5.7: Trajectory tracking in the virtual test environments. 


\subsection{Conclusions}

In this chapter, we presented an end-to-end Deep Reinforcement Learning framework that explicitly separates the learning of a low-dimensional state representation, given high-dimensional observations, with the policy learning for continuous state and action spaces. We show that we can exploit the underlying continuous action structure by means of the new robotics priors, in Equation (5.1)-(5.4). The new priors allow the learning of a smoother and more coherent representation than the different robotics priors proposed in the literature. This translate into a higher success ratio of the learned policies when trained in different simulation environments. Eventually, the representations and policies learned in the simulation environment can be successfully transferred to the real world without any retraining using real-world data. The compression of high-dimensional observations into a low-dimensional state vector is the key element for transferring the learned models in the simulation environment to the real world. 


\section{Chapter 6}

\section{Low-Dimensional State and Action Representation Learning with MDP Homomorphism Metrics}

Deep Reinforcement Learning has shown its ability in solving complicated problems directly from high-dimensional observations. However, in end-to-end settings, Reinforcement Learning algorithms are not sample efficient and require long training times and quantities of data. In this chapter, we proposed a framework for sample efficient Reinforcement Learning that takes advantage of state and action representations to transform a high-dimensional problem into a low-dimensional one. Moreover, we seek to find the optimal policy mapping latent states to latent actions. Because now the policy is learned on abstract representations, we enforce, using auxiliary loss functions, the lifting of such policy to the original problem domain. Our results show that the novel framework can efficiently learn low-dimensional and interpretable state and action representations and optimal latent policies.

This chapter includes an adaptation of:

Nicolò Botteghi, Mannes Poel, Beril Sirmacek, and Christoph Brune. Low-Dimensional State and Action Representation Learning with MDP Homomorphism Metrics. arXiv, 2021.

Parts of the Section Background were removed as treated in Chapter 2 and 3, and the notation adapted to be consistent with the notation introduced in this thesis. 


\subsection{Introduction}

In the last decade, Deep Reinforcement Learning [1] algorithms have solved increasingly complicated problems in many different domains, spanning from video games [40] to numerous robotics applications [10], in an end-to-end fashion. Despite the success of end-to-end Reinforcement Learning, these methods suffer from low sample efficiency and usually requires lengthy and expensive training procedures to learn optimal behaviours. This problem is even more severe when the true state of the environment is not observable, and the observation space $\mathcal{O}$ or the action space $\mathcal{A}$ are high-dimensional. In end-to-end settings, due to the weak supervision of the reward signal, Reinforcement Learning algorithms are not enforced to learn good state representations of the environment, making the mapping observations to actions challenging to learn and interpret.

State representation learning [6] methods aim at reducing the dimensionality of the observation stream by learning a mapping from the observation space $\mathcal{O}$ to a lower-dimensional state space $\overline{\mathcal{S}}$ containing only the meaningful feature needed for solving a given task. By employing self-supervised auxiliary losses, it is possible to enforce optimal state representation and learn models of the underlying MDP. When policies are learned using the abstract or latent state-space variables, the training time is often reduced, the sample efficiency, the robustness, and generalisation capabilities of the policies grow compared to end-to-end Reinforcement Learning [62], [86] and [87].

While the problem of state representation and observation compression has been extensively treated [6], only a few works have extended the concept of dimensionality reduction to the action space $\mathcal{A}$. In this category, we find the works done in [88], [89] and [90] where low-dimensional action representations are used to improve training efficiency of the agents. In particular, the methods proposed in [89] and [90] learn an action representation using self-supervised approaches.

In this paper, we study the problem of learning state and action representations, in the context of Reinforcement Learning. In particular, with reference to Figure 6.1, we propose a unified framework composed of:

- An encoder neural network $\phi_{e}$ mapping observations to low-dimensional latent states, trained by taking advantage of the MDP homomorphism metrics (see Section 6.2.1). In this way, we can have guarantees on the optimality of the policy learned using the latent state space $\overline{\mathcal{S}}$. 
- The learning of a latent continuous policy $\bar{\pi}$ mapping latent states $\bar{s} \in \overline{\mathcal{S}}$ to latent actions $\bar{a} \in \overline{\mathcal{A}}$.

- The learning of a deterministic action decoder $\delta_{d}$, mapping then continuous latent action space $\overline{\mathcal{A}}$ to the original action space $\mathcal{A}$.

Because the optimal latent policy is learned using state and action representations, it is important to study if such a policy can be lifted to the original problem while preserving its optimality. For this purpose, we employ the notion of MDP homomorphism [91], [92].

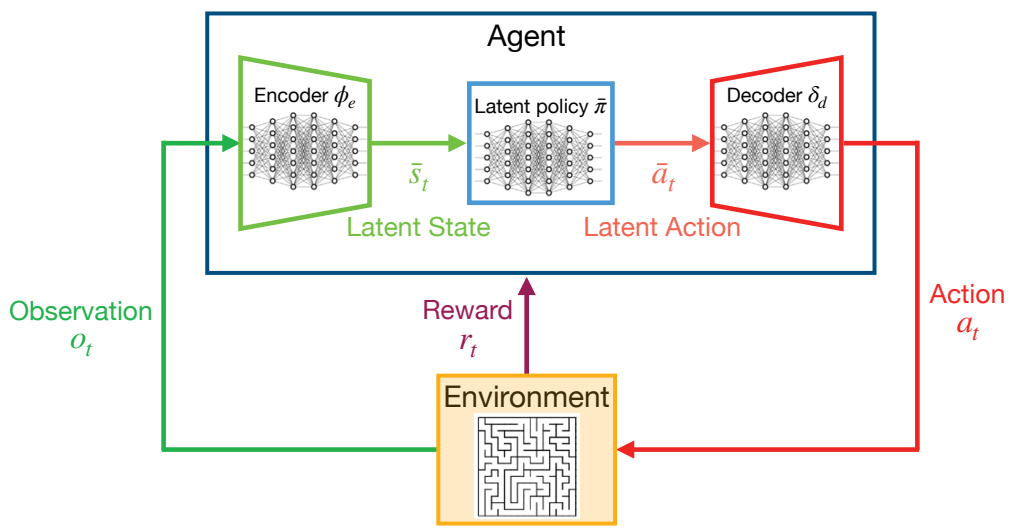

Figure 6.1: Proposed framework combining state and action representation with Reinforcement Learning.

\subsection{Background}

\subsubsection{MDP homomorphism}

When learning representations of the original MDP, we would like to preserve its structure to lift the optimal policies, learned given the representations, to the original MDP by preserving their optimality. This can be done by the notion of MDP homomorphism [91], [92]. 


\section{Definition 6.1: Stochastic MDP Homomorphism}

(Adapted from [92]) A stochastic MDP homomorphism $h$ from an MDP $\mathcal{M}=\langle\mathcal{S}, \mathcal{A}, \mathrm{T}, \mathrm{R}\rangle$ to an $\operatorname{MDP} \overline{\mathcal{M}}=\langle\overline{\mathcal{S}}, \overline{\mathcal{A}}, \overline{\mathrm{T}}, \overline{\mathrm{R}}\rangle$ is a tuple $\left\langle f, g_{s}\right\rangle$, with:

- $f: \mathcal{S} \longrightarrow \overline{\mathcal{S}}$

- $g_{s}: \mathcal{A} \longrightarrow \overline{\mathcal{A}}$

such that the following identities hold:

$$
\begin{gathered}
\forall_{s, s^{\prime} \in \mathcal{S}, a \in \mathcal{A}} \overline{\mathrm{T}}\left(f\left(s^{\prime}\right) \mid f(s), g_{s}(a)\right)=\sum_{s^{\prime \prime} \in\left[s^{\prime}\right]_{f}} \mathrm{~T}\left(s^{\prime \prime} \mid s, a\right) \\
\forall_{s, a \in \mathcal{A}} \quad \overline{\mathrm{R}}\left(f(s), g_{s}(a)\right)=\mathrm{R}(s, a)
\end{gathered}
$$

where $\left[s^{\prime}\right]_{f}$ is the equivalence class of $s^{\prime}$ under Z.

\section{Definition 6.2: Deterministic MDP Homomorphism}

(Adapted from [87]): A deterministic MDP homomorphism $h$ from an MDP $\mathcal{M}=\langle\mathcal{S}, \mathcal{A}, T, R\rangle$ to an $\operatorname{MDP} \overline{\mathcal{M}}=\langle\overline{\mathcal{S}}, \overline{\mathcal{A}}, \overline{\mathrm{T}}, \overline{\mathrm{R}}\rangle$ is a tuple $\left\langle f, g_{s}\right\rangle$, with:

- $f: \mathcal{S} \longrightarrow \overline{\mathcal{S}}$

- $g_{s}: \mathcal{A} \longrightarrow \overline{\mathcal{A}}$

such that the following identities hold:

$$
\begin{gathered}
\forall_{s, s^{\prime} \in \mathcal{S}, a \in \mathcal{A}} \mathrm{T}(s, a)=s^{\prime} \Longrightarrow \overline{\mathrm{T}}\left(f(s), g_{s}(a)\right)=f\left(s^{\prime}\right) \\
\forall_{s \in \mathcal{S}, a \in \mathcal{A}} \quad \overline{\mathrm{R}}\left(f(s), g_{s}(a)\right)=\mathrm{R}(s, a)
\end{gathered}
$$

If Equation (6.1), (6.2) or (6.3), (6.4) are satisfied, the optimal policy $\bar{\pi}$ of the homomorphic image $\overline{\mathcal{M}}$ can be lifted to the original MDP $\mathcal{M}$. Therefore, for deterministic policies $\pi(s)$ and $\bar{\pi}(f(s))$ and a deterministic mapping $f$, we can write:

$$
\pi(s)=\bar{\pi}(f(s))
$$

In this work, we focus on the case of a deterministic $\operatorname{MDP} \mathcal{M}=\langle\mathcal{O}, \mathcal{A}, T, R\rangle$ (all the details in Section 6.4) with deterministic transition function $\mathrm{T}: \mathcal{S} \times \mathcal{A} \longrightarrow \mathcal{S}$, 
state space $\mathcal{S}$ not observable, but Markovian observation space $\mathcal{O}$. With reference to Figure 6.3, we define $f$ as the observation encoder $\phi_{e}: \mathcal{O} \longrightarrow \overline{\mathcal{S}}$ mapping observations to latent states, and $g_{s}$ as the function $\psi_{e}: \overline{\mathcal{S}} \times \mathcal{A} \longrightarrow \overline{\mathcal{A}}$ mapping latent states and actions to latent actions.

\subsection{Related Work}

\subsubsection{Learning State Abstractions}

The notion of MDP homomorphism was first introduced in [91], [92], [93] for exploiting symmetries and similiaties in MDPs. In more recent year, the MDP homomorphism metrics were used for learning low-dimensional state representation in the context of Reinforcement Learning [62], [86], [87]. The MDP homomorphism metrics are used as auxiliary loss functions for training neural networks. When such losses approach zero, it is possible to prove that we have found a homomorphic image of the original MDP. When learning the observation to latent states mapping, a contrastive loss [86], [87] is necessary for preventing the collapse of the mapping. This problem is frequent when the reward function is sparse [62].

Many other state representation learning approaches for Reinforcement Learning have been proposed in literature [6] and most of them employ Auto-Encoder, or $\mathrm{AE}$, reconstruction losses to learn the mapping to the latent state. However, in the context of Reinforcement Learning, where the main goal is only to use the latent state information, the reconstructed observations are usually discarded by making the decoder a non-required and additional complexity. Moreover, AEbased methods tend to struggle to encode and reconstruct non-salient features, and they are easily "distracted" textures or background features. This means that the encoder cannot select between salient and relevant-to-the-task features.

To overcome this problem, several approaches associate to the AE loss, a latent transition loss, or a reward loss, an inverse model loss or a combination of those [78], [94], [95], [96], [97]. In [86], a framework combining model-free and modelbased RL based on the learning of a state representation using multi-objectives loss function is proposed. The collapsing of the state representation due to sparse rewards is tackled by using two contrastive losses.

Eventually, we can find approaches for state representation learning that utilize prior knowledge to shape the latent state space through auxiliary loss functions 
[63], [64], [12]. These methods have proven to be sample efficient and suitable for all the situations in which a low-date regime is required and especially useful in all the cases in which we have prior knowledge of the true environment space, e.g. in robotics where physical laws govern the true state space.

\subsubsection{Learning Action Abstractions}

Action abstraction in MDPs has been first introduced in [67] where a hierarchical decomposition of the policies is proposed to quickly learn skills and complicated tasks by simplifying the policies search space. Here, the low-level policies, i.e. the skill, are executed for a certain amount of steps. Only after their termination, the high-level policies are allowed to act and choose another skill. Several methods have build upon the idea of temporal action abstraction namely the Option framework [98], the Max-Q [99] and the Feudal networks [100].

In [88], the authors utilise prior information over the action space to embed it in a low-dimensional continuous space and allow the generalisation of RL algorithms when the original action space is highly discretised. In [90], the author proposed a method for exploiting the action structure by learning a decoder mapping from chosen low-dimensional continuous action space, in which the policy is learned, to the original action space. This work is the most related to ours. However, we look at the whole problem of state and action representation and their relation.

\subsubsection{Learning State and Action Abstractions}

Our work is related to [101], where state and action embeddings are learned in selfsupervised settings for improving the performance of the Reinforcement Learning agent planning and acting in the learned embedding spaces. Differently, we do not assume full state observability, but we aim at learning a low-dimensional state representation from high-dimensional observations.

\subsection{Methodology}

In this work, we study the interplay between state abstraction and action abstraction. While on one side, state representation learning allows reducing the dimensionality of the input space to exploit similarities and symmetries of the underlying (not-observable) true state space and speed up the learning of the policy 
and value function, we argue that an action representation should do the same. In particular, we aim at exploiting the underlying action space structure by representing it into a low-dimensional continuous space.

With reference to Figure 6.2, we employ a state encoder neural network $\phi_{e}$ : $\mathcal{O} \longrightarrow \overline{\mathcal{S}}$, parametrised by $\boldsymbol{\theta}_{\phi_{e}}$, mapping the observation space ${ }^{1} \mathcal{O}$ to a lowerdimensional latent state space $\overline{\mathcal{S}}$, we learn a continuous latent policy $\bar{\pi}: \overline{\mathcal{S}} \longrightarrow \overline{\mathcal{A}}$, mapping latent states to latent actions, parametrised by a neural network with parameters $\boldsymbol{\theta}_{\bar{\pi}}$, and eventually we map the latent actions back to the original action space $\mathcal{A}$ by means of a decoder $\delta_{d}: \overline{\mathcal{A}} \longrightarrow \mathcal{A}$, parametrised by $\boldsymbol{\theta}_{\delta_{d}}$. Morever, we indicate with $\pi_{i}: \overline{\mathcal{S}} \longrightarrow \mathcal{A}$ the policy mapping latent states to actions ${ }^{2}$, and with $\pi_{o}: \mathcal{S} \longrightarrow \mathcal{A}$ the policy, mapping states to actions, of the original MDP.

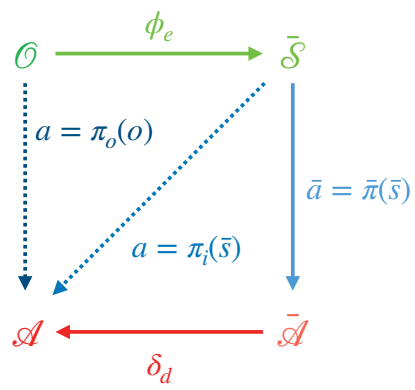

Figure 6.2: Relation between observation, action, latent state, and latent action spaces.

The policy $\bar{\pi}$ is a latent policy, learned based on the latent state space $\overline{\mathcal{S}}$ and latent action space $\overline{\mathcal{A}}$, therefore, to guarantee its optimality and its lifting to the original state space $\mathcal{S}$ and action space $\mathcal{A}$, we use the notion of MDP homomorphism. Herein, we formally study under which conditions an optimal latent policy $\bar{\pi}^{*}$ is equivalent to the optimal intermediate policy $\pi_{i}^{*}$ and to the optimal original policy $\pi_{o}^{*}$.

The proposed framework is self-supervised and does not need labelled data. We only make full use of the experience tuple $\left(o, a, r, o^{\prime}\right)$ collected during the agent's interaction with the environment.

\footnotetext{
${ }^{1}$ We assume that the true state of the environment $\mathcal{S}$ is not directly observable by the agent. However, the agent can perceive the world by means of high-dimensional observations. Similarly to other the work in [87], we restrict to the case of Markovian observations, i.e. a single observation contains enough information for retrieving a good state representation.

${ }^{2}$ We refer to the policy $\pi_{i}$ as the intermediate policy.
} 


\subsubsection{State and Action Representation Learning}

The computational schemes of the proposed framework are presented in Figure 6.3. Our approach combines two elements:

- learning of a low-dimensional state representation $\bar{s}$ using the MDP homomorphism metrics

- learning of a low-dimensional action representation $\bar{a}$ to represent the action space $\mathcal{A}$

such that, as shown in Figure 6.3a, encoding through $\phi_{e}$ the next observation $o^{\prime}$, obtained by applying action $a$ given the observation $o$, is equivalent to the encoding observation $o$ through $\phi_{e}$ and applying the latent action $\bar{a}$.

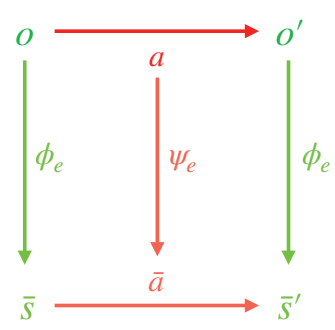

(a)

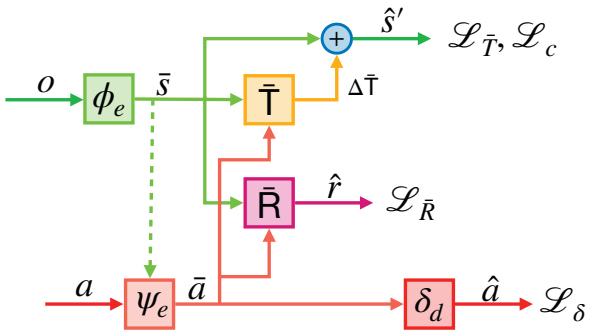

(b)

Figure 6.3: Computational schemes of the proposed framework.

\section{Learning Low-dimensional State Representations}

We aim at learning an MDP homomorphism $\overline{\mathcal{M}}=\langle\overline{\mathcal{S}}, \overline{\mathcal{A}}, \overline{\mathrm{T}}, \overline{\mathrm{R}}\rangle$ of the original $\operatorname{MDP} \mathcal{M}=\langle\mathcal{O}, \mathcal{A}, \mathrm{T}, \mathrm{R}\rangle$. Similarly to [62] and [87], the observation encoder $\phi_{e}$ is trained by means of the MDP homomorphism metrics, in Equation (6.3) and (6.4), and without the need of an observation decoder.

With reference to Figure $6.3 \mathrm{~b}$, we define a latent transition model $\overline{\mathrm{T}}: \overline{\mathcal{S}} \times \overline{\mathcal{A}} \longrightarrow$ $\overline{\mathcal{S}}$, predicting the next latent state given a latent state-action pair, and a latent reward model $\overline{\mathrm{R}}: \overline{\mathcal{S}} \times \overline{\mathcal{A}} \longrightarrow \mathbb{R}$, predicting the reward of a latent state-action pair. Both mapping are learned with neural networks with parameters' vector $\boldsymbol{\theta}_{\overline{\mathrm{T}}}$ and $\boldsymbol{\theta}_{\overline{\mathrm{R}}}$ respectively. 
Firstly, the transition loss, in Equation (6.6), is used to enforce that transitions $\mathrm{T}(o, a)$ in original MDP $\mathcal{M}$ correspond to transitions $\overline{\mathrm{T}}(\bar{s}, \bar{a})$ in the latent $\mathrm{MDP}$ $\overline{\mathcal{M}}$. Similarly to [87], [102], we model the transitions in the latent spaces as $\overline{\mathrm{T}}(\bar{s}, \bar{a})=\Delta \overline{\mathrm{T}}(\bar{s}, \bar{a})+\bar{s}$.

$$
\begin{aligned}
\mathcal{L}_{\overline{\mathrm{T}}}\left(\boldsymbol{\theta}_{\phi_{e}}, \boldsymbol{\theta}_{\overline{\mathrm{T}}}, \boldsymbol{\theta}_{\psi_{e}}\right) & =\mathbb{E}\left[\left\|\bar{s}^{\prime}-\hat{s}^{\prime}\right\|_{2}\right] \\
& =\mathbb{E}\left[\left\|\bar{s}^{\prime}-\overline{\mathrm{T}}\left(\bar{s}, \bar{a} ; \boldsymbol{\theta}_{\overline{\mathrm{T}}}\right)\right\|_{2}\right] \\
& =\mathbb{E}\left[\left\|\bar{s}^{\prime}-\left(\Delta \overline{\mathrm{T}}\left(\bar{s}, \bar{a} ; \boldsymbol{\theta}_{\overline{\mathrm{T}}}\right)+\bar{s}\right)\right\|_{2}\right] \\
& =\mathbb{E}\left[\left\|\phi_{e}\left(o^{\prime} ; \boldsymbol{\theta}_{\phi_{e}}\right)-\left(\Delta \overline{\mathrm{T}}\left(\phi_{e}\left(o ; \boldsymbol{\theta}_{\phi_{e}}\right), \bar{a} ; \boldsymbol{\theta}_{\overline{\mathrm{T}}}\right)+\phi_{e}\left(o ; \boldsymbol{\theta}_{\phi_{e}}\right)\right)\right\|_{2}\right] \\
& =\mathbb{E}\left[\| \phi_{e}\left(o^{\prime} ; \boldsymbol{\theta}_{\phi_{e}}\right)-\left(\Delta \overline{\mathrm{T}}\left(\phi_{e}\left(o ; \boldsymbol{\theta}_{\phi_{e}}\right), \psi_{e}\left(\bar{s}, a ; \boldsymbol{\theta}_{\psi_{e}}\right) ; \boldsymbol{\theta}_{\overline{\mathrm{T}}}\right)\right.\right. \\
& \left.\left.+\phi_{e}\left(o ; \boldsymbol{\theta}_{\phi_{e}}\right)\right) \|_{2}\right]
\end{aligned}
$$

where the target next latent state $\bar{s}^{\prime}=\phi_{e}\left(o^{\prime} ; \boldsymbol{\theta}_{\phi_{e}}\right)$ is generated by encoding the next observation $o^{\prime}$, while the next latent state prediction $\hat{s}^{\prime}=\overline{\mathrm{T}}\left(\bar{s}, \bar{a} ; \boldsymbol{\theta}_{\overline{\mathrm{T}}}\right)$ is generated from the encoding $\bar{s}=\phi_{e}\left(o ; \boldsymbol{\theta}_{\phi_{e}}\right)$ of the observation $o$, the action $a$, and the latent transition model $\overline{\mathrm{T}}\left(\bar{s}, \bar{a} ; \boldsymbol{\theta}_{\overline{\mathrm{T}}}\right)$.

Secondly the reward loss, in Equation (6.7), is used to enforce the same reward function in the original $\operatorname{MDP} \mathcal{M}$ and the latent $\operatorname{MDP} \overline{\mathcal{M}}$.

$$
\begin{aligned}
\mathcal{L}_{\overline{\mathrm{R}}}\left(\boldsymbol{\theta}_{\phi_{e}}, \boldsymbol{\theta}_{\overline{\mathrm{R}}}, \boldsymbol{\theta}_{\psi_{e}}\right) & =\mathbb{E}\left[\|r-\hat{r}\|_{2}\right] \\
& =\mathbb{E}\left[\left\|r-\overline{\mathrm{R}}\left(\bar{s}, \bar{a} ; \boldsymbol{\theta}_{\overline{\mathrm{R}}}\right)\right\|_{2}\right] \\
& =\mathbb{E}\left[\left\|r-\overline{\mathrm{R}}\left(\phi_{e}\left(o ; \boldsymbol{\theta}_{\phi_{e}}\right), \bar{a} ; \boldsymbol{\theta}_{\overline{\mathrm{R}}}\right)\right\|_{2}\right] \\
& =\mathbb{E}\left[\left\|r-\overline{\mathrm{R}}\left(\phi_{e}\left(o ; \boldsymbol{\theta}_{\phi_{e}}\right), \psi_{e}\left(\bar{s}, a ; \boldsymbol{\theta}_{\psi_{e}}\right) ; \boldsymbol{\theta}_{\overline{\mathrm{R}}}\right)\right\|_{2}\right]
\end{aligned}
$$

where $r$ is the reward obtained by interacting with the environment and $\hat{r}=$ $\overline{\mathrm{R}}\left(\bar{s}, \bar{a} ; \boldsymbol{\theta}_{\overline{\mathrm{R}}}\right)$ is the predicted reward using the learned reward model $\overline{\mathrm{R}}\left(\bar{s}, \bar{a} ; \boldsymbol{\theta}_{\overline{\mathrm{R}}}\right)$, the current observation $o$, and the action $a$.

Additionally, we used the hinge loss in Equation (6.8) to prevent the trivial embedding in which all the latent states are mapped to the zero vector ${ }^{3}$ as this

\footnotetext{
${ }^{3}$ This is often the case when the rewards are sparse [87], [102].
} 
would not be an MDP homomorphism.

$$
\begin{aligned}
\mathcal{L}_{c}\left(\boldsymbol{\theta}_{\phi_{e}}, \boldsymbol{\theta}_{\overline{\mathrm{T}}}, \boldsymbol{\theta}_{\psi_{e}}\right) & =\mathbb{E}\left[\max \left(0, \epsilon-\left\|\bar{s}_{n}^{\prime}-\hat{s}^{\prime}\right\|_{2}\right]\right) \\
& =\mathbb{E}\left[\max \left(0, \epsilon-\left\|\bar{s}_{n}^{\prime}-\overline{\mathrm{T}}\left(\bar{s}, \bar{a} ; \boldsymbol{\theta}_{\overline{\mathrm{T}}}\right)\right\|_{2}\right]\right) \\
& =\mathbb{E}\left[\operatorname { m a x } \left(0, \epsilon-\| \phi_{e}\left(o_{n}^{\prime} ; \boldsymbol{\theta}_{\phi_{e}}\right)-\left(\Delta \overline{\mathrm{T}}\left(\phi_{e}\left(o ; \boldsymbol{\theta}_{\phi_{e}}\right), \bar{a} ; \boldsymbol{\theta}_{\overline{\mathrm{T}}}\right)+\right.\right.\right. \\
& \left.\left.\left.+\phi_{e}\left(o ; \boldsymbol{\theta}_{\phi_{e}}\right)\right) \|_{2}\right)\right] \\
& =\mathbb{E}\left[\operatorname { m a x } \left(0, \epsilon-\| \phi_{e}\left(o_{n}^{\prime} ; \boldsymbol{\theta}_{\phi_{e}}\right)-\left(\Delta \overline{\mathrm{T}}\left(\phi_{e}\left(o ; \boldsymbol{\theta}_{\phi_{e}}\right), \psi_{e}\left(\bar{s}, a ; \boldsymbol{\theta}_{\psi_{e}}\right) ; \boldsymbol{\theta}_{\overline{\mathrm{T}}}\right)+\right.\right.\right. \\
& \left.\left.\left.+\phi_{e}\left(o ; \boldsymbol{\theta}_{\phi_{e}}\right)\right) \|_{2}\right)\right]
\end{aligned}
$$

where $\epsilon$ is the hinge parameter governing the effect of the negative distance, and $o_{n}^{\prime}$ is a randomly sampled observation, not a successor of the observation $o$.

The total loss for enforcing the MDP homomorphism is shown in Equation $(6.9)$.

$$
\mathcal{L}_{\mathrm{MDP}}=\omega_{\overline{\mathrm{T}}} \mathcal{L}_{\overline{\mathrm{T}}}\left(\boldsymbol{\theta}_{\phi_{e}}, \boldsymbol{\theta}_{\overline{\mathrm{T}}}, \boldsymbol{\theta}_{\psi_{e}}\right)+\omega_{\overline{\mathrm{R}}} \mathcal{L}_{\overline{\mathrm{R}}}\left(\boldsymbol{\theta}_{\phi_{e}}, \boldsymbol{\theta}_{\overline{\mathrm{R}}}, \boldsymbol{\theta}_{\psi_{e}}\right)+\omega_{c} \mathcal{L}_{c}\left(\boldsymbol{\theta}_{\phi_{e}}, \boldsymbol{\theta}_{\overline{\mathrm{T}}}, \boldsymbol{\theta}_{\psi_{e}}\right)
$$

where $\omega_{\overline{\mathrm{T}}}, \omega_{\overline{\mathrm{R}}}$, and $\omega_{c}$ are three constants weighting the contribution of the each individual loss function.

\section{Learning Low-dimensional Action Representations}

Our second objective is to exploit similarities and structure of the action space $\mathcal{A}$. To do that we employ an action encoder $\psi_{e}: \overline{\mathcal{S}} \times \mathcal{A} \longrightarrow \overline{\mathcal{A}}$, mapping latent states and actions to state-dependent latent actions ${ }^{4}$, and an action decoder $\delta_{d}: \overline{\mathcal{A}} \longrightarrow \mathcal{A}$ mapping latent actions to the original action space (see Figure 6.3b).

In our work, we study the case of a discrete action space $\mathcal{A}$, therefore, to train latent model $\psi_{e}$ and decoder $\delta_{d}$, it is possible to use the cross-entropy $\operatorname{loss}^{5}$ in

\footnotetext{
${ }^{4}$ The action encoder can be solely chosen a function of the actions $\psi_{e}: \mathcal{A} \longrightarrow \overline{\mathcal{A}}$.

${ }^{5}$ In continuous action spaces, it is possible to use simply the mean squared error loss between the action and the predicted action using the models.
} 
Equation (6.10).

$$
\begin{aligned}
\mathcal{L}_{\delta}\left(\boldsymbol{\theta}_{\psi_{e}}, \boldsymbol{\theta}_{\delta_{d}}, \boldsymbol{\theta}_{\psi_{e}}\right) & =\mathbb{E}\left[-\sum_{i=0}^{K}\left(a_{i} \log \left(\hat{a}_{i}\right)\right)\right] \\
& =\mathbb{E}\left[-\sum_{i=0}^{K}\left(a_{i} \log \left(\delta_{d}\left(\bar{a} ; \boldsymbol{\theta}_{\delta_{d}}\right)_{i}\right)\right]\right. \\
& =\mathbb{E}\left[-\sum_{i=0}^{K}\left(a_{i} \log \left(\delta_{d}\left(\psi_{e}\left(\bar{s}, a ; \boldsymbol{\theta}_{\psi_{e}}\right)\right) ; \boldsymbol{\theta}_{\delta_{d}}\right)_{i}\right)\right]
\end{aligned}
$$

where $a_{i}$ is the $i$-th component of one-hot encoded action $a$ and $\hat{a}_{i}$ is the $i$-th component of the normalised logit corresponding to the predicted action $\hat{a}$. A similar loss function is employed in [90] and [101].

\section{The Complete Loss Function}

The total loss function that is minimised for training our neural network models is shown in Equation (6.11) and it is equal to the weighted sum of the four different losses shown in Equation (6.6)-(6.8), and (6.10).

$$
\begin{gathered}
\min _{\boldsymbol{\theta}_{\phi_{e}}, \boldsymbol{\theta}_{\psi_{e}}, \boldsymbol{\theta}_{\delta_{d}}, \boldsymbol{\theta}_{\overline{\mathrm{T}}}, \boldsymbol{\theta}_{\overline{\mathrm{R}}}} \quad \omega_{\overline{\mathrm{T}}} \mathcal{L}_{\overline{\mathrm{T}}}\left(\boldsymbol{\theta}_{\phi_{e}}, \boldsymbol{\theta}_{\overline{\mathrm{T}}}, \boldsymbol{\theta}_{\psi_{e}}\right)+\omega_{\overline{\mathrm{R}}} \mathcal{L}_{\overline{\mathrm{R}}}\left(\boldsymbol{\theta}_{\phi_{e}}, \boldsymbol{\theta}_{\overline{\mathrm{R}}}, \boldsymbol{\theta}_{\psi_{e}}\right)+\omega_{c} \mathcal{L}_{c}\left(\boldsymbol{\theta}_{\phi_{e}}, \boldsymbol{\theta}_{\overline{\mathrm{T}}}, \boldsymbol{\theta}_{\psi_{e}}\right)+ \\
+\omega_{\delta_{d}} \mathcal{L}_{\delta}\left(\boldsymbol{\theta}_{\psi_{e}}, \boldsymbol{\theta}_{\delta_{d}}\right)
\end{gathered}
$$

It is worth mentioning that the action encoder $\psi_{e}$ is affected by the MDP homomorphism losses, in Equation (6.9), and the action decoder loss, in Equation (6.10). In this way, we aim at learning an action representation that a) exploits symmetries and b) allows reconstruction of the true action space.

\subsubsection{Optimality of the Policies}

In this section, we first study the relation between the latent policy $\bar{\pi}: \overline{\mathcal{S}} \longrightarrow \overline{\mathcal{A}}$ and the policy $\pi_{o}: \mathcal{O} \longrightarrow \mathcal{A}$. Then, we study the relation between the policy $\bar{\pi}$ and the intermediate policy $\pi_{i}: \overline{\mathcal{S}} \longrightarrow \mathcal{A}$. Eventually, we study the relation between $\pi_{o}$ and $\pi_{i}$. 


\section{Proposition 6.1: Equivalence of $\pi^{*}$ and $\pi_{0}^{*}$}

Accordingly to [62], [67], [87], [93] when the loss in Equation (6.9) approaches zero, the $\operatorname{MDP} \overline{\mathcal{M}}=\langle\overline{\mathcal{S}}, \overline{\mathcal{A}}, \overline{\mathrm{T}}, \overline{\mathrm{R}}\rangle$ is an (approximate) homomorphism of the original $\operatorname{MDP} \mathcal{M}=\langle\mathcal{O}, \mathcal{A}, \mathrm{T}, \mathrm{R}\rangle$. The optimal latent policy $\bar{\pi}^{*}: \overline{\mathcal{S}} \longrightarrow \mathcal{A}$ can be lifted to the original MDP by preserving its optimality. Therefore, with reference to Figure 6.2, the optimal latent policy $\bar{\pi}^{*}: \overline{\mathcal{S}} \longrightarrow \overline{\mathcal{A}}$ is equivalent to the optimal policy $\pi_{o}^{*}: \mathcal{O} \longrightarrow \mathcal{A}$.

\section{Proposition 6.2: Equivalence of $\bar{\pi}^{*}$ and $\pi_{i}^{*}$}

For all deterministic functions $\delta_{d}$, the gradient $\nabla_{\boldsymbol{\theta}_{\bar{\pi}}} J_{\bar{\pi}}\left(\boldsymbol{\theta}_{\bar{\pi}}\right)$ of the performance measure of the latent policy $\bar{\pi}: \overline{\mathcal{S}} \longrightarrow \overline{\mathcal{A}}$ is equivalent to the gradient $\nabla_{\boldsymbol{\theta}_{\bar{\pi}}} J_{\pi_{i}}\left(\boldsymbol{\theta}_{\bar{\pi}}, \boldsymbol{\theta}_{\delta_{d}}\right)$ of the performance measure the intermediate policy $\pi_{i}: \overline{\mathcal{S}} \longrightarrow \mathcal{A}$ :

$$
\nabla_{\boldsymbol{\theta}_{\bar{\pi}}} J_{\pi_{i}}\left(\boldsymbol{\theta}_{\bar{\pi}}, \boldsymbol{\theta}_{\delta_{d}}\right)=\nabla_{\boldsymbol{\theta}_{\bar{\pi}}} J_{\bar{\pi}}\left(\boldsymbol{\theta}_{\bar{\pi}}\right)
$$

Therefore ascending the gradient of $\bar{\pi}$ is equivalent to ascending the gradient of $\pi_{i}$. The complete proof is shown in Appendix A.

\section{Proposition 6.3: Equivalence of $\pi_{i}^{*}$ and $\pi_{0}^{*}$}

As consequence of Proposition 6.1 and Proposition 6.2, an optimal internal policy $\pi_{i}^{*}: \overline{\mathcal{S}} \longrightarrow \mathcal{A}$ is equivalent to an optimal policy $\pi_{o}^{*}: \mathcal{O} \longrightarrow \mathcal{A}$. Thus, an optimal latent policy $\bar{\pi}^{*}$ for the $\operatorname{MDP} \overline{\mathcal{M}}$ is equivalent to an optimal intermediate policy $\pi_{i}^{*}$ and to an optimal policy $\pi_{o}^{*}$ for the original MDP $\mathcal{M}$.

\subsubsection{Neural Network Architectures}

For learning the latent policy $\bar{\pi}$, any Reinforcement Learning algorithm that can deal with continuous state and action space can be used. Here, we use TD3 (see Section 3.2.3) with the implementation provided in [44]. Actor and critic networks are composed of two fully-connected layers with 256 units with ReLU activation. The output layer of the actor has tanh activation and outputs latent actions, while 
the critics have linear activation and output the Q-values of the state-action pairs. The actor and a critic neural networks are shown in Figure 6.4.

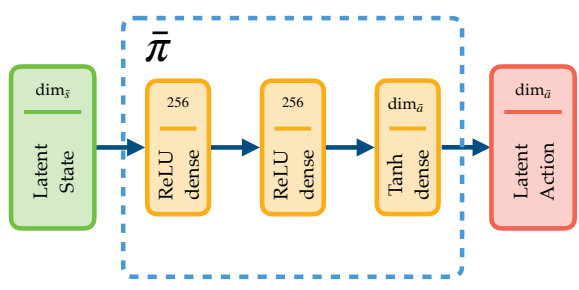

(a) Actor Model

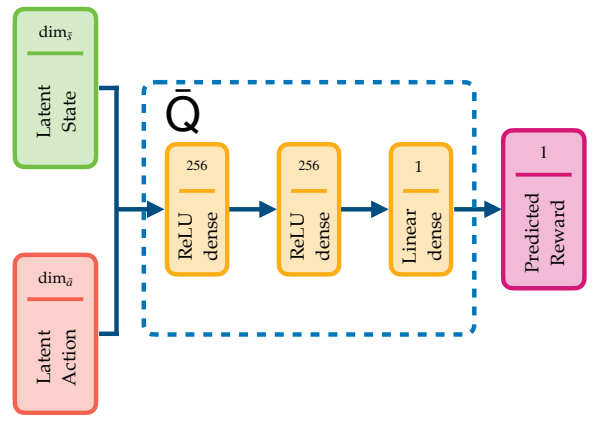

(b) Critic Model

Figure 6.4: Neural network architecture of the latent policy $\bar{\pi}$ and the latent action-value function $\bar{Q}$

The encoder $\phi_{e}$ is composed of two convolutional layers, with 32 and 64 filters of size $3 \times 3$ and $5 \times 5$ respectively, with ReLU activations, two fully-connected layers, with 64 and 32 units, with ReLU activation, and a final fully-connected layer with linear activation outputting latent states. The complete architecture can be seen in Figure 6.5.

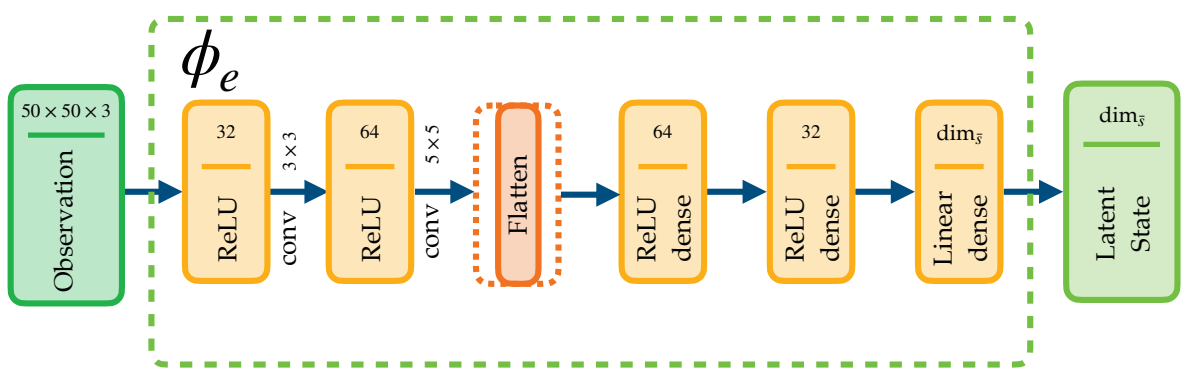

Figure 6.5: Neural network architecture of the observation encoder $\phi_{e}$

The transition model and the reward model share a similar architecture with two fully-connected layers, with 64 and 32 units, and ReLU activation respectively, and an output layer with linear activation, as shown in Figure 6.6. Similar architectures are employed in [87]. 


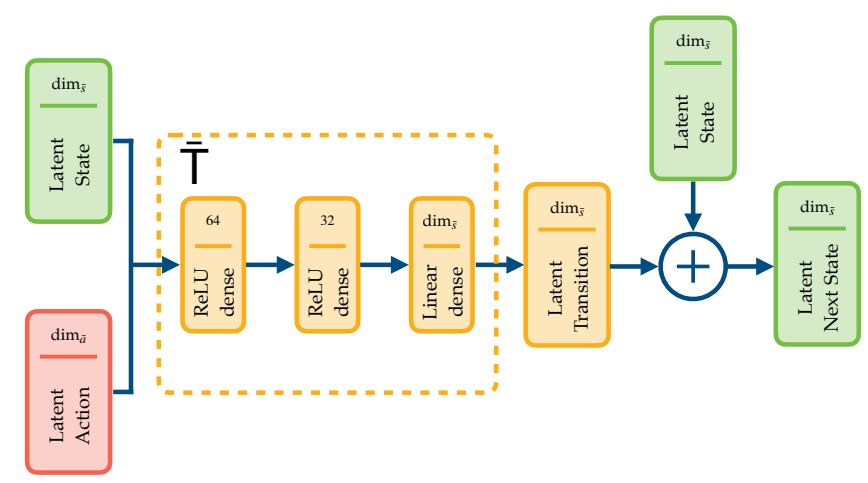

(a) Transition Model

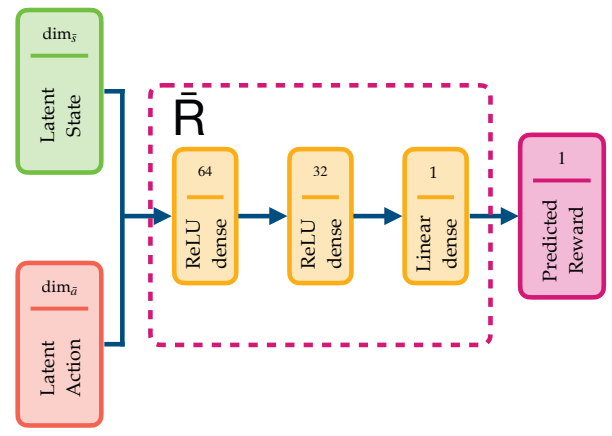

(b) Reward Model

Figure 6.6: Neural network architecture of the transition model $\overline{\mathrm{T}}$ and reward model $\overline{\mathrm{R}}$.

Eventually, the action encoder comprises two fully-connected layers, with 64 and 32 units and ReLU activation. The output layer has tanh activation to bound the latent action space in $[-1,1]$. The action decoder has a similar architecture except a softmax output activation to map latent actions to one-hot encoded action of the original action space. A similar architecture is employed in [90].

\subsection{Experimental Design}

\subsubsection{Grid-World}

The grid-world can be seen as the simple mobile robot navigation problem, where the agent has to navigate the robot to a target cell of the grid, and it is enforced to 

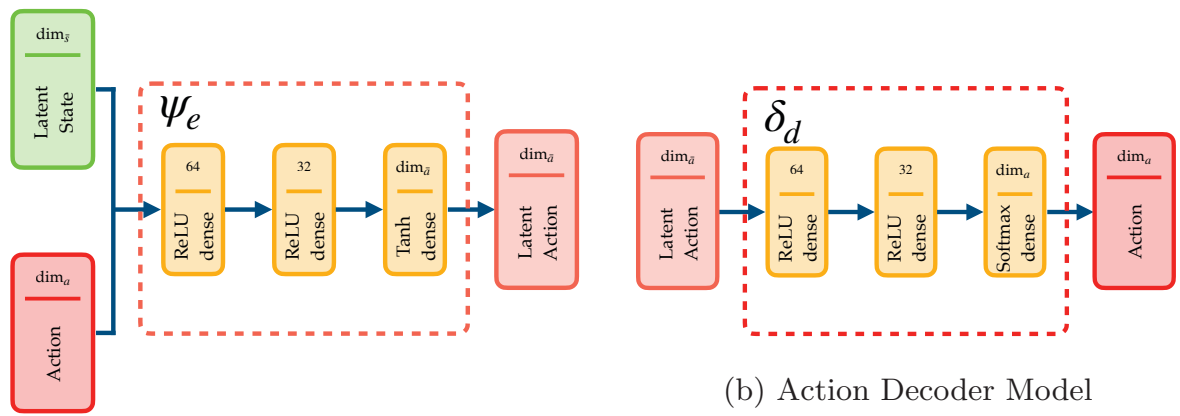

(b) Action Decoder Model

(a) Action Encoder Model

Figure 6.7: Neural network architecture of the action encoder model $\psi_{e}$ and the action decoder model $\delta_{d}$.

move along the underlying grid. At each training episode, the robot is randomly spawned in different positions of the maze. Grid-worlds are used in [87], [102], [90], and [101]. In our experiments, the agent can observe the maze through RGB images of size $50 \times 50$. Examples of mazes and agent's observations can be found in Figure 6.8. For the grid-world experiments, we adapted the environments in [103] and [102].

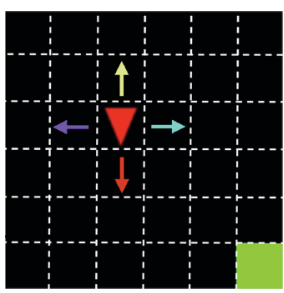

(a) $6 \times 6$ maze -1 object

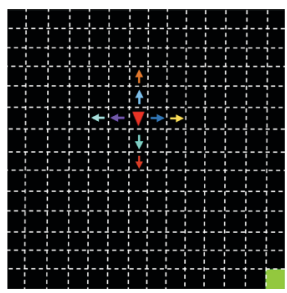

(b) $14 \times 14$ maze -1 object

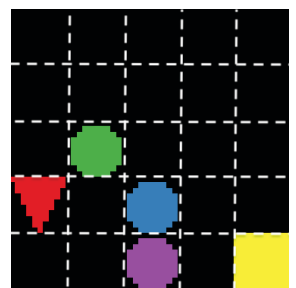

(c) $5 \times 5$ maze -1 objects, 3 distractors

Figure 6.8: Observations from different grid-world environments. The triangles indicate the robots controlled by the agent, the squares the target positions, the circles are visual distractors, and the arrows represent the different actions of the agent.

We use for all the experiments a distance-based reward function, as shown in Equation (6.13). 


$$
\mathrm{R}(s, a)= \begin{cases}r_{\text {reached }}, & s=s_{\text {goal }} \\ -\eta d, & \text { otherwise }\end{cases}
$$

where $r_{\text {reached }}$ is a bonus for reaching the goal position, $d$ is the Manhattan distance robot-goal normalised over the number of cells of the maze, and $\eta$ is a scaling factor. Distance-based reward functions are a natural choice for robot navigation tasks.

We experiment in:

- $6 \times 6$ maze in which the agent has to steer a single robot (red triangle in Figure 6.8a) to a target position (green square) by choosing among four possible actions.

- $14 \times 14$ maze in which the agent has to steer a single robot (red triangle in Figure $6.8 \mathrm{~b}$ ) to a target position (green square) by choosing among eight possible actions.

- $5 \times 5$ maze in which the agent has to steer a single robot (red triangle in Figure 6.8c) to a target position (yellow square) by choosing among four possible actions per object. During the training of the policy, up to three unseen distractors (circles) randomly move across the maze.

\subsubsection{Mobile Robot Navigation}

Secondly, we test our approach on a simple mobile robot navigation task with continuous underlying state space. The mobile robot (Pioneer p3dx) is simulated on VRep [104] using the PyRep interface [105]. The agent receives $48 \times 48$ RGB images coming from an onboard camera, and its action space is composed of three and eight different discrete actions.

The reward function is similar to the one used in the grid-world but with a penalty for colliding with the walls. The complete reward function is shown in Equation (6.14).

$$
\mathrm{R}(s, a)= \begin{cases}r_{\text {reached }}, & d \leq d_{\text {min }} \\ r_{\text {crashed }}, & s=s_{t s} \\ -\eta d, & \text { otherwise }\end{cases}
$$




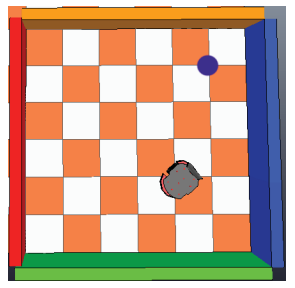

(a) Robot navigation task

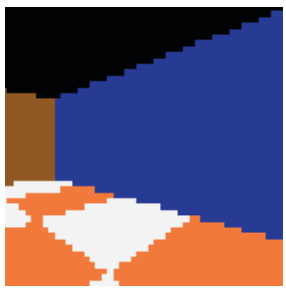

(b) Example of observation

Figure 6.9: The agent needs to steer the robot to the target (purple circle in Figure $6.9 \mathrm{a}$ ) by relying on RGB images coming from an onboard camera (Figure 6.9b).

where $r_{\text {reached }}$ is a bonus for reaching the goal position, $r_{\text {crashed }}$ is a penalty for colliding with an obstacle, i.e. reaching a terminal state $s_{t s}, d$ is the Euclidean distance robot-goal, and $\eta$ is a scaling factor.

\subsubsection{Comparison of the Learned State Representations}

To assess the validity of our approach, we qualitatively compare the learned state representation of our method with:

- MDP-H: adaptation of the plannable MDP homomorphism framework proposed in [87] in which we learn the state representation by means of the auxiliary losses in Equation (6.6), (6.7) and (6.8), but without the action representation module $\psi_{e}$ and only using the true action $a$.

- D-MDP: adaptation of the Deep MDP framework proposed in [62] in which we learn the state representation by means of the auxiliary losses in Equation (6.6) and (6.7), but without the action representation module $\psi_{e}$ and only using the true action $a$.

- JSAE: adaptation of the joint state-action embeddings framework proposed in [101].

- JSAE-C: Adaptation of the joint state-action embeddings framework proposed in [101] with the addition of the contrastive loss, in Equation (6.8), for preventing the trivial embedding in which all states are mapped to the zero vector. 
For the fairness of comparison, we train all the neural networks using the same data-set of samples collected through random interaction with the environments, the same network architectures for state encoder $\phi_{e}$, latent transition model $\overline{\mathrm{T}}$, and reward model $\overline{\mathrm{R}}$, same learning rate, batch size, latent state space dimensionality, and three random seeds.

The list of hyperparameters used is shown in Table 6.1.

\begin{tabular}{||c|c||}
\hline Hyperparameter & Value \\
\hline \hline Latent state dimension $\left(\operatorname{dim}_{\bar{s}}\right)$ & 10 \\
Latent transition dimension $\left(\operatorname{dim}_{\bar{s}}\right)$ & 10 \\
Latent action dimension $\left(\operatorname{dim}_{\bar{a}}\right)$ & 5 \\
Learning rate & 0.0005 \\
Batch size & 256 \\
Training Epochs & 100 \\
optimiser & ADAM \\
\hline
\end{tabular}

Table 6.1: Hyperparameters of the experiments

\subsubsection{Comparison of the Learned Policies}

After learning the state (and action) representation, we aim at learning the optimal policy given such a representation. We, therefore, compare, in terms of the average number of steps the agents take over training, the performance of our approach, learning a continuous latent policy given a fixed state and action representation, with the performance of a Deep Q-Network agent [41], or DQN, mapping latent states directly to actions. In the latter, the state representation is learned with the method proposed in [87], but without the discretisation step employed by the authors. The discretisation of the latent state space would limit the applicability only to MDPs with underlying discrete state space. While this is true for the grid-worlds in Figure 6.8, in the case of mobile robot navigation, in Figure 6.9, the underlying state space is continuous.

Similarly to [106], we are interested in the best-performing agents; therefore, we train each policy (TD3 and DQN) using ten different seeds, but we plot the mean and the variance of the best three seeds per algorithm.

The list of hyperparameters used is shown in Table 6.2. 


\begin{tabular}{||c|c||}
\hline Hyperparameter & Value \\
\hline \hline Latent state dimension $\left(\operatorname{dim}_{\bar{s}}\right)$ & 10 \\
Latent action dimension $\left(\operatorname{dim}_{\bar{a}}\right) \mathrm{TD} 3$ & 5 \\
Action dimension DQN & $3,4,8$ \\
Learning rate DQN & 0.0005 \\
Learning rate Actor & 0.0005 \\
Learning rate Critic & 0.0005 \\
Batch size & 64 \\
optimiser & ADAM \\
$\epsilon$-greedy coefficient & 0.25 \\
Random noise $\sigma$ & 0.35 \\
\hline \multicolumn{2}{|c}{}
\end{tabular}

Table 6.2: Hyperparameters of the experiments

\subsection{Results}

\subsubsection{Grid-World}

\section{Comparison of the Learned Representations}

We first analyse the learned state representations obtained with the different approaches discussed in Section 6.5 .3 by plotting the state predictions generated by encoding a set of randomly collected observations of the different mazes. The state representation learned in the $14 \times 14$ grid-world are shown in Figure 6.10. Additionally, the learned state representation in the $6 \times 6$ grid-world are shown in Appendix B (Figure B.2).

Only our approach and the plannable MDP homomorphism framework [87] can retrieve the underlying grid structure of the true state space. The Deep MDP [62] can still retrieve a partial structure, while the JSAE struggles even with the addition of the contrastive loss.

In Figure 6.11, we also show the learned action representations (Figure 6.11b) and learned transitions $\Delta \overline{\mathrm{T}}$ (Figure $6.11 \mathrm{~d}$ ) in the $14 \times 14$ mazes in Figure $6.8^{6}$. The latent transitions resemble the true transitions of the agent in the grid-world.

\section{Comparison of the Learned Policies}

In Figure 6.12, the performance of the two agents is compared for the different gridworlds. As soon as the state and action space grows, e.g. maze $14 x 14$ with eight

\footnotetext{
${ }^{6}$ Again the results in the $6 \times 6$ grid-world are shown in Appendix B (Figure B.1).
} 


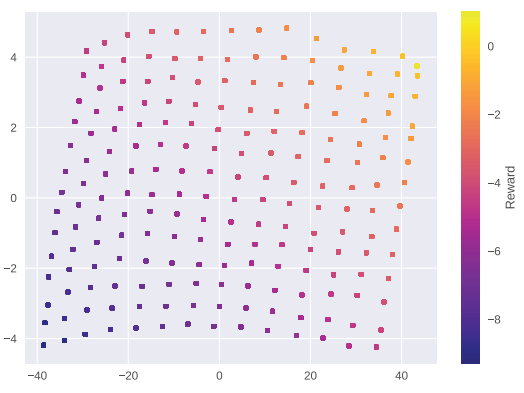

(a) Our

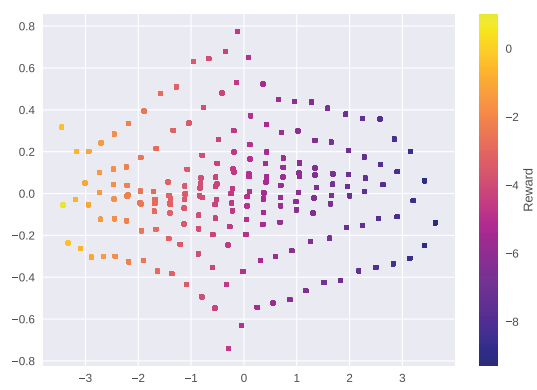

(c) D-MDP [62]

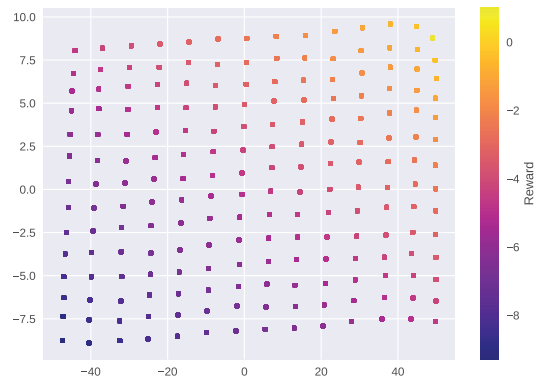

(b) MDP-H $[87]$

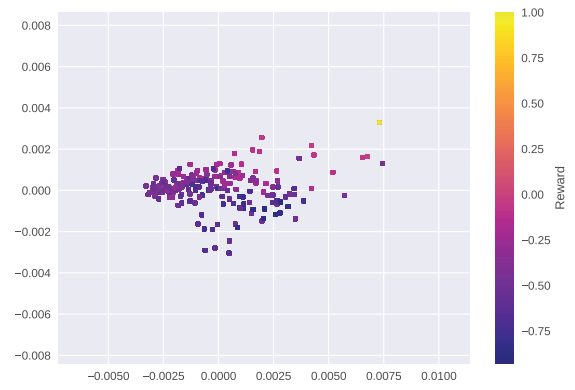

(d) JSAE [101]

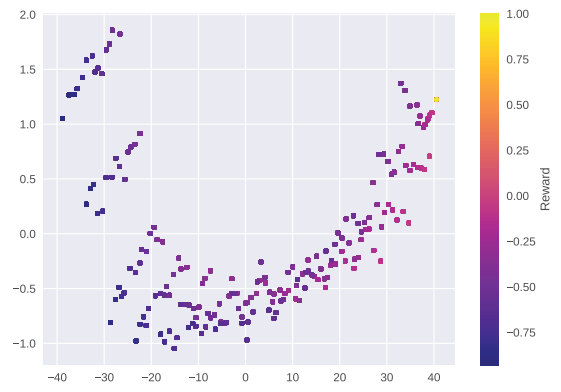

(e) JSAE-C [101]

Figure 6.10: First 2 principal components of learned state representations of the $14 \times 14$ grid-world in Figure 6.8b. The color bar represents the reward.

actions, the latent policy outperforms the DQN policy in terms of convergence speed to the optimal solution. 


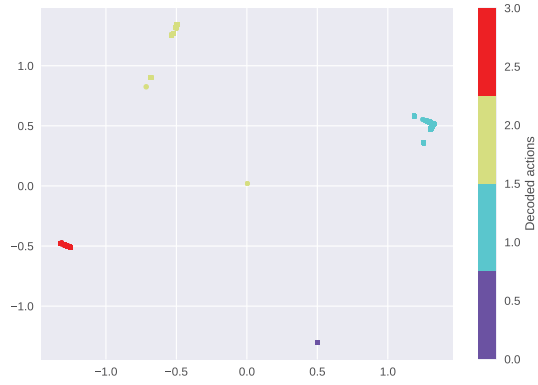

(a) Maze $6 \times 6$ - latent actions

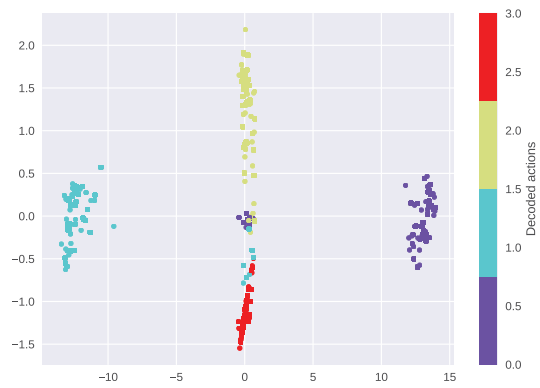

(c) Maze $6 \times 6$ - latent transitions

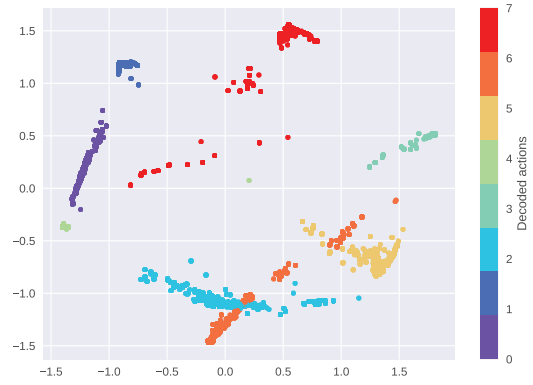

(b) Maze $14 \times 14$ - latent actions

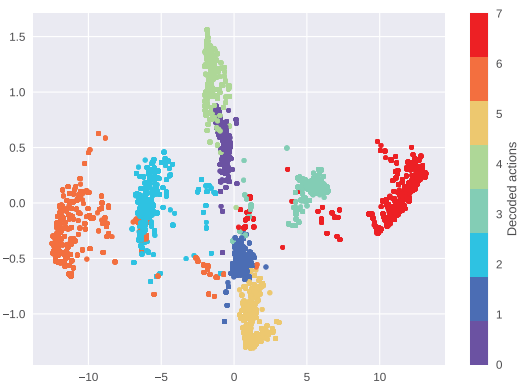

(d) Maze $14 \times 14$ - latent transitions

Figure 6.11: First 2 principal components of the learned action space $\overline{\mathcal{A}}$ and the $\Delta \overline{\mathrm{T}}$ in the different grid-worlds in Figure $6.8 \mathrm{a}-6.8 \mathrm{c}$. The color bar indicates the different discrete actions.

\subsubsection{Mobile Robot Navigation}

\section{Comparison of the Learned Representations}

The learned state representations obtained with the different approaches are shown in Figure 6.13. Similarly to the grid-world case, our approach can learn a valid state representation resembling the underlying state space in terms of smoothness and reward properties (i.e. distance to the target). This aspect can be noticed from the state distribution and its colour gradient in Figure 6.13a. It is worth highlighting the benefits of the contrastive loss (Equation (6.8)) for learning state representations. Especially in the case of an underlying continuous state space, such as in the robot navigation experiments, the methods employing a contrastive 


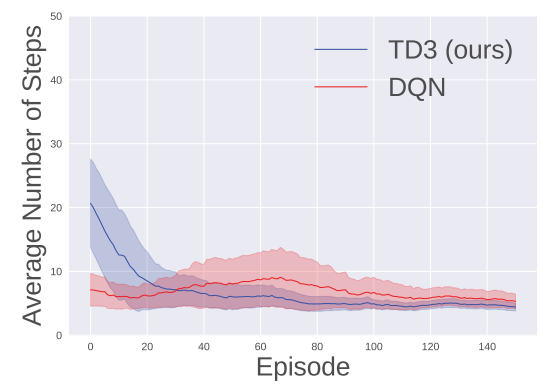

(a) Maze $6 \times 6-4$ actions

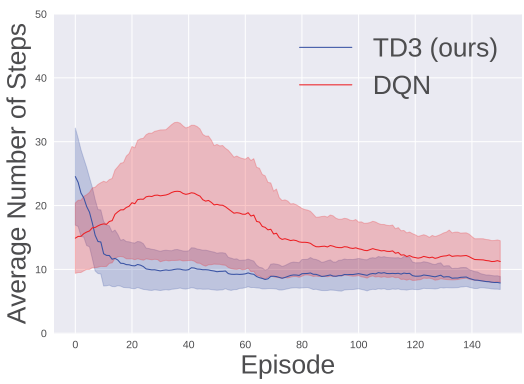

(b) Maze $14 \times 14-8$ actions

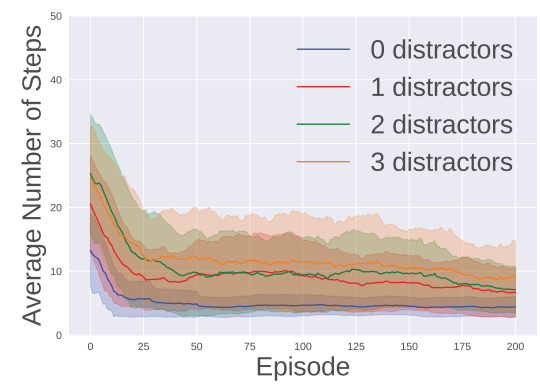

(c) Maze $5 \times 5-4$ actions, $0,1,2,3$ distractors

Figure 6.12: Average number of training steps. The solid line represents the mean and the shaded area, the variance of the best performing three random seeds out of ten.

loss tend to improve the quality of the learned representation, and this can be noticed from Figure 6.13a, 6.13b, and 6.13e.

\section{Comparison of the Learned Policies}

In Figure 6.14, the performance of the two agents is compared for the different action spaces. In both cases, the latent policy outperforms the DQN policy in terms of the average success ratio over training.

\subsection{Discussion and Future Work}

We presented a framework for the self-supervised learning of state and action representations for Reinforcement Learning for high-dimensional problems. Instead 


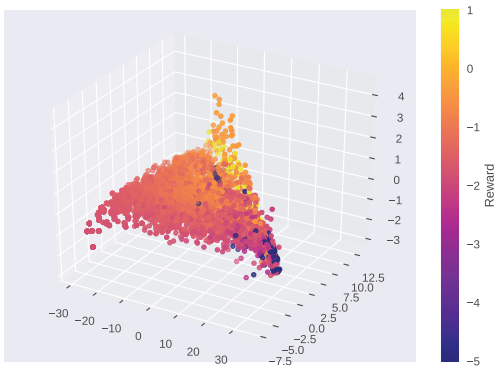

(a) Our

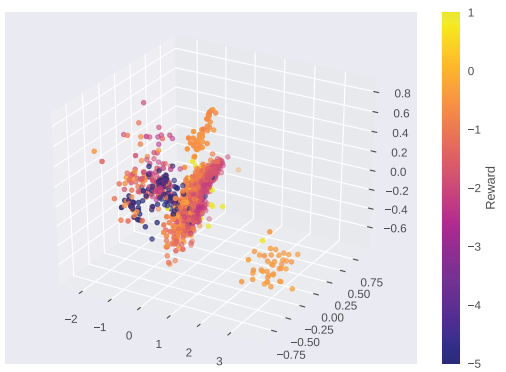

(c) D-MDP [62]

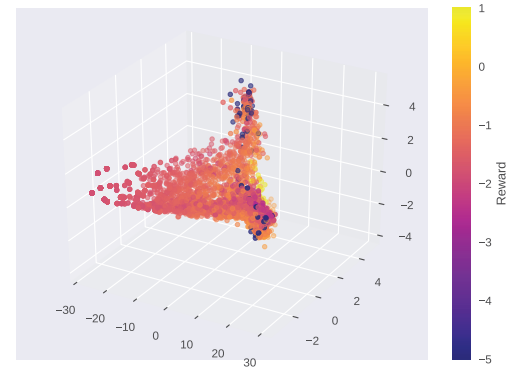

(b) MDP-H [87]

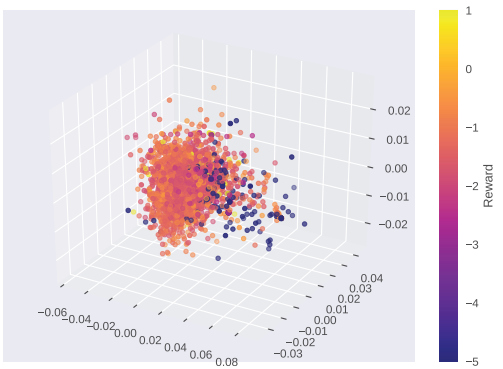

(d) JSAE [101]

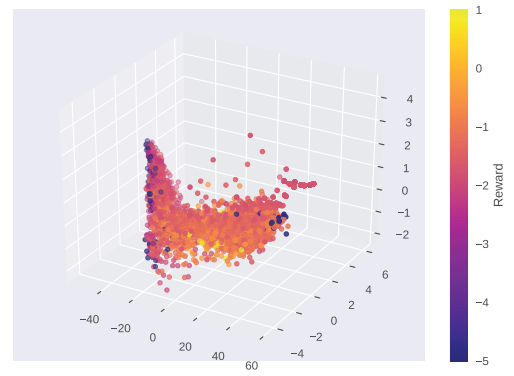

(e) JSAE-C [101]

Figure 6.13: First 3 principal components of learned state representations using the samples from the environment in Figure 6.9. The color bar represents the reward.

of learning the complex policy $\pi_{o}: \mathcal{O} \longrightarrow \mathcal{A}$ mapping the observation space $\mathcal{O}$ directly to the action space $\mathcal{A}$, using self-supervised objectives (Equation (6.6)-(6.8), 


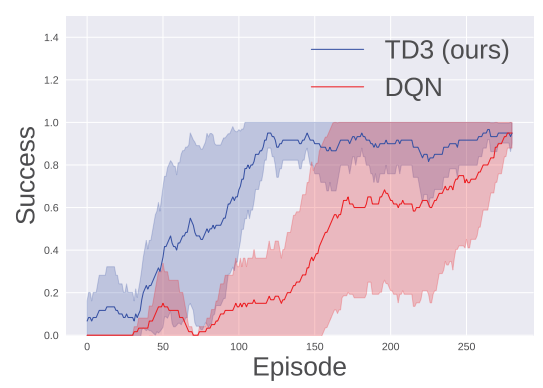

(a) Robot - 3 actions

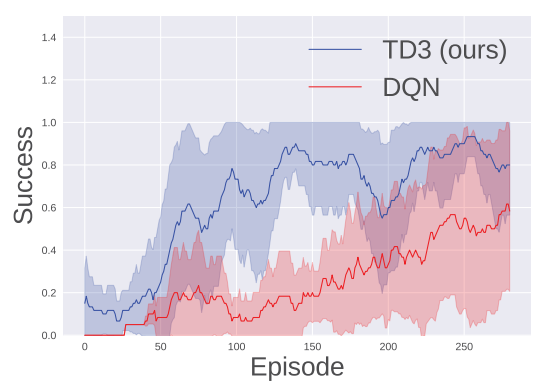

(b) Robot - 8 actions

Figure 6.14: Success ratio over training. The solid line represents the mean and the shaded area the variance of the best performing three random seeds out of ten.

(6.10)), we transform a (potentially) high-dimensional MDP $\mathcal{M}$ (either discrete or continuous) in a homomorphic, continuous, and low-dimensional $\operatorname{MDP} \overline{\mathcal{M}}$ in $\overline{\mathcal{S}}$ and $\overline{\mathcal{A}}$. The latent policy $\bar{\pi}: \overline{\mathcal{S}} \longrightarrow \overline{\mathcal{A}}$ is now a continuous policy, independent of the dimensions of the observation space $\mathcal{O}$ and the action space $\mathcal{A}$. Therefore, the method scales well with the dimension of the underlying true state, the observation space, and the action space. The latent policy can be quickly and efficiently optimised by any policy gradient algorithm. Moreover, because the policy is learned using a state representation, it is naturally more robust against noise, disturbances, and untrained features (see Figure 6.12c).

The proposed framework learns state and action representations through learning the MDP dynamics (transition and reward models). The framework directly combines model-free and model-based Reinforcement Learning. The learned latent transition and reward model can be used for sampling and planning. The balance between the use of the models, the real samples for exploration of the spaces and optimisation of the policy is an interesting future direction.

The action encoder model $\psi_{e}$ is trained to optimise two objectives (Equation (6.9), (6.10)), however, similarly to [107], if priors are available, it is possible to use them to shape the learned action space and consequently the whole state-action representation.

We have only considered simple one-to-one mappings $\psi_{e}$ and $\delta_{d}$ between actions and latent actions. However, it is possible to learn high-level action representations by learning many-to-one mappings. A sequence of actions is mapped to a single 
latent action, and the latent action is consequently decoded into a sequence of actions back. In this case, it is possible to rely on the semi-MDP [67] framework to learn a semi-MDP homomorphism. This aspect may have an impact in all the robotics applications with complex action spaces or in natural language processing.

Eventually, we have only considered the case of deterministic MDPs, but the framework can be extended to stochastic MDPs. Moreover, we have restricted the study to Markovian observation space so that we could rely on the MDP framework. However an important future step is to bring this framework to partiallyobservable MDPs (POMDPs), in which a single observation is not sufficient to unequivocally determine the agent's state. In this context, it is interesting to investigate the use of recurrent architectures and transformers [108].

\subsection{Conclusions}

In this chapter, we proposed a framework for state and action representation learning for Reinforcement Learning. Our approach transforms a given MDP $\mathcal{M}=\langle\mathcal{O}, \mathcal{A}, \mathrm{T}, \mathrm{R}\rangle$ into an homomorphic $\operatorname{MDP} \overline{\mathcal{M}}=\langle\overline{\mathcal{S}}, \overline{\mathcal{A}}, \overline{\mathrm{T}}, \overline{\mathrm{R}}\rangle$. The new MDP $\overline{\mathcal{M}}$ has continuous state and action spaces, but it is easier to solve using any policy gradient algorithms. We showed that the optimal latent policy $\bar{\pi}$ for $\overline{\mathcal{M}}$ is optimal for the original MDP $\mathcal{M}$ and that it can be efficiently and effectively learned. The optimal latent policy converges faster than the DQN agent trained on a state representation to the optimal solution as soon as the underlying true state and action spaces grow in size and complexity. 



\section{Chapter 7}

\section{Towards Autonomous Pipeline Inspection with Hierarchical Reinforcement Learning}

Inspection and maintenance are two crucial aspects of industrial pipeline plants. While robotics has made tremendous progress in the mechanic design of in-pipe inspection robots, the autonomous control of such robots is still a big open challenge due to the high number of actuators and the complex manoeuvres required. To address this problem, we investigate the usage of Deep Reinforcement Learning for achieving autonomous navigation of in-pipe robots in pipeline networks with complex topologies. We introduce a hierarchical policy decomposition based on Hierarchical Reinforcement Learning to learn robust high-level navigation skills. We show that the hierarchical structure introduced in the policy is fundamental for solving the navigation task through pipes and necessary for achieving navigation performances superior to human-level control. A video of our experiments can be found at:https://youtu.be/uyjSHulpGoI.

This chapter includes an adaptation of:

Nicolò Botteghi, Luuk Grefte, Mannes Poel, Beril Sirmacek, Christoph Brune, Edwin Dertien, and Stefano Stramigioli. Towards Autonomous Pipeline Inspection with Hierarchical Reinforcement Learning. Submitted to International Conference on Robot Intelligence, Technology and Applications, 2021.

The Section Background was removed as treated in Chapter 2 and 3, and the notation adapted to be consistent with the notation introduced in this thesis. 


\subsection{Introduction}

Pipelines networks are the fulcrum of the oil and gas industries and of gas and water mains. These pipes must be periodically inspected to guarantee the safety and proper functioning of the plants. However, inspection is usually a long, expensive and tedious procedure that requires the shut-down of the whole plant and, in the specific case of industrial pipelines, the removal of the insulation around the pipes. With metal pipes, the inspection is currently performed from the outside using ultrasonic or magnetic probes that measure the wall thickness. Unfortunately, these inspection methods provide limited information about the state of pipes due to very low resolution and noisy data and require the removal of the insulation. Currently, Pipeline Inspection Gauges, or PIGs, are used to inspect the pipelines from the inside. However, while PIGs do not require a full shut down of the plant, they cannot be used to inspect networks with complex topologies, e.g. sharp corners, T-junctions and vertical sections.

In the last two decades, inspection robotics has focused on designing new robotic prototypes for in-pipe inspection. However, especially in the case of small diameter pipes, the mechatronics of these robots is complex, costly and with low operability [7]. While the design of these in-pipe robots has quickly progressed, many steps have yet to be taken to navigate and inspect complex pipes autonomously. In-pipe inspection robots operate in highly constrained environments, with limited sensing equipment, without or with limited knowledge of the pipeline-network structures beforehand and unpredictable situations due to the contact dynamics and slippage as different fluids and media can be present in the pipes during the inspection. Furthermore, these robots are often composed of multiple joints, and multiple actuators have to be simultaneously controlled, making the design of an autonomous, robust and adaptable navigation system challenging.

Reinforcement Learning [1], or RL, has proven to be a valuable solution for many robotics challenges and tasks such as mobile robot navigation, dexterous manipulation through robotic arms, and bipedal robot locomotion [10]. However, when the task requires the execution of a sequence of complex skills on a long temporal horizon, Reinforcement Learning algorithms tend to struggle [109].

Hierarchical Reinforcement Learning, or HRL, takes advantage of the hierarchical policy decomposition to exploit underlying problem structures and simplify 
the learning of complex tasks. The hierarchical decomposition can be either defined by using prior knowledge [67], [110], [111], [112], or can be automatically learned during training [109], [100], [113]. While the latter category of algorithm does not require expert knowledge for defining the hierarchy, the autonomous discovery of the options often leads to sub-optimal policies if additional regularisers are not used during the learning phase [111], [113].

We propose a Hierarchical Reinforcement Learning framework for autonomous navigation of multi-actuated, complex robots for in-pipe inspection. This is shown in Figure 7.1. In particular, we focus on the pipe inspection robot called PIRATE

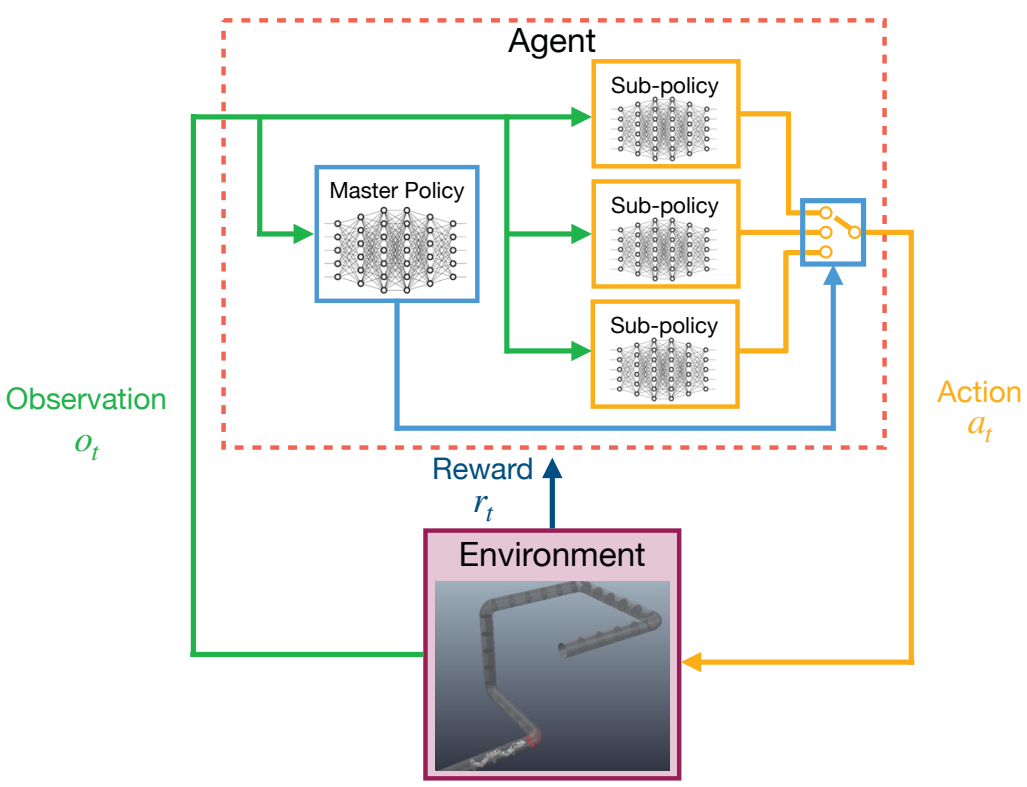

Figure 7.1: Proposed hierarchical Reinforcement Learning architecture for the autonomous navigation of the PIRATE robot.

[114], but the approach can be easily adapted to many different snake-like pipe inspection robots. The framework combines expert knowledge for determining the hierarchy of the policies with simple auxiliary reward functions for ensuring the optimal behaviour of the sub-policies. To the best of the authors' knowledge, this is the first time a robust and flexible control solution employing Reinforcement Learning for the autonomous navigation of snake-like pipe inspection robots with a clamping mechanism is presented. In this chapter, we aim at addressing the 
following research questions:

1. What is a good hierarchy for learning robust and generalisable policies for the pipeline inspection robot PIRATE?

2. What are the benefits of such a hierarchical decomposition of the policy?

3. How do the learned policies compare to human-expert control?

\subsection{Related Work}

Exploiting structures and hierarchies is one of the most important challenges for scaling Reinforcement Learning algorithms to more complex real-world problems. A famous Hierarchical Reinforcement Learning approach is the so-called Option framework [67], [98]. Options are temporally extended abstract actions corresponding to the set of skills the agent need to learn in order to solve tasks. In this framework, above the options, we always find a high-level policy, learning to select the best option for the given context. Options can be either hand-crafted based on prior knowledge, as in [110], [111], [112], or can be automatically learned during training, as in the option-critic framework proposed by [113]. In the latter case, however, regularization, e.g. entropy maximisation, has to be often employed to prevent the learning of sub-optimal options.

A different approach is followed by HIRO [109], and FuN [100], where the high-level policy does not select anymore which abstract action to actuate, but, instead, determines abstract goals for the low-level policies. Similar to the option framework, the abstract goals are chosen with a lower frequency than the actions chosen by the low-level policies.

In our work, we want to exploit the high amount of prior knowledge we have about the robot's mechanics and motion and about the navigation task in the structured pipeline networks. Therefore, we find the option framework the most suitable for this scenario.

\subsection{Autonomous Pipeline Inspection Robots}

In the last two decades, many innovative and different designs of in-pipe inspection robots have been proposed. According to [115], most of these robots use wheels 
for locomotion, have modular and snake-like bodies, and clamp inside the pipes. These three elements, when combined, allow the maximum flexibility of usage in different pipeline structures with vertical sections, junctions and corners.

To the category of modular and snake-like robots belongs MAKRO [116], a sewer inspection robot with an articulated body and multiple wheels. This robot, however, cannot clamp itself in the pipes, which significantly reduces the range of its usage. Another example is the robot proposed in [117] for inspection of urban gas pipelines. The robot has complicated mechanics with many joints and wheels, and it can clamp inside the pipe, allowing travelling even vertical sections. In [118] a bio-inspired snake robot is designed and presented. However, while the control principle is introduced, no actual test in complex pipes is shown. Eventually, the PipeTron [119] and the PIRATE [114] are snake-like robots with wheel-based locomotion and the ability to clamp inside the pipes. These features allow these two robots to be very flexible in terms of the range of use and functionalities.

Differently from the previously cited works, KANTARO [120] employs wheels for locomotion. Its body is simple, allowing easier movements through junctions and reductions of the pipe diameters. The motion of this robot is, however, limited to planar pipeline networks without vertical sections. A similar design is used in [7] with the addition of a traction system for improving locomotion.

While the designs of these robots have greatly progressed in the past year, autonomous inspection is still a big open challenge [116]. Especially for the complex snake-like robots with clamping mechanisms, the control and navigation are very complicated, and no fully autonomous and robust solutions for navigating complex pipeline networks with sharp corners and vertical sections are yet present.

\subsubsection{PIRATE Robot}

The PIRATE robot [114] is designed to travel through pipes having different diameters, vertical sections and sharp corners. The PIRATE robot has six actuated joints $J_{1, \ldots, 6}$ and six actuated wheels $w_{1, \ldots, 6}$, as shown in Figure 7.2. The robot is composed of two (inverted) $\mathrm{V}$-shape sections $\left(w_{1,2,3}\right.$ and $\left.w_{4,5,6}\right)$ that allow its clamping inside the pipes. Each joint and wheel can be either controlled through position or velocity set-points fed to low-level (PID) controllers running on embedded boards. The robot is equipped with absolute encoders for measuring the 


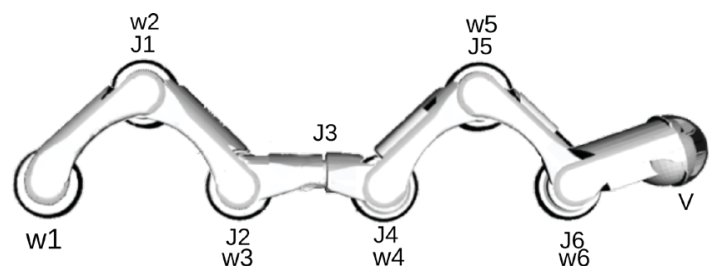

Figure 7.2: Schematic drawing of the PIRATE robot. $w_{i}$ indicates the $i^{t h}$-wheels, $J_{i}$ the $i^{t h}$-joints and $V$ the vision sensor.

joints and wheels' rotation and with inertial measurement units (IMU) for measuring acceleration and orientation. Moreover, the robot perception is enhanced with cameras for visual inspection and with Light Detection and Ranging (LiDAR) sensors for navigation.

\subsection{Methodology}

\subsubsection{Reinforcement Learning for the PIRATE Robot}

\section{The Action Space}

The action space $\mathcal{A} \in \mathbb{R}^{12}$ is chosen to be continuous to be able to execute smoother and more advanced manoeuvres. For this reason, the Reinforcement Learning algorithm chosen is PPO, similarly to [112]. Each wheel $w_{1, \ldots, 6}$ and each clamping joint $J_{1,2,4,5}$ are controlled using velocity commands, while the rotational joint $J_{3}$ and the vision-sensor joint $J_{6}$ are controlled using position commands, as shown in Table 4.2. In our experiments, we found beneficial the position control of the rotation joint $J_{3}$ and the vision-sensor joint $J_{6}$ for achieving more accurate motion. These two joints are critical for the orientation procedure of the robot with respect to corners ${ }^{1}$, and for their detection, respectively.

\begin{tabular}{||c|c|c||}
\hline Control Mode & velocity & position \\
\hline Action space & $J_{1,2,4,5}, w_{1, \ldots, 6}$ & $J_{3}, J_{6}$ \\
\hline
\end{tabular}

Table 7.1: Control modes for the PPO policy, velocity corresponds to the velocity control of the actuators, while position to the position control.

\footnotetext{
${ }^{1}$ Due to its mechanics, the PIRATE robot requires a specific relative orientation with respect to the pipe corners in order to navigate through it.
} 


\section{The State Space}

We compare and analyse two alternative state spaces: the kinematic and the visual. The kinematic state-set includes all the kinematic information such as the positions $q_{J_{1, \ldots, 6}}$, orientations $o_{J_{1, \ldots, 6}}$ and velocities $v_{J_{1, \ldots, 6}}$ of the joints in the space, the positions $p_{w_{1, \ldots, 6}}$ and velocities $v_{w_{1, \ldots, 6}}$ of the wheels in the space, and the previous actions taken $a_{t-1}$. The visual state-set extends the kinematic one with external perception, i.e. $3 \mathrm{D}$ depth images $i_{d}$.

It is worth mentioning that the absolute positions, orientations of the robot in the space can be estimated, for example, by means of any Simultaneous Localization and Mapping algorithm [121]. However, for the sake of simplicity, we assume to have a good estimate available. Moreover, wheels and joints relative positions and velocities can be measured using the onboard sensors (encoders and IMUs).

\section{The Reward Function}

For a generic inspection task, the goal is to inspect as much of the pipeline as possible. Thus the reward function that the agent tries to maximise is proportional to the forward distance travelled by the robot. The reward function is shown in Equation (7.1).

$$
\mathrm{R}(s, a)=d_{t}-d_{t-1}
$$

where $d_{t}$ corresponds to the absolute position of the robot at time-step $t$ and $d_{t-1}$ to the position at time-step $t-1$ with respect to the origin of the chosen reference frame. This reward function encourages the agent to drive the robot forward in the pipeline.

\subsubsection{Hierarchical RL and Policy Decomposition}

While the task of learning to navigate might be easy for differential-drive mobile robots, for complex multi-actuated robots in constraint environments, this is not the case. In particular, moving through a straight section or a corner of the pipeline networks requires complicated coordination of actuators over long sequences of atomic actions. However, the high-level control is relatively straightforward, and all pipe inspection robots share the same needs of either travelling through straight pipes or moving through corners and junctions. We can exploit such a structure by hierarchically decomposing the Reinforcement Learning policy into a Master 


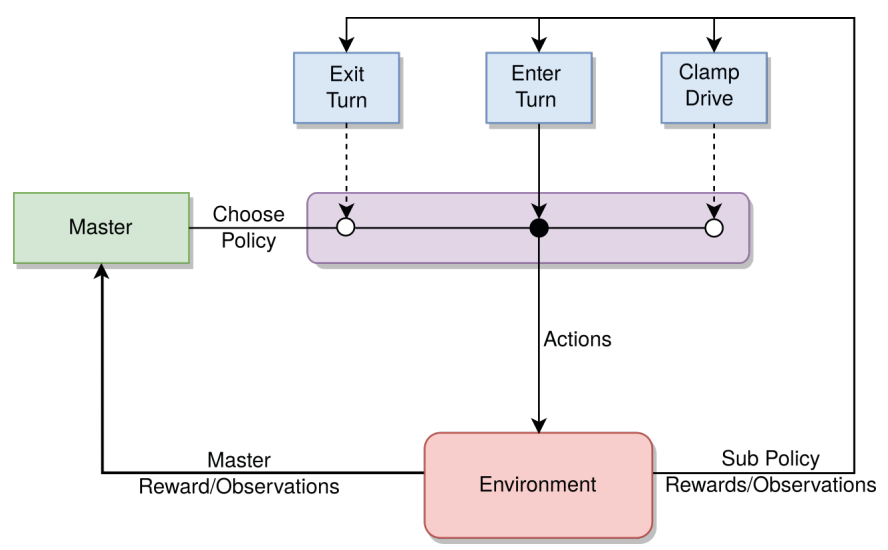

Figure 7.3: Hierarchical decomposition of the policies.

policy and three sub-policies.

\section{Master Policy and Sub-Policies}

We introduce a two-level hierarchical policy architecture, inspired by the Option framework [67], where the Master policy chooses which of the three sub-policies to deploy to maximise the reward function, in Equation (7.1). Each sub-policy corresponds to a specific skill the agent requires to navigate through the pipeline networks, namely clamping and driving, and entering and exiting a corner, as shown in Figure 7.3.

The Clamp Drive sub-policy is responsible for clamping the robot in the pipes and drive through straight sections. Due to the robot's mechanics, driving and clamping are dependent actions, and the robot cannot travel any pipe without first clamping its body in it. The Enter Turn sub-policy is in charge of driving the first V-shape of the robot through the corner, while Exit Turn completes the turning procedure and clamps the robot in the new pipe segment, as shown in Figure 7.4. The turning procedure is the most challenging to learn. Thus we split this task into two different skills.

The Master policy tries to learn to choose the correct abstract action, or skill, for the circumstance. This policy receives observation only when a specific subpolicy has finished its execution, i.e. after 30 time-steps or when an early stopping condition is met (e.g. when the goal is reached) in our case. Because the Master policy picks action with a lower frequency than the sub-policies, it is even more 


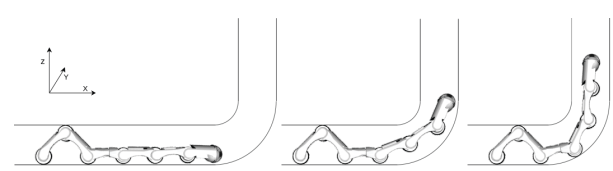

(a) Enter Turn

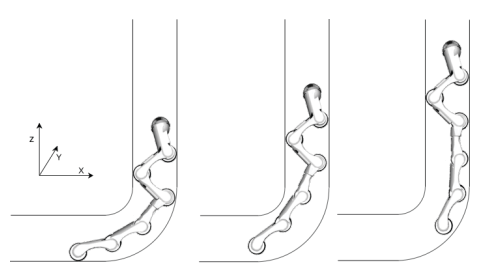

(b) Exit Turn

Figure 7.4: Motion of the PIRATE robot through a corner.

important that such policy receives good observations from the environment. Otherwise, it is not able to choose the best sub-policy to enable. For example, if the depth camera is pointing at the ceiling or the bottom of the pipe, and no clear view of the pipe is present, the Master policy has no way to know if the robot is close to a corner and what is its orientation. However, the hierarchical decomposition of the task allows us to define auxiliary reward functions for each of the sub-policies that can speed up the learning of the skills and consequently of the task. This is discussed in Section 7.4.2.

Another advantage of the policy decomposition is the possibility to constrain the action spaces of each sub-policy. This decomposition, again, allows learning better policies more efficiently. The choice of the different action spaces is shown in Table 7.2. While the Clamp Drive policy keeps the action space defined in Table 7.1, while the Enter Turn and Exit Turn disable the front-wheels rotation and back-wheel rotation respectively. These wheels are not needed for moving through corners and junctions. Moreover, the control mode of joints is switched to position control, except for joints $J_{1}$ and $J_{5}$ respectively $^{2}$, for more accurate manoeuvring.

\begin{tabular}{||c|c|c|c||}
\hline Control Mode & velocity & position & $N / A$ \\
\hline Clamp Drive & $J_{1,2,4,5}, w_{1, \ldots, 6}$ & $J_{3}, J_{6}$ & - \\
Enter Turn & $J_{1}, w_{1,2,3}$ & $J_{2, \ldots, 6}$ & $w_{4,5,6}$ \\
Exit Turn & $J_{5},, w_{4,5,6}$ & $J_{1,2,3,4,6}$ & $w_{1,2,3}$ \\
\hline
\end{tabular}

Table 7.2: Action space and control modes for the different sub-policies. velocity corresponds to velocity control of the actuators, position to position control and $N / A$ if the actuator is not used by the sub-policy.

\footnotetext{
${ }^{2}$ The joints $J_{1}$ and $J_{5}$ need to hold the clamping of their $\mathrm{V}$-shapes of the robot, and in our experiments, we have discovered velocity control mode optimal for such task.
} 
This choice of hierarchy is driven by the nature of the task, the robot's knowledge, and the topology of the pipeline networks. However, it can be easily adapted to different pipe inspection robots, given the similarities of tasks and mechanics of such robots. The three sub-policies define three different high-level skills the agent has to learn to navigate any pipe. In general, one could think of lower-level sets of skills, such as, for example, two independent clamping and unclamping policies for the V-shapes or one policy for actuating all the wheels independently of the joints. However, this would be detrimental to the overall performance. Due to the robot mechanics, most of the low-level atomic skills are dependent on each other, e.g. if both $\mathrm{V}$-shapes are clamped, the PIRATE robot cannot actuate the joint $J_{3}$. Thus the Master policy would need to learn an even more complex sequence of commands. Moreover, with a finer discretisation of the skills, the Master policy task would become more complex as it is harder to distinguish which skill to use in each state. The proposed hierarchy trades off the task-complexity for the Master policy and the sub-policy.

\section{Auxiliary Reward Functions for the Sub-Policies}

To specialise the sub-policy and to quickly learn the skills, we define a clamping reward that promotes clamping by penalising the distance between the wheels:

$$
R_{\text {clamping }}=-\left\|p_{\mathrm{w}_{1}}-p_{\mathrm{w}_{3}}\right\|_{4}
$$

where $p_{\mathrm{w}_{1}}$ and $p_{\mathrm{w}_{3}}$ are the positions of the first and third wheel of the robot (see Figure 7.2). This is particularly useful in the Clamp Drive policy.

Additionally, we employ a depth-camera reward for promoting the proper orientation of the depth camera in the direction of the pipe axis to obtain richer information about the pipe network. This is shown in Equation (7.3)

$$
R_{\text {depth }}=\operatorname{std}(i)
$$

where $s t d$ is the standard deviation and $i$ is the depth image ${ }^{3}$.

\footnotetext{
${ }^{3}$ When facing a wall, the images contain points with similar distances, thus with low standard deviation. Conversely, when properly aligned with the pipe axis, the images have higher standard deviation.
} 


\subsection{Experimental Design}

\subsubsection{Autonomous Navigation in Pipeline Networks}

The goal of the experiments is to learn an optimal navigation policy for manoeuvring the PIRATE robot in an unknown a priori pipeline network. In this work, we focus on the navigation problem in pipeline networks composed of straight sections and $90^{\circ}$ bends with different smoothness, either $1 \mathrm{D}$ and $2 \mathrm{D}^{4}$. We assume that the agent does not know the position of the end-point of the inspection mission, but it can detect it once the robot reaches it. This scenario represents a generic inspection mission. The robot has to autonomously navigate until a problem, e.g. a crack or corrosion in the pipe wall and a consequent reduction of the wall thickness, is found.

We train and test the agents in a static pipeline, i.e. the pipeline is not changing over training nor testing. In a dynamic pipeline, i.e. the pipeline network is randomly changing configuration in each training episode. The possible changes occurring in the pipeline are changes in the direction of the corners and relative distance among them.

\subsubsection{Simulation Environment}

We test the approach in the simulation environment V-REP [122] using a model of the PIRATE robot. V-REP allows for realistic physical simulations and allows learning transferrable policies to real robots [123]. Bullet physical engine [124] is used. Moreover, the Ray framework and the RLlib library [125] are used for developing the HRL framework. OpenAI Gym [126] support is available in RLlib, and this is used to build the RL environment. OpenAI Gym provides a framework to build a custom RL environment. RLlib communicates with V-REP by using PyRep [105]. Furthermore, RLlib uses the TensorFlow library for building neural network models. The PPO implementation of RLlib is used for training the agents.

\subsubsection{State Space and Neural Network Architectures}

We first study the effect of the two different state-sets, introduced in Section 7.4.1, on the performances of a single PPO agent, with action space defined in Table 7.2,

\footnotetext{
${ }^{4}$ Pipe corners are classified by indicating the smoothness of the corner with respect to the diameter of the pipe D.
} 
when trained on a static and a dynamic pipe configuration.

\subsubsection{Hyperparameters Tuning}

Three independent grid-search experiments are performed to find a good set of hyperparameters. This is shown in Table 7.3.

\begin{tabular}{||c|c|c||}
\hline Experiment 1 & Experiment 2 & Experiment 3 \\
\hline Learning rate & Clip, Lambda, Entropy-coefficient & Train-batch-size, Mini-batch-size \\
{$\left[1 \mathrm{e}-4,1 \mathrm{e}-5^{*}, 1 \mathrm{e}-6\right]$} & {$\left[0.1,0.2^{*}\right],\left[0.99^{*}, 0.95\right],\left[0.005^{*}, 0.01\right]$} & {$\left[500,1000^{*}, 2000\right],\left[300^{*}, 100\right]$} \\
\hline
\end{tabular}

Table 7.3: Hyperparameters ranges used in the grid-search experiments. * indicates the value used in the experiments presented in the paper.

\section{Network Architecture}

In the case of the kinematic state-set, the policy and the value function networks share a single fully connected layer of dimension 128 with tanh activation. The features are then fed to two identical branches composed of two fully connected layers of dimensions 128 and 64, respectively, with tanh activations. The output layer for the policy network has the dimension of the action space, while it has dimension 1 for the value function network. This architecture is shown in Figure 7.5 .

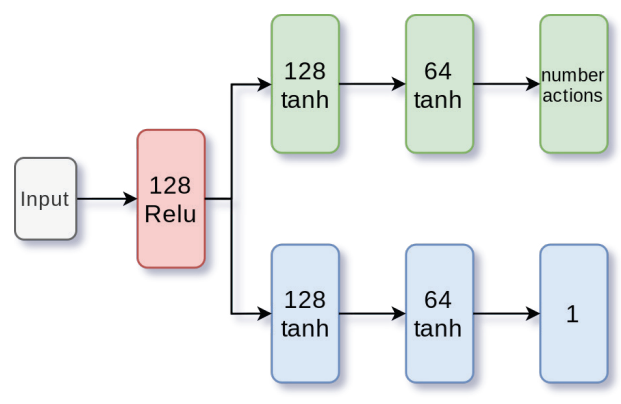

Figure 7.5: Neural network architecture for the kinematic state-set.

When the visual state-set is employed, the depth images are initially separated from the kinematic information of the state vector and pre-processed by two convolutional layers, with five and ten filters, respectively, of size $5 \times 5$, and stride two, and by a fully-connected layer. These features, extracted from the depth images, 
are then concatenated to the other elements of the state vector and fed to the same architecture used by the kinematic state-set. This is shown in Figure 7.6. A similar architecture is successfully employed in [123].

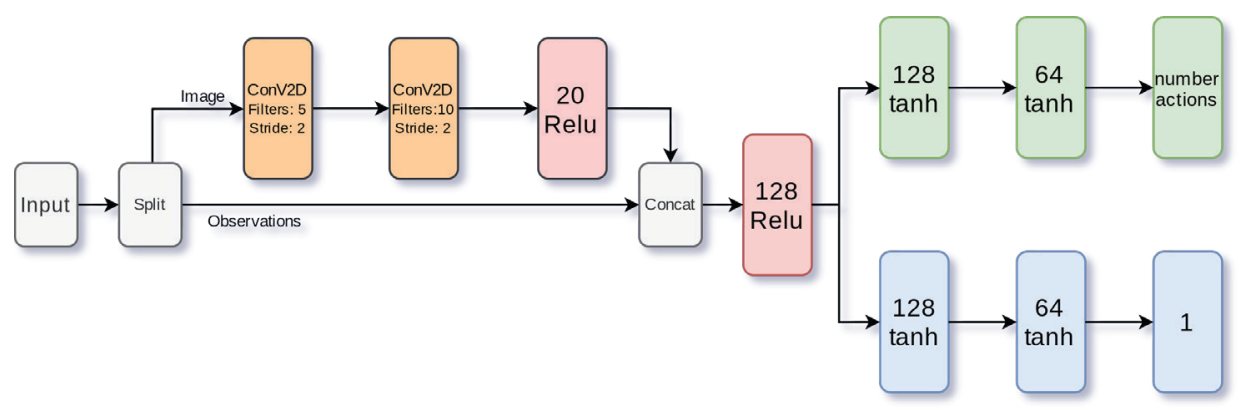

Figure 7.6: Neural network architecture for the visual state-set.

In the case of the visual state-set, we also study the use of a recurrent architecture, adding a single LSTM layer to the visual architecture, after the concatenation of the features from the depth images and the other components of the state vector, as shown in Figure 7.7.

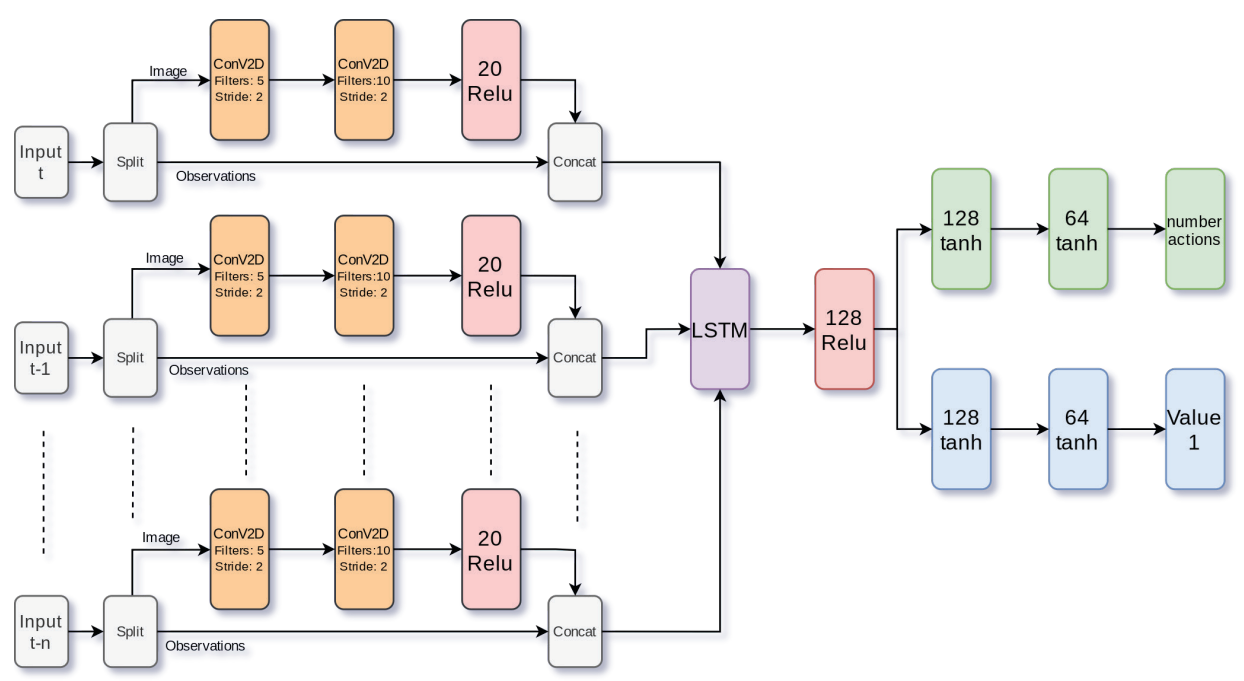

Figure 7.7: Recurrent architecture for the visual state-set. 


\subsubsection{Comparison of RL and HRL}

To assess the value of the hierarchical policy architecture, we compare the single PPO agent with the HRL agent trained proposed hierarchical approach on both static and dynamic pipeline networks using the visual observation group.

\section{Training Regime of the HRL Method}

We test two different training approaches for the HRL architecture: simultaneous optimisation of the four policies and independent optimisation of the policies. In simultaneous optimisation, the Master policy and the sub-policies are optimised at the same time in the same environment. In contrast, in the independent optimisation procedure, the sub-policies are first trained in specialised environments ${ }^{5}$ and, only once optimised, the master policy is trained.

\subsection{Results and Discussion}

\subsubsection{Different Observation Groups}

We first present the results obtained with the single PPO agent (SRL) with the two different observation groups in the static and dynamic environments. For each observation group, four independent experiments are performed, and the results are shown in Figure 7.8.

While the visual state set is sufficient for completing the $\operatorname{task}^{6}$ of navigating a three-junction pipe in the static environment, see Figure $7.8 \mathrm{a}$, it is not enough in the dynamic case in which the three junctions randomly change position and orientation each training episode, as shown in Figure 7.8b. The agent observing the depth images outperforms the one relying only on the kinematic information. Thus, we can conclude that it is not possible to learn a policy that drives the robot through the pipes by only looking at the kinematic information. Moreover, in the dynamic case, the kinematic state-set generates catastrophic forgetting, as shown in Figure 7.8b, where the performances of the policy start decreasing the more the training goes on. Surprisingly, in the dynamic environment with only three

\footnotetext{
${ }^{5}$ For example, a specialised environment for the Clamp Drive sub-policy is composed of only straight pipes.

${ }^{6}$ Considering the length of the pipes, a cumulative reward value above 35 means that the task is solved.
} 


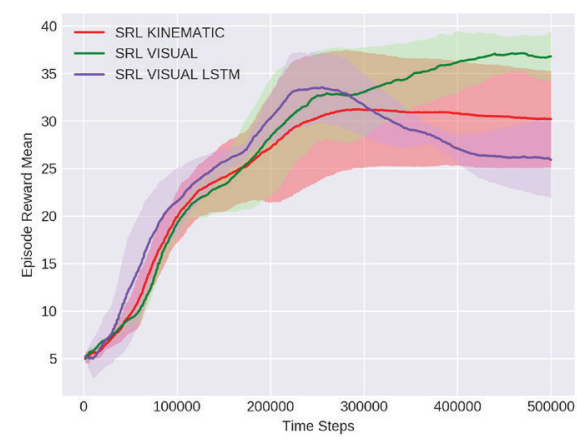

(a)

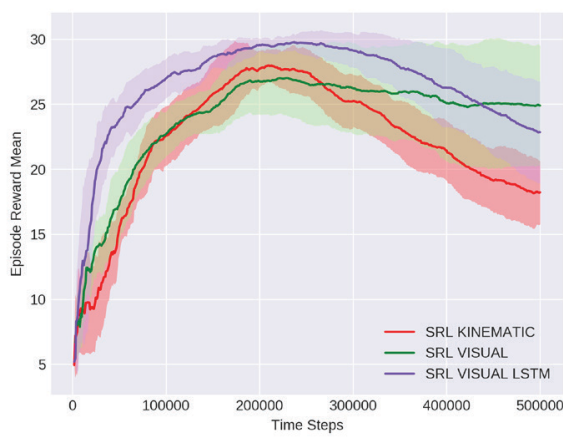

(b)

Figure 7.8: Cumulative reward during training of the PPO policy with different observation groups in the case of static environment (Figure 7.8a) and dynamic (Figure $7.8 \mathrm{~b}$ ). The solid line represents the average cumulative reward, while the shaded area its standard deviation.

junctions, even the visual state-set does not allow sufficient policy improvements to reach the end of the pipe. The single PPO agent cannot learn a robust policy to deal with a random pipe-network configuration. Eventually, the visual policy with LSTM does not improve the performances either in the static case or in the dynamic one. By including information on the positions and velocities of all the wheels and joints and depth information about the pipe network, the state vector already contains all the information needed to determine the state of the environment and, consequently, choose the best actions.

\subsubsection{Comparison of the RL and HRL Performances}

We now present the results obtained using the HRL framework proposed in Section 7.4.2 in a dynamic five-junctions pipe network. In particular, we compare HRL with pre-training of the sub-policies in different and specialised environments (HRL VISUAL) with the joint optimisation of all the policies (HRL SIMUL), as described in Section 7.5.5, and the single PPO (SRL VISUAL). The visual state-set is used by all the agents. The average cumulative rewards obtained during training in the five-junctions dynamic environment are shown in Figure 7.9a. Again, four independent experiments are performed for each curve in the plot. Both HRL agents outperform PPO, but while HRL VISUAL consistently solves the task, HRL SIMUL struggles to achieve the same performance. The independent training of 
the sub-policies has a positive influence on the overall performances of the HRLagent.

After the training phase, the performances of the agents are evaluated in unseen a priori environments. In Figure $7.9 \mathrm{~b}$, we show the evaluation in a challenging and randomly generated pipeline with five junctions. We report in Table 7.4 the distance travelled in meters by the different agents.

The single PPO agent (SRL VISUAL) can steer the robot only through the first corner and cannot advance more. The second corner requires the use of the rotation joint $\left(J_{3}\right)$ to orient the front $\mathrm{V}$-shape of the robot in the correct direction, but the PPO agent struggles to learn that. The proper re-orientation of the front is found one of the critical and most challenging manoeuvres for advancing through the pipe corners. The HRL agents perform better than PPO, but while the HRL with pretraining (HRL VISUAL) can steer the robot through all the five junctions, the HRL with simultaneous optimisation (HRL SIMUL) cannot make the robot escaping the fourth corner. The fourth corner requires great precision and robustness for each sub-policy, as it requires the re-orientation of the front modules of the robot while travelling upside down. This manoeuvre is more challenging to achieve when jointly training all the policies together. Again the policy with LSTM does not seem to improve the performance compared to the non-recurrent policy. A video of our experiments can be found at: https://youtu.be/H9IxZ1NYga4.

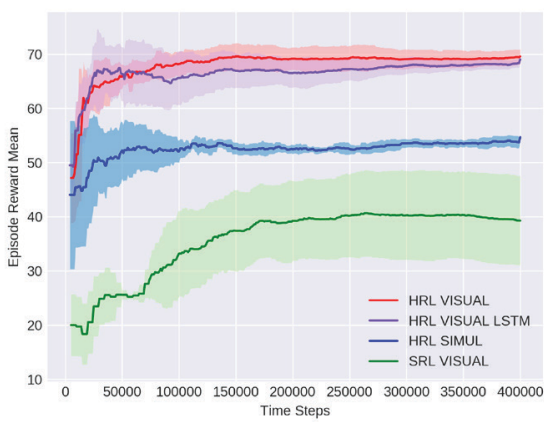

(a)

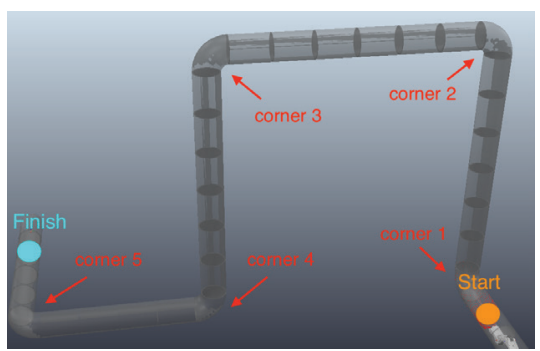

(b)

Figure 7.9: Comparison of HRL with sub-policy pre-training (red), HRL with joint optimization of the sub-policies (blue) and single PPO agent (green). The training results are shown in Figure $7.9 \mathrm{a}$ where the average reward (solid line) and the standard deviation (shaded area) are presented. The evaluation pipeline network is shown in Figure 7.9b. 


\subsubsection{Comparison with Human Control}

The PIRATE robot can be driven through the pipes by a human operator [114], but only in the case of transparent pipes that allow the operator to see the robot constantly. However, this operation is not practical in real scenarios when the operators can only rely on onboard sensors to control the robot.

To show the benefits of the use of Reinforcement Learning for controlling such pipe inspection robots, we try to manually control the robot in the same task of navigating through the pipe in Figure 7.9b. For fairness of comparison, the operator cannot see through the pipes and can rely on the same action space of the RL agents (Table 7.1) and the information contained in the visual state-set, i.e. depth-camera images and kinematic information.

When relying only on depth-camera images and kinematic information, a human struggles to complete the five-junctions task, in Figure 7.9b, even after many trials. In Table 7.4 we record the best trails. It is worth mentioning that a human operator may eventually solve the task for a fixed pipeline configuration. However, in the general inspection settings, the pipe configuration can change depending on the inspection task and can be even more complex. Thus, the human control of the robot does not seem a viable solution in this context.

\begin{tabular}{||c|c||}
\hline Method & Dist. traveled $(\mathrm{m})$ \\
\hline SRL VISUAL & 2.02 \\
HRL SIMUL & 4.83 \\
HRL VISUAL & $6.60^{*}$ \\
HUMAN & 4.88 \\
\hline
\end{tabular}

Table 7.4: Distance travelled in meters by different approaches in the evaluation environment in Figure 7.9b. Only the HRL with pre-trained sub-policies (HRL VISUAL) completes the task.

\subsubsection{Transferring the Policies to the Real Robot}

Because the experiments are conducted in simulated environments, the transfer of the learned policies to the real robot is an important aspect that has to be further investigated in future work.

The proposed HRL framework has proven to be robust against perturbations of the observations, e.g. additive noise on sensory readings, and can generalize 
to different and untrained pipe configurations. Moreover, while earlier research showed that policies, learned in virtual environments, relying on RGB images, are troublesome to transfer to the real-world [127], in our experiments, the agents only use a depth camera to perceive the environment and onboard sensory readings (encoders and IMUs) to obtain information of the robot's kinematic. Eventually, the agents' actions are position and velocity set-points for the robot's actuators. These set-points are then fed to low-level controllers that are in charge of tracking such reference values. By avoiding direct torque control of the actuators and by assuming well-tuned low-level controllers with similar performances to the one present on the actual robot, the difference in the dynamics between simulation and real-world is mitigated [10].

\subsection{Conclusions}

In this chapter, we presented a two-layers HRL framework for autonomous navigation of the snake-like pipe inspection robot PIRATE in pipeline networks with different topologies. We showed that the proposed hierarchical decomposition of the policies is necessary for solving the navigation problem of such robots in pipes with multiple junctions and with random configurations. The hierarchical decomposition allows learning a robust set of skills, and it is crucial for the generalization of the policy to unseen a priori pipeline networks. Moreover, with the proposed decomposition, the Master policy can learn different inspection missions if retrained with a different reward function and without the need of retraining the sub-policies. Eventually, when the sub-policies are pre-trained separately in specialised environments, the hierarchical framework outperforms human-controlled operations. 


\section{Reward Shaping}

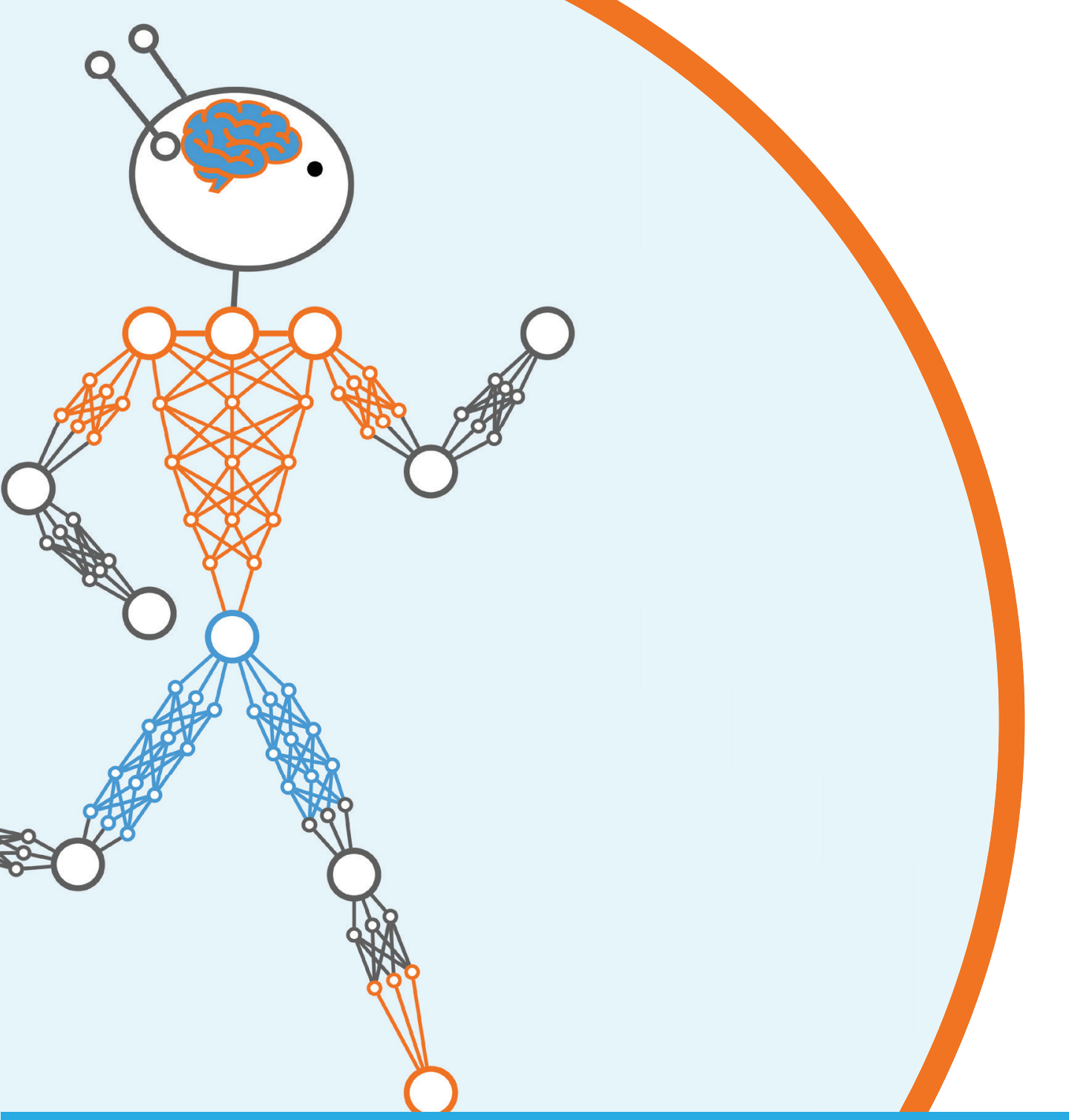





\section{Chapter 8}

\section{Entropy-Based Exploration for Mobile Robot Navigation: A Learning-Based}

\section{Approach}

In this chapter, we study the problem of reward shaping for autonomous robot navigation in unknown and indoor environments. We present a Deep Reinforcement Learning-based path planner for mobile robots, through continuous velocity commands, directly from raw sensory data for solving navigation tasks in different indoor environments. Here, we focus on shaping the reward functions to achieve faster training speed, better performances, and higher generalisation. We study the problem of transfer learning of such policies, learned in a simulated virtual environment, to a real robot without further retraining using real-world data. A video of our experiments can be found at: https://youtu.be/QWRyocjQzzW.

This chapter includes an adaptation of:

Khaled Mustafa, Nicolò Botteghi, Beril Sirmacek, Mannes Poel, and Stefano Stramigioli. Towards Continuous Control for Mobile Robot Navigation: a RL and SLAM Based Approach. International Society for Photogrammery and Remote Sensing Congress, 2019.

Nicolò Botteghi, Beril Sirmacek, Khaled Mustafa, Mannes Poel, and Stefano Stramigioli. On Reward Shaping for Mobile Robot Navigation: A Reinforcement Learning and SLAM Based Approach. arXiv, 2020.

Nicolò Botteghi, Khaled Mustafa, Beril Sirmacek, and Mannes Poel. Entropy-Based Exploration for Mobile Robot Navigation: A Learning-Based Approach. Planning and Robotics Workshop at International Conference on Automated Planning and Scheduling, 2020.

The Section Background was replaced with a concise introduction to SLAM, and the notation adapted to be consistent with the notation introduced in this thesis. 


\subsection{Introduction}

Autonomous navigation in unknown and complex environments is an important challenge for robotics. This problem is usually tackled using path planning algorithms, such as potential field, cell decomposition, $\mathrm{A}^{*}$, or graph search [8], by relying on representations of the environments: the maps. These maps are usually built using Simultaneous Localisation and Mapping, or SLAM algorithms [9]. However, in many interesting applications, a complete map of the environment is expensive to obtain or difficult to keep up-to-date.

To avoid the need of the map, several map-less Reinforcement Learning-based path planners have been proposed in the literature for solving target-reaching ${ }^{1}$ navigation tasks in unknown environments with static or simple dynamic obstacles configurations [84], [128], [129], [130] and [131]. Even though very successful, these planners do not exploit much of the important information stored in the maps.

On the other hand, a few approaches tried to combine the learning-based planners with maps and SLAM. In this category, we find the work in [132], [133] and [134]. Here, the agent can observe the 2D-map of the environment, the robot's heading direction, the previously estimated pose and the actions taken to learn the optimal policy. However, while observing the map helps the agent choosing the best action, these methods suffer from the need to store the map information during training and the deployment phase on real robots. The latter may be computationally expensive and or not feasible for certain applications. Furthermore, all the approaches in this category rely on a simple discrete action space, e.g. move forward, move backward, turn left and turn right, that only allows coarse movements.

Here, we present a Reinforcement Learning-based method for addressing the problem of mobile robot navigation that tries to combined the best of the mapless and map-based Reinforcement Learning planners worlds by relying on maps only during the training phase for improving the performances of the planner, but not during the testing and deployment phase. To do that, we shape the reward function used to train the agent, using some of the information stored in the map of the training environment. In [16] and [17], we improved the collision-awareness of the Reinforcement Learning-based path planner by shaping the reward function

\footnotetext{
${ }^{1}$ The goal of the Reinforcement Learning agent in this context is to control and steer the mobile robot to different target locations without colliding.
} 
with respect to the position of the obstacles in the map. However, better collision avoidance is not enough when the environments grow in complexity and present local minima. For example, when the robot is in a particular room, and the target is on the other side of the wall. Therefore, in this chapter, we present and discuss a new reward function, including the map's entropy. This addition allows enhancing the exploration skills of the planner and its ability to escape local minima of navigation that are common in complex and realistic environments. The proposed framework is shown in Figure 8.1.

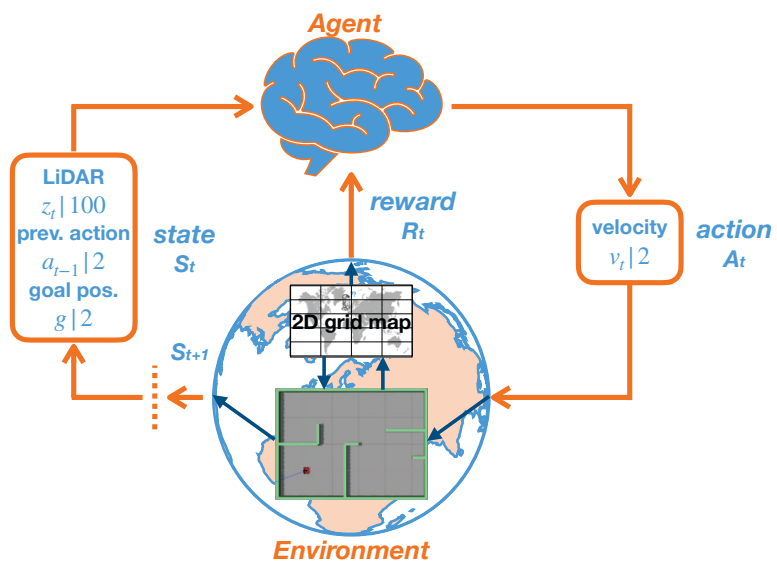

Figure 8.1: Proposed architecture for mobile robot navigation in unknown environment combining Reinforcement Learning and SLAM.

\subsection{Concise Introduction to Simultaneous Local- isation and Mapping for Robotics}

Simultaneous Localisation and Mapping, or SLAM, [9] is the given name for the mathematical methods aiming at simultaneously building the map of the environment and estimating the pose ${ }^{2}$ of the given sensor ${ }^{3}$ within it. Because of the uncertainties in the motion and the sensor readings, the estimation of the map and the pose translates into estimating a joint posterior distribution. In the case

\footnotetext{
${ }^{2}$ The pose includes position and orientation.

${ }^{3} \mathrm{We}$ assume the sensor, used for observing the environment, is carried by a mobile robot, therefore, the problem of estimating the sensor's pose is equivalent to the problem of estimating the robot's pose.
} 
of the full SLAM problem, in Equation (8.1), the joint posterior probability of the map and the complete trajectory, conditioned to the history of readings and control actions, is estimated:

$$
p\left(\chi_{0: t}, m \mid o_{0: t}, a_{0: t-1}\right)
$$

where $\chi_{0: t}=\chi_{0}, \ldots, \chi_{t}$ is the sequence of robot's poses ${ }^{4}, m$ is the map, $z_{0: t}=$ $z_{0}, \ldots, z_{t}$ is the history of observations coming from the sensor and $a_{0: t-1}=$ $a_{0}, \ldots, a_{t-1}$ the history of control actions estimated through the odometry readings of the robot. The joint posterior, in Equation (8.1), is often expensive to compute or even untrackable. In all the situations in which the computational efficiency is more important than the accuracy of the estimates, for example, in embedded applications with limited computational power, it is possible to solve, instead, the online SLAM problem, where the posterior of the map and the current pose is estimated, as shown in Equation (8.2).

$$
p\left(\chi_{t}, m_{t} \mid z_{0: t}, a_{0: t-1}\right)
$$

where only the current pose $\chi_{t}$ is considered in the computations.

Even though many SLAM solutions have been proposed for decades, such as, for example, the extended Kalman filter, or EKF, SLAM [135], the FastSLAM [136], or the ORB-SLAM [137] algorithm, it is still an open and challenging problem due to complexity of the estimation of the posterior distribution, especially in the case of the full SLAM problem, which generally needed to be computed recursively, accurately and in real-time. For more information on SLAM and probabilistic robotics, we refer the reader to [9], while for a complete overview on the most recent SLAM algorithm to [138].

\subsubsection{Rao-Blackwellized Particle Filter SLAM}

In our studies, we employ the Rao-Blackwellized particle filter, or RBPF, SLAM algorithm [139] for solving the estimation problem of the joint posterior in Equation (8.1). The RBPF approach [140] separates the estimation of the joint distribution into the problem of estimation of the posterior of the robot's trajectory and the

\footnotetext{
${ }^{4}$ The robot's pose $\chi$ is often referred in literature as state.
} 
problem of estimation of the posterior of the map, as shown in Equation (8.3).

$$
p\left(\chi_{0: t}, m \mid z_{0: t}, a_{0: t-1}\right)=p\left(m \mid x_{0: t}, z_{0: t}\right) p\left(\chi_{0: t} \mid z_{0: t}, a_{0: t-1}\right)
$$

where again $\chi_{0: t}$ represents the robot's trajectory, $z_{0: t}$ the set of observations, $a_{0: t-1}$ the control inputs and $m$ defines the map of the environment. The factorisation allows to make the estimation of the joint posterior of map and trajectory trackable by splitting the problem in two sub-problems easier to solve:

- Pose estimation with particle filter.

- Mapping with known poses.

\section{Pose Estimation with Particle Filter}

The posterior distribution of the robot's pose $p\left(\chi_{0: t} \mid z_{0: t}, a_{0: t-1}\right)$ is estimated by applying a particle filter. Particle filters estimate the belief of the robot's pose with a finite set of $N$ particles:

$$
\mathcal{X}=\left\{\chi^{(1)}, \ldots, \chi^{(2)}, \ldots, \chi^{(N)}\right\}
$$

where each particle $\chi^{(n)}$ represents the belief of what the robot's pose may be at time step $t$.

At each iteration of the particle filter algorithm, first, a new set of particles $\mathcal{X}_{t}$, with $t$ the iteration number, is computed from the previous set of particles $\mathcal{X}_{t-1}$ by sampling from a proposal distribution $X$, then a weighting factor $w$ is assigned to the particles in relation to their likelihood, as shown in Equation (8.5).

$$
w_{t}^{(n)}=\frac{p\left(\chi_{0: t}^{(n)} \mid z_{0: t}, a_{0: t-1}\right)}{X\left(\chi_{0: t}^{(n)} \mid z_{0: t}, a_{0: t-1}\right)}
$$

A standard proposal distribution is the probabilistic motion model $p\left(\chi_{t} \mid \chi_{t-1}, a_{t-1}\right)$ 
resulting into the following importance weighting update:

$$
\begin{aligned}
w_{t}^{(n)} & =\frac{p\left(\chi_{0: t}^{(n)} \mid z_{0: t}, a_{0: t_{1}}\right)}{p\left(\chi_{t}^{(n)} \mid \chi_{t-1}^{(n)}, a_{t-1}\right)} \\
& =\frac{\eta p\left(z_{t} \mid \chi_{0: t}^{(n)}, z_{0: t-1}\right) p\left(\chi_{t} \mid \chi_{t-1}, a_{t-1}\right)}{p\left(\chi_{t}^{(n)} \mid \chi_{t-1}^{(n)}, a_{t-1}\right)} \cdot \frac{p\left(\chi_{0: t-1}^{(n)} \mid z_{0: t-1}, a_{0: t-2}\right)}{p\left(\chi_{t-1}^{(n)} \mid \chi_{t-2}^{(n)}, a_{t-2}\right)} \\
& \propto \frac{p\left(z_{t} \mid m_{t-1}^{(n)}, \chi_{t}^{(n)}\right) p\left(\chi_{t}^{(n)} \mid \chi_{t-1}^{(n)}, a_{t-1}\right)}{p\left(\chi_{t}^{(n)} \mid \chi_{t-1}^{(n)}, a_{t-1}\right)} \cdot w_{t-1}^{(n)} \\
& =p\left(z_{t} \mid m_{t-1}^{(n)}, \chi_{t}^{(n)}\right) \cdot w_{t-1}^{(n)}
\end{aligned}
$$

where $w_{t}^{(n)}$ is the weight of the particle $n$ at the iteration $t$ of the algorithm, $w_{t-1}^{(n)}$ is the weight of the particle $n$ at iteration $t-1, \eta=1 / p\left(z_{t} \mid z_{0: t-1}, a_{1: t-1}\right)$ is a normalisation factor from the Bayes' rule and $m_{t-1}^{(n)}$ is the map estimation at the iteration $t-1$ of the algorithm given the particle $\chi_{t-1}^{(n)}$. In the case of the RBPF, a map is built for each particle. In the chosen RBPF approach [139], a different proposal distribution than the motion model is used to improve the accuracy of the resulting estimations. The proposal distribution also includes the most recent observation, and it is shown in Equation (8.7).

$$
p\left(\chi_{t} \mid m_{t-1}^{(n)}, \chi_{t-1}^{(n)}, z_{t}, u_{t-1}\right)=\frac{p\left(z_{t} \mid m_{t-1}^{(n)}, \chi_{t}^{(n)}\right) p\left(\chi_{t}^{(n)} \mid \chi_{t-1}^{(n)}, a_{t-1}\right)}{p\left(z_{t} \mid m_{t-1}^{(n)}, \chi_{t-1}^{(n)}, u_{t-1}\right)}
$$

Analogously to Equation (8.6), using the proposal distribution in Equation (8.7), the importance weight of a particle is proportional to:

$$
w_{t}^{(n)} \propto p\left(z_{t} \mid m_{t-1}^{(n)}, \chi_{t-1}^{(n)}, a_{t-1}\right) \cdot w_{t-1}^{(n)}
$$

After assigning the importance weight to all the particles, we need to resample them based on their importance weights ${ }^{5}$. To reduce the risk of not resampling good particles and reduce the number of times this step is performed, a resampling threshold $N_{\text {eff }}$, determining when the resampling should take place, is introduced [139]:

$$
N_{\text {eff }}=1 / \sum_{n=0}^{N}\left(\tilde{w}^{(n)}\right)^{2}
$$

\footnotetext{
${ }^{5}$ The higher the importance weight of a particle, the higher is its chance to be resampled. Importance resampling is the probabilistic version of Darwin's survival of the fittest theory [9].
} 
where $\tilde{w}^{(n)}$ is the normalised weight of the particle $n$ When $N_{\text {eff }}<N$ the resampling is performed.

\section{Mapping with Known Poses}

While it is clear to humans what a map is, how a computer represents maps may not be straightforward. In general, a map is the list of objects present in the environment and their poses. Two possible types of maps are commonly used by SLAM algorithm: feature-based and location-based maps. Featured-based maps store the locations of the features, or landmarks, in the environment. Features can be, for example, the corner of the rooms or specific textures or objects. A feature-based representation is used, for example, by EKF SLAM. Location-based maps, instead, explicitly model each possible location of the world. Therefore they include the feature locations and properties of the space, such as the occupancy of such position. A commonly used location-based map representation is the so-called occupancy grid map, in which a grid discretises the world, and each cell of the grid is updated to be occupied if an object is present at such location, unoccupied if the location is free, or unknown if no measurements have been recorded there yet. Occupancy grid maps are often used in mobile robot navigation due to the easiness of computing collision-free paths for the robots given this kind of representation.

The RBPF SLAM algorithm we utilize employs an occupancy grid map for representing the world. After the estimation of the pose, we can eventually estimate the posterior of the map $p\left(m \mid \chi_{0: t}, z_{0: t}\right)$. This step is usually called mapping with known poses [141], as we assume that the trajectory estimated by the particle filter $\chi_{0: t}$ is assumed to be known and ideally corresponds to the true trajectory followed by the robot. By making this assumption, the posterior probability of the map $p\left(m \mid z_{0: t}, \chi_{0: t}\right)$ can be computed as the product of the posterior of each individual cell $m_{i}$ of the grid, This factorisation is shown in Equation (8.10).

$$
p\left(m \mid z_{0: t}, \chi_{0: t}\right)=\prod_{i=0}^{M-1} p\left(m_{i} \mid z_{0: t}, \chi_{0: t}\right)
$$

where $M$ is the total number of cells in the map. Occupancy grid map can be easily built using LiDAR readings, as it easy to derive the occupancy properties of the space withing the range of the sensor. For more information, we, again, refer the reader to $[9]$. 


\subsection{Methodology}

\subsubsection{Proposed Approach}

We propose a Reinforcement Learning-based path planning algorithm that exploits the information stored in the map of the training environment to improve the training speed, the generalisation and the robustness of the policy learned, but that does not require the map at testing time for achieving higher computational efficiency.

The Reinforcement Learning agent uses only raw sensory information $z_{t}$, i.e. LiDAR readings, the previously chosen action $a_{t-1}$ and the goal position $g$, for determining the sequence of continuous velocity commands, linear and angular velocities respectively, that the low-level robot's controllers have to track for steering the robot and reaching the target locations in environments with unknown obstacle configuration. Differently from standard map-less Reinforcement Learning-based motion planners, only during the training phase, we exploit the knowledge of the environment stored in the map, built online using the RBPF SLAM algorithm, to improve the training speed and navigation skills of the agent. The incorporation of the knowledge stored in the map happens through the shaping of the reward function. Moreover, unlike approaches that employ only target-driven reward functions (see Equation (8.11)) dependent on the distance to the target with the addition of penalties for hitting obstacles, to aid the navigation in complex environments, we proposed a new reward function including the distance to the obstacles and the map's entropy to encourage exploratory behaviours and, hopefully, escape local minima.

\subsubsection{Reward Function}

The agent's goal is control and steer a mobile robot through high-level velocity commands. The robot must be able to reach the desired targets while avoiding collisions with obstacles and escape local minima. To achieve these three objectives, we propose a reward function equal to the weighted sum of three different terms:

- Target-driven (TD) [84].

- Obstacle-awareness (OA) [131]. 
- Map's entropy (Entropy).

The TD term of the reward function, in Equation (8.11), is based on the Euclidean distance from the robot' current position to the target. In particular, this term is proportional to the difference of the distance from the target at the previous time step $\left\|\chi_{t-1}^{(x, y)}-g\right\|_{2}$ and the distance from the target at the current time step $\left\|\chi_{t}^{(x, y)}-g\right\|_{2}$. Intuitively, the agent is positively rewarded if it gets closer to the target and negatively if it gets farther away.

$$
r_{1}\left(s_{t}, a_{t}\right)=\left\|\chi_{t-1}^{(x, y)}-g\right\|_{2}-\left\|\chi_{t}^{(x, y)}-g\right\|_{2}
$$

where $\left\|\chi_{t}^{(x, y)}-g\right\|_{2}$ is the distance, or $L_{2}$-norm, from the current position $\chi_{t}$ of the robot at time $t$ with respect to the inertial frame and the target's location $g$ expressed in the robot's coordinate frame.

The OA term of the reward function, in Equation (8.12), includes a penalty inversely proportional to the squared distance to the obstacles. In this way, the agent is negatively rewarded if it gets closer to obstacles. It is worth to mention that the distance to the obstacle is computed online using the LiDAR readings and the position of the obstacles is not assumed to be known a priori. This term of the reward function is activated once the distance to an obstacle, measured with the LiDAR, is smaller or equal than $0.6 \mathrm{~m}$.

$$
r_{2}\left(s_{t}, a_{t}\right)=\frac{1}{d_{t}^{2}}
$$

where $d_{t}$ is the minimum distance measured given the array of LiDAR readings at a give time instant $t$.

The third term of the reward function includes the map's entropy and it is shown in Equation (8.13). The map's entropy corresponds to the sum of the entropy of all the cell $c$ in the map $m$, therefore it is a measure of the uncertainties we have on the map's estimate. The agent is positively rewarded if it reduces the uncertainties in the map, therefore if it explores the environment by navigating through open and unknown areas.

$$
r_{3}\left(s_{t}, a_{t}, m\right)=\sum_{c_{f} \in m} p(c) \log p(c)+\sum_{c_{o} \in m}(1-p(c)) \log (1-p(c))
$$

where $c_{f}$ corresponds to the unknown and unoccupied cells in the map and $c_{o}$ to 
the occupied ones.

In addition, a sparse reward, $r_{\text {reached }}$, is added if the agent reaches the target within a predefined distance threshold and a penalty, $r_{\text {crashed }}$, if the robot either collides with an obstacle or exceeds the maximum number of steps $T$ in a single episode without reaching any other terminal condition. Based on the above considerations, the overall reward function $\mathrm{R}(s, a, m)$ in shown in Equation (8.14).

$$
\mathrm{R}(s, a, m)= \begin{cases}r_{\text {reached }}, & d \leq d_{\text {min }} \\ r_{\text {crashed }}, & s=s_{t s}, \\ \lambda^{T D} r_{1}(s, a)-\lambda^{O A} r_{2}(s, a)-\lambda^{H} r_{3}(s, a, m), & \text { otherwise. }\end{cases}
$$

where $\lambda^{T D}, \lambda^{O A}$ and $\lambda^{H}$ are scalar weighting factors for the three reward terms $r_{1}(s, a), r_{2}(s, a)$ and $r_{3}(s, a, m)$ used to balance their contributions.

\subsubsection{Reinforcement Learning Algorithm}

Because we would like to generate smooth navigation trajectories, we employ a continuous action space. Therefore, we choose the DDPG algorithm (see Section 3.2.2) as the candidate Reinforcement Learning algorithm for determining the velocity commands, linear and angular velocities, of the robot to navigate to different target locations without colliding with obstacles or getting stuck in local minima. Again, it is worth mentioning that the Reinforcement Learning algorithm represents the high-level controller of the robot. Once the agent's actions are determined, the robot's low-level controllers execute each action by sending the appropriate torque commands to each actuator.

The state vector $s_{t}$ is composed by 100 LiDAR data-points $z_{t}$ uniformly distributed on a $360^{\circ}$ range, the action chosen at the previous time step $a_{t-1}$ and the current distance from the target position expressed in Euclidean coordinates $g$. The state vector is 104-dimensional and it is shown in Equation (8.15).

$$
s_{t}=\left[z_{t}, a_{t-1}, g\right]
$$

It is worth mentioning that by not including the map $m$, or the complete sequence of LiDAR readings $z_{0: t}$ and robot's poses $x_{0: t}$, in the state vector, we partially violate the MDP modelling framework in which the reward function $\mathrm{R}$ is a function 
of the state of the environment. However, locally, the current LiDAR readings $z_{t}$ are a good approximation of the map of the environment at time step $t$. Therefore, because the agent has to select actions within the LiDAR readings range, we can still hold the MDP framework without any reduction of performances.

\section{Neural Network Architectures}

The neural network architecture consists of mainly three parts: feature extraction, policy, or actor, and critic networks, as shown in Figure 8.2. The feature extrac-

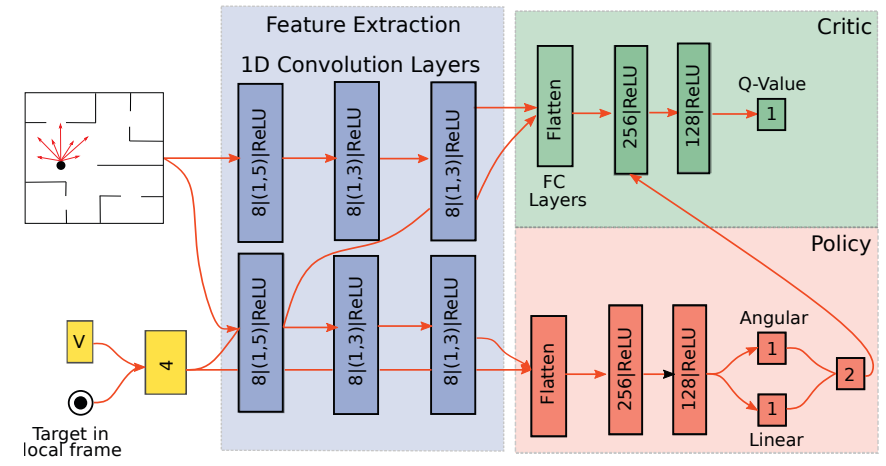

Figure 8.2: The laser readings are pre-processed by the feature extraction block consisting of three convolution layers. Only in the first fully-connected layer of the network, the target information and the previous velocity commands are fused with the LiDAR features.

tion block of the network is responsible for extracting features from the LiDAR readings through three $1 \mathrm{D}$-convolutional layers. Each $1 \mathrm{D}$-convolutional layer has eight filters, stride length two, ReLU activations and kernel sizes of five, three and three, respectively. The convolutional layers are used to reduce the number of parameters of the networks and achieve better generalisation in unseen environments. After the feature extraction block, we find the policy network. The policy network comprises three fully-connected layers, of size 256-128-2 respectively, with input the features extracted from the LiDAR reading concatenated with the previously chosen velocity commands and the target position, and output the linear and angular velocities for the robot low-level controllers.

The linear velocity range is constrained in $[0,1]$ to allow only forward motion, while for the angular velocity, the range is constrained in $[-1,1]$ to allow right and left rotation. Thus, sigmoid and tanh activation functions are used respectively in 
the output layer, while the remaining layers employ ReLUs. Eventually, the critic network, with architecture analogous to the actor, estimates the Q-values of the state and action pairs using three fully-connected layers, respectively, with ReLu activations and with linear activation at the output. The first fully-connected layer of the critic additionally concatenates the current action $a_{t}$ for the sake of the estimation of the $\mathrm{Q}\left(s_{t}, a_{t}\right)$.

\subsection{Experimental Design}

\subsubsection{Simulation Setup}

The virtual 3D environment is built using the Robot Operating System, or ROS, middleware and Gazebo simulator. The experiments are conducted on an Ubuntu 16.04 machine with an Intel Core i7-8550 CPU. The algorithms are written using the OpenAI-ROS package provided by the ROS. The simulated robot is the skidsteering Husarion. The robot is controlled through velocity commands that are directly sent to the low-level controllers, with update frequency equal to $10 \mathrm{~Hz}$. The agent control loop waits until the velocity set-points are tracked with an error smaller than a given threshold. The feedback for the low-lever controllers is provided by estimating the robot's velocity from the encoder's readings, i.e. the odometry. Furthermore, the robot is equipped with $360^{\circ} 2 \mathrm{D}$-LiDAR for sensing the environment.

\subsubsection{Configuration of the RL and SLAM algorithms}

For training the Reinforcement Learning policy and value function, we use the ADAM optimiser [142]. The hyperparameters are chosen based on the ones used in the original paper of the DDPG [143] and are shown in Table 8.1. The exploration noise is chosen as an Ornstein-Uhlenbeck (OU) process with parameters $\sigma=0.2$ and $\theta=0.15$, since these values have empirically shown good performances. Furthermore, L2-regularization is included when training the critic network to prevent overfitting and improve generalisation.

For simultaneously mapping the environment and estimating the robot pose, we use the RBPF SLAM building an occupancy grid map, the gmapping SLAM package is used [144]. A probability value is assigned to each cell based on whether 


\begin{tabular}{||c|c||}
\hline Parameter & Value \\
\hline \hline optimiser & ADAM \\
actor learning rate & $10^{-3}$ \\
critic learning rate & $10^{-4}$ \\
L2-regularization coefficient & $10^{-2}$ \\
discount factor $\gamma$ & 0.99 \\
target networks update $\tau$ & 0.001 \\
OU-noise $\sigma$ & 0.2 \\
OU-noise $\theta$ & 0.15 \\
batch size & 64 \\
\hline
\end{tabular}

Table 8.1: DDPG hyperparameters for the experiments.

it is occupied, free or unknown accordingly to the LiDAR and odometry readings. Similar to [145], the occupancy threshold value is chosen equal to 0.65 , and if the probability value of the cell is greater than this threshold, this cell is occupied or, conversely, free otherwise. Based on the probability assigned to every grid cell, the entropy of the map is calculated according to Equation (8.13). The complete list of parameters' values used for the SLAM algorithm can be found in Table 8.2.

\begin{tabular}{||c|c||}
\hline Parameter & Value \\
\hline \hline occupancy threshold & 0.65 \\
map update threshold & 1.0 \\
grid cell size & $5 \mathrm{~cm} \mathrm{x} 5 \mathrm{~cm}$ \\
LiDAR max. range & $3 \mathrm{~m}$ \\
\hline
\end{tabular}

Table 8.2: RBPF hyperparameters for the experiments.

\subsubsection{Training and Testing}

To validate the effectiveness of the approach, we compare the performance of the path planner trained using the proposed reward function, in Equation (8.14), with the performances of the path planner trained used only the TD reward term ${ }^{6}$, as in [84], and one trained with the TD, and OA reward terms ${ }^{7}$, as in [131].

For a fair comparison, the same hyperparameters are used as well as the same

\footnotetext{
${ }^{6}$ This is equivalent to set the coefficients of the obstacle-awareness term $\lambda^{O A}$ and the entropy coefficient $\lambda^{H}$ to zero in Equation (8.14).

${ }^{7}$ Again this is equivalent to set the entropy term $\lambda^{H}$ to zero in Equation (8.14).
} 
neural network architectures. First, the three different Reinforcement Learning

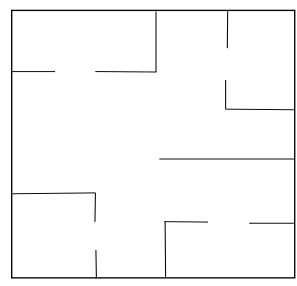

(a) Env-1

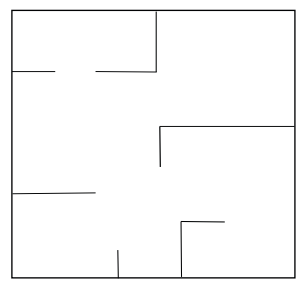

(b) Env-2

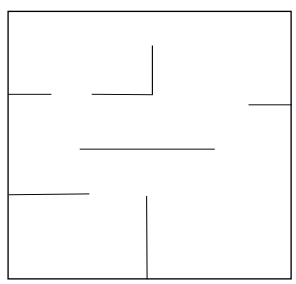

(c) Env-3

Figure 8.3: The agents are trained in Env-1. Then, the performances of the policies are evaluated in the unseen a priori environments Env-2 and Env-3. All the environments are of size $4.5 \mathrm{~m} \times 4 \mathrm{~m}$.

agents are trained in Env-1, shown in Figure 8.3a, and then tested in environment $E n v-1$ on the same set of 100 randomly generated targets. For each training episode, the target location $g$ and the initial pose of the robot $p_{0}$ are sampled from a uniform distribution to avoid biasing the policy toward a specific target, starting location, and environment topology and improve the generalisation skills of the planners. For the path planner trained using the reward function in Equation (8.14), the map is also reset at the start of a new episode.

Furthermore, to assess the generalisation properties of the policies, we evaluate the planners in a priori unseen environments: Env-2 and Env-3, in Figure 8.3b and $8.3 \mathrm{c}$ respectively, again on the same set of 100 randomly generated targets.

Eventually, we compare the proposed learning-based agent with move_base, the dynamic window approach, or DWA, a path planner [146] of the navigation stack in ROS. Differently from the proposed approach, move_base requires the complete map of the environment to be built beforehand to plan paths. Eventually, it is worth mentioning that move_base requires the tuning of several parameters to achieve good performance.

\subsubsection{Real World Experiments}

Obtaining real data from robotic systems can be extremely difficult, costly and time-consuming. For example, model-free Reinforcement Learning algorithms usually require a considerable number of training samples before convergence ${ }^{8}$. However, simulation with accurate models could potentially be used to reduce, or even

\footnotetext{
${ }^{8}$ We need more than $10^{5}$ samples in the navigation problems under study
} 
to cancel, the cost of real-world interactions. For the real-world experiments, a Clearpath Jackal differential drive mobile robot, Figure 8.4, is used as the mobile robot platform. After training the agent in the simulated platform ${ }^{9}$ in Env-4 in

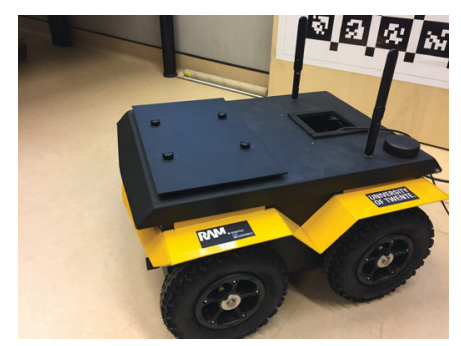

Figure 8.4: Clearpath Jackal mobile robot used in the real-world experiments.

Figure 8.5, the learned policy is transferred directly to the real robot in an environment with similar topology without any extra tuning of the policy parameters or further training using real-world samples. To evaluate the quality of the trans-

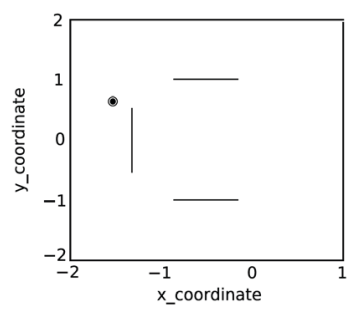

Figure 8.5: Env-4 is environment used for the transfer learning experiments.

ferred policy, different target positions are randomly chosen for the robot, and a comparison is made between the travelled trajectory on the real and virtual environments. Eventually, we evaluate the approach in environments with untrained topology.

\footnotetext{
${ }^{9}$ It is worth mentioning that for these experiments, the Clearpath Jackal simulation model is used instead of the Husarion one.
} 


\subsection{Results and Discussion}

\subsubsection{Training and Testing}

\section{Training Results}

To compare the training performances of the different agents, we study the collision ratio in relation to the training episodes. The results are shown in Figure 8.6. The

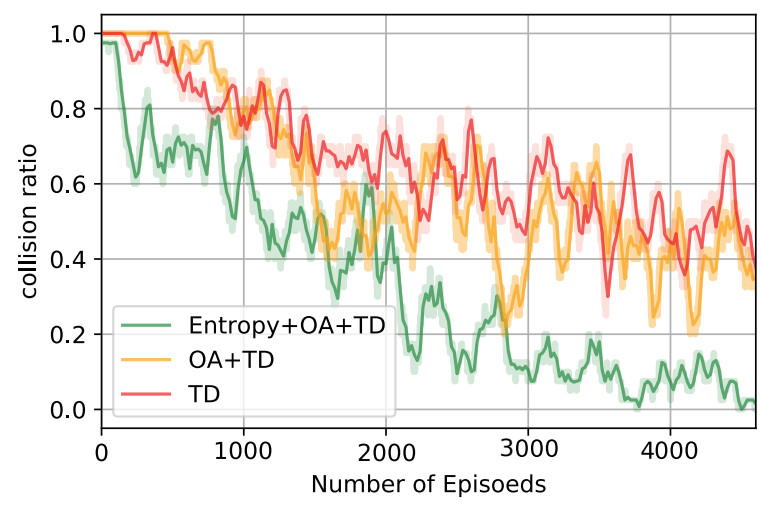

Figure 8.6: Evolution of the collision ratio with the number of training episodes. The solid line represents the mean value and the shade its variance. The amount of collisions of the proposed approach Entropy+OA+TD decreases much faster than the TD only and TD+OA reward functions.

agent trained only with the TD reward function (red line in Figure 8.6) struggles to reduce the number of collisions during training, as it receives penalties only when the collision has already occurred. Learning to navigate safely and away from the obstacle is more challenging when only a sparse reward signal is used. When the environment is fairly complex, the agent trained with TD and OA reward function (yellow line in Figure 8.6), even though slightly superior, also struggles to improve the policy. However, this is due to the environment topology and the local minima, and it is discussed in detail in the following Section. Eventually, the agent trained with the proposed reward function (see Equation (8.14)) achieves the best training performances by reaching a collision ratio constantly smaller than 0.2 in only 3000 training episodes. This is because the entropy term pushes the agent to explore the environment better and escape local minima more easily. Furthermore, throughout the training, the agent trained with the proposed approach is the only one able 
to reduce the fluctuation in the collision ratio consistently. These fluctuations are due to the random spawning of the robot and the target location at the beginning of each episode. This is an additional sign of higher generalisation and robustness of the proposed planner.

\section{Testing Results}

After training the three agents in Env-1, the learned policies are evaluated on the same set of 100 randomly generated targets in each of the three environments. To guarantee a fair comparison, the percentage of the successes crashes, and timeouts $^{10}$ is recorded and presented in Figure 8.7.

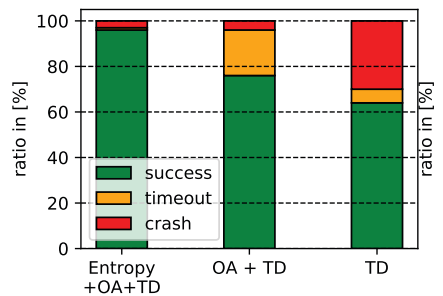

(a) Env-1.

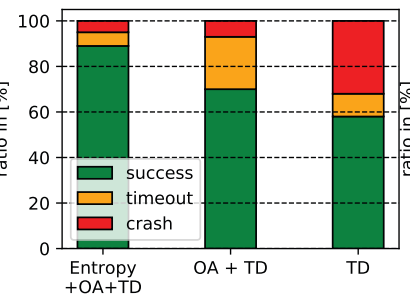

(b) Env-2.

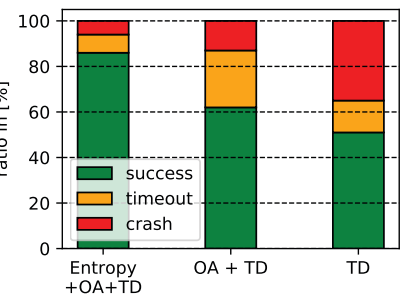

(c) Env-3.

Figure 8.7: Performance comparison in the different environments of the three planners.

In the training environment Env-1, the planner trained with TD can reach only $64 \%$ of the targets, and, in most of the failed episodes, it collides with an obstacle, while the planner trained with TD and OA achieves better performances. This planner can reach $76 \%$ of the targets and reduce the crashes compared to TD only, from $32 \%$ to $3 \%$. However, the enhanced obstacle-awareness, in the case of complex environments with local minima, prevents the agent from colliding, but it is not enough to prevent the robot from getting stuck in local minima until the maximum number of actions is reached. This aspect can be noticed in the increment of the timeouts with respect to TD only, from $4 \%$ to $21 \%$. On the other hand, the planner trained with the reward function in Equation (8.14) is the only one that can achieve good performances and reaches the target in the $96 \%$ of the episodes.

\footnotetext{
${ }^{10}$ We define timeout an episode ending with the maximum number of actions taken without either reaching the target or colliding.
} 
The crucial aspect for any learning-based approach is the generalisation to untrained situations, as we can not train and gather data for each possible scenario. To assess the generalisation skills of the agents, we transfer the policies learned in Env-1 to the unseen environments Env-2 and Env-3. Consistently with the training results, the proposed approach outperforms the other two in terms of success ratio, respectively $89 \%$ against $68 \%$ and $58 \%$ in Env-2 and $84 \%$ against $62 \%$ and $51 \%$ in Env-3, reduction of the collisions, $3 \%$ against $6 \%$ and $33 \%$ in Env-2 and $7 \%$ against $13 \%$ and $36 \%$ in Env-3, and timeouts, $8 \%$ against $26 \%$ and $8 \%$ in Env-2 and $9 \%$ against $25 \%$ and $13 \%$ in Env-3.

For all three environments, we also record and analyse the average number of steps per episode and the standard deviation as essential elements for assessing the length and smoothness of the trajectories of the different agents. Table 8.3 presents these results and recaps what discussed in the previous paragraph. The

\begin{tabular}{||c|c|c|c||}
\hline & approach & $\begin{array}{c}\text { success ratio } \\
\%\end{array}$ & $\begin{array}{c}\text { number of actions } \\
(\text { mean } \pm \text { std })\end{array}$ \\
\hline \hline Env-1 & TD & $64 \%$ & $113.07 \pm 85.43$ \\
& OA+TD & $76 \%$ & $84.56 \pm 76.31$ \\
& Entropy+OA+TD & $\mathbf{9 6} \%$ & $\mathbf{5 9 . 2 8} \pm \mathbf{3 7 . 9 9}$ \\
\hline Env-2 & TD & $58 \%$ & $140.68 \pm 99.17$ \\
& OA+TD & $68 \%$ & $131.34 \pm 86.78$ \\
& Entropy+OA+TD & $\mathbf{8 9} \%$ & $\mathbf{1 2 4 . 0 7} \pm \mathbf{7 4 . 8 6}$ \\
\hline Env-3 & TD & $51 \%$ & $115.55 \pm 84.38$ \\
& OA+TD & $62 \%$ & $76.32 \pm 67.48$ \\
& Entropy+OA+TD & $\mathbf{8 4} \%$ & $\mathbf{5 7 . 1 4} \pm \mathbf{4 0 . 3 9}$ \\
\hline
\end{tabular}

Table 8.3: Performance assessment of the three planners in Env-1, Env-2 and Env-3.

agent trained with the TD reward term learns overcautious behaviours and travels away from the obstacles. This penalises the trajectories length, and it can be noticed from the high average number of steps. When trained with TD and OA reward terms, the agent can reduce the cautiousness and learn shorter paths in terms of the average number of steps. However, when local minima are present, as in Env-2, the planner often gets stuck in them, and its performances are not much better than TD. Instead, the planner trained with the proposed reward function can learn shorter trajectories with respect to the other two thanks to the enhanced exploration granted by the entropy term. Even in unseen environments, 
the agent can escape local minima by effectively incorporating into the navigation skills the exploration ones. The complete video of the experiments can be found at: https://youtu.be/KxRPBX8npis.

Eventually, we compare the trajectories generated by the proposed path planner and the one generated by move_base on the same set of targets (see Figure 8.8) that has to be reached in sequence in Env-1. Both planners can successfully reach all targets. However, the total travelled distance required to complete the sequential task by the proposed planner is shorter, $14.7 \mathrm{~m}$ compared to $16.3 \mathrm{~m}$, than the one required by the move_base. In addition, the path generated by move_base appears to be less smooth than the one generated by the proposed planner.

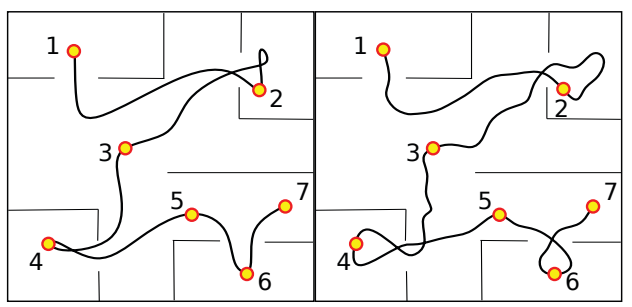

(a) proposed planner

(b) move_base

Figure 8.8: A comparison between the proposed map-less motion planner and the DWA planner of the ROS navigation stack.

\subsubsection{Real-world Experiments}

The efficiency of transferring the learned policy has been validated on different targets, three of which are depicted in Figure 8.9. It can be noticed that the trajectories do not perfectly overlap due to the differences that always exist between the real world and simulations. Nonetheless, the robot can reach the targets without colliding with the obstacles and following reasonably good trajectories. This proves, once again, the robustness of learning algorithms against simulation mismatches and noise. Moreover, even if the real environment does not match precisely the simulated one, the policy is still robust enough to steer the robot towards the target successfully. The complete video of the experiments can be found at: https://youtu.be/qXLmpTUpHMA. 


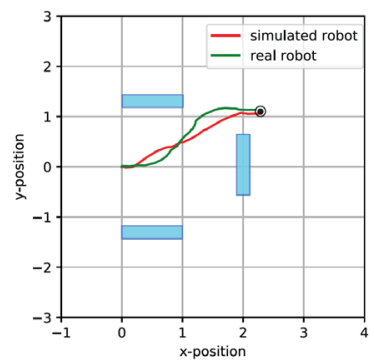

(a) Target 1

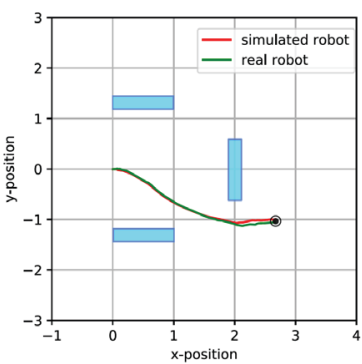

(b) Target 2

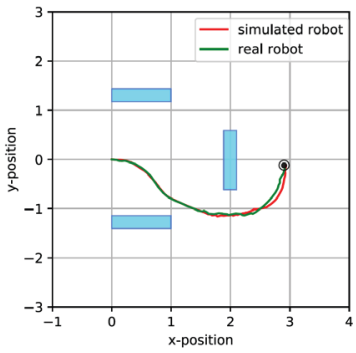

(c) Target 3

Figure 8.9: A comparison of the generated trajectories by the simulated robot in gazebo (visualized in red) and the real robot after transferring the learned policy (visualized in green). The target is depicted by the black circle.

\subsection{Conclusions}

This chapter presents a Reinforcement Learning-based path planner for navigation in unknown environments. The planner is trained by exploiting the knowledge stored in the occupancy grid map, built online during training using the RBPF SLAM algorithm. However, the planner does not rely on any map except the one used during the training phase. In particular, we use the map entropy to shape the reward function to improve the exploration skill of the agent to escape different local minima. By learning better exploration skills, compared to a target driven (TD) reward function and obstacle-aware (TD+OA) reward function, the agent trained with the proposed approach outperforms the other two not only in the training environment, the success ratio of $96 \%$ against $64 \%$ and $76 \%$ respectively, but also in two different unseen environments, the success ratio of $89 \%$ and $84 \%$ against 58\%, 51\% and 68\%, 62\% respectively. Furthermore, the agent trained with the proposed approach achieves performances comparable, if not even slightly superior, to the ones of move_base, a DWA planner that requires the map of the environment. Eventually, the policy is robust enough to be transferred to the real robot without further retraining. 


\section{Chapter 9}

\section{Curiosity-driven Reinforcement Learning Agent for Mapping Unknown Indoor}

Environments

This chapter proposes a Reinforcement Learning framework for navigating, exploring, and mapping unknown environments. The Reinforcement Learning agent is in charge of selecting the commands for steering the mobile robot, while a SLAM algorithm estimates the robot pose and maps the environments. The agent, to select optimal actions, is trained to be curious about the world. This concept translates into introducing a curiosity-driven reward function that encourages the agent to steer the mobile robot towards unknown and unseen areas of the world and the map. We test our approach in explorations challenges in different indoor environments. The agent trained with the proposed reward function outperforms the agents trained with reward functions commonly used in the literature for solving such tasks.

This chapter includes an adaptation of:

Nicolò Botteghi, Beril Sirmacek, Rob Schulte, Mannes Poel, and Christoph Brune. Reinforcement Learning helps SLAM: learning to build maps. International Society for Photogrammery and Remote Sensing Congress, 2020.

Nicolò Botteghi, Rob Schulte, Beril Sirmacek, Mannes Poel, and Christoph Brune. Curiositydriven Reinforcement Learning Agent for Mapping Unknown Indoor Environments. International Society for Photogrammery and Remote Sensing Congress, 2021.

The Section Background was removed as treated in Chapter 2 and 3, and the notation adapted to be consistent with the notation introduced in this thesis. 


\subsection{Introduction}

When the environments are unknown, efficiently constructing the maps by selecting the optimal sequence of control actions is challenging. This problem is commonly referred to in the literature as the active SLAM problem, in which not only pose and map are estimated, but also the control actions are chosen in order to explore the whole environment. The simplest solution one could think of is to rely on pre-coded navigational directives based on landmark positions and specific features, e.g. turn left when recognizing a particular landmark such as the corner of a room. However, coding these directives is problematic if the environment is not known beforehand or not static. These solutions are often brittle and struggle to adapt to environments with different topologies.

Another approach for active SLAM is the so-called frontier-based exploration [147]. This method relies on the identification of special navigational targets, i.e. the frontiers, sitting in-between the known and unknown regions of the map, selecting the most promising ${ }^{1}$ one and eventually navigate to it. Once the chosen frontier is reached, the procedure is repeated until all the frontiers are visited, and the map is complete. This approach is suitable mainly for occupancy grid-based maps, where each cell of the grid corresponds to a portion of the environment. Each cell of the grid map is either classified as free, occupied, or unknown. However, the robot's overall path to complete the map is usually not optimal in terms of distance travelled, even if the closest frontier is repeatedly selected.

As in the case of path planning, Reinforcement Learning-based methods can be used to select the control commands to steer the robot for fully exploring unknown environments such that SLAM algorithms can reconstruct their maps. However, the problem of exploratory navigation for constructing maps is more challenging than the navigation-to-a-target problem. Because there is not an explicit navigation target, the Reinforcement Learning agent needs to learn to explore. Defining a suitable non-sparse reward function is not straightforward in this case.

The problem of learning to explore, in the context of Reinforcement Learning, can be tackled by reward shaping. The goal is to reward the agent for being curious, i.e. to choose actions that allow travelling to novel or never-visited states of the environment. This is usually referred to in literature as curiosity-driven

\footnotetext{
${ }^{1}$ The most promising frontier can be chosen in different ways, such as the closest frontier, the most informative one or combinations of the two objectives.
} 
Reinforcement Learning [148].

In this chapter, we propose a Reinforcement Learning and SLAM-based framework for efficiently exploring and building maps of unknown indoor environments based on the robot's onboard sensory readings and the information coming from the SLAM algorithm, such as the pose estimate and the map's completeness. The proposed approach is shown in Figure 9.1. In particular, we introduce a curiositydriven reward function dependent on the novelty of the position visited by the robot that allows fast learning of the exploration policy and its generalisation to untrained environments. Additionally, the proposed reward function is independent of the type of map, either feature-based or location-based map, built using the SLAM algorithm.

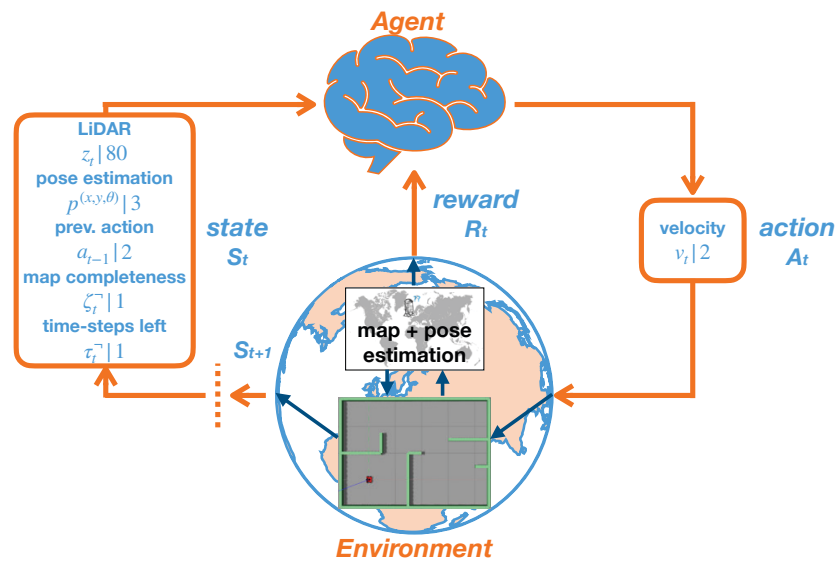

Figure 9.1: Proposed framework combining Reinforcement Learning and SLAM.

\subsection{Related Work}

Reinforcement Learning has been used in active SLAM for learning optimal exploration strategies for steering a mobile robot, carrying sensors, in unknown environments, and constructing their maps. In [149], Reinforcement Learning is used to find the most informative trajectories to reach a set of given target locations and to consequently, improve the quality of the map. However, differently from our work, we do not assume a known set of navigation targets, but we rely only on onboard sensor readings and information coming from the SLAM algorithm for steering the robot into the unknown areas of the environments. 
A Reinforcement Learning-based active SLAM architecture is proposed in [150], where an agent is trained to explore the environments in order to build their maps by relying on raw RGB-D sensor readings, a top-view image of the map, and a bump sensor for detecting collisions. The approach relies on expert trajectories to initialise the agent's policy. Differently for them, we do not rely on expert data and high-dimensional sensory reading, but only on LiDAR. Additionally, the focus of our work is to design a reward function that encourages exploration.

A similar architecture is proposed in [151], where a Reinforcement Learning agent is trained on depth images and range sensory readings for navigating without collisions and constructs maps of different environments. The reward function is shaped using multiple objectives and ultimately promotes moving forward to free space. However, this reward function has no terms related to the map coverage, therefore the agent is not necessarily rewarded for exploring quickly and/or choosing short trajectories.

In [19] and [152], the authors propose a Reinforcement Learning approach for active SLAM that relies on 2D LiDAR readings and information coming from the SLAM algorithm to select the best steering command, out of a discrete action space, for a mobile robot to explore different environments. While we use similar state space definition, we employ a continuous action space for obtaining smoother trajectories. Both approaches focus on the shape of the reward function. Differently, in our work, we propose a new curiosity-based reward function that drastically improves the generalisation skills of the agent.

Eventually, the authors of [153] propose a hybrid Reinforcement Learning and frontier-based exploration framework, where the agent is not directly choosing velocity commands nor trajectories for the robot, but simply selects the frontier to visit. An $\mathrm{A}^{*}$-based path planner is then in charge of steering the robot to the selected frontier. Despite the success, the method reduces, only slightly, the total traveled distance by the robot with respect to the original frontier-based exploration approach [154]. In our approach, we do not rely on the explicit computation of the frontier, which may be computationally expensive. Moreover, visiting all the frontiers may cause longer trajectories than necessary.

All these Reinforcement Learning-based approaches rely on random noise on the action space to explore action space and, consequently, explore the environment. We believe that is not enough for fast learning and generalisation and we employ a curiosity-based reward function. The proposed reward function may be 
beneficial to the performance of all the mentioned approaches.

\subsection{Methodology}

\subsubsection{Proposed approach}

We aim at solving the active SLAM problem using Reinforcement Learning to select the best actions to explore indoor environments and construct their maps. We assume this problem to have a finite horizon, i.e. we assume the existence of a terminal state, reachable in a finite number of steps, corresponding to the environment being fully explored and the map is fully constructed. The Reinforcement Learning algorithm chosen, i.e. DDPG (see Section 3.2.2), only relies on 80 2D-LiDAR readings, the robot's pose estimate coming from the RBPF SLAM algorithm, the previous action taken by the agent, the percentage of the map to be explored and the time steps left before the end of the episode. The time steps left and the percentage of the map left before completeness help to make the algorithm aware of the terminal conditions and consequently help the performance and stability of the learning algorithms [155]. Moreover, these two assumptions allow us to study and tackle the problem without the need for an explicit memory structure in the Reinforcement Learning algorithm. The state vector $s_{t}$ at a given time instant $t$ is shown in Equation (9.1).

$$
s_{t}=\left[z_{t}, \chi_{t}^{(x, y, \theta)}, a_{t-1}, \zeta_{t}^{\neg}, \tau_{t}^{\urcorner}\right]
$$

where $z_{t}$ corresponds to the LiDAR readings uniformly spread on a $360^{\circ}$ range, $\chi_{t}^{(x, y, \theta)}$ to the current pose estimate, i.e. $x, y$-position in the Cartesian plane and the robot's orientation $\theta, a_{t-1}$ to the previous action taken by the agent, $\zeta_{t}$ to the remaining map percentage before completeness and $\tau_{t}$ to the time steps left before the end of the episode. The agent's actions are continuous linear and angular velocity set-points that are sent and tracked by the low-level controllers on the robot.

\subsubsection{Exploration by Reward Shaping}

The exploration-exploitation trade-off is one of the biggest challenges in Reinforcement Learning. The agent has to explore the environment to find higher rewards, 
but its ultimate goal is to exploit and collect the highest possible rewards. Especially in all the situations in which a sparse reward function is used, improving the policy requires a long training time.

However, it is possible to formulate curiosity-based, or intrinsic, reward functions that motivate the agent to explore uncertain regions of the state space, e.g. states that are badly predicted, accordingly to a learned forward dynamical model, as in [148] and [128]. These approaches drastically improve the convergence of the algorithms and can solve sequential decision making processes with very sparse reward functions. When policies are learned through such reward functions, these tend to explore the whole state space indiscriminately, and they do not necessarily focus on exploring more interesting areas. This may be a limitation in vast environments. Moreover, suppose the environment is highly stochastic or random. In that case, the forward model can only poorly predict the next state, and consequently, the reward is not informative enough to allow good exploration and fast policy learning [156].

Alternatively, the novelty can be defined in terms of reachability, i.e. how many steps the agent needs to reach a specific state [76]. This approach stores a particular sub-set of novel observations and compares them with the most recent observation of the agent to determine the novelty of such observation. In their approach, the authors use a neural network to determine if the current observation can be reached within the k-steps from any of the stored observations and consequently generate a reward proportional to that distance. This method shows significant improvements in terms of convergence speed with respect to the forward model prediction and suffers less from the problem of environment stochasticity.

We propose an adaptation of the episodic curiosity reward introduced by [76] for improving the exploration skills of the Reinforcement Learning algorithm in the context of active SLAM, and we investigate its effect on the generalisation skills of the trained agent to different environment topologies. We propose a reward function that combines a bonus when the environment is fully explored and the map is completed, a penalty when collisions occur and an intrinsic reward promoting exploration of unknown locations. The propose reward function, named 
Curiosity, is shown in Equation (9.2).

$$
\mathrm{R}(s, a)= \begin{cases}r_{\text {map completed }}, & \text { if } \zeta_{t} \geq C \\ r_{\text {crashed }}, & \text { if } z_{t} \leq z_{\text {min }} \\ r_{t}^{c}, & \text { otherwise }\end{cases}
$$

where $r_{\text {map completed }}$ is the bonus given to the agent when the percentage of map completeness $\zeta_{t}$ is above a threshold ${ }^{2} C, r_{\text {crashed }}$ is a negative penalty for getting too close, accordingly to a fixed threshold $z_{\text {min }}$, to obstacles according the current LiDAR reading $z_{t}$ and $r_{t}^{c}$ is the curiosity term to promote exploration.

To compute the curiosity-based reward, we first define a finite-size buffer $\mathcal{E}$ with cardinality $E$ containing novel robot positions $\chi_{t}^{(x, y)}$, estimated via the SLAM algorithm. A position is novel if it is at least at a distance $k$ from all the other positions visited, with $k$ a parameter of our algorithm. If a novel position is found during exploration, this is added to the novelty buffer. A graphical illustration of the curiosity reward is shown in Figure 9.2.

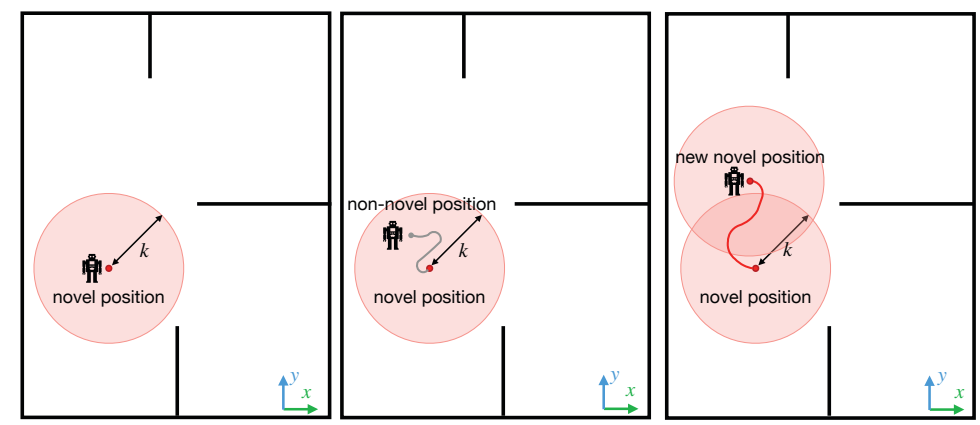

Figure 9.2: Intuitive representation of the novelty circle with radius $k$.

At each time step of the algorithm, the curiosity reward term is computed by averaging the sum of the Euclidean distance between the current robot position estimate $\chi_{t}^{(x, y)}$ and all the novel position $\chi_{1 \ldots E}^{(x, y)}=\chi_{1}^{(x, y)}, \ldots, \chi_{E}^{(x, y)}$ in the novelty

\footnotetext{
${ }^{2}$ Due to small mapping errors, the map is rarely completed exactly at $100 \%$. Therefore, we set the map-completeness threshold to $93 \%$ to prevent never reaching such a condition.
} 
buffer, as shown in Equation (9.3).

$$
r_{t}^{c}=\frac{\alpha}{E} \sum_{i=1}^{E} d\left(\chi_{t}^{(x, y)}, \chi_{i}^{(x, y)}\right)
$$

where $\alpha$ is a constant scaling the novelty reward term and controlling the urgency in reaching novel positions, $M$ is the number of elements of the novelty buffer and $d$ is the Euclidean distance operator. If $r_{t}^{c}$ is higher than a certain threshold for a given position $\chi_{t}^{(x, y)}$, then this is added to the novelty buffer.

If the buffer is complete and a new novel position is found, an old element is randomly discarded. In this way, anything that is easily reachable by the agent becomes quickly uninteresting since no further reward can be obtained from it, while reaching positions further away from the known positions, i.e. at least distant $k$, is encouraged. This reward drives the robot to unexplored areas by taking advantage of the pose estimate from the SLAM algorithm. Moreover, because only the pose estimate is used, the proposed approach is independent of the map the SLAM algorithm is constructing by making the approach suitable for different scenarios.

Positions close to the walls are not considered novel positions since this would encourage undesired behaviours. A benefit of this is that the agent gains a natural tendency to select actions keeping the robot in the middle of the rooms. The choice of $k$ is intuitively crucial for the fast learning of the Reinforcement Learning algorithm. If $k$ is chosen too small, any position can, in principle, become novel and be appended to the novelty buffer. This choice would slow down the exploration and consequently reduce convergence speed. On the other side, if $k$ is picked too large, the curiosity reward term tends to disappear because novel positions may be too difficult to reach as too distant.

The complete algorithm used for computing the curiosity reward is presented in Algorithm 10, where the outer loop iterates over the number of episodes and the inner loop over the number steps allowed in an episode ${ }^{3}$.

\footnotetext{
${ }^{3}$ These two loops are the standard ones for any episodic Reinforcement Learning algorithm.
} 


\section{Pseudo-code 9.1: Episodic Curiosity Reward}

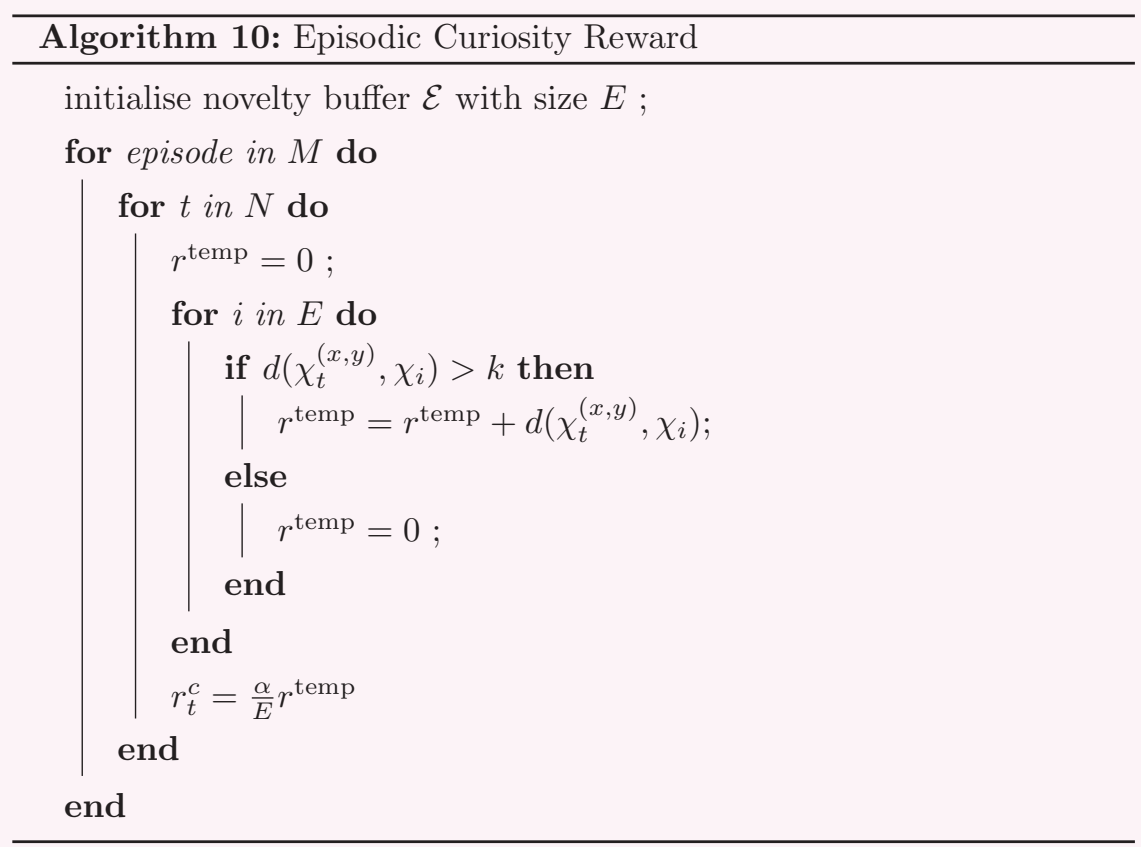

\subsubsection{Neural networks architecture}

In the DDPG algorithm, both the policy and value function are approximated by neural networks. In particular, the state vector is fed to the actor network, which is composed of three fully-connected hidden layers with ReLU activations and 512 neurons each. Eventually, the output layer has dimensionality 2 and outputs the linear and angular velocities for the robot. To constrain the velocities to feasible values for the robot's actuators, we use a sigmoid and a tanh activation for the linear and angular velocity, respectively. In this way, we constrain the robot to move only forward, but we leave the possibility to turn left or right.

The critic network is also composed of the three fully-connected hidden layers with ReLU activations and 512 neurons each. However, while the state vector is fed to the first hidden layer of the network, the action is only fed into the second layer. The output layer has linear activation and a single output, i.e. the estimated Q-value of the input state-action pair. 


\subsection{Experimental Design}

\subsubsection{Setup}

The experiments are performed using the Robot Operating System (ROS) and the simulation platform Gazebo. Gazebo allows simulating robots, their dynamics, realistic environments and sensors. In contrast, ROS allows communication among all the elements of the simulation platform, e.g. robot and sensors, and the learning algorithm. Additionally, ROS allows easy integration with different SLAM packages and the gmapping package that is used in this research.

The learning algorithm is written in Python using the Tensorflow library for the neural networks construction and training and OpenAI-gym for the Reinforcement Learning environment.

\subsubsection{Baselines}

We compare the proposed reward function, in Equation (9.3), with three different reward functions used in literature and in Equation (9.4)-(9.6). In particular, we focus on the learning speed of the agents trained with different reward functions, the length and quality of the trajectory after training, the percentage of map completed and the generalisation to untrained environments.

We first compare with a sparse reward function, named Sparse:

$$
\mathrm{R}(s, a)= \begin{cases}r_{\text {map completed }}, & \text { if } \zeta_{t} \geq C \\ r_{\text {crashed }}, & \text { if } l_{t} \leq l_{\text {min }} \\ 0, & \text { otherwise }\end{cases}
$$

Then, we compare with the reward function based on the map completeness gain, named Oracle, similar to the one adopted by [150]:

$$
\mathrm{R}(s, a)= \begin{cases}r_{\text {map completed }}, & \text { if } \zeta_{t} \geq C \\ r_{\text {crashed }}, & \text { if } l_{t} \leq l_{\text {min }} \\ \zeta_{t}-\zeta_{t-1}, & \text { otherwise }\end{cases}
$$

Moreover, we compare with a reward function based on the entropy, named 
Information-gain, similar to the one used in [19]:

$$
\mathrm{R}(s, a)= \begin{cases}r_{\text {map completed }}, & \text { if } \zeta_{t} \geq C \\ r_{\text {crashed }}, & \text { if } l_{t} \leq l_{\text {min }} \\ H_{t}-H_{t-1}, & \text { otherwise. }\end{cases}
$$

where $H_{t}$ is the map's entropy at time $t$ and $H_{t-1}$ is the map's entropy at time $t-1$.

Eventually, all the learning-based approaches are compared with the frontierbased exploration [147].

\subsubsection{Training and Testing}

We are interested in studying the effect of the reward function on the performance and generalisation skills of the agents. To do that, we train the agents in a single environment, Env-1, and evaluate their performances on two unknown ones. The training environments is the Env-1, shown in Figure 9.3a, while the testing environments, Env-2 and Env-3, are shown in Figure $9.3 \mathrm{~b}$ and $9.3 \mathrm{c}$ respectively.

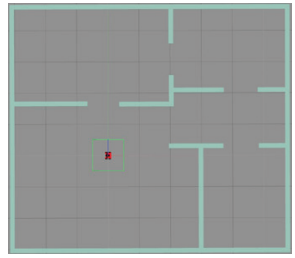

(a) Env-1

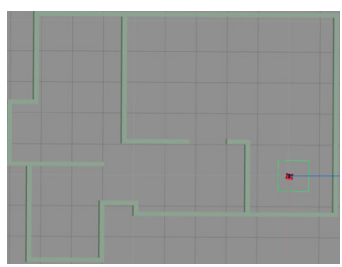

(b) Env-2

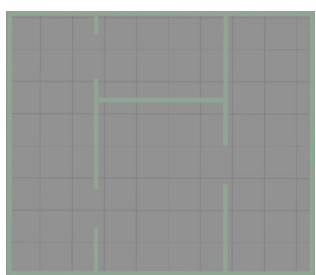

(c) Env-3

Figure 9.3: The training environment, Env-1, and testing environments, Env-2 and Env-3 used for training and evaluating the proposed approach.

The training environment Env-1, is based on a real $65 \mathrm{~m}^{2}$ apartment from the Dutch housing website funda [157]. Mapping the whole environment provides a significant challenge since a random policy is not enough for exploring the whole space and construct its map efficiently. The second and the third environments, Env-2 and Env-3, have total areas of $68.5 \mathrm{~m}^{2}$ and $75 \mathrm{~m}^{2}$ and they are, again, based on real apartments from [157]. These two environments are only used for testing the generalisation capabilities of the trained agents, and no policy-learning is performed here. 
The proposed approach is independent of the chosen mapping algorithm. However, in our experiments, we employ the RBPF SLAM algorithm [139] with occupancy grid-based map, which is discussed in Chapter 8, Section 8.2.1.

We train each agent for the same amount of episodes on environment Env1 by employing two different training strategies. Firstly, the robot position, at the beginning of each episode, is kept the same for the whole training process ${ }^{4}$. Secondly, at the beginning of each episode, the robot is randomly spawned in one of four possible starting positions. The parameters used in our experiments are shown in Table 9.1.

\begin{tabular}{||c|c||}
\hline RL and SLAM parameters & Value \\
\hline \hline optimiser & ADAM \\
actor learning rate & $10^{-3}$ \\
critic learning rate & $10^{-4}$ \\
discount factor $\gamma$ & 0.99 \\
batch size & 64 \\
replay buffer size & $10^{6}$ \\
novelty threshold $k$ & $1.8 \mathrm{~m}$ \\
grid cell size & $5 \mathrm{~cm} \times 5 \mathrm{~cm}$ \\
occupancy threshold & 0.60 \\
LiDAR max. range & $10 \mathrm{~m}$ \\
LiDAR min. range & $0.2 \mathrm{~m}$ \\
\hline
\end{tabular}

Table 9.1: Parameters of the experiments.

After training, each agent is tested in the unseen a priori environment Env2 and Env-3, and the map completeness and the trajectory length and quality are recorded. For each testing environment, four different starting positions are selected, and three experiments per position are performed.

\subsection{Results and Discussions}

\subsubsection{Training Results}

The map-completeness ${ }^{5}$, and the numbers of actions per episode of the different agents during training are presented in Figure 9.4a and 9.4b. When only a single

\footnotetext{
${ }^{4}$ The chosen starting position is a point in the middle of the bottom-left room of environment Env-1.

${ }^{5}$ Because we are employing different reward functions, the reward values obtained by the agents are different. Therefore, we compare the training performances based on how fast the map is completed.
} 
fixed starting position for the robot is chosen in each training episode, the agent trained with the curiosity-driven reward function outperforms the other in terms of map-completeness rate, by quickly converging after $\sim 150$ episodes, and numbers of actions compared to the other agents. The proposed curiosity-based reward function quickly motivates the agent to choose actions that can steer the robot to unseen locations of the environment, as far as possible from the known ones.

On the other side, when the robot is spawned at different starting locations, the agent trained with the information-gain reward function, in Equation (9.6) and the one trained with the map-completeness reward function, in Equation (9.5), achieve performance comparable to the curiosity, if not even slightly superior, to the proposed curiosity-based reward function in terms of map-completeness over training. These two methods benefit the most from the random initialisation of the robot's position. However, when we analyse the number of actions taken in each training episode, the proposed reward function reduces the actions taken at a higher rate than the other reward functions. In this context, the sparse reward function, in Equation (9.4), without a memory structure, e.g. a recurrent policy network, does not converge to a good solution in the time span of 400 episodes.

\subsubsection{Generalisation Results}

We test how well the planners trained in environment Env-1 perform in the unseen a priori environment Env-2 and Env-3. Additionally, we compare the learningbased planners with frontier-based exploration. In Table 9.2, the generalisation results in environment Env-2 and the ones related to environment Env-3 are shown, where we compare the planners by recording the average map-completeness and trajectory length in twelve different runs for each planner with four possible starting positions. The trajectories for all the runs are shown in Figure 9.5 and Figure 9.6 for the testing in environment Env-2 and Env-3 respectively.

Compared to the other agents, the one trained with the proposed reward function achieves the highest map-completeness in both environments and travels smoother and shorter paths than the others. It is worth mentioning that when trained with the Sparse and Oracle reward functions, the agent never and rarely, respectively, completes the maps, and they show a circling behaviour on the spot with low velocity. Because we fix the maximum number of actions in an episode to 500 , the distance travelled by such planners is low. 


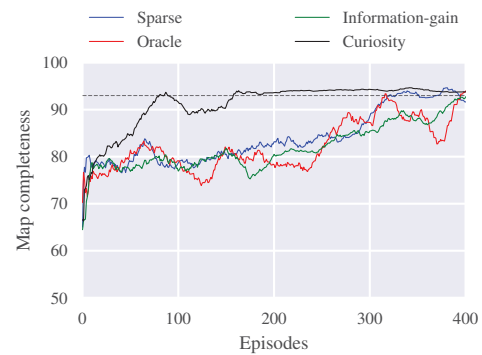

(a)

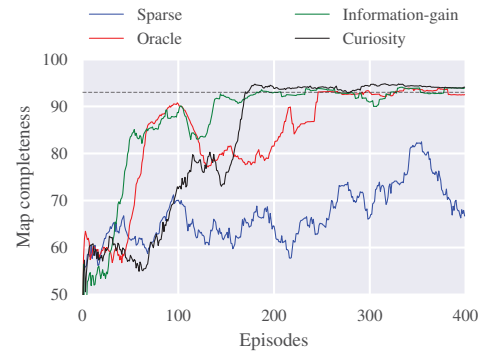

(c)

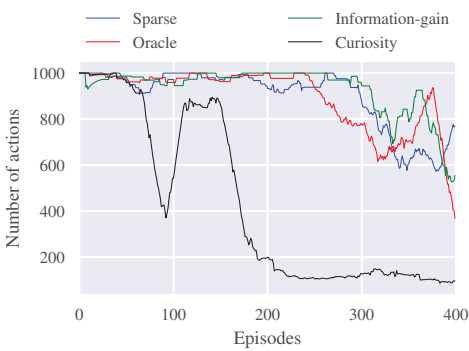

(b)

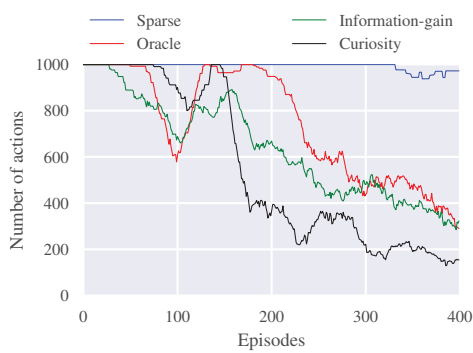

(d)

Figure 9.4: The training results in Env-1 when the robot starts each training episode in the same position, Figure $9.4 \mathrm{a}$ and $9.4 \mathrm{~b}$, and when its pose is randomly selected, Figure 9.4c and 9.4d. The Sparse corresponds to the sparse reward function, the Oracle to the map-completeness reward function, the Informationgain to the entropy based reward function and Curiosity to the proposed reward function.

\begin{tabular}{||c|c|c|c||}
\hline & approach & $\begin{array}{c}\text { map-completeness \% } \\
\text { (mean } \pm \text { std) }\end{array}$ & $\begin{array}{c}\text { traj.length }(\mathrm{m}) \\
\text { (mean } \pm \text { std) }\end{array}$ \\
\hline \hline Env-2 & Sparse & $55.86 \pm 17.44$ & $11.86 \pm 3.35$ \\
& Oracle & $65.28 \pm 18.53$ & $15.89 \pm 4.4$ \\
& Information-gain & $93.48 \pm 8.94$ & $24.31 \pm 5.87$ \\
& Curiosity & $99.09 \pm 0.65$ & $17.65 \pm 5.14$ \\
& Frontier & $98.45 \pm 0.366$ & $15.68 \pm 3.6$ \\
\hline Env-3 & Sparse & $37.08 \pm 7.88$ & $10.7 \pm 2.25$ \\
& Oracle & $66.83 \pm 11.29$ & $18.87 \pm 10.33$ \\
& Information-gain & $88.03 \pm 6.54$ & $18.78 \pm 4.04$ \\
& Curiosity & $92.58 \pm 6.69$ & $25.23 \pm 7.93$ \\
& Frontier & $99.15 \pm 0.29$ & $25.38 \pm 4.08$ \\
\hline
\end{tabular}

Table 9.2: generalisation results in the untrained environment Env-2 and Env-3.

The curiosity-driven agent achieves performances comparable to the frontier based explorations in terms of average map completeness and trajectory length. 


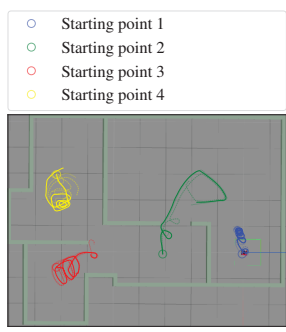

(a) Sparse

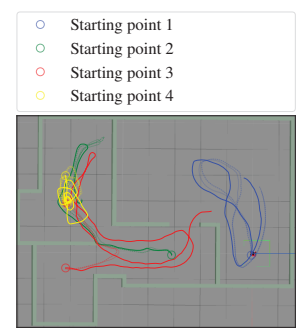

(b) Oracle

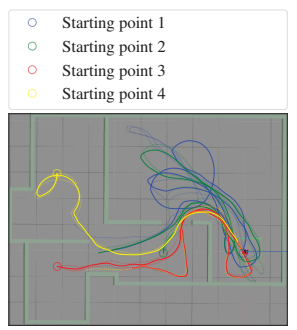

(c) Information-gain

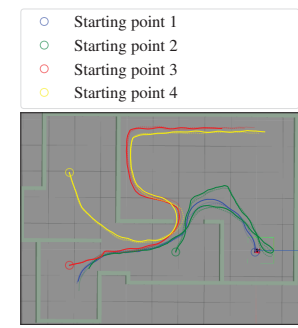

(d) Curiosity

Figure 9.5: Evaluation of the trajectories of the agents trained with the different reward functions in the environment Env-2.

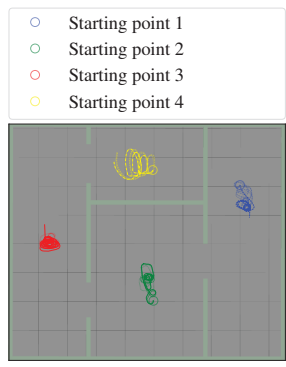

(a) Sparse

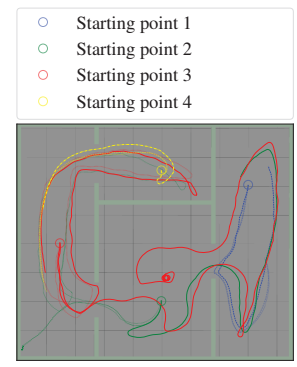

(b) Oracle

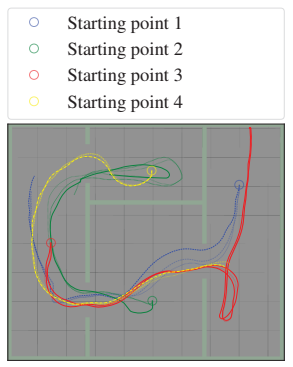

(c) Information-gain

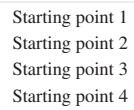

Starting point 2

Starting point 3

Starting point 4

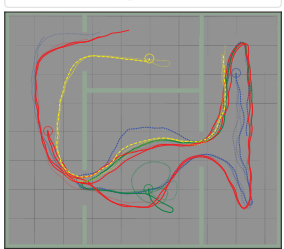

(d) Curiosity

Figure 9.6: Evaluation of the trajectories of the agents trained with the different reward functions in the environment Env-3.

However, it is worth mentioning that the proposed planner has higher computational efficiency than the frontier, where more operations have to be performed at each time step, such as detecting the frontiers, choosing one accordingly to a predetermined criterium, and navigating to the frontiers without collisions. A short video of our experiments can be found at: https://youtu.be/QPIEpUeg9h0.

\subsection{Conclusions}

The key element of the success of the approach is to transform the problem of exploration of unknown environments into the problem of visiting novel locations of the world and the map. This result is achieved by shaping the reward function used to train the Reinforcement Learning agent to encourage its curiosity into unseen locations and, consequently, features. The Reinforcement Learning agent, 
trained with the proposed curiosity-driven reward function, outperforms in terms of generalisation to untrained environments, map-completeness, and trajectory length and smoothness, the agents trained with commonly used reward functions for such tasks. The proposed approach achieves performance comparable to the frontier-based exploration method but with a lower computation cost. Moreover, the approach is not limited to occupancy-grid maps but can cope with any map representation and SLAM algorithm. This is because the algorithm for training and testing only requires the robot's pose estimate and the completeness of the map. 


\section{Conclusions and Discussions}

\section{IV}

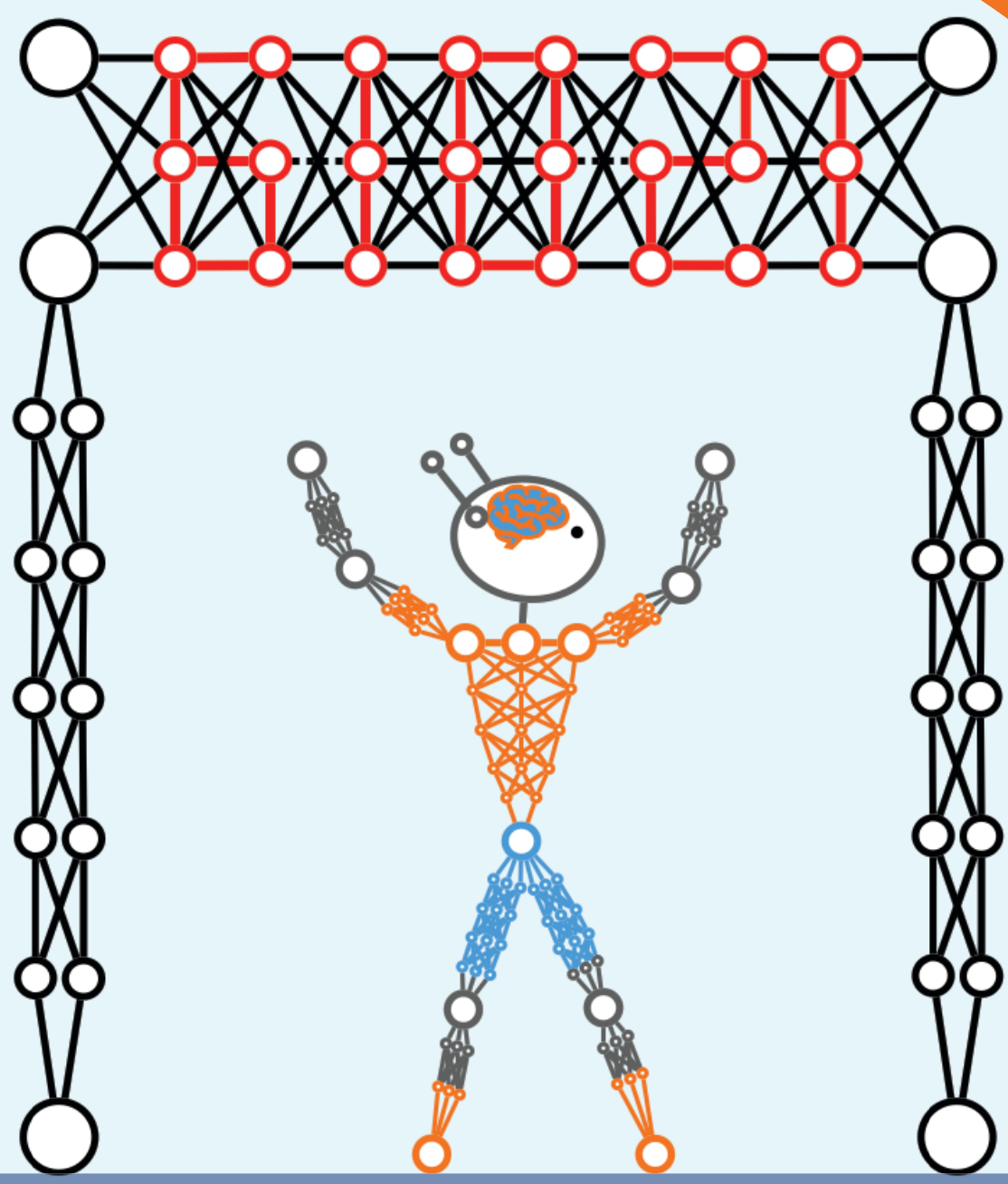





\section{Chapter 10}

\section{Conclusions}

Not all those who wander are lost.

J.R.R. Tolkien - The Fellowship of the Ring

In this thesis, we have broadly studied robotics Deep Reinforcement Learning with different forms of prior knowledge.

We have efficiently and effectively combined the power of Reinforcement Learning, Deep Learning and prior knowledge. We have proposed several approaches for dealing with the different aspects of Reinforcement Learning, such as state representation learning, the joint learning of state and action representation, the hierarchical decomposition of the actions, and reward shaping.

We have chosen the general yet crucial autonomous mobile robot navigation and exploration problems as the application domain. Here, the Reinforcement Learning agent acts as the high-level controller that drives the mobile robot through unknown environments by only relying on its on board sensory readings. Because learning optimal policies directly on the real robot is infeasible due to the fragility of the hardware and the long training times required, we have used virtual simulators using approximated mathematical models of the world. Therefore, we have sought optimal solutions that can bridge the simulation-to-reality gap. 


\subsection{The Answers}

Firstly, we have studied approaches for learning meaningful representations of the unknown state space of the environment. In this thesis, we chose the road of unsupervised learning methods, i.e. without the need for labelled data, which makes the problem more challenging but more general and applicable, as labelled data may not always be available or easy to obtain. However, we incorporate prior knowledge into the state representation learning step. This challenge has led to the following question and its consequent answer:

$\diamond$ How can one learn state representations by incorporating prior knowledge of the environment?

By introducing prior knowledge into the loss functions, through the shaping of their kernels, using knowledge of the world (e.g. physics), reward properties, and underlying structures of continuous action spaces, to guide the learning of the meaningful state features and regularise the learned state space $^{1}$.

Secondly, we have studied the problem of jointly learning state and action representations using the MDP homomorphism metrics to guarantee that the optimal solution of the learned abstract MDP is the optimal solution of the original MDP. This research has led to the following question and its answer:

$\diamond$ How can one jointly learn state and action representations by integrating prior knowledge of state and action space?

By assuming an underlying low-dimensional structure of state and action spaces, it is possible to transform a high-dimensional MDP into a lowdimensional MDP by learning the mappings from observation space to latent state space and from action space to latent action space with neural networks in a self-supervised fashion. The optimal solution of the abstract MDP is sample efficient to find and more robust to disturbances, such as unseen beforehand features, and optimal for the original $\mathrm{MDP}^{2}$.

Thirdly, we have studied the problem of learning action abstractions under the umbrella of Hierarchical Reinforcement Learning by defining the policy hierarchy

\footnotetext{
${ }^{1}$ The support to this answer is presented in Chapter 4 and 5.

${ }^{2}$ The support to this answer is presented in Chapter 6.
} 
using prior knowledge and leaving enough freedom to learn generic and generalisable policies given the fixed hierarchy. We study this problem for the complex snake-like pipe inspection robot PIRATE. These studies have led to the following question and its answer:

$\diamond$ How can one learn optimal policies by exploiting prior knowledge of the problem structures?

By decomposing the policies using our knowledge of the problem, we can exploit problem structures, and by learning from scratches the policies, we can improve generalisation, flexibility, and learning efficiency of the overall behaviour ${ }^{3}$.

Finally, we have studied the problem of reward shaping. Shaping reward functions is one of the most studied problems in Reinforcement Learning as crucial means for learning efficiency. Here, we have studied this problem in combination with SLAM algorithms for navigation and exploration. We have shaped the reward functions using the knowledge stored in the maps of the environments. For autonomous navigation purposes, we have shaped the reward function using information about obstacles in the map to reduce collisions and the map's entropy to reduce uncertainties and escape navigation minima. For autonomous exploration, instead, we shape the reward function based on the novelty of the position visited by the robot to obtain a curious agent capable of generalising its exploratory behaviour to untrained environments.

$\diamond$ How can one learn optimal policies by shaping the reward function using prior knowledge of the maps of the environments?

By incorporating map information such as the position of the obstacles, entropy, and novelty of the robot's pose in the reward function, we can improve performances, training efficiency, generalisation and robustness of the learned policy ${ }^{4}$.

By studying all these different aspects of Reinforcement Learning, namely, state, observation, and action spaces and reward function, we can answer the main research question of this thesis:

\footnotetext{
${ }^{3}$ The support to this answer is presented in Chapter 7.

${ }^{4}$ The support to this answer is presented in Chapter 8 and 9.
} 
What is "good" prior knowledge for robotics Reinforcement Learning for a) improving generalisation, b) sample efficiency, and c) robustness of the learned behaviours?

Good knowledge for robotics Reinforcement Learning is the prior knowledge of the environment or the task that guides the agent to learn better representations and policies without constraining its learning. We refer to this prior knowledge as loose prior knowledge. This concept concretely translates into:

$\Rightarrow$ Shaping loss functions based on properties of the underlying state space (e.g. physical knowledge) when learning state representations (Chapter 4 and 5).

$\Rightarrow$ Enforcing low-dimensionality of latent state and action spaces (Chapter 6).

$\Rightarrow$ Exploiting actions hierarchies when learning complex policies (Chapter 7).

$\Rightarrow$ Shaping reward functions based on known properties of the environment and the task (Chapter 8 and 9).

\subsubsection{The Implications}

In Part I, we discussed the general Reinforcement Learning framework [1] (see Figure 10.1a), widely applicable to sequential decision making process. Throughout these studies, we have found out that such a scheme for end-to-end learning can be further improved to increase sample efficiency, generalisation, exploitation of problem structures, and accommodation of prior knowledge ${ }^{5}$. Therefore, we proposed an adaptation of the classical Reinforcement Learning scheme proposed in [1] to account for all these elements. This scheme is shown in Figure 10.1b.

Learned representations ${ }^{6}$ are the key elements of this framework. The scheme combines sample-efficient learning of a low-dimensional state representation ${ }^{7}$, using a neural network $\phi$, with an action abstraction ${ }^{9}$, learned using a neural network $\delta$, and with reward shaping, either through prior knowledge or coming from the

\footnotetext{
${ }^{5}$ The latter is crucial for robotics Reinforcement Learning.

${ }^{6}$ Such as the representation learned by the observation encoder $\phi$ and the action decoder $\delta$ in Figure 10.1b.

${ }^{7}$ Throughout this thesis, we have focused on neural networks for modelling policies, value functions ${ }^{8}$, state and action representations, but in principle, any other method for function approximation could be used.

${ }^{9}$ Any action abstraction approach is suitable for the framework (either learned or set by prior knowledge such as a hierarchical decomposition).
} 


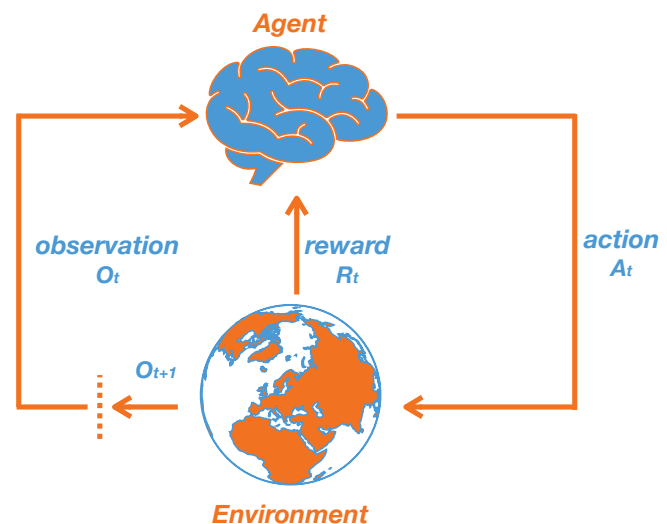

(a) Classical Reinforcement Learning framework.

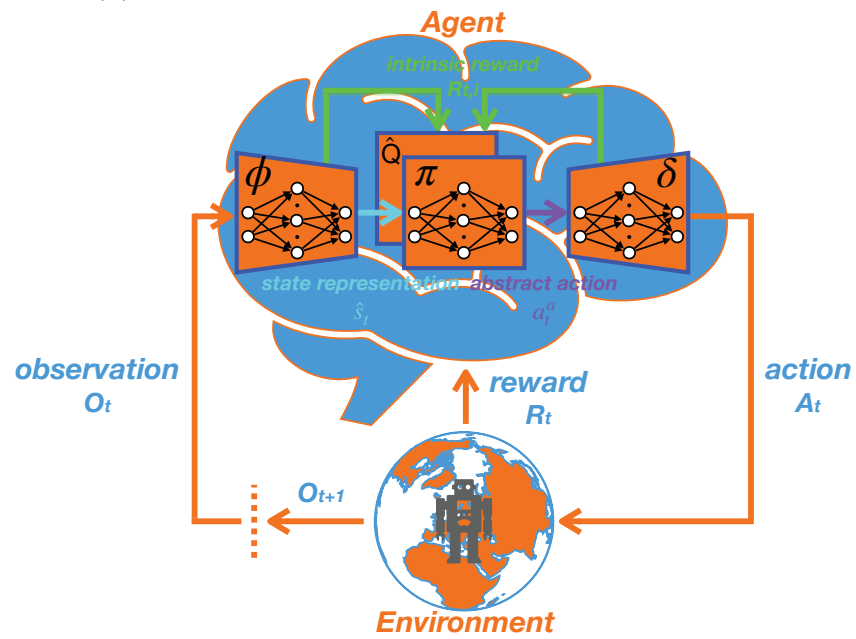

(b) Proposed robotics Deep Reinforcement Learning framework.

Figure 10.1: Robotics Reinforcement learning.

learned representation ${ }^{10}$. This framework can potentially solve all the curses of robotics Reinforcement Learning presented in Chapter 3, Section 3.4.

\footnotetext{
${ }^{10}$ The learned representations can be used to define curiosity-driven, intrinsic reward functions that can improve the exploration and the learning efficiency of the algorithms.
} 



\section{Chapter 11}

\section{Discussions}

Good research finds new answers, great research finds new questions. 


\subsection{Robotics Deep Reinforcement Learning}

In our journey towards autonomous robots, we have chosen the road of learning behaviours by interacting with the world using the Reinforcement Learning paradigm. This learning approach does not usually require prior knowledge to find optimal solutions but requires huge amounts of data (and patience). While sample efficiency may not be a limiting factor in some contexts, this is a severe drawback of robotics in which robots need to interact with the real world. The cost of real-world samples is usually too high ${ }^{1}$ and the interaction is simply time inefficient $^{2}$. To overcome this issue, we have chosen to rely on virtual simulators and physical engines that depend on mathematical models of the world. Simulators allow safe and efficient learning ${ }^{3}$. However, it is not straightforward to transfer what learned in the simulation to the real world due to the so-called simulation-toreality gap. This problem gets more severe and evident whenever the task grows in complexity and uncertainties, for example, when the agent uses high-dimensional observations, e.g. camera images, to learn the optimal policy. Visual features are challenging to model in the simulated environment, e.g. due to shadows and light reflections. Therefore, the policies learned on those features are difficult to transfer to the real world.

In the context of robotics Deep Reinforcement Learning, one could then think of (over)constraining the problems by adding and heavily relying on prior knowledge. However, while prior knowledge is always available for simple tasks, it is not realistic to think we can always know and pre-code optimal solutions beforehand. Moreover, these constraints limit the power of Deep Reinforcement Learning and function approximators, such as neural networks. Neural networks can represent any function, and Deep Reinforcement Learning algorithms can learn any policy in principle. However, there are no guarantees that the optimal solution or representation can be found at all. It is clear that for sample efficient regimes, such as the ones sought in robotics Reinforcement Learning, the prior knowledge, guiding the learner, plays an important role.

Learning representations of the data is the key element for the success of Deep Learning algorithms. When such a concept is brought into the Reinforcement

\footnotetext{
${ }^{1}$ Robots can break and wear, and they are usually composed of expensive hardware.

${ }^{2}$ Real-world experiments cannot be sped up and countless hours are needed.

${ }^{3}$ Simulators replace real-world interaction and can simulated reality several times faster than real-time.
} 
Learning world, we usually speak of state, or action, representation learning. Instead of directly learning a policy from high-dimensional observations, it is possible first to learn an explicit (low-dimensional) representation of the (input) data and consequently feed such a representation to the Reinforcement Learning agent to learn the optimal policy. Good representations, when learned, allow great robustness, generalisation and transferability of the learned policies. Representations are crucial for reducing the simulation-to-reality gap introduced by the use of virtual simulators. Again, any representation of the input data can be learned through a neural network. However, not every representation is meaningful for the agent. This problem is even more acute in unsupervised learning settings, where it is not straightforward to learn meaningful representations without any form of supervision. In the context of unsupervised state representation learning, prior knowledge can be used to guide the learning of the representation by enforcing the regularisation of the learned space. Another advantage of using (low-dimensional) representations is that the problems suddenly become more interpretable and understandable.

\subsection{The New Questions}

Based on our discoveries, many new questions have arisen for the future. In this section, we try to concisely summarise the most relevant ones for the future of Robotics Reinforcement Learning:

- Generalisation to unseen situations and meta-learning $\rightarrow$ we cannot simply keep adding data to our dataset.

- Efficient exploration $\rightarrow$ we cannot solve challenging problems with random exploration.

- Unifying model-free and model-based Reinforcement Learning $\rightarrow$ we need extreme sample efficiency for solving complex problems.

- Scaling to real-world problems $\rightarrow$ we need to close the gap between current Reinforcement Learning applications and real-world applications. 


\subsubsection{Generalisation to Unseen Situations and Meta-learning}

The meta-learning paradigm, often referred to as the learning to learn paradigm, focuses on the learning of new skills for quick adaptation and better generalisation to different environments with limited, or no, extra data. In the context of meta-Reinforcement Learning, this typically translates into the learning of policies that can generalise across different tasks and environments without complete retraining from scratches. While policy generalisation is of crucial importance for Reinforcement Learning agents, meta-learning ideas could and should be brought to all the other elements of the agent (see Figure 10.1), namely the state and action representations, but also, in principle, rewards.

For example, in the context of state representation learning, we showed, in Chapter 4 and Chapter 5, that the agent can efficiently learn a representation for a single environment, in which each wall has a different colour, and consequently the optimal policy using such a representation. If now, after the training of the representation and policy in a specific environment, we would change the colours, or the position, or the shape of the wall, the agent is prone to fail, even when the optimal policy is the same. Analogous examples can be found for action representations and policies decomposition. It is clear that for further progressing, learning not only general-purposes policies but also generic and adaptable state and action representations is crucial to retain sample efficiency and robustness, and to further improve generalisation across tasks and environments. Therefore, we have formulated a new research question to tackle such a challenge:

What is the role of meta-learning for the problem of state and action representations learning in Reinforcement Learning?

\section{Learned Priors}

In Chapter 4 and 5, we introduced the problem of unsupervised state representation learning using the robotics priors. The priors are means to incorporate prior physical knowledge into the loss functions for learning state representations. However, priors may not always be available, nor easy to hand-craft in the form of loss functions ${ }^{4}$, nor the same across different tasks or environments. In order to seek generalisation, it is interesting to investigate the possibility of learning such

\footnotetext{
${ }^{4}$ Loss functions based on complex physical laws may have a complex structure and many coefficients to tune.
} 
loss functions using first choosing their parametrisation and then learning such parameters, given the data from the environments. These learned priors can then be used to learn the state representation. This interesting research direction leads to:

To what extend one can learn the priors of the world into the form of loss functions, and consequently learn generalisable state representations based on the learned priors?

\subsubsection{Efficient Exploration}

Exploring better and more efficiently is still one of the big open problems of Deep Reinforcement Learning and one of the major limiting factors for solving complex problems. The method for collecting samples influences not only the learning of policies and value functions but also the learning of the state and action representations by creating biases in the data. In the latter case, the assumption of having a set of randomly collected samples is often employed. This assumption is, however, very restrictive in many complex scenarios.

Intrinsic reward functions, promoting an agent's curiosity towards the unknown and exploratory behaviours, play an important role in efficient Reinforcement Learning. All these approaches use low-dimensional representations of the observation to identify what is unpredictable or unseen beforehand ${ }^{5}$. However, it is not clear what their contribution and applicability is when we explicitly learn a state representation independently of the policy learning, and we train both models from scratches.

To what extent one can learn representations to learn better policies and policies to learn better representation?

What is the role of intrinsic rewards in this context?

\subsubsection{Unifying Model-free and Model-based Reinforcement Learning}

In this thesis, we chose to focus mainly on model-free algorithms and to rely upon simulators of the world to minimise the need for real-world interaction, which is

\footnotetext{
${ }^{5}$ Representations play a significant role also for exploring better and more efficiently.
} 
expensive, time consuming, and often infeasible. An exciting direction for future work $^{6}$ is one of combining model-free and model-based Reinforcement Learning to exploit the best of both worlds, i.e. high sample efficiency, intelligent exploration, and fast convergence. However, this road is hindered by the difficulties in learning valid models of the underlying MDPs, or POMDPs, in the samples collection approach and the generalisation of such models and policies to new tasks and environments. Advances in representation learning, meta-learning and intrinsic rewards play a crucial role in this framework.

To what extend one can unify model-fee and model-based Reinforcement Learning?

\subsubsection{Scaling to Real-world Problems}

Even in light of recent progress, complete Reinforcement Learning-based solutions to real-world robotics problems still seem far ahead into the future, due to the limited sample efficiency of the algorithms, and the consequent requirement of more and more data, the limited amount of real-world interaction, and the limited accuracy of virtual simulators. This issue is even more critical in the case of partial observability, which is common in real-world problems. Even if huge amounts of data are available, it is difficult to think that by only increasing the neural networks capacity and depth, we would be able to solve every problem.

Solutions proposing hybrid approaches ${ }^{7}$, combining learning-based with prior knowledge, often have better performances than pure learning-based approaches. This is especially important in robotics Deep Reinforcement Learning, where we want to limit the interaction with the real-world, and, consequently, we have limited numbers of samples available. However, the use of prior knowledge has to be dealt with carefully! The world is continuously changing and efficient agents have to be able to adapt to changes over their whole life time ${ }^{8}$. Prior knowledge cannot cover all the changes, can constrain solutions too much and limit their adaptability, and cannot solve problems we do not have yet solutions for. These are the reasons for using learning algorithms, but, when available, in combination

\footnotetext{
${ }^{6}$ This is a consequence of the studies done in Chapter 6 .

${ }^{7}$ For example, combining Reinforcement Learning and SLAM algorithm for mobile robot navigation and exploration problems.

${ }^{8}$ The branch of machine learning studying the problem of life-long learning is called continual learning.
} 
with loose prior knowledge ${ }^{9}$.

\subsection{Final Thoughts}

In this thesis, we investigated the problems of Reinforcement Learning in robotics and how prior knowledge can be incorporated and exploited in the different aspects of Reinforcement Learning, such as state representation, action representation, and shaping of reward functions. What we learned here can be used to make further progress in robotics Reinforcement Learning and, hopefully, to inspire and help researchers at the beginning of their journey in this exciting research field. Our thesis ends here, but our research, interests, and efforts in this field will not.

To conclude, we want to leave the reader with a final question for personal reflection on the future of intelligent and autonomous robotics:

Is not (reinforcement) learning the road to take?

\footnotetext{
${ }^{9}$ The concept of loose prior knowledge is discussed in Chapter 10.
} 



\section{Bibliography}

[1] Richard S. Sutton and Andrew G. Barto. Reinforcement learning: an introduction. Adaptive computation and machine learning series. The MIT Press, Cambridge, Massachusetts, second edition edition, 2018.

[2] S Russel and P Norvig. Artificial intelligence. A modern approach. Wiliams, 2007.

[3] P. H. Winston. Artificial Intelligence. Addison-Weasley, 1992.

[4] Richard Bellman. An introduction to artificial intelligence: Can computers think? Boyd and Fraser Publishing Company, 1978.

[5] Tom Ziemke. Embodied ai as science: Models of embodied cognition, embodied models of cognition, or both? In Embodied artificial intelligence, pages 27-36. Springer, 2004.

[6] Timothée Lesort, Natalia Díaz-Rodríguez, Jean-Franois Goudou, and David Filliat. State representation learning for control: An overview. Neural Networks, 108:379-392, 2018.

[7] Mohamed Abdellatif, Hazem Mohamed, M. Hesham, Ahmed Abdelmoneim, Amro Kamal, and Ahmed Khaled. Mechatronics design of an autonomous pipe-inspection robot. In MATEC Web Conf. Volume 153, 2018.

[8] A. Stentz. Optimal and efficient path planning for partially-known environments. International Conference on Robotics and Automation, pages 3310$3317,1994$.

[9] S. Thrun, W. Burgard, and D. Fox. Probabilistic robotics. MIT Press, 2005. 
[10] Jens Kober, J Andrew Bagnell, and Jan Peters. Reinforcement learning in robotics: A survey. The International Journal of Robotics Research, 32(11):1238-1274, 2013.

[11] Clive Dym. Principles of mathematical modeling. Elsevier, 2004.

[12] Nicolo Botteghi, Ruben Obbink, Daan Geijs, Mannes Poel, Beril Sirmacek, Christoph Brune, Abeje Mersha, and Stefano Stramigioli. Low dimensional state representation learning with reward-shaped priors. International Conference on Pattern Recognition, 2021.

[13] Nicolò Botteghi, Khaled Alaa, Mannes Poel, Beril Sirmacek, Christoph Brune, Abeje Mersha, and Stefano Stramigioli. Low dimensional state representation learning with robotics priors in continuous action spaces. International Conference on Intelligent Robots and Systems, 2021.

[14] Nicolò Botteghi, Mannes Poel, Beril Sirmacek, and Christoph Brune. Lowdimensional state and action representation learning with mdp homomorphism metrics. arXiv preprint arXiv:2107.0167\%, 2021.

[15] Nicolò Botteghi, Luuk Grefte, Mannes Poel, Beril Sirmacek, Christoph Brune, Edwin Dertien, and Stefano Stramigioli. Towards autonomous pipeline inspection with hierarchical reinforcement learning. arXiv preprint arXiv:210\%.03685, 2021.

[16] Khaled Alaa, Nicolò Botteghi, Beril Sirmacek, Mannes Poel, and Stefano Stramigioli. Towards continuous control for mobile robot navigation: A reinforcement learning and slam based approach. ISPRS - International Archives of the Photogrammetry, Remote Sensing and Spatial Information Sciences, XLII-2/W13:857-863, 2019.

[17] Nicolò Botteghi, Beril Sirmacek, Khaled Alaa, Mannes Poel, and Stefano Stramigioli. On reward shaping for mobile robot navigation: A reinforcement learning and slam based approach. arXiv preprint arXiv:2002.04109, 2020.

[18] Nicolo Botteghi, Khaled Alaa, Beril Sirmacek, and Mannes Poel. Entropybased exploration for mobile robot navigation: A learning-based approach. In Planning and Robotics Workshop, PlanRob, 2020. 
[19] N. Botteghi, B. Sirmacek, R. Schulte, M. Poel, and C. Brune. Reinforcement learning helps slam: Learning to build maps. ISPRS - International Archives of the Photogrammetry, Remote Sensing and Spatial Information Sciences, XLIII-B4-2020:329-335, 2020.

[20] Nicolò Botteghi, Rob Schulte, Beril Sirmacek, Mannes Poel, and Christoph Brune. Curiosity-Driven Reinforcement Learning Agent for Mapping Unknown Indoor Environments. ISPRS Annals of Photogrammetry, Remote Sensing and Spatial Information Sciences, 51:129-136, 2021.

[21] Richard Bellman. A markovian decision process. Journal of mathematics and mechanics, pages 679-684, 1957.

[22] A. Nowé, P. Vrancx, and Y-M. De Hauwere. Reinforcement Learning: Stateof-the-Art, chapter Game Theory and Multi-agent Reinforcement Learning, pages 441-470. Springer, 2012.

[23] Richard S Sutton, Andrew G Barto, et al. Introduction to reinforcement learning, volume 135. MIT press Cambridge, 1998.

[24] Kenji Doya. Temporal difference learning in continuous time and space. In Advances in neural information processing systems, pages 1073-1079, 1996.

[25] Dimitri P Bertsekas and John N Tsitsiklis. Neuro-dynamic programming. Athena Scientific, 1996.

[26] Ronald A Howard. Dynamic programming and markov processes. 1960.

[27] Martin L Puterman. Markov decision processes: discrete stochastic dynamic programming. John Wiley \& Sons, 2014.

[28] Satinder P Singh and Richard S Sutton. Reinforcement learning with replacing eligibility traces. Machine learning, 22(1-3):123-158, 1996.

[29] Christopher John Cornish Hellaby Watkins. Learning from delayed rewards. 1989.

[30] Richard S Sutton, David McAllester, Satinder Singh, and Yishay Mansour. Policy gradient methods for reinforcement learning with function approximation. Advances in neural information processing systems, 12:1057-1063, 1999. 
[31] Ronald J Williams. Simple statistical gradient-following algorithms for connectionist reinforcement learning. Machine learning, 8(3-4):229-256, 1992.

[32] David Silver, Guy Lever, Nicolas Heess, Thomas Degris, Daan Wierstra, and Martin Riedmiller. Deterministic policy gradient algorithms. In International conference on machine learning, pages 387-395. PMLR, 2014.

[33] Warren S McCulloch and Walter Pitts. A logical calculus of the ideas immanent in nervous activity. The bulletin of mathematical biophysics, 5(4):115133, 1943.

[34] Ian Goodfellow, Yoshua Bengio, Aaron Courville, and Yoshua Bengio. Deep learning, volume 1. MIT press Cambridge, 2016.

[35] Kurt Hornik, Maxwell Stinchcombe, Halbert White, et al. Multilayer feedforward networks are universal approximators. Neural networks, 2(5):359-366, 1989.

[36] Christopher M Bishop. Pattern recognition and machine learning. springer, 2006.

[37] Yann LeCun, Yoshua Bengio, et al. Convolutional networks for images, speech, and time series. The handbook of brain theory and neural networks, 3361(10):1995, 1995.

[38] David E Rumelhart, Geoffrey E Hinton, and Ronald J Williams. Learning representations by back-propagating errors. Nature, 323(6088):533-536, 1986.

[39] Alex Graves. Supervised sequence labelling. In Supervised sequence labelling with recurrent neural networks, pages 5-13. Springer, 2012.

[40] Volodymyr Mnih, Koray Kavukcuoglu, David Silver, Alex Graves, Ioannis Antonoglou, Daan Wierstra, and Martin Riedmiller. Playing atari with deep reinforcement learning. arXiv preprint arXiv:1312.5602, 2013.

[41] Volodymyr Mnih, Koray Kavukcuoglu, David Silver, Andrei A Rusu, Joel Veness, Marc G Bellemare, Alex Graves, Martin Riedmiller, Andreas K Fidjeland, Georg Ostrovski, et al. Human-level control through deep reinforcement learning. Nature, 518(7540):529-533, 2015. 
[42] Timothy P. Lillicrap, Jonathan J. Hunt, Alexander Pritzel, Nicolas Heess, Tom Erez, Yuval Tassa, David Silver, and Daan Wierstra. Continuous control with deep reinforcement learning. arXiv:1509.02971 [cs, stat], July 2019. arXiv: 1509.02971.

[43] George E Uhlenbeck and Leonard S Ornstein. On the theory of the brownian motion. Physical review, 36(5):823, 1930.

[44] Scott Fujimoto, Herke Hoof, and David Meger. Addressing function approximation error in actor-critic methods. In International Conference on Machine Learning, pages 1587-1596. PMLR, 2018.

[45] Hado Van Hasselt, Arthur Guez, and David Silver. Deep reinforcement learning with double q-learning. In Proceedings of the AAAI conference on artificial intelligence, volume 30, 2016.

[46] John Schulman, Filip Wolski, Prafulla Dhariwal, Alec Radford, and Oleg Klimov. Proximal policy optimization algorithms. arXiv preprint arXiv:1707.06347, 2017.

[47] John Schulman, Sergey Levine, Pieter Abbeel, Michael Jordan, and Philipp Moritz. Trust region policy optimization. In International conference on machine learning, pages 1889-1897, 2015.

[48] David Silver, Aja Huang, Chris J Maddison, Arthur Guez, Laurent Sifre, George Van Den Driessche, Julian Schrittwieser, Ioannis Antonoglou, Veda Panneershelvam, Marc Lanctot, et al. Mastering the game of go with deep neural networks and tree search. Nature, 529(7587):484-489, 2016.

[49] David Silver, Julian Schrittwieser, Karen Simonyan, Ioannis Antonoglou, Aja Huang, Arthur Guez, Thomas Hubert, Lucas Baker, Matthew Lai, Adrian Bolton, et al. Mastering the game of go without human knowledge. Nature, 550(7676):354-359, 2017.

[50] Manuel Watter, Jost Tobias Springenberg, Joschka Boedecker, and Martin Riedmiller. Embed to control: A locally linear latent dynamics model for control from raw images. arXiv preprint arXiv:1506.07365, 2015. 
[51] Sergey Levine, Chelsea Finn, Trevor Darrell, and Pieter Abbeel. End-toend training of deep visuomotor policies. The Journal of Machine Learning Research, 17(1):1334-1373, 2016.

[52] Wendelin Böhmer, Jost Tobias Springenberg, Joschka Boedecker, Martin Riedmiller, and Klaus Obermayer. Autonomous learning of state representations for control: An emerging field aims to autonomously learn state representations for reinforcement learning agents from their real-world sensor observations. KI-Künstliche Intelligenz, 29(4):353-362, 2015.

[53] Yann Le Cun and Françoise Fogelman-Soulié. Modèles connexionnistes de l'apprentissage. Intellectica, 2(1):114-143, 1987.

[54] Chelsea Finn, Xin Yu Tan, Yan Duan, Trevor Darrell, Sergey Levine, and Pieter Abbeel. Deep spatial autoencoders for visuomotor learning. In 2016 IEEE International Conference on Robotics and Automation (ICRA), pages 512-519. IEEE, 2016.

[55] Jan Mattner, Sascha Lange, and Martin Riedmiller. Learn to swing up and balance a real pole based on raw visual input data. In Tingwen Huang, Zhigang Zeng, Chuandong Li, and Chi Sing Leung, editors, Neural Information Processing, pages 126-133, Berlin, Heidelberg, 2012. Springer Berlin Heidelberg.

[56] Samuel Alvernaz and Julian Togelius. Autoencoder-augmented neuroevolution for visual doom playing. IEEE Conference on Computational Intelligence and Games, Aug 2017.

[57] Diederik P Kingma and Max Welling. Stochastic gradient vb and the variational auto-encoder. In International Conference on Learning Representations, volume 19, 2014.

[58] Yoshua Bengio, Li Yao, Guillaume Alain, and Pascal Vincent. Generalized denoising auto-encoders as generative models. Advances in neural information processing systems, 26:899-907, 2013.

[59] Ross Goroshin, Michael Mathieu, and Yann LeCun. Learning to linearize under uncertainty, 2015. 
[60] Herke Van Hoof, Nutan Chen, Maximilian Karl, Patrick van der Smagt, and Jan Peters. Stable reinforcement learning with autoencoders for tactile and visual data. In 2016 IEEE/RSJ international conference on intelligent robots and systems, pages 3928-3934. IEEE, 2016.

[61] Pulkit Agrawal, Ashvin Nair, Pieter Abbeel, Jitendra Malik, and Sergey Levine. Learning to poke by poking: Experiential learning of intuitive physics. arXiv preprint arXiv:1606.07419, 2016.

[62] Carles Gelada, Saurabh Kumar, Jacob Buckman, Ofir Nachum, and Marc G Bellemare. Deepmdp: Learning continuous latent space models for representation learning. In International Conference on Machine Learning, pages 2170-2179. PMLR, 2019.

[63] Rico Jonschkowski and Oliver Brock. Learning state representations with robotic priors. Autonomous Robots, 39(3):407-428, 2015.

[64] Rico Jonschkowski, Roland Hafner, Jonathan Scholz, and Martin Riedmiller. Pves: Position-velocity encoders for unsupervised learning of structured state representations. arXiv preprint arXiv:1705.09805, 2017.

[65] Timothée Lesort, Mathieu Seurin, Xinrui Li, Natalia Díaz-Rodríguez, and David Filliat. Unsupervised state representation learning with robotic priors: a robustness benchmark. arXiv preprint arXiv:1709.05185, 2017.

[66] Marco Morik, Divyam Rastogi, Rico Jonschkowski, and Oliver Brock. State representation learning with robotic priors for partially observable environments. In 2019 IEEE/RSJ International Conference on Intelligent Robots and Systems (IROS), pages 6693-6699. IEEE, 2019.

[67] Richard S Sutton, Doina Precup, and Satinder Singh. Between mdps and semi-mdps: A framework for temporal abstraction in reinforcement learning. Artificial intelligence, 112(1-2):181-211, 1999.

[68] Christopher Atkeson. Using local trajectory optimizers to speed up global optimization in dynamic programming. Advances in neural information processing systems, 6:663-670, 1993.

[69] Andrew Y Ng, Daishi Harada, and Stuart Russell. Policy invariance under reward transformations: Theory and application to reward shaping. In 
International Conference on Machine Learning, volume 99, pages 278-287, 1999.

[70] Adam Daniel Laud. Theory and application of reward shaping in reinforcement learning. Technical report, 2004.

[71] Stuart Russell. Learning agents for uncertain environments. In Proceedings of the eleventh annual conference on Computational learning theory, pages 101-103, 1998.

[72] Jens Kober and Jan Peters. Imitation and reinforcement learning. IEEE Robotics $\&$ Automation Magazine, 17(2):55-62, 2010.

[73] Pieter Abbeel and Andrew Y Ng. Apprenticeship learning via inverse reinforcement learning. In Proceedings of the twenty-first international conference on Machine learning, page 1, 2004.

[74] Andrew G Barto and Sridhar Mahadevan. Recent advances in hierarchical reinforcement learning. Discrete event dynamic systems, 13(1-2):41-77, 2003.

[75] Deepak Pathak, Pulkit Agrawal, Alexei A Efros, and Trevor Darrell. Curiosity-driven exploration by self-supervised prediction. In International conference on machine learning, pages 2778-2787. PMLR, 2017.

[76] Nikolay Savinov, Anton Raichuk, Raphaël Marinier, Damien Vincent, Marc Pollefeys, Timothy Lillicrap, and Sylvain Gelly. Episodic curiosity through reachability. arXiv preprint arXiv:1810.02274, 2018.

[77] Yoshua Bengio, Aaron Courville, and Pascal Vincent. Representation learning: A review and new perspectives. IEEE transactions on pattern analysis and machine intelligence, 35(8):1798-1828, 2013.

[78] Tim De Bruin, Jens Kober, Karl Tuyls, and Robert Babuška. Integrating state representation learning into deep reinforcement learning. IEEE Robotics and Automation Letters, 3(3):1394-1401, 2018.

[79] Svante Wold, Kim Esbensen, and Paul Geladi. Principal component analysis. Chemometrics and intelligent laboratory systems, 2(1-3):37-52, 1987.

[80] Laurens Van der Maaten and Geoffrey Hinton. Visualizing data using t-sne. Journal of machine learning research, 9(11), 2008. 
[81] Petar Kormushev, Sylvain Calinon, and Darwin G Caldwell. Reinforcement learning in robotics: Applications and real-world challenges. Robotics, 2(3):122-148, 2013.

[82] Timothée Lesort, Mathieu Seurin, Xinrui Li, Natalia Díaz-Rodríguez, and David Filliat. Unsupervised state representation learning with robotic priors: a robustness benchmark. arXiv preprint arXiv:1709.05185, 2017.

[83] Marco Morik, Divyam Rastogi, Rico Jonschkowski, and Oliver Brock. State representation learning with robotic priors for partially observable environments. In International Conference on Intelligent Robots and Systems, pages 6693-6699, 2019.

[84] L. Tai, G. Paolo, and M. Liu. Virtual-to-real deep reinforcement learning: Continuous control of mobile robots for mapless navigation. International Conference on Intelligent Robots and Systems, pages 31-36, 2017.

[85] Antonin Raffin, Ashley Hill, René Traoré, Timothée Lesort, Natalia DíazRodríguez, and David Filliat. Decoupling feature extraction from policy learning: assessing benefits of state representation learning in goal based robotics. arXiv preprint arXiv:1901.08651, 2019.

[86] Vincent François-Lavet, Yoshua Bengio, Doina Precup, and Joelle Pineau. Combined reinforcement learning via abstract representations. In Proceedings of the AAAI Conference on Artificial Intelligence, volume 33, pages 3582-3589, 2019.

[87] Elise van der Pol, Thomas Kipf, Frans A Oliehoek, and Max Welling. Plannable approximations to mdp homomorphisms: Equivariance under actions. arXiv preprint arXiv:2002.11963, 2020.

[88] Gabriel Dulac-Arnold, Richard Evans, Hado van Hasselt, Peter Sunehag, Timothy Lillicrap, Jonathan Hunt, Timothy Mann, Theophane Weber, Thomas Degris, and Ben Coppin. Deep reinforcement learning in large discrete action spaces. arXiv preprint arXiv:1512.07679, 2015.

[89] Dylan P Losey, Krishnan Srinivasan, Ajay Mandlekar, Animesh Garg, and Dorsa Sadigh. Controlling assistive robots with learned latent actions. In 
2020 IEEE International Conference on Robotics and Automation (ICRA), pages 378-384. IEEE, 2020.

[90] Yash Chandak, Georgios Theocharous, James Kostas, Scott Jordan, and Philip Thomas. Learning action representations for reinforcement learning. In International Conference on Machine Learning, pages 941-950. PMLR, 2019.

[91] Balaraman Ravindran and Andrew G Barto. Symmetries and model minimization in markov decision processes, 2001.

[92] Balaraman Ravindran and Andrew G Barto. Approximate homomorphisms: A framework for non-exact minimization in markov decision processes. 2004.

[93] Jonathan Taylor, Doina Precup, and Prakash Panagaden. Bounding performance loss in approximate mdp homomorphisms. Advances in Neural Information Processing Systems, 21:1649-1656, 2008.

[94] Chelsea Finn, Xin Yu Tan, Yan Duan, Trevor Darrell, Sergey Levine, and Pieter Abbeel. Deep spatial autoencoders for visuomotor learning. In 2016 IEEE International Conference on Robotics and Automation (ICRA), pages 512-519. IEEE, 2016.

[95] Jan Mattner, Sascha Lange, and Martin Riedmiller. Learn to swing up and balance a real pole based on raw visual input data. In International Conference on Neural Information Processing, pages 126-133. Springer, 2012.

[96] Herke Van Hoof, Nutan Chen, Maximilian Karl, Patrick van der Smagt, and Jan Peters. Stable reinforcement learning with autoencoders for tactile and visual data. In 2016 IEEE/RSJ international conference on intelligent robots and systems (IROS), pages 3928-3934. IEEE, 2016.

[97] Niklas Wahlström, Thomas B Schön, and Marc Peter Deisenroth. From pixels to torques: Policy learning with deep dynamical models. arXiv preprint arXiv:1502.02251, 2015.

[98] Martin Stolle and Doina Precup. Learning options in reinforcement learning. In International Symposium on abstraction, reformulation, and approximation, pages 212-223. Springer, 2002. 
[99] Thomas G Dietterich. The maxq method for hierarchical reinforcement learning. In ICML, volume 98, pages 118-126. Citeseer, 1998.

[100] Alexander Sasha Vezhnevets, Simon Osindero, Tom Schaul, Nicolas Heess, Max Jaderberg, David Silver, and Koray Kavukcuoglu. Feudal networks for hierarchical reinforcement learning. arXiv preprint arXiv:1703.01161, 2017.

[101] Paul J Pritz, Liang Ma, and Kin K Leung. Joint state-action embedding for efficient reinforcement learning. arXiv preprint arXiv:2010.04444, 2020.

[102] Thomas Kipf, Elise van der Pol, and Max Welling. Contrastive learning of structured world models. arXiv preprint arXiv:1911.1224\%, 2019.

[103] Maxime Chevalier-Boisvert, Lucas Willems, and Suman Pal. Minimalistic gridworld environment for openai gym. https://github.com/maximecb/ gym-minigrid, 2018.

[104] Eric Rohmer, Surya PN Singh, and Marc Freese. V-rep: A versatile and scalable robot simulation framework. In 2013 IEEE/RSJ International Conference on Intelligent Robots and Systems, pages 1321-1326. IEEE, 2013.

[105] Stephen James, Marc Freese, and Andrew J Davison. Pyrep: Bringing v-rep to deep robot learning. arXiv preprint arXiv:1906.11176, 2019.

[106] Max Jaderberg, Volodymyr Mnih, Wojciech Marian Czarnecki, Tom Schaul, Joel Z Leibo, David Silver, and Koray Kavukcuoglu. Reinforcement learning with unsupervised auxiliary tasks. arXiv preprint arXiv:1611.05397, 2016.

[107] Alireza Makhzani, Jonathon Shlens, Navdeep Jaitly, Ian Goodfellow, and Brendan Frey. Adversarial autoencoders. arXiv preprint arXiv:1511.05644, 2015.

[108] Ashish Vaswani, Noam Shazeer, Niki Parmar, Jakob Uszkoreit, Llion Jones, Aidan N Gomez, Lukasz Kaiser, and Illia Polosukhin. Attention is all you need. arXiv preprint arXiv:1706.03762, 2017.

[109] Ofir Nachum, Shixiang Shane Gu, Honglak Lee, and Sergey Levine. Dataefficient hierarchical reinforcement learning. In Advances in Neural Information Processing Systems, pages 3303-3313, 2018. 
[110] Nicolas Heess, Greg Wayne, Yuval Tassa, Timothy Lillicrap, Martin Riedmiller, and David Silver. Learning and transfer of modulated locomotor controllers. arXiv preprint arXiv:1610.05182, 2016.

[111] Carlos Florensa, Yan Duan, and Pieter Abbeel. Stochastic neural networks for hierarchical reinforcement learning. arXiv preprint arXiv:1704.03012, 2017.

[112] Kevin Frans, Jonathan Ho, Xi Chen, Pieter Abbeel, and John Schulman. Meta learning shared hierarchies. arXiv preprint arXiv:1710.09767, 2017.

[113] Pierre-Luc Bacon, Jean Harb, and Doina Precup. The option-critic architecture. In Proceedings of the AAAI Conference on Artificial Intelligence, volume 31, 2017.

[114] Edwin Dertien, Stefano Stramigioli, and Kees Pulles. Development of an inspection robot for small diameter gas distribution mains. In 2011 IEEE International Conference on Robotics and Automation, pages 5044-5049. IEEE, 2011.

[115] G.H. Mills, A.E. Jackson, and R.C Richardson. Advances in the inspection of unpiggable pipelines. Robotics 6, 2017.

[116] Erich Rome, Joachim Hertzberg, Frank Kirchner, Ulrich Licht, and Thomas Christaller. Towards autonomous sewer robots: the MAKRO project. Urban Water, 1(1):57-70, March 1999.

[117] Hyouk Ryeol Choi and S. M. Ryew. Robotic system with active steering capability for internal inspection of urban gas pipelines. In Mechatronics Volume 12, 2002.

[118] Anojan Selvarajan, Anirban Kumar, Devika Sethu, and Mohd Azwan bin Ramlan. Design and development of a snake-robot for pipeline inspection. 2019 IEEE Student Conference on Research and Development (SCOReD), pages 237-242, 2019.

[119] Paulo Debenest, Michele Guarnieri, and Shigeo Hirose. Pipetron series robots for pipe inspection. Proceedings of the $20143 \mathrm{rd}$ International Conference on Applied Robotics for the Power Industry, pages 1-6, 2014. 
[120] Amir Ali Forough Nassiraei, Yoshinori Kawamura, Alireza Ahrary, Yoshikazu Mikuriya, and Kazumasa Ishii. Concept and design of a fully autonomous sewer pipe inspection mobile robot "kantaro". Proceedings 2007 IEEE International Conference on Robotics and Automation, pages 136-143, 2007.

[121] Sebastian Thrun. Probabilistic robotics. Communications of the ACM, $45(3): 52-57,2002$.

[122] E. Rohmer, S. P. N. Singh, and M. Freese. Coppeliasim (formerly v-rep): a versatile and scalable robot simulation framework. In Proc. of The International Conference on Intelligent Robots and Systems (IROS), 2013. www.coppeliarobotics.com.

[123] Stephen James, Andrew J Davison, and Edward Johns. Transferring end-toend visuomotor control from simulation to real world for a multi-stage task. In Conference on Robot Learning, pages 334-343. PMLR, 2017.

[124] Zackory Erickson, Vamsee Gangaram, Ariel Kapusta, C Karen Liu, and Charles C Kemp. Assistive gym: A physics simulation framework for assistive robotics. In 2020 IEEE International Conference on Robotics and Automation (ICRA), pages 10169-10176. IEEE, 2020.

[125] Philipp Moritz, Robert Nishihara, Stephanie Wang, Alexey Tumanov, Richard Liaw, Eric Liang, Melih Elibol, Zongheng Yang, William Paul, Michael I. Jordan, and Ion Stoica. Ray: A Distributed Framework for Emerging AI Applications. arXiv:1712.05889 [cs, stat], September 2018. arXiv: 1712.05889 .

[126] Greg Brockman, Vicki Cheung, Ludwig Pettersson, Jonas Schneider, John Schulman, Jie Tang, and Wojciech Zaremba. Openai gym, 2016.

[127] Jan Matas, Stephen James, and Andrew J Davison. Sim-to-real reinforcement learning for deformable object manipulation. In Conference on Robot Learning, pages 734-743. PMLR, 2018.

[128] Oleksii Zhelo, Jingwei Zhang, Lei Tai, Ming Liu, and Wolfram Burgard. Curiosity-driven exploration for mapless navigation with deep reinforcement learning. arXiv preprint arXiv:1804.00456, 2018. 
[129] Mark Pfeiffer, Michael Schaeuble, Juan Nieto, Roland Siegwart, and Cesar Cadena. From perception to decision: A data-driven approach to end-to-end motion planning for autonomous ground robots. 2017 IEEE International Conference on Robotics and Automation (ICRA), May 2017.

[130] N. Duo, Q. Wang, Q. Lv, H. Wei, and P. Zhang. A deep reinforcement learning based mapless navigation algorithm using continuous actions. In 2019 International Conference on Robots Intelligent System (ICRIS), pages 63-68, June 2019.

[131] Wei Zhang, Yunfeng Zhang, and Ning Liu. Danger-aware adaptive composition of drl agents for self-navigation. Unmanned Systems, 9(01):1-9, 2021.

[132] J. Zhang, J. Springenberg, J. Boedecker, and W. Burgard. Deep reinforcement learning with successor features for navigation across similar environments. arXiv:161205533, 2016.

[133] J. Zhang, L. Tai, J. Boedecker, and M. Liu. Neural slam: Learning to explore with external memory. arXiv:170609520, 2017.

[134] G. Brunner, O. Richter, Y. Wang, and R. Wattenhofer. Teaching a machine to read maps with deep reinforcement learning. arXiv:171107479, 2017.

[135] Randall Smith, Matthew Self, and Peter Cheeseman. Estimating Uncertain Spatial Relationships in Robotics, pages 167-193. Springer New York, New York, NY, 1990.

[136] Anthony Stentz, Dieter Fox, and Michael Montemerlo. Fastslam: A factored solution to the simultaneous localization and mapping problem with unknown data association. In In proceedings of the AAAI national conference on artificial intelligence. Citeseer, 2003.

[137] Raul Mur-Artal, J. Montiel, and Juan Tardos. Orb-slam: a versatile and accurate monocular slam system. IEEE Transactions on Robotics, 31:1147 - 1163, 102015.

[138] Takafumi Taketomi, Hideaki Uchiyama, and Sei Ikeda. Visual slam algorithms: a survey from 2010 to 2016. IPSJ Transactions on Computer Vision and Applications, 9(1):16, 2017. 
[139] Giorgio Grisettiyz, Cyrill Stachniss, and Wolfram Burgard. Improving gridbased slam with rao-blackwellized particle filters by adaptive proposals and selective resampling. In Proceedings of the 2005 IEEE international conference on robotics and automation, pages 2432-2437. IEEE, 2005.

[140] K. Murphy. Bayesian map learning in dynamic environments. Neural Information Processing Systems, 12:1015-1021, 1999.

[141] H. Moravec. Sensor fusion in certainty grids for mobile robots. AI Magazine, pages $61-74,1988$.

[142] Diederik P Kingma and Jimmy Ba. Adam: A method for stochastic optimization. arXiv preprint arXiv:1412.6980, 2014.

[143] Timothy P Lillicrap, Jonathan J Hunt, Alexander Pritzel, Nicolas Heess, Tom Erez, Yuval Tassa, David Silver, and Daan Wierstra. Continous control with deep reinforcement learning. International Conference on Learning Representations, 2016.

[144] Giorgio Grisetti, Cyrill Stachniss, and Wolfram Burgard. Improved techniques for grid mapping with rao-blackwellized particle filters. Robotics, IEEE Transactions on, 23:34 - 46, 032007.

[145] G. Grisetti, C. Stachniss, and W. Burgard. Improving grid-based slam with rao-blackwellized particle filters by adaptive proposals and selective resampling. Proceedings of the 2005 IEEE international conference on robotics and automation., 2005.

[146] D. Fox, W. Burgard, and S. Thrun. The dynamic window approach to collision avoidance. Robotics and Automation Magazine, IEEE, 4:23-33, 1997.

[147] B. Yamauchi. A frontier-based approach for autonomous exploration. In Proceedings 1997 IEEE International Symposium on Computational Intelligence in Robotics and Automation CIRA'97. 'Towards New Computational Principles for Robotics and Automation', pages 146-151, 1997.

[148] Deepak Pathak, Pulkit Agrawal, Alexei A Efros, and Trevor Darrell. Curiosity-driven exploration by self-supervised prediction. In International conference on machine learning, pages 2778-2787. PMLR, 2017. 
[149] Thomas Kollar and Nicholas Roy. Trajectory optimization using reinforcement learning for map exploration. The International Journal of Robotics Research, 27(2):175-196, 2008.

[150] Tao Chen, Saurabh Gupta, and Abhinav Gupta. Learning exploration policies for navigation. International Conference on Learning Representations, 2019.

[151] Ramezani Dooraki, Amir, Lee, and Deok-Jin. An end-to-end deep reinforcement learning-based intelligent agent capable of autonomous exploration in unknown environments. Sensors, 18(10):3575, 2018.

[152] Julio A Placed and José A Castellanos. A deep reinforcement learning approach for active slam. Applied Sciences, 10(23):8386, 2020.

[153] Farzad Niroui, Kaicheng Zhang, Zendai Kashino, and Goldie Nejat. Deep reinforcement learning robot for search and rescue applications: Exploration in unknown cluttered environments. IEEE Robotics and Automation Letters, $4(2): 610-617,2019$.

[154] Brian Yamauchi. A frontier-based approach for autonomous exploration. Proceedings 1997 IEEE International Symposium on Computational Intelligence in Robotics and Automation CIRA'97. 'Towards New Computational Principles for Robotics and Automation', pages 146-151, 1997.

[155] Fabio Pardo, Arash Tavakoli, Vitaly Levdik, and Petar Kormushev. Time limits in reinforcement learning. In International Conference on Machine Learning, pages 4045-4054. PMLR, 2018.

[156] Yuri Burda, Harri Edwards, Deepak Pathak, Amos Storkey, Trevor Darrell, and Alexei A Efros. Large-scale study of curiosity-driven learning. arXiv preprint arXiv:1808.04355, 2018.

[157] funda.nl. https://www.funda.nl/. 2020. Houses in the Netherlands, [Online; accessed April 2020]. 
Appendix A

Proof of Proposition 2 


\section{Equivalence Between Intermediate Policy and La- tent Policy}

Given the relation between the intermediate policy $\pi_{i}$ and the latent policy $\bar{\pi}^{1}$ :

$$
\pi_{i}(a \mid \bar{s}):=\int_{\delta_{d}^{-1}(a)} \bar{\pi}(\bar{a} \mid \bar{s}) \mathrm{d} \bar{a}
$$

We can express the performance measure of the intermediate policy with as:

$$
\begin{aligned}
J_{\pi_{i}}\left(\boldsymbol{\theta}_{\bar{\pi}}, \boldsymbol{\theta}_{\delta_{d}}\right) & =\sum_{\bar{s} \in \overline{\mathcal{S}}} d_{0}(\bar{s}) \mathrm{V}^{\pi_{i}}(\bar{s}) \\
& =\sum_{\bar{s} \in \overline{\mathcal{S}}} d_{0}(\bar{s}) \sum_{a \in \mathcal{A}} \int_{\delta_{d}^{-1}(a)} \bar{\pi}(\bar{a} \mid \bar{s}) \mathrm{Q}^{\pi_{i}}(\bar{s}, a) \mathrm{d} \bar{a}
\end{aligned}
$$

If we now take the gradient of the performance measure of the intermediate policy, we obtain:

$$
\nabla_{\boldsymbol{\theta}_{\bar{\pi}}} J_{\pi_{i}}\left(\boldsymbol{\theta}_{\bar{\pi}}, \boldsymbol{\theta}_{\delta_{d}}\right)=\nabla_{\boldsymbol{\theta}_{\bar{\pi}}}\left[\sum_{\bar{s} \in \overline{\mathcal{S}}} d_{0}(\bar{s}) \sum_{a \in \mathcal{A}} \int_{\delta_{d}^{-1}(a)} \bar{\pi}(\bar{a} \mid \bar{s}) \mathrm{Q}^{\pi_{i}}(\bar{s}, a) \mathrm{d} e\right]
$$

Using the policy gradient theorem [1] for the intermediate policy $\pi_{i}$, we can rewrite Equation (A.3) as:

$$
\begin{aligned}
\nabla_{\boldsymbol{\theta}_{\bar{\pi}}} J_{\pi_{i}}\left(\boldsymbol{\theta}_{\bar{\pi}}, \boldsymbol{\theta}_{\delta_{d}}\right) & =\sum_{t=0}^{\infty} \mathbb{E}\left[\sum_{a \in \mathcal{A}} \gamma^{t} \mathrm{Q}^{\pi_{i}}\left(\bar{S}_{t}, a\right) \nabla_{\boldsymbol{\theta}_{\bar{\pi}}}\left(\int_{\delta_{d}^{-1}(a)} \bar{\pi}\left(\bar{a} \mid \bar{S}_{t}\right) \mathrm{d} \bar{a}\right)\right] \\
& =\sum_{t=0}^{\infty} \mathbb{E}\left[\sum_{a \in \mathcal{A}} \gamma^{t} \int_{\delta_{d}^{-1}(a)} \nabla_{\boldsymbol{\theta}_{\bar{\pi}}}\left(\bar{\pi}\left(\bar{a} \mid \bar{S}_{t}\right)\right) \mathrm{Q}^{\pi_{i}}\left(\bar{S}_{t}, a\right) \mathrm{d} \bar{a}\right] \\
& =\sum_{t=0}^{\infty} \mathbb{E}\left[\sum_{a \in \mathcal{A}} \gamma^{t} \int_{\delta_{d}^{-1}(a)} \bar{\pi}\left(\bar{a} \mid \bar{S}_{t}\right) \nabla_{\boldsymbol{\theta}_{\bar{\pi}}} \ln \left(\bar{\pi}\left(\bar{a} \mid \bar{S}_{t}\right)\right) \mathrm{Q}^{\pi_{i}}\left(\bar{S}_{t}, a\right) \mathrm{d} \bar{a}\right]
\end{aligned}
$$

Because latent actions are deterministically mapped to actions, $\mathrm{Q}^{\pi_{i}}\left(\bar{S}_{t}, a\right)=$

\footnotetext{
${ }^{1}$ The proof is adapted from [90]
} 
$\mathrm{Q}^{\bar{\pi}}\left(\bar{S}_{t}, \bar{a}\right)$. Thus:

$\nabla_{\boldsymbol{\theta}_{\bar{\pi}}} J_{\pi_{i}}\left(\boldsymbol{\theta}_{\bar{\pi}}, \boldsymbol{\theta}_{\delta_{d}}\right)=\sum_{t=0}^{\infty} \mathbb{E}\left[\gamma^{t} \sum_{a \in \mathcal{A}} \int_{\delta_{d}^{-1}(a)} \bar{\pi}\left(\bar{a} \mid \bar{S}_{t}\right) \nabla_{\boldsymbol{\theta}_{\bar{\pi}}} \ln \left(\bar{\pi}\left(\bar{a} \mid \bar{S}_{t}\right)\right) \mathrm{Q}^{\pi_{i}}\left(\bar{S}_{t}, \bar{a}\right) \mathrm{d} \bar{a}\right]$

Eventually, the summation over $a$ and the integral over $\delta_{d}(a)$ can by replace by the integral over the domain of the latent action space $\bar{a}$. Therefore:

$$
\begin{aligned}
\nabla_{\boldsymbol{\theta}_{\bar{\pi}}} J_{\pi_{i}}\left(\boldsymbol{\theta}_{\bar{\pi}}, \boldsymbol{\theta}_{\delta_{d}}\right) & =\sum_{t=0}^{\infty} \mathbb{E}\left[\gamma^{t} \int_{\bar{a}} \bar{\pi}\left(\bar{a} \mid \bar{S}_{t}\right) \nabla_{\boldsymbol{\theta}_{\bar{\pi}}} \ln \left(\bar{\pi}\left(\bar{a} \mid \bar{S}_{t}\right)\right) \mathrm{Q}^{\bar{\pi}}\left(\bar{S}_{t}, \bar{a}\right) \mathrm{d} \bar{a}\right] \\
& =\sum_{t=0}^{\infty} \mathbb{E}\left[\gamma^{t} \int_{\bar{a}} \mathrm{Q}^{\bar{\pi}}\left(\bar{S}_{t}, \bar{a}\right) \nabla_{\boldsymbol{\theta}_{\bar{\pi}}} \bar{\pi}\left(\bar{a} \mid \bar{S}_{t}\right) \mathrm{d} \bar{a}\right] \\
& =\nabla_{\boldsymbol{\theta}_{\bar{\pi}}} J_{\bar{\pi}}\left(\boldsymbol{\theta}_{\bar{\pi}}\right)
\end{aligned}
$$



Appendix B

Supplementary Results 


\section{State and Action Representation Learned in a Grid- world}

The learned action representation and the latent transitions in the $6 \times 6$ maze are shown in Figure B.1.

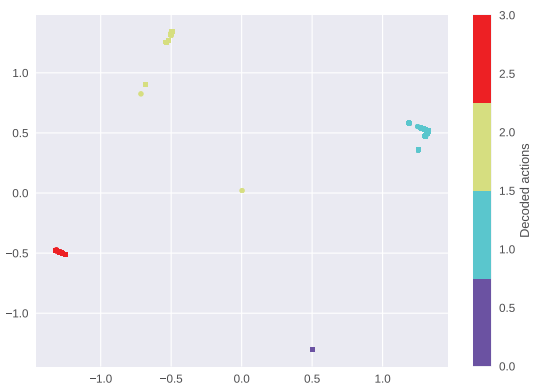

(a) Maze $6 \times 6$, latent actions

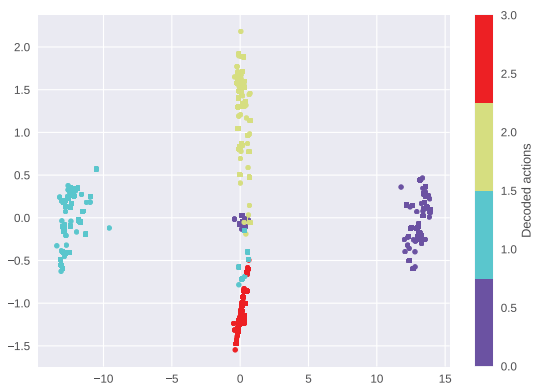

(b) Maze $6 \times 6$, latent transitions

Figure B.1: First two principal components of the learned action space $\overline{\mathcal{A}}$ and the $\Delta \overline{\mathrm{T}}$ in the $6 \times 6$ grid-worlds in Figure 6.8a.

The learned representations in the $6 \times 6$ grid-world are shown in Figure B.2. 


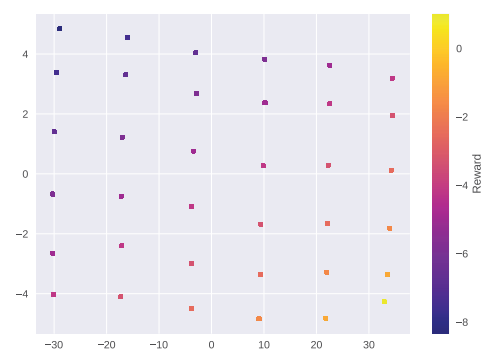

(a) Our

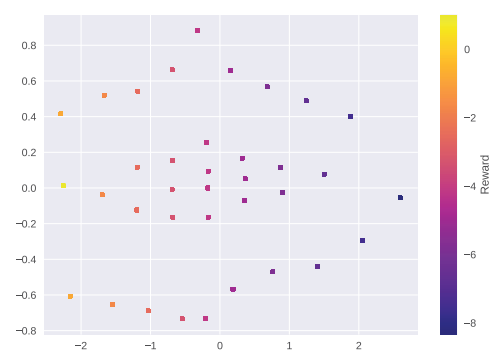

(c) D-MDP $[62]$

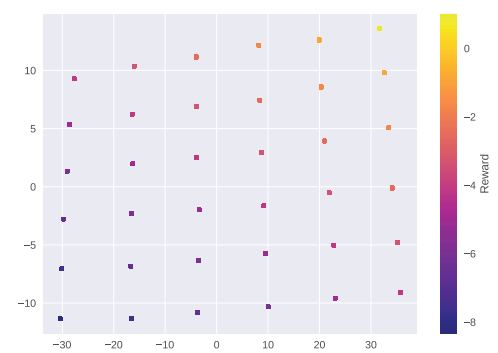

(b) MDP-H [87]

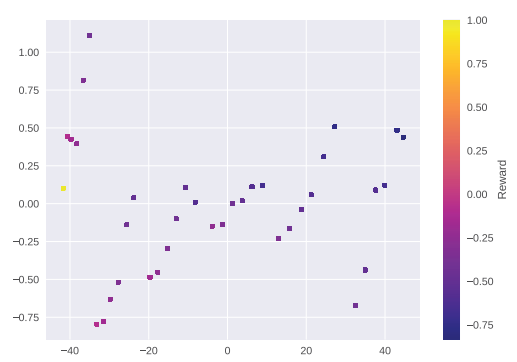

(d) JSAE [101]

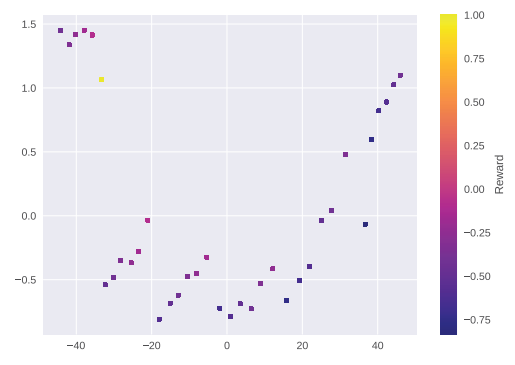

(e) JSAE-C [101]

Figure B.2: First two principal components of the learned state representations of the $6 \times 6$ grid-world in Figure 6.8 a. The color of the state predictions is done accordingly to the reward function in Equation (6.13). 



\section{Biography}

Nicolò Botteghi was born on November 10, 1992, in Bologna, Italy. He studied at Liceo Scientifico Malpighi and obtained his high-school diploma in 2011. He has always liked studying and learning, especially scientific disciplines, such as mathematics, chemistry, and physics.

He obtained his Bachelor and Master degree (cum laude) in Automation Engineering at Alma Mater Studiorum - Università di Bologna in 2014 and 2017, respectively. During his studies, he explored and expanded his knowledge in many different engineering fields, such as robotics, control, electronics, mechanics and computer science. He carried out his Master thesis at the University of Twente, where he developed a controller for a four-legged cheetah robot.

During his PhD, he focused on Artificial Intelligence and Deep Reinforcement Learning for autonomous robotics. With great effort and passion, he vastly increased his knowledge of different aspects of science. He also taught different courses and supervised many students during their final projects.

Nicolò has always been an athlete since the age of five when he started playing basketball. He received several awards and played professional basketball for two years after high school. Since 2017, he has been an active member of the Aloha Triathlon Team of the University of Twente, and in 2019 he completed his first IRONMAN 70.3.
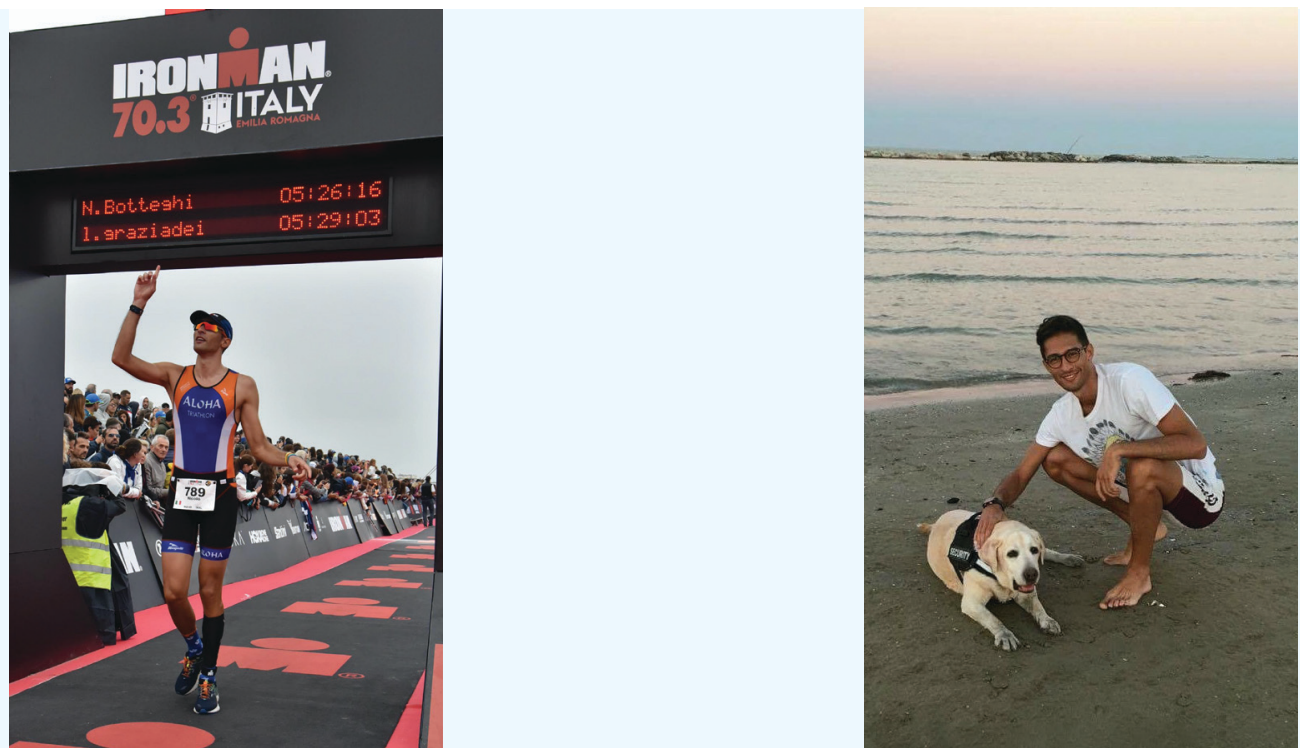
